\title{
49th ESCP virtual symposium on clinical pharmacy 19.10.2021-21.10.2021 Clinical pharmacy, working collaboratively in mental health care
}

Published online: 29 November 2021

(C) Springer Nature Switzerland AG 2021

\section{Oral communications and Posters}

\section{ORAL COMMUNICATION I}

\section{OR01.1}

Developing and validating a prediction model for in-hospital mortality associated with anticholinergic drug burden among older hospitalised patients with dementia

Angela E. Lisibach ${ }^{1,2,3,4}$, Nora Haltinner ${ }^{*}$, 5 , Patrick E. Beeler ${ }^{6}$ Andrea Burden ${ }^{5}$, Chantal Csajka ${ }^{1,2,3}$, Monika Lutters ${ }^{4,5}$

${ }^{1}$ Institute of Pharmaceutical Sciences of Western Switzerland, University of Geneva, University of Lausanne, ${ }^{2} \mathrm{School}$ of Pharmaceutical Sciences, University of Geneva, Geneva, ${ }^{3}$ Center for Research and Innovation in Clinical Pharmaceutical Sciences, University Hospital and University of Lausanne, Lausanne, ${ }^{4}$ Departement of Medical Services, Clinical Pharmacy, Cantonal Hospital of Baden, Baden, ${ }^{5}$ Department of Chemistry and Applied Biosciences, Institute of Pharmaceutical Sciences, ETH Zurich, Zurich, ${ }^{6}$ Division of Occupational and Environmental Medicine, Epidemiology, Biostatistics and Prevention Institute, University of Zurich and University Hospital Zurich, Zürich, Switzerland

Background and Objective: After cardiovascular diseases and cancer, dementia is the third most frequent cause of death in Switzerland among patients aged 65 years and older. A recent cohort study conducted in a regional hospital in Switzerland showed that a high anticholinergic drug burden in older patients is significantly associated with increased in-hospital mortality. We aimed to develop and validate a risk prediction model for in-hospital mortality in older patients with dementia and consider their exposure to anticholinergic drugs as a predefined predictor.

Design: Electronic health record data within the first $24 \mathrm{~h}$ of admission was collected from 2015-2018. Included were inpatients $\geq 65$ years old, hospitalised $\geq 48 \mathrm{~h}$, diagnosed with dementia according to ICD-10 codes. Outpatients and patients with stays $>24 \mathrm{~h}$ in the intensive care unit were excluded. The outcome was in-hospital mortality. The developed model was based on Naïve Bayes using data from 2015-2016 as training set, the years 2017-2018 served as validation set. The candidate variables were obtained with the Minimum Redundancy Maximum Relevance (MRMR) variable selection algo- rithm and backward selection. Synthetic Minority Oversampling Technique (SMOTE) was applied within the training-set. Performance measures of interest were AUC, sensitivity and specificity. Results: In total, 2,595 patients were included. The training set contained 1266 patients (mean age $83.2 \pm 6.54$ years, $60 \%$ female) of which $63(5.0 \%)$ died during hospitalisation. Variables selected for prediction were: self-care index, delirium observation screening score, sodium, CRP, creatinine, GFR, chronic heart failure, hemi-/paraplegia, COPD, cancer, diabetes and the anticholinergic burden scales Cancelli, ACL, ADS and SCDL. Further, blood pressure, polypharmacy, age, BMI, and temperature were included. A first validation for the final model using the training set showed an AUC of 0.872 , a sensitivity of $90.44 \%$ and a specificity of $68.50 \%$. Using the validation set, we obtained an AUC of 0.771 , a sensitivity of $63.01 \%$ and a specificity of $73.65 \%$.

Conclusion: The developed prediction model for in-hospital mortality showed an acceptable performance. It might be a useful tool to identify patients with dementia with a high risk for in-hospital mortality in order to implement interventions and management strategies during hospitalisation to improve patient outcome, however, further validation is required.

\section{OR01.2}

Undergraduate students and educators' views on interprofessional behaviour change support education in chronic disease

Isa B. Félix ${ }^{1}$, Carla Nascimento ${ }^{1}$, Patrícia Pereira ${ }^{1}$, Katja Braam $^{2}$, Cathal Cadogan ${ }^{3}$, Judith Strawbridge ${ }^{4}$, Leona Cilar ${ }^{5}$, Lucija Gosak ${ }^{5}$, Nuno Pimenta ${ }^{6}$, Mara P. Guerreiro* 1, 7

${ }^{1}$ Nursing Research, Innovation and Development Centre of Lisbon (CIDNUR), Nursing School of Lisbon, Lisbon, Portugal, ${ }^{2}$ inHolland University of Applied Sciences, Haarlem, Netherlands, ${ }^{3}$ Trinity College Dublin, School of Pharmacy and Pharmaceutical Sciences, ${ }^{4}$ Royal College of Surgeons in Ireland, School of Pharmacy and Biomolecular Sciences, Dublin, Ireland, ${ }^{5}$ University of Maribor, Maribor, Slovenia, ${ }^{6}$ Sport Sciences School of Rio Maior, Rio Maior, ${ }^{7}$ Centro de Investigação Interdisciplinar Egas Moniz (CiiEM), Instituto Universitário Egas Moniz, Monte de Caparica, Portugal 
Background and Objective: Interprofessional education (IPE), defined as students from "two or more health/social care professions" learning "with, from and about each other", has been shown to enhance learners' attitudes, knowledge, skills and behaviours for collaborative practice, and, to some extent, clinical outcomes [1]. Inspired by IPE, the Train4Health project aims to improve healthcare students' competencies in behaviour change support for the selfmanagement of chronic disease through an innovative educational package. An international focus group study was conducted to elicit unmet needs and educational product requirements. This paper reports stakeholders' views on interprofessional behaviour change support education in chronic disease.

Method: Eight online focus groups with 39 students and 4 online focus groups with 27 academic educators from pharmacy, nursing and sport science, purposively selected across European countries. Each focus group was conducted in the countries' national languages and recorded. Verbatim transcripts were thematically analysed. Ethical approval was granted by ESDRM.

Main outcome measures: Students and educators' views on interprofessional behaviour change support education in chronic disease.

Results: Data analysis suggests that, with few exceptions, involvement of participants in IPE was still limited. When discussing interprofessional behaviour change education, three broad themes emerged: advantages, barriers and implementation strategies. Collaboration between different professionals was commonly seen as synergistic, enabling students to foster their own role, to envisage future teamwork and to gain knowledge of each other's role. Further advantages expressed were gaining knowledge for practice, patient benefit and concerted action among healthcare professionals. According to the presage-process-product model [1], key barriers included IPE context and teachers' characteristics: lack of an interprofessional team of educators, limited educators' training to provide IPE, curricular diversity across different disciplines, assigning a lower priority to IPE, logistics and insufficient infrastructure.

An implementation strategy that received wide endorsement, although on its own insufficient for students to "learn with, from and about each other", was having academic educators from a range of disciplines. Other strategies included implementing IPE in an early stage of the learning journey, having common modules between different disciplines and establishing institutional partnerships.

Conclusion: Overall, interprofessional behaviour change support education was viewed positively by students and academic educators. Barriers may be mitigated by multimodal strategies, addressing the context (e.g. "top-down" support) and educators' characteristics (e.g. training and up-skilling).

References: 1. Reeves et al. A BEME systematic review of the effects of interprofessional education: BEME Guide No. 39. Med Teach. 2016;38(7):656-68.

\section{OR01.3}

Antimicrobial stewardship program implementation during the global pandemic of coronavirus disease (COVID-19)

\section{Ariana Martínez Suárez* ${ }^{1}$, Elena Salamanca ${ }^{2}$, Natalia Maldonado $^{2}$, Jesús Rodríguez-Baño ${ }^{2}$, Pilar Retamar Gentil ${ }^{2}$}

${ }^{1}$ Clinical pharmacy, ${ }^{2}$ Clinical Infectious Diseases, Hospital Universitario Virgen Macarena, Sevilla, Spain

Background and Objective: High prevalence of antimicrobial prescription has been published in international cohorts of patients admitted for SARS-CoV-2 (between 80 and $40 \%$ ) while the prevalence of bacterial coinfection reported is $<4 \%$ and $3.7-21.9 \%$ for superinfections. The aim of the study is to describe antimicrobial prescriptions in patients admitted for SARS-CoV-2 infection in regards to different levels of implementation of a specific multidisciplinary antimicrobial stewardship program (ASP).

Design: Descriptive study of the use of antimicrobials in a cohort of patients admitted for SARS-CoV-2 infection in a tertiary hospital. The different waves included are the following: first (March 5th-July $31 \mathrm{st}$ ), second (August 1st-January 4th) and third (January 4th-present). The interventions were: A) 1 st and 2 nd wave: discussions of antimicrobials prescriptions by working groups. B) 3rd wave: specific training of antimicrobials use in COVID patients and implementation of a non-compulsory ASP intervention to facilitate adherence to the local recommendations. Frequencies and percentages of monotherapies, combined treatments, different drugs used, prescriptions to treat co-infection (occurring within 48 hours of admission) and superinfections (occurring after 48 hours) are described.

Results: The total percentage of antimicrobial prescription for each wave was: $42.7 \%(n=232), 28.5 \%(n=284)$ and $29.3 \%(n=229)$. The total percentage of antimicrobial prescription in co-infection was: $33.6 \%, 11.6 \%, 11.6 \%$ and in secondary infection was $21.6 \%, 22.9 \%$ and $11.8 \%$. The percentage of use in patients with the only diagnosis of pneumonia was: $33.6 \%, 11.0 \%$ and $6.0 \%$. Frequencies of most prescribed drugs in co-infection were: ceftriaxone $(19.4 \%, 6.6 \%$, $3.9 \%)$, piperacillin/tazobactam $(2.2 \%, 1.0 \%, 3.1 \%)$, amoxicillin/clavulanic $(1.7 \%, 1.4 \%, 0 \%)$ and in secondary infection are: piperacillin/tazobactam $(7.3 \%, 8.5 \%, 3.9 \%)$, ceftriaxone $(5.6 \%, 3.3 \%$ $3.1 \%)$, amoxicillin/clavulanic $(2.6 \%, 2.1 \%, 0.4 \%)$.

Conclusion: The lower prescription rate compared to that reported in international cohorts in our centre might be due to the intense antimicrobial stewardship activities performed. The prevalence of antimicrobial prescription in patients diagnosed with SARS-CoV-2 pneumonia decreased after the implementation of a specific ASP in the third wave.

\section{OR01.4}

ARE pharmacists competent enough to provide patient education about drugs used to treat autism spectrum disorder?

\section{Zekiye Yılmaz $^{*}$, Anmar Al-Taie ${ }^{2}$}

${ }^{1}$ Clinical Pharmacy, Acıbadem Mehmet Ali Aydınlar University, Faculty of Pharmacy, Istanbul, Turkey, ${ }^{2}$ Clinical Pharmacy, Girne American University, Mersin, Cyprus

Background and Objective: Autism is a neurodevelopmental disability typically associated with limitations in social interaction, communication, and behavioral development (1). While autism is widely recognized as an emerging public health problem due to its increasing incidence, pharmacotherapy constitutes an important component of autism treatment $(2,3)$. Given the increasing prevalence of autism and high drug use, the need for pharmacists to be more effective in the treatment of patients with autism cannot be denied. Pharmacists, who are a key player in the field of health, can play an important role in the treatment of individuals with autism by providing patient education and counseling about the drugs used in the treatment of autism (3). The aim of this study is to determine the knowledge of pharmacists about drugs used in the treatment of autism spectrum disorder (ASD) and their tendencies in patient education and counseling in autism spectrum disorder.

Method: A prospective pilot observational study was carried out on community pharmacists all around the Turkey. It was a short-course design from 13 to 20 May 2021. The questionnaire was designed on Google forms and then applied to the participants by sharing the survey link. 
Main outcome measures: Knowledge of pharmacists about dose, dosage form, application time, side effects and drug interactions of medicines used for the treatment of autism. Knowledge of pharmacist about appropriate instructions that should be given to patients / parents when providing the $\operatorname{drug}(\mathrm{s})$ used in the treatment of ASD and trends of pharmacists' about patient education in ASD.

Results: A total of 61 community pharmacists were included in this study with a mean age of $39.05 \pm 13.11$ years constituting $40(65.6 \%)$ females. $15(24.6 \%)$ of pharmacists had a master degree. $41(67.2 \%)$ of the participants declared that they don't know the medicines used for the treatment of ASD. While $45(73,8 \%)$ of the participants stated that they did not know the dosage forms of the drug(s) used in the treatment of ASD, $50(82 \%)$ stated that they not know the appropriate doses. $11(18 \%)$ of the participants declared that they know the appropriate application time of the medicines. $44(72.1 \%)$ of the participants expressed that they did not know the side effects of the drug(s) used in the treatment of ASD, while 53 (86.9\%) expressed that they did not know about drug-drug interactions. It was detected that $45(73,8 \%)$ of the pharmacists do not know the appropriate instructions the pharmacist should give to patients / parents when providing the drug(s) used in the treatment of ASD. 58 (95.1\%) of the pharmacists has never had education about ASD.

Conclusion: The results of this study has shown that community pharmacists need to have more information about drugs used in autism spectrum therapy in order to provide patient education and drug counselling services. The knowledge level and awareness of pharmacists can be increased by organizing in-professional training. References: 1) American Psychiatric Association Diagnostic and Statistical Manual of Mental Disorders (4th ed. Text revised), American Psychiatric Association, Washington, DC (2000)

2) C. Rice, J. Nicholas, J. Baio, et al. Changes in autism spectrum disorder prevalence in 4 areas of the United States. Disabil Health J. 2010;3(3):186-201

3) Khanna R, Jariwala K. Awareness and knowledge of autism among pharmacists. Res Social Adm Pharm. 2012;8(5):464-71.

\section{OR01.5}

A systematic review of interventions to enhance adherence and persistence with ADHD pharmacotherapy

\section{Rebecca Parkin* ${ }^{\text {, Fiona Mc Nicholas }}{ }^{2,}$ 3, 4 , John Hayden ${ }^{1}$}

${ }^{1}$ School of Pharmacy and Biomolecular Sciences, Royal College of Surgeons in Ireland, Dublin, ${ }^{2}$ Children's Health Ireland, Crumlin, Dublin, ${ }^{3}$ Lucena Clinic, Rathgar, Dublin, ${ }^{4}$ Department of Child and Adolescent Psychiatry, School of Medicine and Medical Science, University College Dublin, Dublin, Ireland

Background and Objective: Although high rates of poor adherence/ persistence have been documented in ADHD, $(1,2)$ there is limited research targeting the problem.(3,4) Evaluations of interventions to improve adherence/persistence are rare. This systematic review evaluated interventions that have attempted to address poor adherence/persistence to ADHD pharmacotherapy, with the aim of guiding the development of future interventions.

Method: An extensive search was conducted including the databases of Embase, Pubmed, PsychINFO, CINAHL, the Cochrane Library and Web of Science from January 1980 until January 2021. Only published studies in the English language with full text were included. Studies were also reviewed for quality.

Main outcome measures: The impact of interventions on adherence/ persistence and clinical outcomes in ADHD pharmacotherapy was evaluated.
Results: Thirteen studies were identified involving interventions based on psychoeducation, behavioural therapy, combined psychoeducation/behavioural therapy, technology-based interventions, written informed consent and a nursing support line. All 13 studies (including five RCTs) reported improvement in adherence/persistence and five studies (including four RCTs) also reported improvement in ADHD ratings. Almost all studies involved interventions utilising some form of education. Three RCTs of psychoeducation alone were included, with two of the three studies reporting adherence benefits at three and 12 months respectively. The third RCT was terminated early due to poor recruitment. A behavioural intervention RCT reported improved adherence six months post intervention (but not at 12 months), although a substantial drop out rate was observed. A final RCT included used a Smartphone Application and reported a short term increase in adherence. The quality of studies included for review was typically low. There were few RCTs, a lack of blinding, large drop out rates, poor recruitment, lack of sample size justification, limitations in control group selection and short follow-up periods.

Conclusion: This review has highlighted some potential for interventions in improving adherence/persistence and clinical outcomes in ADHD pharmacotherapy. Future interventions should involve combinations of strategies, have a theoretical framework and target the most common reasons for nonadherence. Interventions should also be integratable into routine care and include patient input to maximise sustainability. To progress adherence intervention research in ADHD, more studies are required to address the recognised limitations of the current evidence base.

References: 1 . Charach, A., A. Ickowicz, and R. Schachar, Stimulant treatment over five years: adherence, effectiveness, and adverse effects. Journal of the american academy of child and adolescent psychiatry, 2004. 43(5): p. 559-567

2. Charach, A. and A. Gajaria, Improving psychostimulant adherence in children with ADHD. Expert Review of Neurotherapeutics, 2008. 8(10): p. 1563-1571

3. Biederman, J., et al., A novel text message intervention to improve adherence to stimulants in adults with attention deficit/hyperactivity disorder. Journal of Clinical Psychopharmacology, 2019. 39(4): p. 351-356

4. Gajria, K., et al., Adherence, persistence, and medication discontinuation in patients with attention-deficit/hyperactivity disorder a systematic literature review. Neuropsychiatr Dis Treat, 2014. 10: p. $1543-69$

\section{OR01.6}

Portrayal of autism spectrum disorder and related treatments in Qatar's printed media

\section{Halima Saadia $^{*}{ }^{1}$, Safeya Habib ${ }^{1}$, Monica Zolezzi ${ }^{1}$ \\ ${ }^{1}$ College of Pharmacy, QU Health, Qatar University, Doha, Qatar}

Background and Objective: Although considerable progress in the diagnosis and treatment of autism spectrum disorder (ASD) has emerged over the last decade, negative media stereotypes about ASD and its treatments are amongst the most hurtful and socially limiting stigma experiences reported by mental health service consumers and family members. Thus, the main objective of this study were to have a better understanding of the written media portrayal of ASD in Qatar, and to evaluate its influence on the public's understanding of ASD and its treatments.

Design: A retrospective, quantitative, and qualitative content analysis of articles printed in Qatar's English and Arabic newspapers over one year was used. Quantitative descriptive analysis was employed to 
examine the extent of ASD media coverage. The qualitative analysis used a pre-determined coding approach derived from an extensive review of the literature to examine the discourse tone and assess the stigmatization of the main messages on the text. Articles discussing ASD treatments were analyzed separately, by reviewing the scientific evidence as outlined in the Qatar and the American Academy of Pediatrics (AAP) ASD treatment guidelines.

Results: A total of 178 ASD-related articles were found in 1 year of published articles. The quantitative analysis revealed that the overall attractiveness of ASD-related articles was poor, the majority were in relation to general news or local events and had a limited focus on the scientific aspects of this condition or its treatments. The discourse analysis revealed significantly more stigmatizing statements in articles in Arabic compared to those published in English newspapers. Based on current practice guideline recommendations, the majority of the ASD treatments discussed had insufficient or lacked scientific evidence.

Conclusion: Results from this study suggest that there is a need to improve how the print media addresses ASD. More scientific and responsible writing is needed, particularly when recommending treatments for this condition.

\section{ORAL COMMUNICATION II}

\section{OR02.1}

Drug-disease interactions in mental illness: clinically relevant alerts for depression and psychotic disorders

Sander D. Borgsteede ${ }^{1}$, Maaike Diesveld ${ }^{*}$, Suzanne De Klerk², Pieter Cornu ${ }^{3}$, Dorothea Strobach ${ }^{4}$, Katja Taxis ${ }^{5}$

${ }^{1}$ Medication Surveillance, ${ }^{2}$ Health Base Foundation, Houten, Netherlands, ${ }^{3}$. Research Group Clinical Pharmacology \& Clinical Pharmacy, Faculty of Medicine and Pharmacy, Vrije Universiteit Brussel, Brussels, Belgium, ${ }^{4}$ Hospital Pharmacy and Doctoral Programm Clinical Pharmacy, University Hospital Munich, Munich, Germany, ${ }^{5}$ Department of Pharmacy, Unit of Pharmacotherapy, Epidemiology and Economics, University of Groningen, Groningen, Netherlands

Background and Objective: Drug-disease interactions are situations where a drug may have negative effects on patients' comorbidities. In these situations, it can be necessary to avoid that drug, adjust its dose or monitor therapy. In the Netherlands, pharmacists have developed a best practice how to evaluate drug-disease interactions and implemented two drug-disease interactions for mental illness in alert systems at the point of care.

The objective of this presentation is to describe the pharmacological background and clinical relevance of alerts for drug-disease interactions for depression and schizophrenia/ psychotic disorders.

Design: We evaluated alerts for drug-disease interactions that were developed by a national, multi-disciplinary expert panel. The alerts were included in the national drug databases and implemented in all clinical decision support systems in primary care and hospitals throughout the Netherlands.

We analysed the number of drugs with proven drug-disease interaction, the number and type of practice recommendation and the underlying pharmacological mechanism.

Results: In total, 17 practice recommendations for 58 drugs were developed for depression as interacting disease, and 5 recommendations for 32 drugs for schizophrenia/ psychotic disorders. The underlying mechanism for depression was the possibility to develop depressive symptoms, and the most common practice recommendation was to closely monitor depressive symptoms, for example in the case of corticosteroids. In patients with schizophrenia/ psychotic disorders the underlying mechanism was the risk of the development of psychosis. For this drug-disease interaction the most common recommendation was to consider possible alternatives. Examples of drugs with alerts in patients with schizophrenia/psychotic disorders were amphetamines and dopamine agonists.

Conclusion: Practice recommendations for two mental illnesses were developed, and implemented in prescribing and dispensing practice in the Netherlands. These recommendations support both pharmacists and physicians in primary care and hospitals by signalling clinically relevant drug-disease interactions at the point of care. Practical advice integrated in clinical decision support assists health care professionals how to solve these drug-related problems, thereby improving medication safety. This practice may be adopted in other settings and contribute to safer medication ue in other countries as well.

\section{OR02.2}

The role of pharmacists in deprescribing-a scoping review

Rikke N. Hansen ${ }^{*}$, Bjarke Abrahamsen ${ }^{1}$, Mira El-Souri ${ }^{1}$, Charlotte V. Rossing ${ }^{1}$

${ }^{1}$ Research and Development, Danish College of Pharmacy Practice, Pharmakon, Hillerød, Denmark

Background and Objective: As experts in medicines, pharmacists play a central role in ensuring safe and effective use of medicines. In recent years, the role of pharmacists has expanded to being a partner in deprescribing inappropriate medicines. With this scoping review we want to explore the role and tasks of pharmacists as part of the deprescribing process. The objective is to review the scope for pharmacists' involvement in deprescribing in primary and secondary health care.

Method: A scoping review was conducted using queries in the PubMed database (2015-2020) with search words identified through a PICO analysis. We further identified relevant literature and ongoing projects by searching grey literature and reaching out to national and international pharmacy care networks. All identified literature was screened by the authors according to a set of criteria.

Main outcome measures: This scoping review explored which tasks pharmacists have in deprescribing of medicine and how pharmacists collaborate with patients and physicians in deprescribing.

Results: From PubMed, 387 studies were identified, and 18 studies were included. From grey literature and pharmacy care networks, five studies or projects were included. A review of the included studies shows that pharmacists collaborate with patients, physicians, and other health care professionals in the deprescribing process and may be responsible for any part of the process, such as screening, assessment, prioritising, planning, initiation and follow-up. In all but two studies pharmacists identified candidates for deprescribing by screening for inappropriate medicines followed by assessment. Five studies described how pharmacists can use an approved protocol to deprescribe with authorisation by the prescribing physician. The review also identified five studies in which pharmacists provided specific information about deprescribing to either health care professionals or patients. Finally, the involvement of pharmacists in deprescribing can take place in both primary and secondary health care settings.

Conclusion: Pharmacists contribute to deprescribing in close collaboration with physicians and patients in primary and secondary health care. 


\section{OR02.3}

Exploring non-prescribing hospital pharmacists' views of pharmacist prescribing in hospital settings

\author{
Shane Kearney ${ }^{1}$, Jennifer Fawcett ${ }^{2}$, Kieran Dalton ${ }^{*} 1$ \\ ${ }^{1}$ Pharmaceutical Care Research Group, School of Pharmacy, \\ University College Cork, Cork, ${ }^{2}$ Pharmacy Department, St. Vincent's \\ University Hospital, Dublin, Ireland
}

Background and Objective: Despite the successful implementation of hospital pharmacist prescribing in some countries, there is limited qualitative research that has evaluated in depth stakeholders' perceptions of pharmacist prescribing in hospitals prior to its implementation. Therefore, the objective of this study was to explore the topic of hospital pharmacist prescribing with non-prescribing hospital pharmacists in Ireland, a country where pharmacist prescribing is not commonplace.

Method: Semi-structured interviews were conducted with pharmacists from two acute university teaching hospitals in the Munster region of Ireland without any pharmacist prescribing, and were sampled based on their years of post-qualification experience. The interviews were audio-recorded, transcribed verbatim, and subsequently underwent thematic analysis (facilitated by NVivo ${ }^{\circledR} 12$ ). Ethics approval was obtained prior to study commencement.

Main outcome measures: Views of non-prescribing hospital pharmacists on pharmacist prescribing in hospitals.

Results: Ten pharmacists were interviewed between September and October 2019. The mean number of years of post-qualification experience was nine. The mean interview length was 37 minutes (range 25-48 $\mathrm{min}$ ). Three major themes were generated from the data:

1. Advanced responsibility within pharmacists' scope of expertise: pharmacists expressed frustration at feeling underutilised to personally rectify errors that they identified, and welcomed undertaking prescribing responsibility to provide better patient care-which would also increase job satisfaction. There was no clear consensus on the best prescribing model to use, but participants highlighted the importance of defining pharmacists' scope of prescribing and knowing limitations.

2. Impact on healthcare provision: it was emphasised that pharmacist prescribing would allow for swifter medication optimisation and reduce the number of times that pharmacists had to contact doctors, which may decrease workload and improve staff working relationships. Although pharmacist prescribers may encroach on other prescribers' roles, it was perceived that they may be more cost-effective, reduce organisational litigation, and ultimately enhance patient safety.

3. Supporting the implementation: legislative change alongside the development of the hospital pharmacist career structure are needed to recognise the advanced role of pharmacist prescribers. Clear organisational policies are required, whilst good support from other hospital stakeholders was perceived as a strong facilitator - particularly from doctors, as they may supervise prescribing training. Hospitals will also need to provide the financial backing to facilitate training, protected time off, and additional staff.

Conclusion: This exploratory study has shown primarily positive views towards pharmacist prescribing from non-prescribing pharmacists in Irish hospitals. The interviewees perceived that pharmacist prescribing authority in hospitals would be beneficial to patient care delivery, but further work is needed to establish a defined scope of practice and the relevant supports required prior to its routine implementation.

\section{OR02.4}

How does knowledge of multimorbid patients' priorities influence the outcomes of medication reviews?

Viktoria S. Wurmbach ${ }^{* 1,2}$, Marcel K.-P. Kusch ${ }^{1,2}$, Friederike H. Böhlen $^{3}$, Walter E. Haefeli ${ }^{1,2}$, Beate Wild ${ }^{3}$, Hanna M. Seidling ${ }^{1,2}$

${ }^{1}$ Department of Clinical Pharmacology and Pharmacoepidemiology, ${ }^{2}$ Cooperation Unit Clinical Pharmacy, ${ }^{3}$ Department of General Internal Medicine and Psychosomatics, Heidelberg University Hospital, Heidelberg, Germany

Background and Objective: The 'Life and Vitality Assessment' (LAVA) is an instrument for a two-step evaluation of patient priorities: Patients are first asked to prioritize different aspects of their lives (e.g., family or mobility) to identify those that are most important to them. Patients then rate their current satisfaction with these aspects. The objective of this work was to evaluate whether information obtained through the LAVA will influence medication reviews by health professionals.

Method: A total of 20 patient cases from the PACT study (German Ministry of Education and Research; grant: 01GL1728) were purposefully selected by the authors based on the expected need for a medication review (e.g., high number of drugs or diagnoses). Two reviewers of a team of four pharmacists and one physician were asked to independently review each patient's medication in order to identify potential medication-related problems, and then to prioritize the topics that they would hypothetically address with the respective patient, e.g., unanswered questions or suspected needs for intervention. To do so, reviewers were provided with the medication, the diagnosis, and a short introduction on the patient's background, and were recommended the German guideline on multimedication as support ${ }^{1}$. After completing the review, the reviewers were additionally provided with information obtained by the LAVA and asked to re-evaluate their prior assessment. Finally, two members of the study team independently assessed the reviewers' agreement on the topics they would address by assigning one point for each agreement between the two reviewers within the three highest prioritized topics. Hence, two distinct scores (ranging from 0 to 3 ) that both mirrored the reviewers' agreement in each patient case could be obtained - one without and one with LAVA information.

Main outcome measures: The reviewers' agreement before and after receiving the LAVA information was determined. Moreover, the number of patient cases for which LAVA information led to changes in the reviewer's evaluation was quantified. Differences between reviewers' agreement were tested by using the Wilcoxon signed-rank test.

Results: The LAVA information increased the reviewers' agreement in eight cases (40\%); only in one case the agreement decreased as measured by the score (average score without LAVA information $( \pm \mathrm{SD}): 1.10( \pm 0.85)$, average score with LAVA information ( \pm SD): 1.65 ( \pm 0.88$) ; \mathrm{p}=0.027)$. In 17 of 20 patients $(85 \%)$, the provision of LAVA information resulted in a re-evaluation by at least one reviewer and in 12 cases $(60 \%)$ in a revision by both reviewers, mainly by adjusting the topics to be addressed to the aspects patients were particularly unsatisfied with.

Conclusion: When LAVA information was provided, the action and intervention needs discovered by health professionals based on a medication review were less variable, suggesting that they might help to identify and prioritize potential problems.

References: 1. Leitliniengruppe Hessen, Deutsche Gesellschaft für Allgemeinmedizin und Familienmedizin (DEGAM), PMV forschungsgruppe, et al. Hausärztliche Leitlinie Multimedikation. Empfehlungen zum Umgang mit Multimedikation bei Erwachsenen und geriatrischen Patienten. Version 1.09. 2014. Available from: 
https://www.degam.de/degam-leitlinien-379.html [Accessed 10th June 2021].

\section{OR02.5}

Pharmacists' role in medication reconciliation, the first application in a Vietnamese hospital: a cross-sectional study

\section{Khanh Hoang Phuong Nguyen* 1 , Huy Q. Le Tran², Ngoc Bui} Hong', Thuy T. T. Ngo", Dung T. Nguyen $^{2}$

${ }^{1}$ Pharmacy, Vinmec Central Park hospital, ${ }^{2}$ Department of Clinical Pharmacy, School of Pharmacy, University of Medicine and Pharmacy at Ho Chi Minh City, Ho Chi Minh, Viet Nam

Background and Objective: Medication reconciliation plays a vital role in reducing medication errors and decreasing drug-related problems at transitions of care. In Vietnam, the medication reconciliation process is not broadly applied, and there is a lack of published data about this process

Method: STUDY DESIGN: Cross-sectional study among patients admitted into one of three wards including General Internal Medicine, Cardiovascular and Intensive Care Unit at Vinmec Central Park International Hospital from February 2020 to July 2020

METHODS: A form was designed to collect patient data, including patients' best possible medication history. Pharmacists' interventions from the medication reconciliation process were classified according to the PCNE classification system. The factors associated with successful interventions were determined by logistics regression to analyze.

Main outcome measures:

- The characteristics of reconciled patients

- The role of pharmacists in reconciliation activity

- Factors related to pharmacist's interventions

Results: Among 182 patients included in this study, 149 patients had their medications reconciled. The majority of patients were interviewed by clinical pharmacists within $24 \mathrm{~h}$ of admission, and the average duration of an interview was 15-30 min. Among these patients, $65 \%$ could provide at least two sources of information to confirm their medication history. Although $90 \%$ of patients adhered to their medication regimen, only $1 \%$ fully understood their medications. Additionally, $83 \%$ of patients had at least one medicationrelated risk (chronic medication use, changing more than four drugs during hospitalization, etc.). The rate of pharmacists' intervention from unintended discrepancies was 0.8 interventions per patient, $90 \%$ of which were accepted and fully implemented by doctors or patients. High-alert medications usage (OR 2.63, 95\% CI 1.20-5.79; $\mathrm{p}=0.016$ ) and number of medications used before admission (OR 1.21, CI $1.07-1.37 ; \mathrm{p}=0.002$ ) may contribute to successful interventions.

Conclusion: Medication reconciliation led by pharmacists could improve the patient's safety and optimize their treatment at transitions of care.

\section{OR02.6}

Mental heatlh evaluation in community pharmacy: results of a pilot study

Mónica Condinho* 1, 2, Isabel Ramalhinho ${ }^{2}$, Ana Guarda ${ }^{1}$, Catarina Vaz Velho ${ }^{3}$, Carlos Sinogas ${ }^{1,4}$, Margarida Moniz ${ }^{5}$, Kevin Rodrigues ${ }^{6}$, Catarina Rodrigues ${ }^{7}$

${ }^{1} \mathrm{ACF}$ - ACOMPANHAMENTO FARMACOTERAPÊUTICO, LDA, Évora, ${ }^{2}$ Faculty of Sciences and Technology, University of Algarve, Faro, ${ }^{3}$ Department of Psychology, ${ }^{4}$ Department of Medical and Health Sciences, University of Évora, Évora, ${ }^{5}$ Pharmacy Algarve, S. B. de Messines, ${ }^{6}$ Pharmacy Central, Mora, ${ }^{7}$ Pharmacy Albufeira, Albufeira, Portugal

Background and Objective: There is a high prevalence of mental illness in Portugal. The COVID-19 pandemic has aggravated the situation. Community pharmacists are well placed to screen and help in the treatment of people with anxiety and depression.

The aim of this study was to screen undiagnosed people for anxiety and depression symptoms and to assess the control of these conditions in diagnosed patients.

Method: Observational, descriptive, and transversal study of a sample of community pharmacy users, selected by a non-probabilistic method, by the pharmacy team (real-world study). Aged 18 years or over, availability to fill the questionnaire (self-administered), subscribed the informed consent and cognitive ability to understand the study were the inclusion criteria. The questionnaire included sociodemographic data, diagnosed diseases, scales to assess anxiety (Generalized Anxiety Disorder-7, GAD-7) and depression (Patient Health Questionnaire-9, PHQ-9) and questions related to the impact of the COVID-19 pandemic in participants' life. Statistical analysis was performed using SPSS (IBM SPSS V. 25).

Main outcome measures: Anxiety and depression scales scores.

Results: Between September and November 2020, a total of 139 participants, 116 females $(81.3 \%)$ were included. The mean age was $52.5 \pm 14.8$ years. Hypertension $(34.5 \%)$, depression $(30.2 \%)$, pain $(26.7 \%)$, anxiety $(25.9 \%)$ and insomnia $(17.3 \%)$ were the most reported diseases.

Of the undiagnosed participants (82), $14(17.1 \%)$ and $11(13.4 \%)$ revealed moderate or severe symptoms of anxiety and depression, respectively. From the patients with diagnosed anxiety (36), 16 (44.4\%) reported moderate to severe symptoms. From the patients with diagnosed depression (42), 19 (45.2\%) reported moderate to severe symptoms.

It was found that moderate or severe symptoms of anxiety are higher in females $\left(\chi^{2}=8.820 ; \mathrm{p}=0.012\right)$, among those taking medication for insomnia $\left(\chi^{2}=6.012 ; \mathrm{p}=0.049\right)$, anxiety $\left(\chi^{2}=8.751\right.$; $\mathrm{p}=0.013)$ and depression $\left(\chi^{2}=18.615 ; \mathrm{p}<0.001\right)$. Moderate or severe symptoms of depression are more frequent in people who live alone and take medication for depression (Fisher exact, $p=0.003$ ) and for anxiety (Fisher exact, $\mathrm{p}=0.003$ ).

Regarding the impact of the COVID-19 pandemic on the participants' lives, professional $(70 ; 50.4 \%)$ and family $(47 ; 33.8 \%)$ were domains where participants reported a more negative impact.

Furthermore, on a numeric scale between 0 (no impact) and 10 (serious harm), the respondents indicated a medium to high impact (5.7 \pm 3.04 on average; median 6.0). Higher impact was found among participants with higher anxiety scores (Fisher exact test; $\mathrm{p}=0.034$ ). Conclusion: Our results showed that pharmacists can make a significant contribution to early identification of people at risk for anxiety and depression. Additionally, among those previously diagnosed, the need for better control is identified, anticipating the contribution of clinical pharmacist services. 


\section{ORAL COMMUNICATION III}

\section{OR03.1}

"I just thought that it was such an impossible thing": a qualitative study of barriers and facilitators to discontinuing long-term use of benzodiazepine receptor agonists using the theoretical domains framework

\author{
Tom Lynch* ${ }^{*}$, Cristín Ryan ${ }^{2}$, Cathal A. Cadogan ${ }^{2}$ \\ ${ }^{1}$ The School of Pharmacy and Biomolecular Sciences, Royal College \\ of Surgeons in Ireland, ${ }^{2}$ The School of Pharmacy and Pharmaceutical \\ Sciences, Trinity College Dublin, Dublin, Ireland
}

Background and Objective: Existing interventions to reduce longterm benzodiazepine receptor agonist (BZRA) use ( $>3$ months) in primary care lack theoretical underpinning and detailed descriptions ${ }^{1}$. This creates difficulties in understanding how interventions work and how they can be replicated in practice. The Theoretical Domains Framework (TDF) can be used to identify behavioural determinants to target when developing behaviour change interventions ${ }^{2}$. This study aimed to explore barriers and facilitators to discontinuing long-term BZRA use from the perspective of both current and previous users.

Method: Semi-structured interviews were conducted with individuals who met the following inclusion criteria: current or previous experience of long-term BZRA use; $\geq 18$ years old; community-dwelling in the Republic of Ireland. A multi-strand convenience sampling method was used to recruit eligible participants involving community pharmacies, general practices and social media. Interview topic guides were developed using the TDF (14-domain version) and explored participants' previous/current BZRA use and perceived barriers and facilitators to discontinuing long-term BZRA use. Data were recorded, transcribed verbatim and analysed using the framework method.

Main outcome measures: Participants' perceptions of barriers and facilitators to discontinuing long-term BZRA use.

Results: Twenty-eight patients were interviewed (13 previous users, 15 current users). Despite commonalities in perceived barriers/facilitators to discontinuing BZRAs, individual participants had different experiences of identified determinants of BZRA discontinuation. For example, both similarities and differences existed within and between each group in terms of knowledge of appropriate duration of BZRA use ('Knowledge'), experience of withdrawal symptoms ('Reinforcement') and availability of resources/supports for discontinuation ('Environmental context and resources'). Compared to previous users, more barriers and fewer facilitators of BZRA discontinuation were identified among current users.

Conclusion: This study reports the barriers and facilitators to discontinuing long-term BZRA use from the perspectives of current and previous users. The findings highlight the challenging nature of BZRA discontinuation and multitude of barriers that impact upon behaviour. Future work will look to develop a theory-based intervention to support BZRA discontinuation in primary care.

References: 1. Lynch et al. Addiction. 2020;115(9):1618-39.

2. Cane et al. Implementation Science. 2012;7(1):37.

\section{OR03.2}

Development and application of an intelligent internet management platform for patients with cancer pain

Jian Xiao* ${ }^{1}$, Lu Zhang ${ }^{1}$, Hang-Xing Huang ${ }^{1}$, Ya-Min Huang ${ }^{1}$,
Ling Huang

${ }^{1}$ Pharmacy, Xiangya Hospital, Central South University, Changsha, China

Background and Objective: Motivated by the need for better pain management, an Internet platform was build with an intelligent decision-making model, and multi-disciplinary team (MDT) cooperation was carried out to provide cancer pain patients with standardized pain management from in-hospital assessment to out-ofhospital follow-up. A randomized controlled trial was designed to evaluate whether the platform would provide better pain management. Method: The comprehensive screening and evaluation process developed according to the three-step analgesia principle and NCCN guidelines for cancer pain, combined with the outpatients' titration plan and evidence-based recommendations to design an easy-to-operate Internet platform for cancer pain medications. Patients were enrolled from tumor chemotherapy, radiotherapy, respiratory medicine, veteran respiratory medicine, breast surgery and pain clinics and randomly assigned to the intervention group (InterG ) or the control group (CTLG). For patients in the InterG, The MDT would review demographic information, assess pain, conduct medication therapy reviews, offer targeted education and make medication plans through the platform, and would solve medication-related problems for patients in home setting. For patients in the CTLG, they just received traditional outpatient services and medication education in the clinic. The relevant information was collected in the fourth week. Main outcome measures: The primary outcome included pain intensity. Secondary outcomes included medication adherence and ADRs.

Results: The pain management platform "Medicine Butler" was established, including a module to collect patients' basic information, a module embedded with Patient-Reported Outcome (PRO) scale to assess pain, a module to offer a medication decision and medication education. The platform established a decision chain of "patient condition collection-intelligent recognition of drug related problemsintelligent medication plan", and achieved real-time responses to patients. We included a total of 100 patients, with 50 in each group. At the fourth week, the worst and average pain score were statistically different, with the median values of 4.0 (IQR, 2.0-5.0) and 6.0 (IQR, 4.0-9.0) ( $\mathrm{P}=0.001)$, and 2.0 (IQR, 1.0-4.0) vs. 4.0 (IQR, 3.0-6.0) (P $=0.001)$, respectively. The rate of medication adherence of the InterG increased from $40.1 \%$ to $60.8 \%$, while that in the CTLG increased from $35.7 \%$ to $43.0 \%$ ( $\mathrm{P}<0.001$ ). More ADRs were monitored in the InterG $(\mathrm{P}=0.003)$.

Conclusion: The result supports the feasibility of intelligent decisionmaking and management of cancer pain medication through a digital platform. It could not only promote rational medication and chronic diseases management, but also reflect the value of pharmacists and quality of hospital services. The platform improved pain control, enhanced medication adherence, and easier to monitor ADRs. 


\section{OR03.3}

Reflection on a patient support approach by the hospital pharmacist: the example of the pathway of the patient being discharged with antibiotic therapy by linezolid during an osteoarticular infection

\section{Hami Narjesse ${ }^{*}$ 1, 2 , Pauline LAZARO ${ }^{2}$, Gwenael LE MOAL ${ }^{3}$, Pierre PRIES $^{4,5}$, Guillaume BINSON ${ }^{2,4}$}

${ }^{1}$ School of pharmacy, Bordeaux University, Bordeaux, ${ }^{2}$ Pharmacy, ${ }^{3}$ Division of infectious diseases, Poitiers teaching hospital, ${ }^{4}$ School of medicine and pharmacy, Poitiers University, ${ }^{5}$ Orthopedic surgery, Poitiers teaching hospital, Poitiers, France

Background and Objective: Osteoarticular infections (OAI) require months-long antibiotic therapy to recover. In this context, hospital pharmacists play a key role to assist outpatient in reaching proper drug compliance for therapeutic effectiveness and to avoid the emergence of multi-resistant bacteria. The objective of our study was to promote drug compliance of antibiotics by the implementation of a specific pharmaceutical pathway for outpatients suffering of OAI. Design: A multidisciplinary team (clinicians, pharmacists, nurses) was set up to define the implementation of the specific pharmaceutical pathway. Critical points of patient pathways in our hospital were identified to propose adequate pharmaceutical interventions. Ambulatory settings were also included. A period of overall testing was defined to assess the feasibility of the process.

Assessment of the impact of pharmaceutical intervention was made by the evaluation of medications discrepancies, drug interactions, adverse effects, therapeutic efficiency, and patient satisfaction at each stage of care.

Results: The specific pharmaceutical care pathway was divided in four steps:

1) Medication collection and review (MC \& MR), made by the pharmacy student after surgery.

2) Pharmaceutical interviews: antibiotherapy's details and answer to patient's questions: 55 patients were interviewed between August 2020 and March 2021. We noticed 7 cases of unintentional discrepancies $(12 \%)$ thanks to the MR. Among them, 4 pharmaceutical interventions were carried out on DI with Linezolid: 3 with Tramadol and 1 with Amitriptylin; leading to their suspension with a more suitable alternative. A summary letter was systematically sent to the pharmacy and referring doctor.

3) Outpatient use dispensation: 10 patients were discharged on Linezolid (18\%) with CRIOGO (Reference Centre for Osteoarticular Infections of the Great West), information sheets (adverse effects, DI ...), as well as an adapted biological monitoring booklet.

4) Pharmaceutical telecare: 7 patients have been recalled after 6 weeks which revealed any adverse effects and an overall patient satisfaction.

Conclusion: Evolution from a static to a dynamic and integrative model of clinical pharmacy through different stages allowed to improve pharmaceutical care of outpatient suffering from OAI with a full coverage of their care pathways. Now, our challenge is to develop this model to other outpatients requiring long-term treatments, such as oral chemotherapies or other antibiotics treatments.

\section{OR03.4}

Risk stratification of patients for postoperative complication after knee or hip arthroplasty-preliminary results

Petr Domecky ${ }^{*}{ }^{1}$, Anna Rejman Patkova ${ }^{1}$, Pavel Sponer ${ }^{2}$, Tomas Kucera $^{2}$, Josef Maly ${ }^{1}$

${ }^{1}$ Department of Social and Clinical Pharmacy, Faculty of Pharmacy in Hradec Kralove, Charles University, ${ }^{2}$ Department of Orthopaedics, University Hospital Hradec Kralove, Hradec Kralove, Czech Republic

Background and Objective: Surgical site infection is a potential complication of all surgical procedures. There is double mortality in patients with developed infection who underwent a surgical procedure compared to non-infected patients. This study aimed to stratify patients according to the risk of postoperative infection and verify the findings in clinical practice.

Method: This prospective study has started in March 2020 at the Department of Orthopaedics, University Hospital Hradec Kralove. The study included patients aged $\geq 18$ years who underwent primary total hip or knee arthroplasty and signed up for informed consent. Exclusion criteria were American Society of Anaesthesiologist (ASA) score higher than IV and patients with clinical signs of infection, neoplasia, or inflammatory diseases (rheumatoid arthritis, Crohn's disease, HIV, etc.).

Main outcome measures: Inflammatory markers, especially neutrophil-to-lymphocyte ratio (NLR), prognostic inflammatory and nutritional index (PINI) and intensive care infection score (ICIS), were analysed one day before surgery (-1D), two days after the surgery (2D) and during outpatient check after discharge (OC). Furthermore, postoperative complications were evaluated by orthopaedists with appropriate laboratory and physical examinations.

Results: 42 patients (16 women and 26 men) with an average age of $64.04 \pm 10.21$ years were included in the study. Hip arthroplasty was performed in $31(73.8 \%)$ and knee arthroplasty in $11(26.2 \%)$ patients. Cefazolin was used in $88.1 \%$ and vancomycin in $11.9 \%$ of surgeries. Cefazolin was administered as one dose before incision, the second dose 4 hours after the first dose and the third and the fourth dose with a dosing interval of 6 hours. Vancomycin was administered as one dose before incision and the second dose after surgery with a dosing interval of 12 hours. -1D: NLR $>4$, PINI score $>21$ and ICIS $>4$ was identified in 5, 0 , and 1 patient respectively. $2 \mathrm{D}$ : NLR $>4$, PINI score $>21$ and ICIS $>4$ was identified in 14, 24, and 4 patients respectively. OC: NLR $>4$, PINI score $>21$ and ICIS $>4$ was identified in no patient at all. ACS (American Society of Surgeons) risk score $>5 \%$ was identified in 9 patients. In addition to the ACS risk score, ASA score = III was identified in 17 patients. The postoperative infection was identified in $2(4.7 \%)$ patients, postoperative anaemia in $12(28.6 \%)$ patients, pulmonary embolism in $2(4.7 \%)$ patients and other infection (urinary) in $1(2.3 \%)$ patient. The average length of hospitalisation was $11 \pm 6.7$ days.

Conclusion: Increased inflammatory markers could be suitable for detecting early postoperative infection or other postoperative complications. However, a more extensive set of patients is needed for further detailed statistical analysis with sensitivity, specificity, and receiver operating characteristic curve. 


\section{OR03.5}

Guideline adherence among health professionals for cardiovascular and metabolic monitoring of patients prescribed antipsychotic medications: evaluation of data from primary care

\author{
Ruba Azfrali* $^{*}$, ${ }^{2}$ Zahraa Jalal $^{1}$, Vibhu Paudyal ${ }^{1}$ \\ ${ }^{1}$ School of Pharmacy, University of Birmingham, Birmingham, \\ United Kingdom, ${ }^{2}$ Clinical pharmacy department, Umm Al-Qura \\ University, Makkah, Saudi Arabia
}

Background and Objective: Despite their known effectiveness, antipsychotics are known to possess important cardiometabolic adverse event profiles. Guidelines emphasise routine monitoring, however practices have been suggested to be suboptimal. This study aims to investigate the level of guideline adherence among health care professionals for cardiovascular and metabolic monitoring for patients prescribed antipsychotic medicines in primary care settings.

Method: Data were collected for patients diagnosed with mental illness and prescribed antipsychotic medications at two primary healthcare (general practices) services in England over a five years period (February 2016-February 2021).

Main outcome measures: The main outcome measures were the proportion of patients with evidence of monitoring for cardiometabolic parameters (body compositions, anthropometrics, lipids, glucose outcomes). Univariate and multivariate regression models were used to analyse the characteristics of the profiles and to explore the factors associated with the monitoring practices.

Results: A total of 1628 records of patients prescribed antipsychotic medications were included for the analysis. The proportion of patients who received cardiometabolic monitoring at least once yearly varied across different parameters. Patients were mostly monitored for BP (92\%), body weight \& BMI (over 85\%) and HDL (72\%), but to a lesser extent for other lipid parameters (LDL \& total cholesterol $\approx 2 \%$ ) and blood glucose $(\approx 2 \%)$. In the multivariable analysis, patient factors mainly old age, pre-existing comorbidities (CVD and $\mathrm{DM})$ were significantly associated with better monitoring practices for all cardiometabolic parameters. On the contrary, the use of antipsychotic agent with high metabolic risk (olanzapine) and psychotropic polypharmacy were not associated with improved monitoring practices. Similarly, abnormal cardiometabolic values such as dyslipidaemia did not report to improve monitoring practices.

Conclusion: In general, annual follow-up cardiometabolic monitoring was infrequent, irregular, and did not change in response to abnormal test results nor to the use of antipsychotic agents with high cardiometabolic risks. More efforts are needed to improve the adherence to cardiometabolic monitoring guidelines for antipsychotic drugs users including further roles of clinical pharmacists in the monitoring process.

\section{OR03.6}

Factors associated with antipsychotic prescribing in multimorbid older adults and risk of drug-related readmissions

Adeline Bienfait* ${ }^{1}$, Juliette Lagreula ${ }^{2}$, Manuel R. Blum ${ }^{3}$, Nicolas Rodondi $^{4}$, Bastiaan Sallevelt ${ }^{5}$, Wilma Knol ${ }^{6}$, Denis O'Mahony ${ }^{7}$, Anne Spinewine ${ }^{8}$, Benoit Boland', Olivia Dalleur ${ }^{1}$

${ }^{1}$ Pharmacy department, Cliniques Universitaires Saint Luc, ${ }^{2}$ Louvain, Louvain Drug Research Institute, Clinical Pharmacy Research Group, Université catholique de Louvain, Brussels, Belgium, ${ }^{3}$ Department of General Internal Medicine, Inselspital, Bern University Hospital,

${ }^{4}$ Institute of Primary Health Care (BIHAM), University of Bern, Bern, Switzerland, ${ }^{5}$ Pharmacy department, ${ }^{6}$ Geriatric department,
University medical center, Utrecht, Netherlands, ${ }^{7}$ Geriatric department, Cork University Hospital, Cork, Ireland, ${ }^{8}$ Pharmacy department, Centre Hospitalier Universitaire UCL-Namur, Namur, ${ }^{9}$ Geriatric department, Cliniques Universitaires Saint Luc, Brussels, Belgium

Background and Objective: Some findings, mainly on patients with specific pathologies (e.g. dementia) or institutionalized, suggest that the use of antipsychotics is related to patients'characteristics. However, there are limited data on general multimorbid older adults, while $15 \%$ of patients over 65 have an antipsychotic medication.

The aims are (1) to identify factors associated with antipsychotic prescribing in hospitalised older adults, (2) to assess whether antipsychotic prescribing increases the risk of all-causes drug-related readmissions (DRA) within a year and (3) to describe cases of antipsychotics-related admissions.

Design: This is a secondary analysis of the "OPtimising thERapy to prevent Avoidable hospital admissions in the Multimorbid elderly" (OPERAM) trial, which evaluated the impact of a systematic structured medication review on DRA on patients over 70, multimorbid ( $\geq 3$ chronic medical conditions) and polymedicated ( $\geq 5$ chronic medications). An expert team assessed DRA using an adjudication tool during the OPERAM trial.

Baseline characteristics and comorbidities have been included in a multivariate binary logistic regression to detect factors associated with antipsychotics prescribing and DRA.

Results: 7,7\% (154/2008) of patients had antipsychotics at any time of index hospitalisation. Most prescribed medications were quetiapine $(n=152)$, haloperidol $(n=48)$ and risperidone $(n=22)$, used at low dose $($ mean PDD/DDD ratio $=0,37)$, for indications other than psychosis (insomnia, agitation, depressive disorder).

In multivariate analysis, dementia ( $\mathrm{OR}=3,72895 \% \mathrm{IC}[2,24 ; 6,20])$, psychosis $(26,191[7,39 ; 92,80])$, delirium $(6,407[3,80 ; 10,81])$ and mood disorders $(2,603[1,65 ; 4,11])$ increased the likelihood of antipsychotic prescription. Lower alcohol consumption $(2,204$ [1,36; $3,57])$, lower ADL scores $(3,961[2,55 ; 6,15])$ and higher number of drugs $(1,666[1,36 ; 3,57])$ were positively associated with antipsychotic prescribing.

In this population, antipsychotic prescribing was not associated with an increased risk of all-causes DRA within a year $(0,966[0,65$; 1,42]). However, 36/ 154 patients (23\%) had at least one DRA within a year and in 8 of them $(22 \%)$ the antipsychotic medication was a causing or contributing factor. Falls $(n=3)$ and confusion $(n=3)$ were the main readmission reasons.

Conclusion: In this multimorbid polymedicated older population, antipsychotics were used unfrequently, mainly at low dosage, and unsurprisingly associated with neuro-psychiatric comorbidities, higher number of drugs and lower functional status.

\section{POSTER DISCUSSION FORM I}

\section{PDF01.1}

Development, content validation and pilot testing of the antipsychotics in dementia attitude questionnaire (ADAQ)

Amna Raza ${ }^{*}$, Parastou Donyai ${ }^{1}$, Sundus Jawad ${ }^{2}$, Tim Langran ${ }^{1}$, 2

${ }^{1}$ University of Reading, Reading, ${ }^{2} \mathrm{CCG}$, East Berkshire , United Kingdom

Background and Objective: Despite significant warnings of adverse effects with using antipsychotics in dementia, these continue to be prescribed for the management of behavioural and psychological symptoms of dementia (BPSD), especially in care homes. Staff 
looking after residents in these settings have a significant degree of influence on the use of these medicines for BPSD (1). Therefore, it is imperative to understand how staff within this sector view the risks and benefits of using antipsychotics for BPSD, for example through theory-based attitude questionnaires. The aim was to develop, validate, and pilot a theory-based tool to measure care-staff views about the use of antipsychotics in dementia.

Method: An 81-item questionnaire, the Antipsychotics in Dementia Attitude Questionnaire (ADAQ-v1), was constructed based on the Theory of Planned Behaviour (2) by modifying an existing, but incomplete Dutch questionnaire (3). Eleven staff in two UK care homes were recruited for content validity with the resultant second version (ADAQ-v2) pilot tested with a further 18 care-home staff to generate the finalised version (ADAQ-vf). The content validity index (CVI) was calculated for each item of ADAQ-v1 (4). Principal Component Analysis (PCA) and Cronbach's alpha were used to determine the validity and reliability of ADAQ-v2. The study received approval from the University's Ethics Committee (UREC 19/38).

Main outcome measures: To develop and determine the psychometric properties of the ADAQ questionnaire.

Results: A total of 21 from 81 items of ADAQ-v1 had an item CVI (I-CVI) $<0.78$ and were deleted, based on feedback 12 items were reworded and reduced to 5 items and 6 questions added leaving ADAQ-v2 with 59 items. The PCA for ADAQ-v2 showed 47 items with a factor loading $\geq 0.5$ and $\mathrm{KMO}>0.5$, with the remaining nine items deleted and retained 3 demographic questions. Cronbach's alpha showed high internal consistency $(\alpha=\geq 0.6)$ within the 47 items. Thus, the finalised draft ADAQ-vf contained 50 items including 3 demographic questions.

Conclusion: A validated and theory-oriented tool was developed to measure staff attitudes towards the use of antipsychotics in residents with BPSD. The questionnaire can be used by health and social care professionals to gauge the views of staff caring for residents with dementia in care homes.

\section{References:}

1.Mavrodaris A, Philp I. Reducing antipsychotic prescriptions in primary care: a healthcare perspective. Journal of Public Mental Health. 2013 Mar 15.

2.Ajzen I. The theory of planned behavior. Organizational behavior and human deci-sion processes. 1991 Dec 1;50(2):179-211.

3.Janus SI, van Manen JG, IJzerman MJ, Bisseling M, Drossaert $\mathrm{CH}$, Zuidema SU. Determinants of the nurses' and nursing assistants' request for antipsychotics for people with dementia. International psychogeriatrics. 2017 Nov 21;29(3):475-84.

4.Polit DF, Beck CT, Owen SV. Is the CVI an acceptable indicator of content validity? Appraisal and recommendations. Research in nursing \& health. 2007 Aug;30(4):459-67.

\section{PDF01.2}

Prevalence and correlates of potentially inappropriate medication use in older adults in different settings of care in the Czech Republic

\section{Jovana Brkic $^{*}$, Jindra Reissigova ${ }^{2}$, Daniela Fialova ${ }^{1,3}$}

${ }^{1}$ Department of Social and Clinical Pharmacy, Faculty of Pharmacy in Hradec Kralove, Charles University, Hradec Kralove, ${ }^{2}$ Department of Statistical Modelling, Institute of Computer Science, Czech Academy of Sciences, ${ }^{3}$ Department of Geriatrics and Gerontology, 1st Faculty of Medicine, Charles University, Prague, Czech Republic

Background and Objective: Prescribing medications for older adults is challenging due to high interindividual variability in health, age- related changes in pharmacodynamics and pharmacokinetics, co-occurrence of multiple disease conditions, and polypharmacy. Consequently, the unnecessary high prescribing of potentially inappropriate medications (PIMs) in older adults is frequently documented in different settings of care. The aim of our study was to compare the prevalence of PIM use in older adults in acute care, ambulatory care and community pharmacy practices in the Czech Republic.

Method: A cross-sectional multicentric study was conducted in several regions of the Czech Republic as part of the EuroAgeism H2020 ESR7 project. We included older adults aged 65+ years without serious cognitive impairment, serious problems of hearing and speaking, and not in end-of-life or intensive care. Data were collected prospectively using a structured protocol based on comprehensive geriatric assessment. The source of data were medical records, interviews with patients and healthcare professionals, and clinical assessments. We assessed the prevalence of PIM use by explicit criteria - EU(7)-PIM list and American Geriatrics Society 2019 Updated Beers Criteria. Stepwise multiple logistic regression was used to identify potential risk and protective factors of PIM use. Main outcome measures: Prevalence and protective/risk factors of PIM use.

Results: We assessed 1602 patients (589 acute care, 563 ambulatory care, 450 community pharmacy practices). In the total sample, $66 \%$ of older adults were females, $69.3 \%$ used 5 and more medications (polypharmacy), and $62.8 \%$ had 4 and more chronic diseases (polymorbidity). Overall, Beers 2019 criteria identified PIM use in $18.5 \%$ patients, EU(7)-PIM list in $68.4 \%$ patients, and both criteria in $68.5 \%$. The total PIM prevalence was $37.1 \%$ in community pharmacy practices, $74.6 \%$ in ambulatory care and $86.8 \%$ in acute care. The risk of PIM use was significantly lower $(p<0.05)$ in the ambulatory care setting and community pharmacy practices $(0.6$ $(0.4-0.8)$ and $0.4(0.2-0.5)$, respectively) than in acute care. Higher risks of PIM prescribing was confirmed for older adults aged $75+$ years (1.6 (1.2-2.2)); taking 5+ medications (8.4 (6.2-11.2)); and being diagnosed with depression (1.7 (1.1-2.6)).

Conclusion: In the Czech sample of older adults participating in the EuroAgeism project, the overall prevalence of PIM use was confirmed to be the highest in acute care and lower in ambulatory care and community pharmacy practices. Polypharmacy and depression were found to be important modifiable correlates of PIM use in older adults.

Grants: EuroAgeism H2020 MSCF-ITN-764632, Inomed NO.CZ.02.1.01/0.0/0.0/18_069/0010046, Progress Q42- Faculty of Pharmacy, Charles University, START/MED/093CZ.02.2.69/0.0/0.0/ 19_073/0016935, SVV 260551 and ICARE4OLD H2020 -965341.

\section{PDF01.3}

Analysis of the impact of education on adolescents' knowledge about medicines

\section{Jana Urbankova ${ }^{*}$, Magdalena Kuzelova ${ }^{1}$}

${ }^{1}$ Department of Pharmacology and Toxicology, Faculty of Pharmacy, Comenius University in Bratislava, Bratislava, Slovakia

Background and Objective: Adolescents often take medicine independently without supervision of an adult despite having little knowledge about the proper use of medicines. However, there is no systematic education for children and adolescents in the Slovak Republic regarding this topic. The aim of this study was to analyze the effect of education on attitudes of Slovak adolescents towards efficacy and safety of medicines and on their knowledge about medicines. 
Method: Results were processed from self-reported online questionnaires prepared and validated in our previous study (Klimaszova et al., 2016). A total number of 122 adolescents aged 15-18 years from 4 different schools in Bratislava, Slovak Republic filled out the same questionnaire before and after the education. The effect of the education was evaluated using paired Student's t-test comparing the mean values from answers to questions about knowledge and attitudes towards medicine expressed as items of the 5-degree Likert scale.

Main outcome measures: To identify differences in knowledge about medicines before and after the education of adolescents.

Results: Most of the adolescents rated their overall health as good $(45.1 \%)$ or very good $(40.2 \%) .42 .6 \%$ of the adolescents suffered from a chronical illness, most frequently allergies (26.2\%). In past 6 months, $89.4 \%$ of the adolescents took OTC medicine and $65.0 \%$ of the adolescents took it without parental supervision. In addition, $42.6 \%$ of the adolescents had taken prescription medicine in past 6 months, mostly antihistamines for systemic use (35.1\%). The majority $(66.4 \%)$ stated, that they talk about medicines with their parents and $76.2 \%$ marked that when they feel sick, they immediately inform their parents, who help them resolve the situation. After the education, there was a significant improvement in every question regarding adolescents' knowledge on medicines. Adolescents mostly improved their knowledge in questions about the risk of allergy medicines, painkillers, cough medicines, long-term use nasal drops and antibiotics $(\mathrm{p}<0.001)$. Smaller, but still significant difference, was in answers to the questions about the danger of combining alcohol with medicine $(p=0.001)$ and about the possibility of an adverse effect when more than recommended dose of medicines is taken $(\mathrm{p}=0.002)$. These two topics were well known by most of the students already before the education. There was also a significant improvement in adolescents' belief about the efficacy of both prescription and OTC medicines and about the safety of prescription medicines $(\mathrm{p}<0.001)$. Conclusion: Education is a needed and efficient tool for eliminating lack of basic knowledge regarding the use of medicines. Therefore, it is important to educate children and adolescents as well as their parents about the responsible use of medicines.

References: KLIMASZOVA Z, FAZEKAS T, KUZELOVA M. 2016. Development and validation of novel self-report questionnaire about the assessment of adolescents' relation to medicines use and risk. Eur Pharmaceut J. 2016, 63, 1, 3-35.

\section{PDF01.4}

Young people and the management of chronic illness by primary care pharmacists

\section{Mohammed Almunef* ${ }^{1}$, Julie Mason ${ }^{1}$, Chris Curtis ${ }^{1}$, Zahraa Jalal $^{1}$}

${ }^{1}$ College of Medical and Dental Sciences, University of Birmingham, Birmingham, United Kingdom

Background and Objective: Recent evidence has shown that the incidence of long-term illnesses in young people is increasing ${ }^{(1)}$. Pharmacists, as medicine experts, are in a unique position to promote young people's health. The aim of this study was to explore the role of primary care pharmacists in the management of chronic illnesses in young people aged 18-24 years.

Method: A qualitative study was undertaken. 22 primary care pharmacists in the UK were recruited through purposeful sampling. Semistructured interviews were conducted and audio recorded, transcribed verbatim and analysed using thematic analysis. Ethical approval was obtained from the University of Birmingham Ethics Committee.

Main outcome measures: The main focus was on primary care pharmacists' roles in caring of young people with chronic illness.
Pharmacists' perceptions about young people medication-related experiences, and views on pharmaceutical care services provided to young people and suggestions for improvement was also explored.

Results: Participants identified several roles for primary care pharmacists in caring of young people with chronic illness. These roles included encouraging young people to visit the pharmacy to collect their medicines and ensuring that they have enough medicines supply, maintaining young people medication safety, counselling and educating young people about their medicines and answering their queries, building trusted relationships directly with them, provision of specialist services, following up with young people and checking on medication compliance, and signposting them for further support.

Conclusion: Primary care pharmacists feel that they have an important role in supporting young people with chronic illness. This study identified many ways in which pharmacists provide services and support to young people. Future research is necessary to provide more evidence of the benefit of primary care pharmacists in supporting young people with chronic illness in the optimal use of their medication.

References: 1. International Comparisons of Health and Wellbeing in Adolescence and Early Adulthood. Available online: https:// www.nuffieldtrust.org.uk/research/international-comparisons-ofhealth-and-wellbeing-in-adolescence-and-early-adulthood (accessed on 3 June 2021).

\section{PDF01.5}

\section{Effectiveness of tocilizumab in COVID-19 infection}

Javier Romero Puerto ${ }^{1}$, Alberto Soria Martin², Javier Garcia Marin $^{*}$, Adriana Moreno Herrera ${ }^{2}$, Marina Fages Perez ${ }^{2}$

\section{${ }^{1}$ FARMACIA, HOSPITAL NAVALMORAL DE LA MATA, CACERES, ${ }^{2}$ FARMACIA, HOSPITAL PUNTA DE EUROPA, ALGECIRAS, Spain}

Background and Objective: To evaluate the efficacy of tocilizumab in hospitalized patients with Coronavirus SARS-COV-2 (Covid-19) infection.

Design: Descriptive retrospective study. All patients admitted with Covid-19 infection treated with Tocilizumab in March and April 2020, and who had resolved their clinical situation at the date of the study, were included. Data were obtained from the Athos-Prisma ${ }^{\circledR}$ prescription program, the Diraya ${ }^{\circledR}$ medical record review, and the analytics program. The following variables were collected: sex, age, previous treatments, D-dimer and C-reactive protein (CRP) values before and after Tocilizumab administration, time from symptom onset to administration and outcome (death / discharge). The Tocilizumab regimen used was $600 \mathrm{mg}$ single dose. All patients were previously treated with hydroxychloroquine 200 milligrams $(\mathrm{mg})$ every 12 hours (h) $(400 \mathrm{mg} / 12 \mathrm{~h}$ on the first day) for 5 days, concomitant with lopinavir $400 \mathrm{mg} /$ ritonavir $100 \mathrm{mg}$ every $12 \mathrm{~h}$ for 14 days. Concomitant treatments to the previous regimen of hydroxychloroquine and lopinavir/ritonavir were used in 7 patients: 6 patients were treated concomitantly with azithromycin for 3 days and one patient was treated with interferon $1 \mathrm{~b} 0.25 \mathrm{mg}$ subcutaneous every 48 $\mathrm{h}$ for 8 days.

Results: 14 patients were included, 11 women and 3 men, with a mean age of 65.4 (53.3-76.7) years, of which 7 died and 7 were discharged from hospital during the study period. The mean hospitalization period was 25.4 (13.3-37.53) days. The mean time elapsed from the onset of symptoms to the administration of Tocilizumab was 14 (8.1-19.9) days in the group of deaths and 11.2 (8.39-14.01) days for the group The mean plasma $\mathrm{D}$-dimer concentration prior to Tocilizumab administration was $2989 \mathrm{ng} / \mathrm{ml}$ and a post-administration 
value of $10789 \mathrm{ng} / \mathrm{ml}$. The mean C-reactive protein (CRP) value prior to Tocilizumab administration was $133 \mathrm{mg} / \mathrm{L}$ and a post-administration value of $100 \mathrm{mg} / \mathrm{L}$.

Conclusion: In our study, the administration of Tocilizumab reduced the CRP values but did not manage to reduce plasma concentrations of D-dimer. The time elapsed until the administration of Tocilizumab was highly variable within each subgroup (death/discharge), with which it could have no clinical relevance. Our small sample size does not allow general conclusions to be drawn for the rest of the population, so a greater number of studies are necessary

\section{PDF01.6}

\section{COVID-19 vaccination: a new window for clinical pharmacy}

Ana Rebelo ${ }^{1}$, Ana Fernandes ${ }^{1}$, Cristiana $\operatorname{Ramos}^{1}$, Inês Prata ${ }^{1}$, Marcos Ferreira $^{1}$, Miguel Dias" ${ }^{*}$, Patrícia Pereirinha ${ }^{1}$, José Feio $^{1}$, Patrícia Paiva ${ }^{2}$, Francisco Parente ${ }^{2}$

${ }^{1}$ Pharmacy, ${ }^{2}$ Clinical Pharmacology, Coimbra Hospital and University Centre, Portugal, Coimbra, Portugal

Background and Objective: During the Covid-19 pandemic, hospital pharmacists were essential in the process of optimizing vaccination from clinical, technical to logistical areas.

Taking into account that the covid-19 vaccine is a medicine subject to additional monitoring, we intend to understand the impacts caused by it, promoting an active pharmacovigilance.

Design: In Coimbra Hospital and University Centre, a multidisciplinary network was created to define all the steps and stakeholders in the vaccination task of the healthcare professionals of the hospital. To achieve greater guarantee of quality, pharmacists were placed at each stage of the circuit, including the creation of a satellite pharmacy next to the vaccination posts.

The pharmacist in the satellite pharmacy as part of a multidisciplinary team adresses to safety, quality, and scientific issues in order to ensure the responsible use of the medicine. In this context, the pharmacist clarifies possible interactions, monitors side effects, reports adverse drug reactions and promotes rational use. An ongoing research study was developed by the multidisciplinary group that aims to collect information of high clinical value to promote active pharmacovigilance. To collect and manage the information we used Google Forms, clinical software (Sistema de Gestão Integrado do Circuito do Medicamento, SClínico) and Microsoft Office Excel as software tools.

Results: A query was sent to the 4369 healthcare professionals vaccinated with 2 doses of Covid-19 mRNA vaccine (nucleosidemodified) during the period from December 2020 to February 2021, resulting in a total of 2909 answers (2186 female and 723 male). Regarding the first dose, 1689 very common adverse reactions were recorded, 354 common and 173 uncommon and in the second dose 1999, 440 and 548 respectively.

At the satellite pharmacy, the pharmacist along the immunoallergology team dispensed 57 antihistamines to prevent adverse effects and dispensed to the emergency room several medications such as corticosteroids, antihypertensive and benzodiazepines to prevent and control the negative outcomes.

Conclusion: The constant presence of the pharmacist in this vaccination process reinforced the importance of training and continuous integration of clinical pharmacy in multidisciplinary teams, allowing traceability, safety and efficiency, always ensuring the quality of the process.

\section{PDF01.7}

The impact of a simulated continuing professional development course on maintaining pharmacy students' mental health and well-being during COVID-19

\section{Sarah Khamis $^{*}$, Hebah Sallom ${ }^{1}$, Bilgen Basgut ${ }^{2}$}

${ }^{1}$ Clinical Pharmacy, Near East University, Mersin 10, ${ }^{2}$ Faculty of pharmacy, department of pharmacology, Baskent University, Ankara, Turkey

Background and Objective: The impact of COVID-19 pandemic on the mental health of both current and future healthcare providers is detrimental. Dramatic changes in pharmacy students' day-to-day lives led their psychological health to become a major concern necessitating effective interventions. To examine the effectiveness and utility of a continuing professional development (CPD) training course in improving students' competence in maintaining mental health and well-being during the current COVID-19 pandemic and similar crisis, along improving their lifelong learning (LLL) skills.

Design: A CPD simulation course was introduced to a cohort of fifth year students in Northern Cyprus. The course was delivered as an interactive orientation course, with a follow up of the impact of the course on students during their internship. A pre-post validated questionnaire and scales were utilized to evaluate outcomes of the course. Kessler-10 (K10) was adopted to measure psychological distress.

Results: 75 fifth-year students were invited to fill the questionnaire, of which $37 \%$ responded. Respondents were divided into randomly into intervention and control group. Of participants, 58\% were female. Self-reported change in mental well-being since COVID-19 onset, show worsening of well-being as reported by $46 \%$ of students while $38 \%$ reported no change. All participants in both group of students show mental distress on baseline and post assessment. The mean K10 score pre and post the course in the control group were $(22.64 \pm 8.5 ; 25.07 \pm 10)$ and in the intervention group were $(25.90$ $\pm 4.9 ; 27.1 \pm 9.3$ ) indicating moderate psychological distress with no significant differences. Students' in intervention group show significant improvement in their awareness about self-directed LLL skills, and mental health challenges and prompting services compared to control and baseline assessment.

Conclusion: The CPD simulation course provided students with opportunities to enhance their LLL skills and maintaining mental well-being both essential during COVID19 and similar crisis, yet improvement in mental distress was not significant compared to control.

\section{PDF01.8}

Impact of polypharmacy on frequency of falls and hospitalizations in older adults: Croatian study from the EUROAGEISM H2020 project

\section{Margita Držaić ${ }^{1,2}$, Ingrid Kummer* 2, 3, Iva Bužančić ${ }^{1,2}$, Maja Marušić $^{4}$, Martina Šepetavc ${ }^{5}$, Andrea Bošković ${ }^{1}$, Jovana Brkić ${ }^{3}$, Maja Ortner Hadžiabdić ${ }^{1}$, Daniela Fialová ${ }^{3,6}$}

${ }^{1}$ Faculty of Pharmacy and Biochemistry, University of Zagreb, ${ }^{2}$ City Pharmacies Zagreb, Zagreb, Croatia, ${ }^{3}$ Department of Social and Clinical Pharmacy, Faculty of Pharmacy in Hradec Králové, Charles University, Hradec Králové, Czech Republic, ${ }^{4}$ Community Pharmacy Sonja Grbac Stublić, ${ }^{5}$ Farmacia community pharmacy chain, Zagreb, Croatia, ${ }^{6}$ Department of Geriatrics and Gerontology, 1st Faculty of Medicine, Charles University in Prague, Prague, Czech Republic 
Background and Objective: The aim of this study was to explore the association between number of prescribed medications and their impact on frequency of falls and hospitalizations in community-residing older adults.

Method: This observational, cross-sectional study was conducted as part of the EUROAGEISM H2020 ESR 7 project. Presented data are preliminary and include 84 adults aged $\geq 65$ years who attended three community pharmacies in the City of Zagreb. Data were collected using structured, standardized questionnaire developed for the purpose of the EUROAGEISM H2020 project. Descriptive and inferential statistical methods were applied to analyse data using IBM SPSS v 20.

Main outcome measures: Association between number of prescribed medications and the incidence of falls and hospitalizations in the last 12 months.

Results: Final analysis included 84 participants (67.9\% female; median age 73 (IQR 68-80)). Every participant used on average $5,62 \pm 2,916$ prescribed medications, while polypharmacy $(5+$ medications) was identified in $51(60,7 \%)$ of them. Use of at least one medication from the benzodiazepine drug class was observed in 32 $(38,1 \%)$ of the participants, with females using them statistically significantly more often than males $\left(X^{2}(1)=4,251, \mathrm{p}<0,05\right)$. Of the total number of participants, $57(67,9 \%)$ experienced a fall in the past, of which $17(20,2 \%)$ in the last 12 months. Female participants have fallen statistically significantly more often in the past than males $\left(X^{2}(1)=4,673, \mathrm{p}<0,05\right)$. Only $12(14.3 \%)$ participants were hospitalized in the last 12 months, while men were statistically significantly more often hospitalized than women $\left(X^{2}(1)=4,403, \mathrm{p}<0,05\right)$. A positive trend was observed showing that higher number of medications were prescribed in participants who fell $(6.00 \pm 2.716$ vs. 5.52 $\pm 2.976 ; \mathrm{p}>0.05)$ or were hospitalized $(6.58 \pm 2.937$ vs. $5.46 \pm$ $2,902 ; \mathrm{p}>0.05)$ in the last 12 months, however, the statistical significance was not confirmed.

Conclusion: This study provides preliminary results regarding association between number of medications prescribed and frequency of falls and hospitalizations in community-residing older adults in the City of Zagreb and indicates the importance of pharmacotherapy optimisation in this vulnerable age group.

Grant support: EuroAgeism H2020 MSCF-ITN-764632, Inomed NO.CZ.02.1.01/0.0/0.0/18_069/0010046, Progress Q42- Faculty of Pharmacy, Charles University, START/MED/093 CZ.02.2.69/0.0/0.0/ 19_073/0016935, SVV 260551 and ICARE4OLD H2020 -96534.

\section{PDF01.9}

Use of fall-risk-increasing drugs in older patients admitted to the department of emergency medicine- a retrospective study with focus on central nervous system drugs

\section{Liesbeth Huys ${ }^{*}$, Peter De Paepe ${ }^{2}$, Mirko Petrovic ${ }^{3}$, Andreas Capiau $^{1,4}$, Sabrina Commeyne ${ }^{1}$, Annemie Somers ${ }^{1,} 4$ \\ ${ }^{1}$ Department of Pharmacy, ${ }^{2}$ Department of Emergency Medicine, ${ }^{3}$ Department of Geriatrics, Ghent University Hospital , \\ ${ }^{4}$ Pharmaceutical Care Unit, Ghent University, Ghent, Belgium}

Background and Objective: Falls and fall-related injuries are an increasing problem, primarily in older people. Certain drug classes are associated with an increased risk of falls and are therefore called fall-risk-increasing drugs (FRIDs).

We aimed (i) to evaluate the use of FRIDs, sedative and anticholinergic drugs as well as drugs with a risk of orthostatic hypotension; (ii) to determine the incidence of falls and the characteristics of patients with and without falls; (iii) and to analyse potentially inappropriate prescribing (PIP) with focus on drugs that act on the central nervous system (CNS) in a population of older patients.
Method: A retrospective study of older patients ( $\geq 65$ years) admitted to the department of emergency medicine of Ghent University Hospital between October 2020 and January 2021, in whom a medication reconciliation was performed by a hospital pharmacist.

Main outcome measures: Number of prescribed FRIDs, sedative and anticholinergic drugs and drugs with risk of orthostatic hypotension (OH); Sedative Load Model (SLM) and Anticholinergic Impregnation Scale (AIS) score of drugs; incidence of falls; CNS PIP using the STOP-NL criteria.

Results: For 200 patients 1791 drugs, of wich 596 FRIDs were identified (median 3, IQR 1-4). A total of $32.9 \%$ were CNS drugs, with opioids and hypno-sedatives being the most frequently prescribed classes of FRIDs. There was a positive association between the number of FRIDs and the total number of drugs, the number of comorbidities, the number of sedative and anticholinergic drugs as well as the number of $\mathrm{OH}$ inducing drugs, the SLM and AIS score ( $\mathrm{p}$ $<0.001)$. Fifty patients $(25 \%)$ reported a fall in the recent or past history. Furthermore, age $(p=0.014)$, sex $(p<0.001)$ and rate of PIP $(p<0.001)$ were significantly different between patients with and without falls. Almost one-fourth of patients was treated with at least one CNS PIP item.

Conclusion: The prevalence of FRID use was high in older patients admitted to the department of emergency medicine of Ghent University Hospital. Fallers had a higher number of CNS PIP items. The results from this study confirm the need for multidisciplinary medication review, with focus on attempts for deprescribing of CNS FRIDs.

\section{POSTER DISCUSSION FORM II}

\section{PDF02.1}

Mental health hospital in the home clinical pharmacist improves medicines safety

\section{Mechaiel Farag ${ }^{*}{ }^{1,}$, , Leanne Chalmers ${ }^{1}$, Kreshnik Hoti ${ }^{1,3}$, Jeff Hughes $^{1}$}

${ }^{1}$ Curtin Medical School, Curtin University, Bentley, ${ }^{2}$ North Metropolitan Health Service - Mental Health Pharmacy, Graylands Hospital, Mount Claremont, Australia, ${ }^{3}$ Faulty of Medicine, University of Prishtina, Prishtina, Kosovo, -

Background and Objective: Mental Health HiTH (MH-HiTH) services aim to prevent hospital admissions or facilitate early discharge. Integration of a clinical pharmacist into multidisciplinary MH-HiTH teams is becoming more common but little is known about the benefits of their involvement. This study evaluated whether clinical pharmacist involvement in $\mathrm{MH}-\mathrm{HiTH}$ improved medication safety.

Method: In a retrospective cohort study, medical records were reviewed of all patients admitted to two MH-HiTH services between 1 September and 30 November 2015. The first site (Site 1) is a 16-bed $\mathrm{MH}-\mathrm{HiTH}$ service based in a tertiary psychiatric hospital incorporating a clinical pharmacist as part of its multi-disciplinary team. The second site (Site 2) is an 18-bed MH-HiTH service based in a tertiary general hospital without clinical pharmacist involvement. Completion of medication management activities was compared using chi-square analysis.

Main outcome measures: Five measures based on the WA Health Pharmaceutical Review Policy: (1) medication reconciliation on admission and discharge; (2) accurate adverse drug reaction (ADR) list; (3) documentation of a current medication profile during admission and on discharge; (4) provision of patient medication information; and (5) regular medication profile review. 
Results: Seventy-five patient records from Site 1 were assessed and 59 from Site 2. Patient characteristics from both sites were comparable in terms of age, sex, diagnosis and source of admission. The HiTH service incorporating a pharmacist (Site 1) demonstrated statistically significantly higher rates of completion of medication reconciliation (87\% versus 29\%), accurate ADR list (97\% versus $58 \%$ ), accurate discharge medication list ( $74 \%$ versus $45 \%$ ), accurate medication profile $(99 \%$ versus $68 \%)$ and medication chart review (99\% versus $0 \%$ ); all $\mathrm{p}<0.001$.

Conclusion: Integrating a clinical pharmacist into a MH-HiTH program significantly improved medication safety parameters, and has the potential to reduce adverse outcomes.

\section{PDF02.2}

Optimising physical health treatment in mental illness that is severe (OPTIMISE)

\section{Aoife Carolan $^{*}$ 1, 2 , Dolores Keating ${ }^{1}$, Caroline Hynes ${ }^{1}$, Stephen McWilliams $^{3}$, Judith Strawbridge ${ }^{4}$, Cristin Ryan ${ }^{5}$}

${ }^{1}$ Pharmacy Department, SAINT JOHN OF GOD HOSPITAL, ${ }^{2}$ Royal College of Surgeons Ireland, ${ }^{3}$ SAINT JOHN OF GOD HOSPITAL, ${ }^{4}$ School of Pharmacy and Biomolecular Science, Royal College of Surgeons Ireland, ${ }^{5}$ School of Pharmacy and Pharmaceutical Sciences, Trinity College Dublin, Dublin, Ireland

Background and Objective: The life expectancy of people with Severe Mental Illness (SMI) is shorter than those without SMI, with multimorbidity and poorer physical health contributing to health inequality. Screening tools could potentially assist the optimisation of medicines to protect the physical health of people with SMI. We designed a medicines optimisation tool (OPTMISE) to help clinicians achieve the goal of Optimising Physical Health in Mental Illness that is Severe.

Method: OPTMISE was drafted with reference to the literature, and the Delphi consensus technique used to develop and validate the contents. A 17-member multidisciplinary panel of experts from the UK and Ireland completed 2 rounds of Delphi consensus, rating their level of agreement to 83 prescribing indicators using a 5-point Likert scale. Indicators with a median of 1 or 2 and $75^{\text {th }}$ centile value of $\leq 2$ were accepted. Interrater reliability was assessed among 4 clinicians across 20 datasets and the chance corrected level of agreement (kappa) was calculated.

Main outcome measures: Level of agreement using a 5-point likert scale and interrater reliability were the main outcome measures assessed.

Results: Consensus was achieved after 2 rounds of Delphi for 63 prescribing indicators. Interrater reliability of OPTIMISE between physicians and pharmacists indicated a substantial level of agreement, which is comparable to other optimisation tools.

Conclusion: OPTIMISE is a 63 indicator medicines optimisation tool, developed using Delphi consensus techniques, to assist decision making in treating people with SMI. The tool has the potential to enhance medicines optimisation, ensuring preventative medicines are considered when clinically indicated. Further robust research studies involving the implementation of this prescribing tool is required to demonstrate its true benefit.

\section{PDF02.3}

Key factors underlying oncology patients' willingness to engage in medication redispensing

Lisa-Marie Smale $^{* 1}$, Toine Egberts ${ }^{2,3}$, Rob Heerdink ${ }^{3,}$, Bart van den Bemt ${ }^{5,6}$, Charlotte Bekker

${ }^{1}$ Department of Clinical Pharmacy, Radboud Institute for Health Sciences, Radboud university medical center, Nijmegen, ${ }^{2}$ Clinical Pharmacy, University Medical Centre Utrecht, Department of Clinical Pharmacy, Division of Laboratory and Pharmacy, ${ }^{3}$ Division of Pharmacoepidemiology and Clinical Pharmacology, Utrecht University, Faculty of Science, Utrecht Institute for Pharmaceutical Sciences (UIPS), ${ }^{4}$ Research Group Innovations of Pharmaceutical Care, Utrecht University of Applied Sciences, Utrecht, ${ }^{5}$ Department of Clinical Pharmacy, Radboud university medical center, Radboud Institute for Health Sciences, ${ }^{6}$ Department of Pharmacy, Sint Maartenskliniek, Nijmegen, Netherlands

Background and Objective: Redispensing medication unused by patients could potentially reduce the financial loss and environmental pollution caused by medication waste, particularly for expensive medication such as oral anticancer drugs. To achieve medication redispensing patients' engagement is required. Therefore this study aims to identify key factors underlying oncology patients' willingness to engage in the redispensing of unused oral anticancer drugs.

Method: Semi-structured interviews via telephone or video call were conducted with adult oncology patients from two Dutch hospitals. The interview guide was framed using the COM-B model, to elicit patients' capability, opportunity and motivation to engage in medication redispensing. Questions related to patients' willingness to accept redispensed medication, reasons thereof, and perceived concerns and needs. Inductive thematic analysis was applied, and emerging key factors were mapped to the COM-B model.

Main outcome measures: Key factors underlying willingness of oncology patients to engage in medication redispensing mapped to the COM-B model.

Results: Seventeen patients (aged 38-82 years, 70\% female), suffering eight different types of cancer participated. The majority of participants supported medication redispensing.

Four categories of key factors underlying oncology patients' willingness to engage in medication redispensing were identified. First, perception of social relevance was identified as the driver for engaging in medication redispensing. This perception resulted from previous frustrations with medication waste and social responsibility regarding the healthcare budget and environment. The second key factor was trust in quality, influenced by the initial perception of the quality of redispensed medication, knowledge about the quality process and advocacy by caregivers and relatives. Finally, two facilitators for engaging in medication redispensing were identified: convenient logistics, which related to return possibilities of unused medication to pharmacies, and transparent communication, about quality control, financial profits and the consequences of medication waste. All factors were mapped to the COM-B model of behavioural change.

Conclusion: This study shows that oncology patients want to engage in medication redispensing with a drive for achieving positive societal impact and a need for high-quality medication, transparent communication and a convenient process as underlying key factors. Future interventions that increase oncology patients' capability, opportunity and motivation may support willingness to engage in medication redispensing. 


\section{PDF02.4}

Polypharmacy and medication nonadherence as modifiable correlates of self-reported poorer health status in communityresiding older patients

Ingrid Kummer $^{* 1,2}$, Margita Držaić ${ }^{1}$, Maja Ortner Hadžiabdić ${ }^{3}$, Iva Bužančić ${ }^{1}$, Maja Marušić ${ }^{4}$, Danijela Butković ${ }^{5}$, Marko Kolarić $^{6}$, Hrvoje Jurinić ${ }^{7}$, Marina Matković ${ }^{8}$, Lea Brečević ${ }^{3}$, Tea Buršić ${ }^{3}$, Andrea Bošković ${ }^{3}$, Jovana Brkić ${ }^{2}$, Daniela Fialová ${ }^{2}$,

${ }^{1}$ Zagreb City Pharmacies, Zagreb, Croatia, ${ }^{2}$ Social and Clinical Pharmacy, Faculty of Pharmacy in Hradec Králové, Czech Republic, Hradec Králové, Czech Republic, ${ }^{3}$ Faculty of Pharmacy and Biochemistry, ${ }^{4}$ Community Pharmacy Sonja Grbac Stublić, Zagreb, ${ }^{5}$ Community Pharmacy chain Lijek Đakovo, Đakovo, ${ }^{6}$ Community Pharmacy Poljak-Radnić, Sibinj, ${ }^{7}$ Community Pharmacy Šibalić, Vinkovci, ${ }^{8}$ Community Pharmacy chain Ljekarna Pablo, Rijeka, Croatia, ${ }^{9}$ Department of Geriatrics and Gerontology, 1st Faculty of Medicine, Charles University, Prague, Czech Republic

Background and Objective: Self-reported health status is shown to be a potent indicator of patients' overall health prognosis and some health-related outcomes. The aim of our study was to explore various risk factors (sociodemographic, clinical and medication-related) that significantly correlate with self-reported poor health status in community-residing older patients in Croatia.

Method: 210 patients aged 65 years and older attending community pharmacies in 3 different regions of Croatia (Zagreb, Istria and Slavonia) were prospectively assessed using method of comprehensive geriatric assessment with the EUROAGEISM H2020 ESR7 study protocols (Jun2019-Jan2020). Descriptive and inferential statistical methods were applied to analyse data by the SPSS statistical program vers. 20.

Main outcome measures: Correlation between older patients' selfreported poor health and sociodemographic, clinical and medicationrelated risk factors was tested.

Results: The mean age of study participants was $74.7 \pm 6.6$ years and the majority were female $(64.8 \%)$. $50.5 \%$ patients reported their health status to be good or very good, $39.0 \%$ as moderate and $10.5 \%$ as poor or very poor. Male patients and patients 80 years and older perceived their health to be poorer $(r=-0.231, p=0.001 ; r=-0.182$, $\mathrm{p}=0.009$ ), as well as patients using polypharmacy ( $\geq 5$ medications, $61.0 \%)(\mathrm{r}=-0.162, \mathrm{p}=0.019)$. Statistically significant correlation was found also between self-reported poorer health and nonadherence to medication therapy $(\mathrm{r}=0.149, \mathrm{p}=0.031)$.

Conclusion: Self-reported poorer health in community-residing older adults in our study correlated with some unaffected risk factors as male gender and higher age, but also with modifiable correlates as polypharmacy and nonadherence to medication therapy. The last two risk factors might be positively influenced by early clinical pharmacy interventions and pharmacotherapy optimisation.

Grants: EuroAgeism Horizon 2020 MSCF-ITN-764632, Inomed NO.CZ.02.1.01/0.0/0.0/18_069/0010046, Progress Q42- Faculty of Pharmacy, Charles University, START/MED/093 CZ.02.2.69/0.0/0.0/ 19_073/0016935, SVV260 551 and I-CARE4OLD H2020 -965341

\section{PDF02.5}

Workflow and time requirement of medication documentation on paper or with a computerized physician order entry (CPOE) system

Viktoria Jungreithmayr $^{* 1,}$, , Elisabeth K. Rein ${ }^{1,2}$, Walter E. Haefeli $^{1,2}$, Hanna M. Seidling ${ }^{1,2}$ on behalf of Implementation Team (members: C. Benkert, J. Bittmann, M. Fabian, A. Geisbüsch, S. Hagmann, M. Innmann, U. Klein, S. Klingebiel, C. Kobus-Schubach, S. Kugler, M. Löpprich, C. Merle, M. Österreicher, O. Reinhard, L. Scholz, B. Zeeh)

${ }^{1}$ Department of Clinical Pharmacology and Pharmacoepidemiology, ${ }^{2}$ Cooperation Unit Clinical Pharmacy, University Hospital Heidelberg, Heidelberg, Germany

Background and Objective: Implementing a CPOE system changes workflows and redistributes tasks among health care professionals. Particularly physicians reported spending more time documenting medication after the implementation of a CPOE system [1]. This study aims to exemplarily describe workflow changes and objectify the time required for medication documentation on paper and with a CPOE system (Cerner ${ }^{\circledR}$ i.s.h.med).

Method: In a first step, the prescription process was evaluated in semi-structured interviews with the head nurses and senior physicians of three orthopaedic university hospital wards. Two case scenarios were then developed, consisting of virtual patients with exemplary medication (case 1: $n=6$ drugs; case 2: $n=11$ drugs). Six resident physicians and six nurses of the respective wards each formed a pair and were asked to perform the medication documentation of the case scenarios according to the workflows established before and after CPOE implementation. The time needed to document the exemplary medication orders was measured using the time-tracking app Toggl Track ${ }^{\circledR}$. Time durations were then compared between the different workflows and separately for each healthcare profession (MannWhitney-U-Test).

Main outcome measures: Time difference in medication documentation following the paper-based process or the CPOE-based process. Results: The process evaluation of the three considered wards revealed that prior to CPOE implementation, both physicians and nurses were involved in the medication documentation, the process consisted of eight workflow steps, and two documentation systems (paper charts and an electronic system) where medication was documented twice were used, whereas afterwards medication documentation was solely a physicians' task, consisted of five workflow steps, and one documentation system (the CPOE system) was used. The median time needed for medication documentation on paper was 04:21 $\mathrm{min}$ (range: 03:11-07:03 $\mathrm{min}$ ) and 03:59 $\mathrm{min}(03: 10$ 05:47) with the CPOE system for case 1 and 07:20 min (04:18-11:24) and 08:18 $\min (06: 23-10: 05)$ for case 2, respectively. Overall, the time needed for medication documentation of both cases did not differ (median=05:25 min on paper and 06:05 min with the CPOE system, $\mathrm{p}=0.799)$. The median time difference was 00:11 $\min (-03: 39-01: 24$ $\min , \mathrm{p}=0.818)$ for case 1 and 00:05 $\min (-01: 32-04: 35 \mathrm{~min}, \mathrm{p}=0.485)$ for case 2. Physicians took a median of 02:45 min (00:06-07:31 min, $\mathrm{p}=0.001$ ) longer per case to document medication in the CPOE system than on paper. Nurses had a median time saving of 02:30 min (-01:18 min to $-04: 51 \mathrm{~min}, \mathrm{p}<0.001)$ per case after CPOE implementation. Conclusion: This study revealed that on the three wards, the overall time difference for medication documentation on paper or with the CPOE system was small. However, due to changes in task responsibility and the workflow, physicians' time did increase with CPOE implementation, while nurses experienced time savings. For a future overall assessment, it is important to put these results in relation to the quality of medication documentation. 
References: 1. Niazkhani Z, Pirnejad H, Berg M, Aarts J. The impact of computerized provider order entry systems on inpatient clinical workflow: a literature review. Journal of the American Medical Informatics Association : JAMIA. 2009;16(4):539-49.

\section{PDF02.6}

Influence of baricitinib and tofacitinib on cholesterol levels

Alberto Soria Martin ${ }^{1}$, Javier Romero Puerto ${ }^{2}$, Sol P. Cortes de Miguel $^{1}$, Marina Fages Perez ${ }^{1}$, Javier Garcia Marin ${ }^{*} 1$

${ }^{1}$ FARMACIA, HOSPITAL PUNTA DE EUROPA, ALGECIRAS, ${ }^{2}$ HOSPITAL NAVALMORAL DE LA MATA, CACERES, Spain

Background and Objective: Baricitinib and Tofacitinib are inhibitors of JAK kinases (iJAK) used mainly in rheumatoid arthritis. iJAK can increase cholesterol levels.

The objectiv is to assess the increase in total cholesterol $(\mathrm{Ct})$ in patients with iJAK

Design: Retrospective observational study of patients on treatment with IJAK between February 2018 to April 2020. The data were obtained from the PRISMA-APD $\AA$ outpatient care program, and by reviewing medical records in Diraya ${ }^{\circledR}$. The variables collected were: age, sex, adherence, start and end date of treatment, dose reduction, baseline total cholesterol $(\mathrm{Cb}), \mathrm{Ct}$ during the study and previous lipidlowering treatments or their new prescription. The data were analyzed using the SPSS ${ }^{\circledR}$ statistical program, using the Student's t test for paired data.

Results: 31 patients were treated: 12 were excluded, 7 for lack of analytical parameters and 5 for adherence less than $85 \%$. Finally, 19 patients were included, 16 women and 3 men. The median age was 52 years (19-74).

The $\mathrm{Cb}$ of 10 patients was below $200 \mathrm{mg} / \mathrm{dL}$. In 6 patients, $\mathrm{Ct}$ increased above $200 \mathrm{mg} / \mathrm{dL}$ (hypercholesterolemia), with a median onset of hypercholesterolemia after starting iJAK of 6.57 weeks (1.71-10.29). 3 patients remained statin-free, 2 continued with the same statin (it was necessary to reduce the baricitinib dose due to dyslipidemia in one case), and 1 patient required the prescription of a statin.

The median duration of iJAK treatment was 9.77 months (1.90-16.90). The median until the first laboratory test was 7.57 weeks (1.14-17.29). The mean $\mathrm{Cb}$ was $197 \mathrm{mg} / \mathrm{dL}$ (102-282) and the mean maximum $\mathrm{Ct}$ reached during treatment was $233 \mathrm{mg} / \mathrm{dL}(158$ 333 ), with a difference with $\mathrm{Cb}$ of $36 \mathrm{mg} / \mathrm{dL}$ (DS = 41.84), statistically significant $(\mathrm{p}<0.001)$.

Conclusion: iJAK significantly increased Ct levels, making it necessary in some cases to add or increase statin doses or reduce iJAK doses. We consider that it is a very frequent adverse effect, and it is also a parameter that is little followed, since a high number of patients were excluded from the study due to lack of lipid profile, so it would be necessary to intensify the follow-up of these patients to improve the safety of the treatments.

\section{PDF02.7}

The efficacy and safety of ceftolozane/tazobactam in treatment of bacterial infection: a systematic review and meta-analysis of randomized controlled trials

\section{Qin $\mathrm{Hu}^{*}{ }^{1}$, Bowen Tang ${ }^{2}$, Peng Men ${ }^{3}$}

${ }^{1}$ Pharmacy, Xiangya Hospital, Central South University, Changsha, China, ${ }^{2}$ Xiangya School of Pharmacy, Central South University,
Changsha, Christmas Island, ${ }^{3}$ Department of Pharmacy, Peking University Third Hospital, Beijing, China

Background and Objective: This study aims to assess the clinical efficacy and safety of ceftolozane/tazobactam for the treatment of bacterial infections through meta-analysis.

Method: We conducted a systematic review and meta-analysis of randomized controlled trials (RCTs) to determine the effectiveness and safety of ceftolozane/tazobactam for the treatment of bacterial infections. Electronic databases such as the Cochrane library, EMbase, PubMed, and three Chinese databases were searched for RCTs that compared IBTs with other treatments or placebo. Two reviewers independently assessed the risk of bias, extracted, and analyzed the data. A meta-analysis was performed using Revman 5.3. Publication bias was evaluated.

Main outcome measures: The primary outcomes were the Clinical cure rate and bacterial clearance rate, whereas the secondary outcomes were risk of adverse event and risk of serious adverse events and all-cause mortality.

Results: Seventeen RCTs involving 3938 patients were ultimately included. Ceftolozane/tazobactam had a clinical response rate noninferior to comparators in the treatment of bacterial infection in the microbiologically modified intention-to-treat (mMITT) population, the modified intention to treat (MITT) population and the clinically evaluable (CE) population. The clinical cure rate in the ceftiloza/tazoba group in the microbiologically evaluable (ME) population was better than the control group (meropenem, levofloxacin) [OR=3.88,95\% CI $(2.72,5.53), \mathrm{P}<0.00001]$. ceftiloza/tazobactam had a better bacterial clearance rate in the microbiologically modified intention-to-treat (mMITT) population $[\mathrm{OR}=1.55,95 \% \mathrm{CI}(1.24,1.95)$, $\mathrm{P}=0.0001]$ and microbiologically evaluable (ME) population $[\mathrm{OR}=2.09,95 \% \mathrm{CI}(1.35,3.23), \mathrm{P}=0.0010]$. Furthermore, no significant differences were found between ceftolozane/tazobactam and comparators for the risk of treatment-emergent $\mathrm{AEs}[\mathrm{OR}=1.07,95 \%$ $\mathrm{CI}(0.91,1.26), \mathrm{P}=0.41], \quad$ serious $\quad$ AEs $[\mathrm{OR}=1.25,95 \% \mathrm{CI}(0.87,1.78), \mathrm{P}=0.22] \quad$ or all-cause mortality [OR=1.01,95\%CI $(0.74,1.38), \mathrm{P}=0.97]$.

Conclusion: In the treatment of bacterial infections, ceftaroza/tazobactam has non-inferior or superior efficacy compared with clinical first-line drugs, and is also well.

\section{PDF02.8}

Implementation of medication reviews into a psychiatric hospital: a monocentric, retrospective, observational study

Marie Humbert-Claude ${ }^{*}$, Nader Attalla ${ }^{2}$, Marcos della Paolera ${ }^{2}$, Stephanie Spaggiari ${ }^{1}$, Anne-Laure Blanc ${ }^{1}$, Nicolas Widmer ${ }^{1,3}$

${ }^{1}$ Pharmacie des Hôpitaux de l'Est Lémanique, Rennaz, ${ }^{2}$ La Fondation de Nant, Corsier-sur-Vevey, ${ }^{3}$ Service de Pharmacologie clinique, Centre Hospitalier Universitaire Vaudois et Université de Lausanne, Lausanne, Switzerland

Background and Objective: Psychotropic drugs are complex, associated with potential serious adverse reactions and drug interactions. Comorbidities are frequent among psychiatric population, leading to increased vulnerability and polypharmacy. Optimizing pharmacotherapy is thereby essential. From a drug safety perspective, weekly medication reviews, in a multidisciplinary context (physicians, nurses and a clinical pharmacist) have been implemented within the psychiatric hospital units.

The aim of this study is to describe the pharmaceutical interventions (PI) carried out in a psychiatric hospital and to evaluate the impact on the routine of the clinical pharmacy unit. 
Design: This retrospective, monocentric, observational study, consisted in the analysis of the pharmaceutical interventions (PI) collected from January 2017 to December 2020 during the medication reviews, carried out in 3 adult psychiatric and 1 psychogeriatric units (60 patients). PI have been reported according to the classification system from the Swiss Association of Public Health Administration and Hospital Pharmacists. The number of questions reaching the hotline of the clinical pharmacy unit has also been quantified.

Results: The number of medication reviews increased from 13 in 2017 to 78, 107 and 125 in 2018, 2019 and 2020, respectively. The number of PI were respectively 56, 583, 893 and 870 in 2017, 2018, 2019 and 2020. The main reasons of interventions were medical questions $(23 \%)$, adverse effects $(15 \%)$, inappropriate duration of treatment $(14 \%)$ and interactions $(10 \%)$. The 4 main propositions were the same across 2018, 2019 and 2020 and consisted in providing medication information (20-27\%), dosage adjustments (15-22\%), monitoring (15-18\%) and withdrawal of a medication (14-21\%). Depending on the year, between 59 and $75 \%$ of the propositions were accepted.

Since 2017, the number of hotline questions related to psychiatry was increased by 2-fold in 2018 and 2019 and 3.8-fold in $2020.75 \%$ came from physicians and focused on choice of treatment (19\%), therapeutic alternative (18\%), administration mode (17\%) and dosage $(16 \%)$.

Conclusion: Clinical pharmacists allow to provide safety use of psychotropic drugs and have a central role in improving therapeutic management. The increasing number of questions reaching the clinical pharmacy unit highlights the need of regular pharmaceutical support in psychotropic prescribing.

\section{PDF02.9}

Barriers and enablers to deprescribing in long-term care facilities: a 'best-fit' framework synthesis of the qualitative evidence

\section{Clara Heinrich* $^{\text {1, Maria D. Donovan }}{ }^{1}$, Suzanne McCarthy ${ }^{1}$, Sheena McHugh ${ }^{2}$ ${ }^{1}$ School of Pharmacy, ${ }^{2}$ School of Public Health, University College
Cork, Cork, Ireland}

Background and Objective: Older adults living in long-term care facilities (LTCFs) are amongst the frailest members of society. Their health is challenged through aging and they often experience a substantial medication burden, potentially causing adverse drug reactions. To improve medication safetyand tackle inappropriate polypharmacy, deprescribing is sometimes required, however it is not currently routine practice. This qualitative evidence synthesis aims to summarise the attitudes of healthcare workers (HCWs) toward deprescribing in LTCFs, identifying potential barriers and enablers affecting deprescribing.

Design: The 'best-fit' framework approach was used to synthesise evidence, using the Theoretical Domain Framework (TDF) as the a priori framework. Primary qualitative research investigating HCWs' opinions of deprescribing for older adults in LTCFs was eligible. Included studies were analysed qualitatively and mapped to the TDF, demonstrating LTCF barriers and enablers of deprescribing. Findings which placed greater emphasis on the LTCF context or those which did not fit the TDF were thematically analysed and included. Confidence in findings was assessed using the Grading of Recommendations Assessment - Development and Evaluation and Confidence in Evidence from Reviews of Qualitative Research. A conceptual model was created, hypothesising relationships between domains.
Results: Of 655 records identified, 14 met the inclusion criteria. The 'best-fit' framework identified 17 barriers and 16 enablers which affect HCWs' ability to engage with deprescribing, specific to the LTCF context. The TDF accounted for 22 findings, within eleven domains. Two new domains were developed, patient-centred focus and interprofessional collaboration, supporting 10 findings. The patient-centred focus domain discusses the influence of patients' condition on the deprescribing behaviours of HCWs and the influence of HCWs adopting a patient-centred approach to deprescribing behaviour. Interprofessional collaboration describes the collaborative processes which exist between HCWs, causing changes in attitudes and behaviours to engage with deprescribing. Deprescribing barriers included perceptions of an 'established hierarchy' within LTCFs, negatively affecting communication. Unsupportive social pressures, norms and patient representative input negatively impacts deprescribing. Insufficient resources including staff, finance and documentation limit HCWs' engagement with deprescribing. Enablers include tailored deprescribing guidelines, interprofessional collaboration and working with a patient-centred focus, allowing patients' condition to influence decisions.

Conclusion: This qualitative evidence synthesis has identified that education, interprofessional communication and collaboration can facilitate deprescribing. To overcome the deprescribing barriers, change is required to a patient-centred model, where HCWs are equipped with appropriate resources and adequate reimbursement. The LTCF organisational structure must support deprescribing, with communication and collaboration between healthcare systems.

\section{POSTER DISCUSSION FORM III}

\section{PDF03.1}

Therapeutic drug monitoring of clozapine on patients with treatment resistant schizophrenia

\section{Patrícia Pereirinha* 1, 2, Ana Fortuna ${ }^{2,3}$, José Feio ${ }^{1}$, Eulália Costa $^{4}$, Miguel Bajouco 3 , 5 , Marília Rocha ${ }^{1}$}

${ }^{1}$ Pharmaceutical Services, Coimbra Hospital and University Centre, ${ }^{2}$ Laboratory of Pharmacology, Faculty of Pharmacy, University of Coimbra, ${ }^{3}$ Coimbra Institute for Biomedical Imaging and Translational Research, University of Coimbra, ${ }^{4}$ Department of Clinical Pathology , ${ }^{5}$ Department of Psychiatry, Coimbra Hospital and University Centre, Coimbra, Portugal

Background and Objective: Clozapine (CLZ) is the most effective drug for treatment resistant schizophrenia, but haematological lifethreatening effects and the rigorous analytical control ends up in high discontinuation rates as well a delayed treatment onset ${ }^{1}$. Therefore, measuring plasma CLZ concentrations is useful to optimize therapy and minimize toxicity. Herein, intra- and inter-individual characteristics influence on CLZ concentrations were investigated.

Method: This retrospective study consisted on analyzing plasma concentrations of CLZ and norclozapine (NCLZ) collected from patients followed on a Treatment Resistant Schizophrenia Advanced Care Unit from Coimbra Hospital and University Centre, between January 2017 and October 2019. Gender, age, weight, smoking behavior, comedicated drugs, daily doses (D) and metabolic ratios were collected and analyzed through IBM ${ }^{\circledR}$ SPSS Statistics 27.

Main outcome measures: Categorization of CLZ plasma concentrations into sub-therapeutic, therapeutic and supra-therapeutic; the correlation coefficients between CLZ concentrations and patient variables.

Results: The tested population $(n=60)$ presented an irregular distribution ( 3 women and 57 men), with $62 \%$ of smokers, $15 \%$ of nonsmokers and $23 \%$ without information about smoking habits. With a 
total of 269 plasma CLZ concentrations, the CLZ mean \pm standard deviation D was $372 \pm 173 \mathrm{mg}$, while CLZ and NCLZ concentrations were $336 \pm 229 \mathrm{ng} / \mathrm{mL}$ and $176 \pm 110 \mathrm{ng} / \mathrm{mL}$, respectively. Approximately, $61 \%$ of the concentrations were sub-therapeutic $(<350 \mathrm{ng} /$ $\mathrm{mL}), 30 \%$ were within the therapeutic range $(350-600 \mathrm{ng} / \mathrm{mL})$ and $9 \%$ above $(>600 \mathrm{ng} / \mathrm{ml})$. The mean metabolic ratio was $1.99(>1.32)$, suggesting a non-through value, poor metabolizers or the presence of metabolic inhibitors ${ }^{2}$. In 232 CLZ plasma concentrations ( $86 \%$ ), the CLZ was administered together only with drugs that inhibit isoforms of cytochrome P450. CLZ/D values were smaller in smokers (0.78) than in non-smokers (1.79), proving that smokers require higher doses to achieve the therapeutic level due to their enhanced clearance, as described for the total CLZ/D ratio $(1.20 ; 2.55)$. CLZ concentrations showed a moderate correlation with smoking status $(\mathrm{r}=-0.33)$ and weight $(\mathrm{r}=0.34)$, and better a correlation with total $\mathrm{C} / \mathrm{D}$ ratio $(\mathrm{r}=-0.51$; $\mathrm{r}=0.47$ ). On the other hand, comedication, gender and age did not show a significant correlation with CLZ concentrations.

Conclusion: Weight and smoking habits must be considered in clozapine therapeutic monitoring in order to achieve the expected therapeutic response.

References: 1.Farooq S, Choudry A, Cohen D, Naeem F, Ayub M. Barriers to using clozapine in treatment-resistant schizophrenia: systematic review. BJPsych Bull. 2019;43(1):8-16. doi:10.1192/ bjb. 2018.67

2.M.MeyerJonathan MSS. The Clozapine Handbook-Stahl's Handbooks. Cambridge, United Kingdom: CAMBRIDGE UNIVERSITY PRESS; 2020. doi:10.1017/9781108553575

\section{PDF03.2}

Long-acting injectable second-generation antipsychotics: conditions of reimbursement after repeated hospitalization and estimated the budgetary impact

\section{Ivana Minarčíková* 1 \\ ${ }^{1}$ HTA and Market Access, HTA Pharmacy s.r.o., www.HTApharmacy.cz, Brno, Czech Republic}

Background and Objective: State regulatory agency in the Czech Republic, The State Institute for Drug Control (SÚKL) sets maximum prices, reimbursements and conditions of reimbursement for pharmaceuticals. Reimbursement conditions are restrictions on the prescription of the pharmaceutical. Long-Acting Injectable SecondGeneration Antipsychotics (LAI-2) can be prescribed, if the patient meets several conditions, one of which is repeated hospitalization for relapse due to non - adherence (nonA) to treatment. Only oral antipsychotics are reimbursed until the repeated hospitalization occurs. However, their use is associated with a high degree of nonadherence, and is the reason for $50-55 \%$ of hospitalizations. ${ }^{1,2}$

The aim of the work was to estimate whether the setting conditions of reimbursement for LAI-2 are cost savings for the Czech health budget.

Method: The direct annual costs of health insurance companies (ZP) per every LAI- $2^{3}$ were processed using the cost-minimization analysis (CMA) and the amount of costs for hospitalization for schizophrenia was found. $^{4,5}$ Subsequently, Budget Impact Analysis (BIA) compared the cost of LAI - 2 and hospitalization in relation to different levels of nonA. Work was worked conservatively, not including drug administration costs, switch costs for other therapy/ pharmaceuticals and treatment of adverse events.

Main outcome measures: Work compared the annual cost of LAI 2 , the variance of nonA and the consequent total cost of hospitalization.
Results: The CMA calculated that the cost ranged from $152.55 \mathrm{CZK}$ to $254.34 \mathrm{CZK}$ per defined daily dose LAI-2. The maximum annual cost of LAI - 2 for one patient reached an average value of 75.976 CZK, ranging from $55.682 \mathrm{CZK}$ to $92.835 \mathrm{CZK}$.

It was completed 3.475 hospitalizations (dg. F2) with an average duration of hospitalization of 19.8 days and a total cost for one patient 89.327 CZK (median) per DRG Group Acute psychiatric care for 16-20 days for mental illness. ${ }^{4,5}$ But real cost may vary according to the contractual arrangements of each individual hospital with the ZP.

BIA calculated a different degree of nonA and calculated what the budgetary impact would be if a non-adherent patient used LAI-2 and did not incur hospital costs. The BIA results showed that savings could be achieved from CZK -25.5 million (at 55\% nonA) to CZK -2.3 million (at 5\% nonA).

In the sensitivity analysis the BIA estimated a cost saving impact (CZK -64.3 million to CZK-5.8 million) at a minimum annual cost of LAI-2 (CZK 55,682.05) and an acceptable budget impact of $6.7 \mathrm{CZK}$ million to CZK 0.6 million) at the maximum annual cost of LAI-2 (CZK 92,835.38).

Conclusion: The estimated budgetary impact showed that the current cost of LAI-2 pharmaceuticals is low enough that the setting of reimbursement conditions isn't cost savings for the Czech health insurance. The estimated BIA has its limitations (conservative approach to the CMA, to the costs of hospitalization and follow-up care, the harm and lower quality of life in repeated hospitalization), but points to an unnecessary restriction on the reimbursement of LAI2.

References: 1 . Perkins DA et al. Predictors of antipsychotic medication adherence in patients recovering from a first psychotic episode. Schizophr Res. 2006 Mar;83(1):53-63. doi: 10.1016/ j.schres.2005.10.016. Epub 2006 Mar 9.

2. Kane JM et al. Optimizing Treatment Choices to Improve Adherence and Outcomes in Schizophrenia. J Clin Psychiatry 2019;80(5):IN18031AH1C

3. Seznam cen a úhrad LP/PZLÚ k 1. 6. 2021. Státní ústav pro kontrolu léčiv. [on line] Dostupné z: https://www.sukl.cz/

4. Melicharová H. et al. Psychiatrická péče 2019. Zdravotnická statistika. Ústav zdravotnických informací a statistiky ČR. 2021. ISSN 1210-8588. ISBN 978-80-7472-188-5. [on line] Dostupné z: https://www.uzis.cz

5. Klasifikační systém CZ-DRG 3.0 revize 1. [on line] Dostupné z: https://www.drg.uzis.cz

\section{PDF03.3}

Content validation of an algorithm for the assessment, management and monitoring of drug-induced QTC prolongation in the psychiatric population

\section{Shorouq Homes ${ }^{*}{ }^{1}$, Monica Zolezzi ${ }^{1}$, Waad Elamin ${ }^{1}$, Iman Qubaiah $^{1}$, Doaa Mahmoud ${ }^{1}$, Athar Elhakim ${ }^{2}$ \\ ${ }^{1}$ College of Pharmacy, QU Health, Qatar University, ${ }^{2}$ Pharmacy, College of North Atlantic, Doha, Qatar}

Background and Objective: QTc interval prolongation leads to serious complications, making it a concern for all clinicians. Assessing the risk of QTc prolongation in the psychiatric population is particularly important because they are exposed to multiple medications known to increase the risk of life-threatening arrhythmias. Care paths in the form of algorithms have been developed to guide clinicians at the time of prescribing to minimize this risk. As such, the objective of this study was to assess the content validity of an 
algorithm for the assessment, management and monitoring of druginduced QTc prolongation in the psychiatric population.

Design: A cross-sectional, anonymous, self-administered survey was embedded in an online orientation module to the algorithm (referred to as QTc Prolongation Algorithm or QTcPA) and was given to mental health practitioners (MHPs) such as pharmacists and physicians. The online survey included quantitative and qualitative components to gather feedback on the relevance and appropriateness of each step in the algorithm. The quantitative component included the estimation of the content validity index (CVI) for each step of the algorithm. Word-clouding was used in the qualitative analysis of the MHPs responses to the open-ended questions on the survey.

Results: Results showed an average mean CVI scores ranging from 3.68 to 3.86 out of 4 for the appropriateness of the QTcPA's steps, 3.68 to 3.81 for the safety and 3.5 to 3.77 for the reliability of references used in the algorithm. Individual item-CVI analysis indicated high validity of the QTcPA as it scored 0.86 to 1 for all of the steps/ decision statements in the three aspects assessed: appropriateness, safety, and reliability of references used. The open-ended questions results indicated MHPs supported the implementation of the QTcPA in practice; however, they recommended simplification of the steps and provision of the online orientation module for training on its use. Conclusion: Results of this study validate the steps in the QTcPA and suggest that its implementation in practice may prove to be a useful tool in assisting MHPs in the decision-making process at the time of prescribing medications with risk of QTc prolongation.

\section{PDF03.4}

Influenza vaccination coverage of Belgian patients using tumor necrosis factor-alpha-inhibitors between 2015 and 2019

Silas Rydant* 1, 2, 3, Lara Czuba ${ }^{4}$, Hans De Loof ${ }^{1}$, Peggy Jacques ${ }^{5}$, Bart Van den Bemt ${ }^{6}$, Stephane Steurbaut ${ }^{2}$

${ }^{1}$ Laboratory of Physiopharmacology, Department of Pharmaceutical Sciences, University of Antwerp, Antwerpen, ${ }^{2}$ Research group of Clinical Pharmacology and Clinical Pharmacy, Vrije Universiteit Brussel/UZ Brussel, Brussels, ${ }^{3}$ Koninklijke Apothekersvereniging Antwerpen (KAVA), Antwerpen, ${ }^{4}$ Vrije Universiteit Brussel/UZ Brussel, Brussels, ${ }^{5}$ Department of Rheumatology, Ghent University Hospital, Ghent, Belgium, ${ }^{6}$ Department of Pharmacy, Radboud University Medical Centre, Nijmegen, Netherlands

Background and Objective: Tumor necrosis factor-alpha-inhibitors (TNF-inhibitors) are used to control disease activity in different autoimmune diseases such as rheumatoid arthritis, Crohn's disease, psoriasis or uveitis. These drugs however make patients more prone to infections, including influenza. Different national and international guidelines therefore recommend influenza vaccination but currently the vaccination coverage in Belgium is unknown among these patients. This study investigates the influenza vaccination coverage in Belgian patients using TNF-inhibitors.

Method: A retrospective study was carried out using BelpharData, a database containing dispensing data of reimbursed drugs originating from $\sim 80 \%$ of the Belgian community pharmacies. Data regarding influenza vaccine prescriptions among patients on TNF-inhibitors was collected over a period of 5 years (1 January 2015-31 December 2019). Data about the patients' age, type of prescriber and type of TNF-inhibitor, were also collected.

Main outcome measures: The percentage of Belgian patients using TNF-inhibitors that is vaccinated against influenza between 2015 and 2019.

Results: The influenza vaccination coverage in Belgian patients on TNF-inhibitor therapy in ambulatory care fluctuated between $36.9 \%$
(2015) and $39.1 \%$ (2018). The aggregated influenza vaccination coverage from 2015 to 2019 was $42.9 \%$ in Flanders, 32.9\% in Wallonia and $23.6 \%$ in the Brussels Capital Region. Patients using TNFinhibitors for rheumatic diseases had the highest influenza vaccination coverage $(41.2 \%)$ compared to gastroenterological $(34.0 \%)$, ophthalmological $(32.0 \%)$ and dermatological (26.3\%) diseases. Etanercept was the drug with the highest influenza vaccination coverage from 2015 to 2019 (44.1\%), followed by certolizumab (40.8\%), golimumab $(36.4 \%)$ and adalimumab $(34.8 \%)$. Most influenza vaccines were prescribed by the general practitioner (83.6\%). The average influenza vaccination coverage in the age group $\geq 71$ years was $70.7 \%$, whereas this was below $40 \%$ for patients between 11 and 60 years.

Conclusion: The influenza vaccination coverage in Belgian patients using a TNF-inhibitor is inadequate given the increased risk of infections. Influenza vaccination rates differed markedly by geographical region, age and type of drug. Patients with rheumatic diseases had a higher influenza vaccination coverage compared to other disorders for which TNF-inhibitors are used. Limitation to our study are that in-company-vaccinations were not registered and that the results were based on dispensing data and not on vaccine administration data. Further implementation of guidelines and influenza vaccination campaigns are necessary to improve influenza vaccination coverage especially in patients younger than 61 years.

\section{PDF03.5}

The incidence of metabolic syndrome amongst Qatar migrants 24 months post-migration: a prospective longitudinal observational cohort study

Rana Al-Adawi* 1, 2 , Kirti. S. Prabhu ${ }^{3}$, Derek Stewart ${ }^{4}$, Cristin Ryan $^{5}$, Hani Abdelaziz ${ }^{6}$, , Mohsen EL Edrisi ${ }^{7}$, Mohamed Izham Mohmed Ibrahim ${ }^{8}$, Shahab Uddin ${ }^{7}$, Antonella Tonna ${ }^{1}$

${ }^{1}$ School of Pharmacy and Life Sciences, Robert Gordon University, Aberdeen, United Kingdom, ${ }^{2}$ Pharmacy department , ${ }^{3}$ Translational Research Institute, Academic Health System, Hamad Medical Corporation, ${ }^{4}$ College of Pharmacy, QU Health, Qatar University , Doha, Qatar, ${ }^{5}$ School of Pharmacy and Pharmaceutical Sciences, Trinity College, Duplin, United Kingdom, ${ }^{6}$ Vitalité Health Network, New Brunswick, Canada, ${ }^{7}$ Internal Medicine department, Hamad Medical Corporation, ${ }^{8}$ Pharmacy department, Qatar University, Doha, Qatar

Background and Objective: Evidence indicates that migration to Western countries is associated with increased metabolic syndrome (MetS) risk. There is, however, a scarcity of data about MetS incidence in migrants to Middle Eastern countries. This study aimed to investigate the relationship between migration and the incidence of MetS following a 24-months residency in Qatar.

Method: Following the necessary ethics approvals, migrants to Qatar aged 18-65 years were invited to participate. Baseline screening for MetS parameters included glycated haemoglobin, triglycerides, highdensity lipoprotein-cholesterol, blood pressure, and waist circumference. Migrants with normal metabolic parameters were invited for rescreening 24-months post-migration and, parameters repeated. Those with abnormal metabolic parameters were counselled or referred for medical review and excluded from follow up.

Main outcome measures: The incidence of metabolic syndrome amongst initially metabolic syndrome-free moigratns, 24-months post migration.

The determinants of MetS andMetS elements among Qatar migrants, 24 months post migration. 
Results: Four hundred seventy-two consented to participate of 1379 identified. 205 (43.4\%) migrants had normal metabolic parameters at baseline and were recalled 24 months post-migration, with 160 completing follow-up. The incidence of MetS within this group rose to $17 \%(\mathrm{n}=27 / 160,95 \% \mathrm{CI} ; 11.0 \%-23.0 \%)$ and $81 \%(\mathrm{n}=129 / 160)$ developed at least one element of MetS following 24 months in Qatar.

Conclusion: Migration to Qatar was associated with the development of MetS after 24 months of migration. Further studies are required to determine the risk factors and the predictors of MetS amongst migrants to Qatar.

\section{PDF03.6}

Evaluation of the added value of arginine AP-HP $6.25 \%(\mathrm{w} / \mathrm{v})$ hospital preparation in the exploration of somatotropic function: observational use survey

Raul Diaz Salmeron ${ }^{*}{ }^{1}$, Cécilia N'Guessan ${ }^{1}$, Jean-Jacques Houri ${ }^{1}$, Valentin Pouilleau ${ }^{1}$, Marie-Pierre Berleur ${ }^{1}$ on behalf of Département Affaires Réglementaires, Pharmaceutiques et Médicales, Etablissement Pharmaceutique de l'Assistance Publique-Hôpitaux de Paris, AGEPS

\section{${ }^{1}$ AGEPS, AP-HP, Paris, France}

Background and Objective: The somatotropic axis plays an essential role in growth and metabolism due to hormonal mediators, including growth hormone (GH). Indeed, GH is actively involved in various metabolic, reproductive and growth phenomena and can be the root cause of metabolic and hormonal disorders. Its evaluation, as well as that of the right somatotropic axis function, remains essential for the diagnosis of several diseases such as dwarfism, gigantism or acromegaly.

In this context, our pharmaceutical establishment provides the Hospital Preparation (HP) ARGININE AP-HP $6.25 \%$ (w/v), solution for intravenous infusion. It is used for performing dynamic tests to explore somatotropic function, more specifically $\mathrm{GH}$ stimulation tests.

The main objective was to assess the added value of this HP to confirm or deny its essential character in the aforementioned indication. Thus, an observational survey on the use in endocrinology regarding the exploration of somatotropic function was carried out in hospitals that purchased this preparation in 2020 .

Design: The survey was mailed on September 21, 2021 to the 85 hospitals that purchased this HP in 2020. It was focused on the use of this HP for a "dynamic test to explore somatotropic function". A follow-up was carried out on October 1, 2020. After the investigation was closed on October 6, 2020, an acknowledge e-mail was sent to the 85 hospitals initially consulted.

Results: The survey's response rate was $15.3 \%$ (13 responses out of 85 hospitals contacted). The results remain significant because we note among the various responders several hospitals performing more than 100 somatotropic axis tests per year. In addition, the responders who sent back the survey constitute a large part of the annual sales of this HP in 2019 (28.6\%). Two thirds of the hospitals that responded to the survey use Arginine AP-HP (alone and/or in combination) for the claimed indication in this work. All except one (85.7\%) highlight its essential character for diagnostic purposes. Indeed, arginine is described in the scientific literature as a potent GH secretagogue due to the inhibition of somatostatin secretion and the increase of the endogenous GH biosynthesis leading to an increase in the GH-mRNA expression. In half of the cases, this HP is used in combination with insulin to improve the test sensitivity.
Conclusion: Most of the hospitals responding to our survey highlighted the added value of this HP in the claimed therapeutic indication. This is mainly related to the low number of contraindications, the safety profile and the test sensitivity. Our results are aligned with scientific data in the literature. In order to demonstrate the somatotropic axis dysfunction, pharmacological stimulation tests are currently the golden rule. The arginine test, alone or in combination, is one of the most commonly used tests.

\section{PDF03.7}

Lithium's prescription in acute mania: retrospective study of psychiatry's prescriptions in a French hospital

Fany Roger ${ }^{*}$, Jean-Marie Desce ${ }^{2}$

${ }^{1}$ Université de Paris, Paris, ${ }^{2}$ GHEF, Jossigny, France

Background and Objective: Lithium is the empiric treatment of acute mania. This mood stabilizer is known for its anti-maniac properties. But now, some other molecules such as antiepileptics and APA have the same properties.

International guidelines from several learned societies recommend monotherapy in first line to treat acute mania and especially lithium, APA or valproate. In clinical practice, are these recommendations followed and what is the place of lithium?

Method: In this study we investigated all the treatments prescribed in acute mania between July $1^{\text {st }} 2019$ and June $30^{\text {th }} 2020$ in 4 psychiatric units of a French hospital. During this period, 1015 patients were hospitalized, among them, 149 for acute mania (ICD-10: F30.0, F30.1, F30.2, F30.8, F30.9, F31.0, F31.1, F31.2). Pediatric and geriatric populations were excluded from the study. Datas were extracted from MEVA-Crossway and we collected it in Excel. Analysis was made with the same program.

Main outcome measures: Among the 149 inpatients of the study, only $29.5 \%$ were treated with lithium. While $75 \%$ of them received a combination of lithium plus an other mood stabilizer, only $1.34 \%$ of all the studied inpatients received a strict lithium monotherapy.

Results: 33 different psychotropic drugs except hypnotics were used. Polypharmacy was predominant and frequently observed for the treatment of acute mania with a mean of $2.75+/-1.15$ psychotropic drugs. Almost half the patients received a dual therapy and $10 \%$ a triple therapy. Only $35 \%$ of the inpatients received a mood stabilizer monotherapy.

Surprisingly, 9 out 149 inpatients had an antidepressant whereas it is not recommended in the international guidelines.

Even if sleeping troubles are not considered in international guidelines, hypnotics for sleeping troubles were frequently prescribed $(52.3 \%)$.

To manage agitation, psychiatrists used in addition to mood stabilizers tranquilizers such as loxapine, haloperidol and benzodiazepines.

Atypical antipsychotics seem to become the most used treatment in clinical practice compared to the historic treatments (lithium and antiepileptics) with 110 prescriptions (it does not mean that 110 inpatients had an APA because some of them had a dual or more therapy). Although $13.4 \%$ of the inpatients received APA IM whereas they do not have the authorization for this indication in France.

Conclusion: Lithium seems to be abandoned in favor of APA or antiepileptics. Its narrow therapeutic window, its necessary regular monitoring and its side effects may be the principal reasons of this evolution. 


\section{PDF03.8}

Healthcare providers' perspective and experiences with insomnia management at primary healthcare centers in Qatar

\author{
Raja Ali ${ }^{*}{ }^{1}$, Monica Zolezzi ${ }^{1}$, Ahmed Awaisu ${ }^{1}$ \\ ${ }^{1}$ College of Pharmacy, QU Health, Qatar University, Doha, Qatar
}

Background and Objective: Insomnia is a public health concern that affects one in every three adults and is the second most common complaint at primary healthcare settings. In Qatar, Primary Health Care Centers (PHCCs) are often patients' the first point of contact with the healthcare system. This study aimed to explore health care providers' (HCPs) perspective of insomnia and their role in its management.

Design: This was a qualitative study which used semi-structured interviews with pharmacists and physicians working at PHCCs for a minimum of one year to explore their views on insomnia and its management. Interviews were transcribed and thematically analysed. Results: Saturation was achieved after 19 HCPs were interviewed. The interviews generated five themes, including general perspectives on insomnia, primary healthcare as the setting for insomnia management, current practices for insomnia management at PHCCs, HCPs' role perception, and challenges facing insomnia management. HCPs viewed insomnia as a symptom of an underlying health problem, largely manageable at PHCCs. Participants reported limited availability of treatments at PHCCs for managing insomnia, leading to the use of other less specific classes of medications (e.g., antihistamines). HCPs generally preferred non-pharmacological over pharmacological treatments for managing insomnia. Physicians viewed the assessment and management of insomnia as their role, while pharmacist viewed their role was the provision of medicationrelated information and counselling. Time constraints and lack of insomnia guidelines were the main challenges highlighted by HCPs. Conclusion: HCPs viewed insomnia as an important health concern among people attending PHCCs, but indicated that the care provided was limited by the restricted availability of insomnia treatments and sparse management guidelines.

\section{PDF03.9}

Insomnia, cognitive impairment and inappropriate patterns of hypnosedative drug use in older patients in acute care: outputs from the inomed and the EUROAGEISM projects

Olena Antonenko, MD* 1 , Andrea Zelinková, MSc ${ }^{1}$, Milada Halačová, PharmD ${ }^{1}$, Silvia Grešáková, MSc ${ }^{1}$, Jovana Brkic, MSc $^{1}$, Daniela Fialová, PharmD, Ph.D, Assoc. Prof. ${ }^{1}$

${ }^{1}$ Department of Social and Clinical Pharmacy, Faculty of Pharmacy in Hradec Králové, Charles University, Prague, Czech Republic

Background and Objective: Previous meta-analysis demonstrated that insomnia(particularly high sleep fragmentation)is associated with a 1.5 -fold risk of developing dementia and a $22 \%$ increase in the annual rate of cognitive decline. Sleep disturbances and cognitive impairment are inter-connected and insomnias in older patients are very frequent particularly in acute care due to multiple aggravating risk factors. Interventions to decrease insomnias and inapproriate use of hypnosedatives may therefore help in reducing the risk of dementia and cognitive decline. The aim of our study was to determine the prevalence of insomnias in acutely hospitalized older patients, particularly in adults suffering from cognitive impairment, dementia or acute deliria and to investigate patterns of inappropriate hypnosedative drugs use in acute geriatric care in the Czech Republic.
Method: 438 older patients ( $\geq 65 \mathrm{yrs}$ ) acutely hospitalized at 3 geriatric clinics in the Czech Republic underwent a comprehensive geriatric assessment(CGA) using the EUROAGEISM H2020 assessment protocols. Explicit criteria of potentially inappropriate medications (PIMs), mainly 2019 Beers criteria and 2015 EU(7)-PIM list were applied to determine inappropriate patterns of hypnosedative drugs use.

Main outcome measures: Insomnia has been diagnosed in $16.9 \%$ $(\mathrm{N}=74)$ patients in the total sample and in $40,5 \%(\mathrm{~N}=30)$ of seniors diagnosed dementia. Very mild cognitive decline was assessed in $21,6 \%(\mathrm{~N}=16)$, mild or moderate cognitive - in $14,9 \%(\mathrm{~N}=11)$ and $1,3 \%(\mathrm{~N}=1)$ older patients, respectively; delirium in $23 \%(\mathrm{~N}=17)$ of subjects. Use of hypnosedatives in the evening or at night $(\mathrm{e} / \mathrm{n})$ was much more prevalent $(34,6 \%)$ than the recorded diagnosis of insomnia in $16,9 \%$. Moreover, $13.8 \%(\mathrm{~N}=10)$ of older adults reported e/n use of drugs aggravating insomnias (particularly beta-blockers $6.4 \%$, diuretics $2.5 \%$, theophylline $2.1 \%$ )

Results: Most frequent hypnosedatives drugs used were: antipsychotics e/n(18.5\%), Z-drugs $(16,2 \%$, in $10,5 \%$ used in higher doses) and benzodiazepines e/n(BZD $14,2 \%$, in $5,3 \%$ used for long term).The monotherapy by hypnosedatives was recorded in $42 \%$ $(\mathrm{N}=184)$ of cases, combination therapy (2 hypnosedatives) in $5,7 \%$ $(\mathrm{N}=25)$ of seniors. Non-geriatric doses were prescribed particualrly in users of Z-drugs $(10.5 \%)$ and sedative antidepressants e/n (1,8\%), longer than recommended duration of geriatric therapy has been documented mainly in Z-drugs $(5.9 \%,>1$ month $)$, BZDs e/n $(5,3 \%,>1$ month), sedative antidepressants $\mathrm{e} / \mathrm{n}(3,3 \%>6$ months).

Conclusion: In our study, often demented and delirious patients had recorded in their medical records a diagnosis of insomnia and/or the use of hypnosedatives. Inappropriate patterns of hypnosedative drugs use in acute care were confirmed particularly in frequent indications of antipsychotics e/n, inappropriate dosing of Z-drugs and long-term use of BZDs e/n. Grants:EuroAgeism Horizon 2020 MSCF-ITN764632, Inomed NO.CZ.02.1.01/0.0/0.0/18_069/0010046, Progress Q42- Faculty of Pharmacy, Charles University, START/MED/093 CZ.02.2.69/0.0/0.0/19_073/0016935, SVV260 551 and I-CARE4OLD H2020 -965341

References: Lim AS, Kowgier M, Yu L, Buchman AS, Bennett DA: Sleep fragmentation and the risk of incident Alzheimer's disease and cognitive decline in older persons. Sleep. 2013, 36:1027- 1032. 10.5665/sleep. 2802

\section{POSTERS}

\section{PP001}

Prescription of sedatives and sedative burden of drug regimens in seniors in Spain and the Czech Republic: results from the INOMED and the EUROAGEISM $\mathrm{H} 2020$ projects

Adriana Magátová* ${ }^{1}$, Adriana Slaná ${ }^{1}$, Jindra Reissigová $^{1,2}$, Anna Lukačišinová $^{1}$, Jovana Brkic ${ }^{1}$, Daniela Fialová ${ }^{1,3}$

${ }^{1}$ Department of Social and Clinical Pharmacy, Faculty of Pharmacy in Hradec Králové, Charles University, Hradec Králové, ${ }^{2}$ Department of Statistical Modelling, Academy of Science, ${ }^{3}$ Department of Geriatrics and Gerontology, 1st Facuty of Medicine, Charles University, Prague, Czech Republic

Background and Objective: The sedative potential of drugs is accented in seniors by pharmacodynamic and/or pharmacokinetic changes and sedative burden of drug regimens should be minimized in older adults. Higher response to sedative drugs may be stimulated also by polypharmacotherapy, alcohol intake and/or some comorbidities. The aim of our pilot study was to identify and compare the 
prescription of sedative drugs in older adults in Spanish (SP) and Czech (CZ) samples participating in the EuroAgeism H2020 project. Method: Data of 260 SP community-residing seniors 65+ (from community pharmacy practices - CPP) and of $1602 \mathrm{CZ}$ seniors 65+ from 3 settings of care (acute $=589$, ambulatory $=563$ and $\mathrm{CPP}=450$ ) were prospectively collected in the EuroAgeism $\mathrm{H} 2020$ project (2018-2019) in regionally different study sites using Comprehensive Geriatric Assessment protocol. Pilot results were analyzed with descriptive statistics (R-software, vers. 4.0.3).

Main outcome measures: Prevalence of use of medications with various sedative potential in $\mathrm{SP}$ and $\mathrm{CZ}$, comparison of sedative potential of prescribed drug combinations (based on literature research of sedative drug potential).

Results: In SP and CZ samples (CPP), there were $62.4 \%$ and $64.6 \%$ of women and the mean age of participants was $71.74 \pm 6.25 \mathrm{SD}$ and $76.61 \pm 7.15 \mathrm{SD}$, respectively. Polypharmacy/excessive polypharmacy were documented in 54.7\%/24.9\% and 47.4\% / 3.8\% (p<0.001) in CZ sampleand in $41.2 \% / 13.1 \%(\mathrm{p}<0.001)$ in CPP in SP. In the CZ sample,the highest prevalence of sedative drug was confirmed in acute care $(56.5 \%)$, compared to ambulatory care (49.0\%). In CPP, $47.3 \%$ of seniors used at least 1 sedative drug in SP compared to $11.1 \%$ in $\mathrm{CZ}(\mathrm{p}<0.001)$. At least 2 sedative drugs combined were prescribed in $24.4 \%, 22.2 \%, 1.8 \%$ of seniors in acute, ambulatory care and CPP in CZ, and in $15 \%$ in CPP in SP. The combination of $3+$ sedatives was documented in $7,8 \%, 8,2 \%$ and $0.7 \%$ in CZ samples, respectively and in $4.2 \%$ in SP CPP. The most frequent combinations of sedatives were: in SP-lorazepam and citalopram (9.4\%, very strong sedative potential-4.5) and diazepam and citalopram (9.4\%, similar sedative potential-4); in CZ alprazolam and citalopram $(5.0 \%$, sedative potential- 3.0) and citalopram and bromazepam (3.3\%, sedative potential-4.5).

Conclusion: Our descriptive findings confirmed significant differences in sedatives drugs prescribed in monotherapy or combinations to seniors in SP and CZ in CPP (with higher burden in CPP in SP) as well as significant differences accros different settings of care (with the highest prevalence in acute care). Careful drug selection might help to reduce sedative drug regimens burden in older population.

Grants: EuroAgeism Horizon 2020 MSCF-ITN-764632, Inomed NO.CZ.02.1.01/0.0/0.0/18_069/0010046, Progress Q42- Faculty of Pharmacy, Charles University, START/MED/093 CZ.02.2.69/0.0/0.0/ 19_073/0016935, SVV260 551 and I-CARE4OLD H2020 -965341

\section{PP002}

Brief interventions targeting long-term benzodiazepine receptor agonist use in primary care: a simple solution for a complex problem?

\section{Aisling Barry ${ }^{*}$, Simon Lewin ${ }^{2,}$, Cathal Cadogan ${ }^{1}$ \\ ${ }^{1}$ School of Pharmacy and Pharmaceutical Sciences, Trinity College Dublin, Dublin, Ireland, ${ }^{2}$ Division of Health Services, Norwegian Institute of Public Health, Oslo, Norway, ${ }^{3}$ Health Systems Research Unit, South African Medical Research Council, Cape Town, South Africa}

Background and Objective: Benzodiazepine receptor agonists (BZRAs) are commonly prescribed on a long-term basis, despite clinical guidelines advocating that they should be limited to shortterm use ( $<4$ weeks) due to risks of dependence and adverse events. A recent systematic review (Lynch et al. Addiction. 2020;115(9):161839) found that brief interventions targeting long term BZRA use in primary care (e.g. short consultations with healthcare professionals, letters to patients) were effective in helping patients to change their behaviour and discontinue the medication. However, the complexity of these interventions and implications on effect size has not been comprehensively assessed. This study aimed to apply the intervention Complexity Assessment Tool for Systematic Reviews (iCAT_SRLewin et al. BMC Med Res Methodol. 2017;17(1):76) to brief interventions targeting long-term BZRA use.

Design: Interventions from the existing systematic review were assessed independently by two reviewers using the six core iCAT_SR dimensions: organisational level/category targeted, behaviour targeted, number of intervention components, degree of tailoring, level of skill required by those delivering the intervention and level of skill required by those receiving the intervention. The four optional iCAT_SR dimensions were applied to the interventions where possible. A scoring system was using to calculate a complexity score for each intervention. Pearson's correlations were used to assess the relationship between intervention complexity and effect size, as well as the relationship between the number of identified behaviour change techniques (BCTs) and intervention complexity. Inter-rater reliability was calculated using Cohen's Kappa coefficient.

Results: The six core iCAT_SR dimensions were applied to the interventions with high inter-rater reliability (Cohen's Kappa=0.916). Application of the four optional dimensions was prevented by a lack of detail in study reports. Intervention complexity scores ranged from 9 to 12 (median=12). There was no relationship detected between intervention complexity and effectiveness. There was also no relationship found between number of identified BCTs and intervention complexity.

Conclusion: This is the first study to examine the complexity of brief interventions targeting long-term BZRA use. The findings indicate that complexity arises in these interventions due to the use of multiple components, the degree of flexibility allowed in how the interventions are conducted and the skills which are required by those delivering and receiving these interventions in order for the intervention objective to be achieved.

\section{PP003}

What are the key elements to assess the quality of medication review type 3?

Anneleen Robberechts ${ }^{*}$ 1, 2, Melissa Michielsen ${ }^{3}$, Silas Rydant ${ }^{1,}$, ${ }^{4}$, Stephane Steurbaut ${ }^{4}$, Guido De Meyer ${ }^{2}$, Hans De Loof ${ }^{5}$

${ }^{1}$ Koninklijke Apothekersvereniging Antwerpen (KAVA), ${ }^{2}$ Laboratory of Physiopharmacology, Department of Pharmaceutical Sciences, University of Antwerp, ${ }^{3} /$, Antwerpen, ${ }^{4}$ Research group of Clinical Pharmacology and Clinical Pharmacy, Vrije Universiteit Brussel/UZ Brussel, Brussels, ${ }^{5}$ Laboratory of Physiopharmacology, University of Antwerp, Antwerpen, Belgium

Background and Objective: Medication review is a structured evaluation of a patient's medicines with the aim of optimising medicines use and improving health outcomes. This entails detecting drug related problems and recommending interventions. Carrying out MR3 requires high quality to implement this service in Belgian community pharmacies. Currently, there is no instrument or tool to assess the overall quality. This study aims to develop a tool that can assess the quality of MR3.

Method: A list of 57 criteria was developed in Dutch and divided in 9 subdomains (eg. focus on patient or therapy). This list was sent electronically to the Dutch PRISMA-network, Flemish pharmacy students, Flemish pharmaceutical care researchers and Flemish pharmacists with prior experience in performing MR3. Participants needed to rank the criteria in order of perceived importance. The drag-and-drop ranking method was used, with no ex aequo allowed. 
Results were analyzed using SPSS, Mann Whitney U and the Kruskall-Wallis-test.

Main outcome measures: A list of important criteria that can serve as a tool to assess the quality of MR3.

Results: In total 106 respondents (42 Dutch and 64 Flemish respondents) completed anonymously the survey. The top 5 of most important criterias was discussing the usefullness and goals of MR3 with the patient, evaluating and discussing current indications and drugs, assessing if patients can swallow their medication and providing an up to date medication overview. Criteria that were perceived less important are the cost and expiry date of drugs, the risk of addiction, how patients feel about injections and discussing nonpharmacological therapy. Participants with more experience with MR3 reported the use of reliable tools as more important compared to less experienced participants. Participants with more experience in community pharmacy perceived reporting to the GP as more important and the treatment plan as less important compared to participants with less or no experience in community pharmacy. Flemish pharmacists ranked drug-drug interactions higher compared to Dutch pharmacists and dosing compatibility with renal impairment as less important compared to Dutch pharmacists.

Conclusion: Although respondents found it difficult to prioritize criteria, there was a broad consensus about the most important criteria when carrying out MR3. Communication with the patient about the goal of MR3 and the current medication was perceived as very important. Different criteria were perceived as less important, like the price and expiry date of drugs. Small differences in prioritization were related to the experience of respondents with MR3, working in community pharmacy or nationality. Further refinement and research is needed, but the outcome of this study can act as a solid base for both community pharmacists and policymakers to assess the quality of MR3.

\section{PP004}

A survey of Irish community pharmacists to investigate the impact of COVID-19 on pharmacist well-being and pharmacy practice

\section{Aoife Fleming ${ }^{*}$ 1, 2 , Suzanne McCarthy ${ }^{1}$, Caoimhe Murphy ${ }^{1}$, Ciara Kenny ${ }^{1}$, Kieran Dalton ${ }^{1}$}

${ }^{1}$ Pharmaceutical Care Research Group, School of Pharmacy, University College Cork, ${ }^{2}$ Pharmacy Department, Mercy University Hospital, Cork, Ireland

Background and Objective: Community pharmacists have continued to provide medications and pharmacy services to their patients throughout the COVID-19 pandemic. The introduction of public health measures to curb the spread of COVID-19 has impacted on the delivery of pharmacy services and pharmacists have had to implement many changes to their practice. The primary objective of this study was to investigate the impact of the COVID-19 pandemic on pharmacist well-being and pharmacy practice in Ireland.

Method: A cross-sectional online survey (39 questions) was emailed to all registered community pharmacists $(n=3750)$ in Ireland in July 2020, remaining open for 5 weeks. The survey content was informed by an unpublished qualitative interview study by this research group on pharmacists experiences of working during the COVID-19 pandemic. Data from closed-ended questions were analysed in Microsoft ${ }^{\circledR}$ Excel and IBM ${ }^{\circledR}$ SPSS software version 24. Open comments underwent thematic analysis, facilitated by $\mathrm{NVivo}^{\circledR}$ software version 12. Ethical approval was obtained.

Main outcome measures: Community pharmacists' reported changes to well-being and work practices.
Results: A total of 564 surveys were received (response rate $=15 \%$ ). The majority of respondents experienced a workload increase (90.8\%). Most pharmacists (67\%) reported more difficulty interacting with patients and $46.3 \%$ felt less connected to their patients. Some reported an increase in job satisfaction (39\%) and most (62\%) agreed that their contribution had been appreciated and recognised more during the pandemic.

Most pharmacists reported a negative impact on their well-being (58.1\%), and $80.3 \%$ felt an increased risk of exhaustion or burnout at times. Most pharmacists (60.7\%) felt at increased risk of making dispensing errors, and experienced more ethical dilemmas since the pandemic began (56.3\%). The changes to pharmacy legislation were reported positively in terms of supporting patient adherence $(72.9 \%)$. Most believed that the introduction of a national electronic prescription transfer service improved communication with prescribers (73\%), and should continue after the pandemic (92.7\%). However, many pharmacists commented that pharmacist support was lacking during the initial months of the pandemic. Just over half (52.3\%) did not feel sufficiently protected, and $51.7 \%$ had a major concern about preparedness for a second wave of COVID-19 cases.

Conclusion: COVID-19 has had a significant effect on pharmacy practice in Ireland, with many community pharmacists experiencing a negative impact on their well-being. Supports to facilitate continuation of pharmacy services and to promote pharmacist well-being, and prevent burnout, are recommended going forward.

\section{PP005}

Chronic non-malignant pain patients' perspectives of the barriers and enablers of opioids therapy optimisation: study protocol

\section{Aziza Alenezi $^{*}{ }^{1}$, Vibhu Paudyal ${ }^{1}$, Asma Yahyouche $^{1}$ \\ ${ }^{1}$ School of Pharmacy, Institute of Clinical Sciences, University of Birmingham, Birmingham, United Kingdom}

Background and Objective: In the face of growing use of prescribed opioids, concern has arisen over opioid misuse and how patients may be supported in medication management.

Objective To explore the lived experience of adult patients receiving opioid therapy for relief from chronic non-malignant pain and the challenges faced.

Setting

UK based health website discussion forums.

Method: A search of two active, open-access forums were conducted to identify over two hundred discussion thread on managing chronic non-malignant pain with opioids. Relevant threads were coded using the Theoretical Domains Framework and analysed thematically.

Main outcome measures: Qualitative accounts of social and psychological factors enabling or impeding effective use, tapering and cession of opioids therapy for chronic non-malignant pain.

Results: Analysis identified four main themes from over two hundred relevant discussion threads: (1) the experience of living with chronic pain (2) facilitators of opioid therapy optimisation (3) barriers to opioid therapy optimisation (4) patients' perspective of pain management options. Patients experienced physical, social and psychological challenges, including social isolation, depression, anxiety and suicidal ideation. They struggled to retain a sense of independence and agency in the face of their "disabled" identity. Important facilitators of medication management were informal sources of information and coping strategies, doctor-patient relationship and support in the formally and community. Negative judgment by prescribers and lack of involvement in treatment decisions resulted in negative emotional consequences. 
Conclusion: Chronic non-malignant pain patients suffer challenges related to both the pain and medication management. Supportive relationships with providers are crucial in influencing emotional health and facilitating safe effective medication management.

References: Hewson C, Buchanan T, Brown I, et al Ethics guidelines for internet-mediated research. Leicester: British Psychological Society; 2013. https://www.bps.org.uk/sites/www.bps.org.uk/files/ Policy/Policy\%20-\%20Files/Ethics\% 20Guidelines\%20for\%20Internet-Mediated\%20Research\%20\%282013\%29.pdf

\section{PP007}

Community pharmacists' views on the implementation of a national electronic prescription transfer system to pharmacies

\section{Ciara Kenny $^{*}{ }^{1}$, Kieran Dalton ${ }^{1}$ \\ ${ }^{1}$ Pharmaceutical Care Research Group, School of Pharmacy, University College Cork, Cork, Ireland}

Background and Objective: To minimise COVID-19 transmission and ensure continued medication supply to patients in Ireland, legislation was implemented in April 2020 to permit electronic prescription transfer from prescribers directly to pharmacies using a national secure email system (Healthmail). With a clear need to assess how this initiative has impacted the delivery of patient care, the primary objective of this study was to evaluate community pharmacists' views of electronic prescription transfer to pharmacies via Healthmail.

Method: A cross-sectional online survey was disseminated via email in November 2020 to all Pharmaceutical Society of Ireland pharmacist registrants with 'community pharmacy' as their practice area $(n=$ 3,780). Closed-ended questions were analysed using Microsoft $^{\circledR}$ Excel and IBM ${ }^{\circledR}$ SPSS Statistics 26 . Respondents were provided with free text boxes to comment on how electronic prescription transfer could be improved and to provide any additional views; all comments underwent content analysis, facilitated by NVivo ${ }^{\circledR} 12$. Ethics approval was obtained prior to study commencement.

Main outcome measures: Community pharmacists' experiences of electronic prescription transfer via Healthmail and their views regarding its impact on patients and the implications for future practice.

Results: In total, 494 responses were received. At the time of the survey, $73.8 \%$ indicated at least $7 / 10$ prescriptions were sent via Healthmail. Most agreed it was an efficient and convenient method of prescription transfer $(89.7 \%$ ) and preferred receiving prescriptions in this way compared to other methods $(75.8 \%)$. Most had replied to a prescriber via Healthmail to conduct an intervention (90.7\%); of these, $81.9 \%$ agreed that they did not receive a response for some interventions and had to take further action. Although more than half of respondents felt that electronic prescription transfer via Healthmail added to their workload $(58.5 \%)$, most agreed that it had successfully integrated into dispensary workflow $(81.8 \%)$, that it should continue $(96.1 \%)$, and that it was a positive step for pharmacy practice $(94.7 \%)$. One of the key points from the open comment sections was the need to manage patient expectations regarding prescription preparation and the importance of having greater patient involvement going forward with electronic processes like this. Most pharmacists believed that patients did not have enough information about Healthmail (70.5\%), with $63.2 \%$ perceiving that it has led to patients feeling less involved in the decision-making related to their care. Furthermore, $46.1 \%$ indicated that Healthmail has had a negative/ strongly negative effect on patients' understanding and overview of their medication.
Conclusion: This study shows that pharmacists were mostly positive about the introduction of electronic prescription transfer to pharmacies in Ireland, but had important concerns regarding its negative impact on patients' medication management. If the use of electronic prescription transfer to pharmacies is to continue, further guidance is required for all stakeholders to achieve best practices with this process to optimise its integration in practice.

\section{PP008}

GEMED: results of a project to improve pharmaceutical care for nursing home residents

\section{Elisabeth Kretschmer* ${ }^{1}$, Diemut Strasser ${ }^{1}$, Regina Riedl ${ }^{2}$, Andrea Berghold ${ }^{2}$}

${ }^{1}$ Austrian Chamber of Pharmacists, Vienna, ${ }^{2}$ Institute for Medical Informatics, Statistics and Documentation, Medical University of Graz , Graz , Austria

Background and Objective: Nursing home residents have a high risk for adverse drug events (ADEs) due to polymedication and polymorbidity. Up to $40 \%$ of ADEs in frail elderly persons are assessed as preventable by an optimal use and an enhanced surveillance of drug therapy. [1]

Aim: to improve pharmaceutical care for nursing homes residents by reducing the risk for drug related problems (DRPs) with focus an ADEs.

Method: Prospective observational cohort study with two one-yearperiods in six community pharmacies and nursing homes;

Methods include continuous medicines reconciliation by community pharmacists according to the criteria of the medication appropriateness index with monthly meetings with nurses to identify ADEs due to changes in physical and mental status of the residents [2], and written information to the doctors about identified DRPs/ADEs with recommendations for changes in medication (classified corresponding to Allenet et al [3]).

Main outcome measures: number of residents with DRPs and ADEs Results: Period 1 (11/2016-10/2017): from 268 residents $(70.5 \%$ female, mean age: $82.2 \pm 10.4$ years, mean number of drugs/resident at the beginning of the period $(\mathrm{t} 1): 8.3+3.9$, mean number of drugs/ president at the end of the period (t2): $\overline{8} .5 \pm 3.8), 115$ (42.9\%) had $\geq 1$ DRP and $41(15.3 \%)$ residents had $\geq 1 \mathrm{ADE}$. In total, 249 DRPs were observed, including $21.7 \%$ ADEs, $19.3 \%$ inappropriate prescribing and $16.5 \%$ drug without indication. From 249 pharmaceutical recommendations addressed to the doctors (e.g. stopping a drug: $45.0 \%$, dose adjustment: $22.9 \%$, monitoring: $8.0 \%), 169(67.9 \%)$ were accepted.

Period $2(06 / 2018-05 / 2019)$ includes 209 residents $(67.5 \%$ female, mean age: $82.6 \pm 10.7$ years, mean number of drugs/resident $(\mathrm{t} 1)$ : 8.6+3.9, mean number of drugs/resident (t2): 8.5+3.9). In $92(44.0 \%)$ residents $\geq 1$ DRP occurred ( $\geq 1$ ADE: $21,10.0 \%)$. In total, 223 DRPs were observed, including $27.8 \%$ inappropriate prescribing, $17.5 \%$ drug without indication and $12.6 \%$ overdose. From 223 recommendations (e.g. stopping a drug: $41.3 \%$, dose adjustment: $21.1 \%$, monitoring: $13.9 \%), 130$ (58.3\%) were accepted.

Conclusion: regular medicines reconciliation can improve the quality of pharmaceutical care of community pharmacies for nursing homes. References: [1] Gurwitz J. et al. The incidence of adverse drug events in two large academic long-term care facilities. The American Journal of Medicine 2005; 118, 251-258

[2] Hanlon JT et al (1992) . A method for assessing drug therapy appropriateness. J Clin Epidemiol 1992; 45: 1045-1051 
[3] Allenet B. et al. Validation of an instrument for the documentation of clinical pharmacists' Interventions. Pharm World Sci (2006) 28:181-188

\section{PP009}

Take a breath: a community pharmacy service for smoking cessation

Ema Paulino* ${ }^{1}$, Catarina Ferreira ${ }^{1}$, Ana Pinto ${ }^{1}$, Mariana Rosa ${ }^{1}$, Luísa Teixeira ${ }^{1}$

${ }^{1}$ Ezfy, Lisbon, Portugal

Background and Objective: Community pharmacists are well positioned to offer products and services to aid smoking cessation. Through brief interventions, advice on the best treatment options, counselling on the correct use of smoking cessation products, and behavioural support, there is an opportunity to increase quit rates. We aimed to assess the feasibility and effectiveness of a smoking cessation service delivered by community pharmacists, with concurrent use of nicotine replacement therapy.

Design: A total of 110 pharmacies received training and educational resources to support the smoking cessation service, and formularies to collect data were designed to be used at each contact. A software system (Ezfy HealthCloud) was customized to be used by pharmacists conducting the service. Patients were enrolled in the programme following a successful initial brief intervention (opportunistic advice, discussion, negotiation and encouragement), to assess their motivation to quit smoking. Inclusion criteria included willingness to start the smoking cessation process using nicotine replacement therapy. After obtaining informed consent, patients had an initial consultation with the pharmacist to establish the smoking cessation plan, and were registered in the software system, which prospectively scheduled follow-up contacts on D-day (as agreed with the patient), one to two weeks after D-day, and one and two months following D-day. The service is still ongoing in pharmacies.

Results: Since July 2020 and as of June 2021, a total of 189 patients were enrolled in the programme, in 61 participating pharmacies. Data for gender was obtained for 182 patients, of which $100(52,9 \%)$ were male. The majority of patients $(n=54 ; 28,6 \%)$ were between $41-50$ years old. For a total of 150 patients where information at D-day was obtained, $142(94,0 \%)$ quit smoking on the agreed day, and 9 patients $(6,0 \%)$ re-scheduled D-day. As of June 2021, of the total number of patients enrolled, $56(29,6 \%)$ dropped-out of the programme, 15 $(7,9 \%)$ have had their first follow-up contact, $23(12,2 \%)$ their second follow-up contact, and $95(50,3 \%)$ have completed the programme. For those who have completed the programme, at 2 months, 69 participants $(72,6 \%)$ had sustained smoking abstinence.

Conclusion: Despite the observed attrition rate, the provision of a smoking cessation service by community pharmacists is feasible, and is effective in supporting people trying to stop smoking.

\section{PP010}

Challenges for mothers having G6PD deficient children in the United Arab Emirates: exploring a new horizon for the pharmacist educational role

Heba Mohamed* ${ }^{1}$, Lamia Al Hajri ${ }^{1}$, Amged Mustafa ${ }^{1}$ on behalf of Alia Hassan1, Meera Saleh1, Sara Ahmad1, Sara Walid1

${ }^{1} \mathrm{HCT}$, Dubai, United Arab Emirates
Background and Objective: Glucose 6-Phosphate Dehydrogenase (G6PD) deficiency, commonly known as fava bean anaemia or favism is a genetic disease that more often observed in male patients. The prevalence of G6PD deficiency is relatively high around the Mediterranean and Middle East; it is considered a main health challenge in a number of countries including the United Arab Emirates (UAE). Haemolysis is the most common medical problem in patients with G6PD deficiency, it can be triggered by consuming certain types of food, take certain drugs, or exposed to the stress of infection (Kasemy et al 2020). Avoiding the triggers and starting early treatments are very important to decrease the incidence of severe haemolysis (Glader et al. 2009). The study aims to explore the experience of mothers having G6PD deficient Children, their perception, challenges and coping strategies as well as to highlight new educational roles for community pharmacists in such conditions.

Method: A phenomenological design has been adopted using Semistructured online interview guided by a topic guide was carried out among eight participants in UAE, with different characteristics. Participants were recruited via purposive, snowball and convenience sampling. Interviews were conducted, audio recorded, transcribed and independently analyzed.

Main outcome measures: Outcomes included the identification of the main challenges faced by mothers having G6PD deficient Children in UAE and their perception towards the disorder. Shedding the lights on how pharmacists can play a crucial educational role to this community.

Results: Eight interviews (15-45 minutes each-via Zoom application) were conducted with mothers who have children with G6PD deficiency. The results covered two themes, the challenges faced by the mothers and their coping strategies, each theme contain six subthemes (food, medication, symptoms, emotional, information, and products). The results brought to light that half of the participants faced difficulties in obtaining accurate medical information about the condition, guidance on how to cope and facilitate their kids' lives. While other participants adapted smoothly with the situation and became knowledgeable about the condition with time. Most participants struggled to find accurate information and the proper food options.

Conclusion: Mothers with G6PD deficient Children seem to have various challenges and the major ones are deficiency in reliable information and support for G6PD children and their families. It is aimed that the findings of the study will shed the light on the disease and provide some recommendation for this population. Proper education programs are needed to ensure better awareness and better prevention of triggering factors that will lead to better health for children and families. The community pharmacists, the most available contact point to mothers, can lead these educational programs. Moreover, it was suggested to establishing a G6PD deficiency community to help mothers to connect and share their experiences. In addition, the study recommends having an application to help avoid some triggers especially from food and products.

References: Kasemy, Z. A., Bahbah, W. A., El Hefnawy, S. M., \& Alkalash, S. H. (2020). Prevalence of and mothers' knowledge, attitude and practice towards glucose-6-phosphate dehydrogenase deficiency among neonates with jaundice: a cross-sectional study. BMJ open, 10(2), e034079. https://doi.org/10.1136/bmjopen-2019034079

Harcke, S. J., Rizzolo , D., \& Harcke , H. T. (2019). G6PD deficiency. Journal of the American Academy of Physician Assistants, 32(11). doi: 10.1097/01.JAA.0000586304.65429.a7 


\section{PP011}

Clinical and organizational impacts of provenance of medical order (from hospital versus ambulatory setting) on patients and community pharmacy dispensing process: the prospective ORDHOSPIVILLE study

\section{Justine Clarenne* 1, 2, Julien Gravoulet $^{3,4}$, Virginie Chopard ${ }^{5}$, Julia Rouge $^{5}$, Amélie Lestrille ${ }^{1}$, François Dupuis ${ }^{3}$, Léa Aubert ${ }^{1,2}$, Sophie Malblanc ${ }^{5}$, Céline Mongaret ${ }^{1,2}$, Florian Slimano ${ }^{1,2}$ \\ ${ }^{1}$ Faculty of Pharmacy, Reims University, ${ }^{2}$ Department of Pharmacy, CHU Reims, Reims, ${ }^{3}$ Faculty of Pharmacy, Lorraine University, Vandoeuvre-Lès-Nancy, ${ }^{4}$ Pharmacie Gravoulet, Leyr, ${ }^{5}$ OMEDIT Grand Est, Agence régionale de santé, Nancy, France}

Background and Objective: Community pharmacists (CP) can detect and manage Drug-Related Problems (DRP) by performing pharmacist interventions (PI) during the dispensing process of medical orders (MO). MO from hospital could lead to additional DRP in the dispensing process for several reasons such as time of discharge from the hospital, prescribed drugs infrequently used or very expensive (mostly immediately unavailable in community pharmacy) and difficulties for calling prescribers. These DRP could have greater clinical and organizational impacts in comparison with MO from ambulatory setting, but this issue is not reported in the literature. The primary objective was to compare DRP detection and management with PIs performed by CPs whether MO are from hospital (MOH) or from ambulatory setting (MOA), respectively. The secondary objective was to assess the clinical and organizational impacts of these DRP on the patient's care pathway and CP workflow.

Method: Prospective study from January to June, 2020 promoted by two faculties of pharmacy and conducted by pharmacy students during their last 6-months internship in collaboration with their mentor CP. Each pharmacy student had to include $10 \mathrm{MOH}$ and 10 consecutive MOA. For each MO were collected: status/identification of prescriber and regulatory admissibility of MO. For each MO with at least one DRP detected were collected: type of DRP and PI by using the SFPC tool ${ }^{1}$; clinical and organizational impacts according to the CLEO tool ${ }^{2}$. The comparison between $\mathrm{MOH}$ and MOA groups was performed by Chi-square test with a significance level of alpha of 0.01 .

Main outcome measures: Frequency and type of DRP among MOH and MOA; clinical and organizational impacts between $\mathrm{MOH}$ and MOA when DRP detected.

Results: $\mathrm{N}=1174(51.5 \%) \mathrm{MOH}$ and $\mathrm{n}=1151$ (49.5\%) MOA were collected among $\mathrm{n}=120$ community pharmacies. $\mathrm{N}=114(9.71 \%)$ PIs were performed on $\mathrm{MOH}$ and $n=55(4.77 \%)$ on MOA. There was no difference in the DRP and PI subtype frequency depending on the MO setting. $\mathrm{N}=48(52.2 \%)$ hospital prescribers were "difficult" or "very difficult" to join. $\mathrm{MOH}$ were associated with a greater impact on time spent per MO than for MOA $(\mathrm{p}<0.001)$ and in twice as many cases with a greater proportion of unplanned consequences for patients, mainly delay in the initiation of a new medicine $(\mathrm{p}<0.001)$. The CP assessed the patient burden as minor in $86.1 \%$ and $81.0 \%$ for MOA and $\mathrm{MOH}$, respectively (NS).

Conclusion: Even if moderate impact, $\mathrm{MOH}$ are associated with more DRP detected and have greater clinical and organizational impacts on the patient's care pathway and CP workflow. Lack of communication between hospital and primary care and the amount of unavailable information are the two main reasons for these results. Implementation of clinical pharmacy activities at patient discharge as well as emergence of shared electronic health records between hospital and ambulatory settings could alleviate these impacts.

References: 1 Vo T-H, et al. Validation of a tool for reporting pharmacists' interventions in everyday community pharmacy, Journal of Clinical Pharmacy and Therapeutics 2018; 43(2): 240-248
2 Vo T-H, et al. CLEO: a multidimensional tool to assess clinical, economic and organisation impacts of pharmacists' interventions, European Journal of Hospital Pharmacy, 2021 (in press). DOI: 10.1136/ejhpharm-2020-002642

\section{PP012}

\section{Exploring community pharmacists' experiences during the} COVID-19 pandemic

\author{
Kieran Dalton $^{*}{ }^{1}$, Suzanne McCarthy ${ }^{1}$, Aoife Fleming ${ }^{1,2}$ \\ ${ }^{1}$ Pharmaceutical Care Research Group, School of Pharmacy, \\ University College Cork, ${ }^{2}$ Pharmacy Department, Mercy University \\ Hospital, Cork, Ireland
}

Background and Objective: Community pharmacists have remained on the frontline throughout the COVID-19 pandemic in order to ensure the continued safe provision of patient care. The primary objective of this study was to explore community pharmacists' experiences during this pandemic and their views on the changes to pharmacy practice.

Method: Semi-structured one-to-one interviews were conducted by the primary researcher via videoconference with community pharmacists from a variety of locations around Ireland, which were audiorecorded and subsequently transcribed verbatim. An interpretative phenomenological approach was used to guide the study design, topic guide development, and analysis of the interview transcripts to identify themes. ${ }^{1}$ This enabled the pharmacists' lived experiences, including the impact on their practice and their well-being, to be captured. Ethics approval was obtained.

Main outcome measures: Community pharmacists' lived experiences during the COVID-19 pandemic.

Results: Eight interviews were conducted (five female and three male; mean age of 37 years) between May and August 2020, with a median interview length of 61 minutes. Five main themes were identified:

1. Management of patient issues and behaviours - pharmacists had to demonstrate reassurance and assertiveness in addressing patients' fear and panic regarding medication supply, attempts at stockpiling, and compliance with public health measures.

2. Impact of infection control on communication and work processes - in-person communication was hindered by social distancing, masks, and physical barriers in the pharmacy; meanwhile, there was a surge in home medication delivery and communication via telephone, email, and online orders.

3. Supporting pharmacist resilience - pharmacists were fatigued and had limited outlets to distract from the pandemic, but knew they had to "get on with it" and praised the support from their colleagues and pharmacist peers.

4. Increased responsibility - the onus was on pharmacists to continually keep up to date with new information, and they felt significant responsibility for maintaining the safety and well-being of their patients and staff.

5. Recognising the contribution of community pharmacy - interviewees acknowledged the accessibility of community pharmacies compared to other healthcare settings. Pharmacists were proud of the profession in ensuring the continued provision of essential medication and advice, which they perceived was gratefully appreciated by the public.

Conclusion: This study provides an in-depth account of the extent to which community pharmacists adapted to the additional responsibilities placed on them during the COVID-19 pandemic. Further research is required to assess how this pandemic may affect 
pharmacists' well-being long term and the supports that are required, as well as to investigate how the communication changes impact on pharmacy practice and patient care in the future.

References:

1. Smith, J. A., \& Osborn, M. (2003). Interpretative phenomenological analysis. In J. A. Smith (Ed.), Qualitative psychology: A practical guide to research methods (p. 51-80). Sage Publications, Inc.

\section{PP013}

A toolkit of behaviour change techniques to support medication adherence in chronic disease

Isa B. Félix ${ }^{1}$, Cathal Cadogan ${ }^{2}$, Judith Strawbridge ${ }^{3}$, Afonso M. Cavaco $^{4}$, Marta M. Marques ${ }^{5,6}$, Mara P. Guerreiro" ${ }^{\text {1, } 7}$

${ }^{1}$ Nursing Research, Innovation and Development Centre of Lisbon (CIDNUR), Nursing School of Lisbon, Lisbon, Portugal, ${ }^{2}$ Trinity College Dublin, School of Pharmacy and Pharmaceutical Sciences, ${ }^{3}$ Royal College of Surgeons in Ireland, School of Pharmacy and Biomolecular Sciences, Dublin, Ireland, ${ }^{4}$ Faculty of Pharmacy, University of Lisbon, Lisbon, Portugal, ${ }^{5}$ ADAPT SFI Research Centre \& Trinity Centre for Practice and Healthcare Innovation, Trinity College Dublin, Dublin, Ireland, ${ }^{6}$ Comprehensive Health Research Centre (CHRC), NOVA Medical School, Lisbon, ${ }^{7}$ Centro de Investigação Interdisciplinar Egas Moniz (CiiEM), Instituto Universitário Egas Moniz, Monte de Caparica, Portugal

Background and Objective: Suboptimal medication adherence in chronic disease remains challenging and is associated with increased morbidity and mortality. Employing behaviour change techniques (BCTs) to target behavioural determinants could help to optimise medication adherence. This study aims to map standardised BCTs to determinants of non-adherence in persons living with chronic disease and provide examples of their operationalisation.

Method: Our starting point was a list of 21 core BCTs from an established taxonomy (BCTTv1) [1]. This list was previously derived from a literature review coupled with an expert panel [2], as part of the Train4Health project. We identified non-adherence determinants to chronic medication based on a literature search and then selected those both addressable by BCTs from our core list and amenable to change by health professionals. Determinants were categorised according to the capability - opportunity - motivation behaviour model (COM-B). We mapped BCTs to selected determinants through discussion within the multidisciplinary team (nursing, pharmacy, behavioural psychology). Finally, examples of BCTs operationalisation were derived.

Main outcome measures: Number of non-adherence determinants selected, BCTs per determinant.

Results: We selected three main determinants-forgetfulness, negative beliefs about necessity and concerns about medicinescategorised under psychological capability and reflective motivation (COM-B model), respectively. A total of five BCTs were mapped onto forgetfulness, including action planning (1.4), restructuring the physical environment (12.1) and adding objects to the environment (12.5). Planning to take a medicine at a particular time of the day, associated with a specific action (e.g. tooth brushing), is an operationalisation of "action planning". Six and five BCTs were mapped onto negative beliefs about necessity and concerns about medicines, respectively, four of which were shared between the two determinants (e.g. goal setting (1.1), feedback on outcomes of the behaviour (2.7)). BCTs can be operationalised in bundle (e.g. reaching an agreement about a goal for taking a medicine (1.1) and then discuss the presence or absence of side effects based on self-report (2.7)).
Conclusion: Our toolkit comprises standardised BCTs linked to nonadherence determinants in persons living with chronic disease, plus examples of operationalisation. The toolkit can facilitate adoption of BCTs in practice, which, in turn may enable better intervention tailoring whilst enhancing interventions' comprehensiveness and ensuring consistency of reporting. Additional work to manage medication adherence employs BCTs targeting health professionals, as part of multilevel interventions.

References: 1. Michie S, et al. The behavior change technique taxonomy (v1) of 93 hierarchically clustered techniques: Building an international consensus for the reporting of behavior change interventions. Ann Behav Med. 2013;46:81-95.

2. Guerreiro MP, et al. Development of a European competency framework for health and other professionals to support behaviour change in persons self-managing chronic disease. BMC Med Educ. 2021;1-14.

\section{PP014}

Polypharmacy in Bulgarian elderly patients with chronic conditions-a cross-sectional study

\section{Radiana Staynova* $^{1}$, Konstantina Lambreva ${ }^{2}$}

${ }^{1}$ Department of Pharmaceutical Sciences, Faculty of Pharmacy, Medical University of Plovdiv, Plovdiv, ${ }^{2}$ Multiprofile Hospital for Active Treatment Pazardzhik, Pazardhik, Bulgaria

Background and Objective: Elderly patients suffer from multiple chronic conditions, which makes them the major consumers of medications. The aim of this study was to assess elderly patients' attitudes towards prescribed medications and satisfaction with the provided pharmaceutical care services.

Method: A cross-sectional study was conducted between JanuaryMarch, 2021, using a self-administered questionnaire. The study setting were seven community pharmacies in Plovdiv and Pazardzhik, Bulgaria. The questionnaire was divided into two sections: demographic and main characteristics of patients (age, gender, number and type of chronic diseases and conditions, number of medications taken); patients' attitudes towards prescribed medications and satisfaction with the provided pharmaceutical care services. The second part of the survey included 10 statements based on a five-point Likert scale.

Main outcome measures: Number of chronic conditions and medications taken; The elderly patients' reported attitudes about prescribed medications and satisfaction with the provided pharmaceutical care service.

Results: The study included 104 geriatric patients (60 women and 44 men). The mean age of the respondents was $77.9 \pm 8.8$ years, with an age range from 65 years to 93 years. The mean number of chronic diseases suffered by each of the patients was 2.8 , including: hypertension $(79 \%)$, cardiovascular disease $(60 \%)$, diabetes $(44 \%)$, mental problems $(44 \%)$, etc. Less than half of respondents $(44 \%)$ reported taking 5-8 medications daily, followed by those taking more than 9 (33\%) and the remaining $23 \%$ noted taking 1-4 medications. The majority of patients $(64 \%)$ agreed or strongly agreed with the statement that they are taking too many medications. More than half of respondents $(58 \%)$ believed that taking too many medications affect negatively their daily activities. Sixty-two percent of study participants found it difficult to follow the instructions for taking the medication. Almost all participants $(93 \%)$ believed that the information provided by the pharmacist is useful (mean score $4.23 \pm 0.72$ ). The statement "I always ask the pharmacist when there is something I do not understand about my medication" received the highest mean score $(4.46 \pm 0.75)$. 
Conclusion: Medication consumption among elderly patients in Bulgaria is high. The assessment of elderly patients to the provided pharmaceutical care services is positive.

\section{PP015}

Patient adherence, satisfaction and counseling of diabetes medicine perceived by patients and their healthcare providers related to outcomes: insights from north Iraq

\section{Rawen Abdullah $^{*}$, Abdikarim M. Abdi ${ }^{2}$, Suha S. Shangula ${ }^{3}$, Bilgen Basgut ${ }^{4}$}

${ }^{1}$ Clinical Pharmacy, NEAR EAST UNIVERSITY, Nicosia, Cyprus, ${ }^{2}$ Clinical Pharmacy, Yeditepe University, Istanbul, Turkey, ${ }^{3} \mathrm{Clinical}$ Pharmacy, Hawler Medical University, Erbil, Iraq, ${ }^{4}$ Pharmacology, Baskent University, Ankara, Turkey

Background and Objective: Diabetes mellitus (DM) prevalence is continuously growing with around 1.4 million Iraqis have diabetes. Type $2 \mathrm{DM}$ prevalence is estimated to reach $13.9 \%$. Management of patients and outcomes varies across regions with factors such as counseling, medication related factors, Satisfaction and self-care activities being important. the aim is to assess quality of counseling information among DM patients receiving diabetic medications from physician and pharmacist, along with patient adherence to diabetic medications and the impact of counseling on clinical outcomes.

Design: A cross sectional, descriptive, face-to-face, closed ended, questionnaire study was conducted in Layla Qasim diabetic center and Ashty. Patient's demographic information was gathered along the Satisfaction with Information about Medicines Scale (SIMS), brief Morisky Medication Adherence Scale (MMAS) were utilized following validation of questionnaires.

Results: 150 patients accepted and were eligible to the study participation. Patients who participated in the study involved $52.3 \%$ males. Considering the Morisky scale, patients with high adherence showed a significantly lower HbAlc than non-adherent patient $(p<0.05)$. Patients were more satisfied with the action and usage of medications than potential side effects of the medications $(p<0.001)$. Conclusion: Patient adherence to their medications was suboptimal, non adherent patients had higher level of Hbalc and FBG comparing to adherent patients. Satisfaction of patients for indication and usage of anti-diabetic medications were higher than potential side effect.

\section{PP016}

Monitoring of adherence in community pharmacies with MEMS® and MARS-5 in patients taking DOAC

\section{Sara Desmaele ${ }^{*}$, Bernard Vrijens ${ }^{2,}{ }^{3}$, Koen Boussery ${ }^{4}$, Andreas Capiau $^{4}$, Maxim Grymonprez ${ }^{4}$, Lies Lahousse ${ }^{4}$, Els Mehuys ${ }^{4}$, Steurbaut Stephane ${ }^{5}$, Silas Rydant ${ }^{1,5,6}$ \\ ${ }^{1}$ Koninklijke Apothekersvereniging Antwerpen (KAVA), Antwerpen, ${ }^{2}$ Liège University, ${ }^{3}$ AARDEX Group, Liège, ${ }^{4}$ Pharmaceutical Care Unit, Faculty of Pharmaceutical Sciences, Ghent University, Ghent, ${ }^{5}$ Research group of Clinical Pharmacology and Clinical Pharmacy, Vrije Universiteit Brussel/UZ Brussel, Brussels, ${ }^{6}$ Laboratory of Physiopharmacology, Department of Pharmaceutical Sciences, University of Antwerp, Antwerpen, Belgium}

Background and Objective: Adherence to direct oral anticoagulants (DOAC) is crucial to prevent thrombosis or stroke, yet adherence remains suboptimal. A combination of various techniques like selfassessment and electronic monitoring is ideal to identify non-adherence, however not routinely implemented in daily practice.

Method: This interventional study was executed in 13 Belgian community pharmacies between October 2020 and April 2021. Patients on DOAC, apart from dabigatran, were recruited consecutively with a maximum of 15 participants per pharmacy. Participants received a Medication Event Monitoring system $\left(\mathrm{MEMS}^{\circledR}\right)$-cap, filled with DOAC for a period of 6 weeks. After this period, pharmacists read out the $\mathrm{MEMS}^{\circledR}$-cap and discussed the results with the patients. Subsequently, the MEMS ${ }^{\circledR}$-cap was refilled for another 6 weeks and the discussion with the patient was repeated 6 weeks later. Participants needed to complete the MARS-5 survey at every point of contact with their pharmacist: before the start, after 6 weeks and after 12 weeks.

Main outcome measures: Assessment and evolution over time of self-reported medication adherence and electronic adherence monitoring in real-life.

Results: A total of 158 patients were addressed, of which $56.0 \%$ met the inclusion criteria and agreed to participate (mean age: 70.4 years, $66 \%$ male). $79 \%$ of participants were one a once-daily dose (QD), $21 \%$ one a twice-daily dose (BID). The overall mean taking adherence for the QD regimen was $99 \%$ and $96 \%$ for the BID regimen based on the MEMS ${ }^{\circledR}$ data. The mean timing adherence in both groups was $86 \%$. The perceived adherence, based on the MARS-5, before the start of the study was $24.4 / 25$ and rose to $24.6 / 25$ after a period of 6 weeks $(n=76)$ and to $24.7 / 25$ after a period of 12 weeks $(n=65)$. Conclusion: Most patients stated they were adherent to therapy with a mean MARS-5-score of $24.4 / 25$ prior to the start of the study increasing to $24.7 / 25$ by the end of the study. This high level of adherence was confirmed by data from the MEMS ${ }^{\circledR}$-caps. Despite the limited number of participants, this study demonstrates the feasibility to combine various techniques to assess DOAC adherence by community pharmacies, although more research is needed to target the intervention to those that would benefit most from it.

\section{PP018}

The status of non-communicable diseases and drug related problems in a rural region of north Cyprus

\section{Servet Goksin ${ }^{*}{ }^{1}$, Abdikarim M. Abdi ${ }^{2}$, Loui Alsaloumi ${ }^{1}$, Bilgen Basgut $^{3}$}

${ }^{1}$ Clinical Pharmacy, NEAR EAST UNIVERSITY, Nicosia, Cyprus, ${ }^{2}$ Clinical Pharmacy, Yeditepe University, Istanbul, ${ }^{3}$ Pharmacology, Baskent University, Ankara, Turkey

Background and Objective: Non-communicable diseases (NCDs) are the leading cause of death globally with high prevalence in North Cyprus and Turkey. Despite multiple initiatives led by the state and other stakeholders for the last two decades, the burden of chronic disease continues to increase rapidly with lower resource areas being affected the most. The aim of this study is to evaluate the status of care of NCD patients in a rural area in North Cyprus.

Design: A Cross-sectional study was carried involving patients with one or more NCD visiting a community pharmacy in Dilikaya village in North Cyprus. Patient's demographics, beliefs about medicine, lab values adherence and quality of life were evaluated using validated tools. A clinical pharmacist assessed patient's status in regards to goals of therapy achievement, identified drug related problems (DRPs), and categorized DRPs using PCNE tool version 9.2.

Results: Responders were 97 out of 200 that were eligible and approached to participate in the study ( $48.5 \%$ response rate). Median age of responders was $62(\mathrm{IQR}=15)$ years while Females formed 
$58.8 \%$. Only $54 \%$ of hypertension patients reached target blood pressure. More than $40 \%$ of T2DM patients fail to achieve target $\mathrm{HbA1c}$ level within an average duration of therapy of 10 (IQR=9.25) years for the studied patient group. Most patients (71\%) with Hypertension DM or CAD were not compliant in their lifestyle and diet and had a DRP $(86.6 \% ; 2.1$ DRP per patient). Insufficient dosing and inappropriate indication for a drug were the DRPs significantly impacting target achievement while Inappropriate drugs according to guidelines was the DRP type mostly affecting quality of life of the studied patients. Other factors associated with worse QoL and lower EQ-5d scores include female gender unemployment status, and belief "that Medicines do more harm than good",

Conclusion: Drug related problems and non-adherence were prevalent in this study. Almost one half of hypertensive patients were reported not to achieve target blood pressure, while only one fifth of patients with T2DM were found to achieve diabetes therapy targets. Community pharmacist are well suited in identifying therapy gaps and form an opportunity to improve outcomes in the management of chronic disease in rural areas.

\section{PP019}

What do we learn about the treatment and management of gout from listening to patients?

\section{Silas Rydant ${ }^{*}$ 1, 2, 3 , Ellen Jacobs ${ }^{4}$, Astrid Verreth ${ }^{4}$, Wim Martinet $^{2}$, Charlotte Bekker ${ }^{5}$, Guido De Meyer ${ }^{2}$, Bart Van den Bemt $^{5}$, Hans De Loof ${ }^{2}$}

${ }^{1}$ Research group of Clinical Pharmacology and Clinical Pharmacy, Vrije Universiteit Brussel/UZ Brussel, Brussels, ${ }^{2}$ Laboratory of Physiopharmacology, Department of Pharmaceutical Sciences, University of Antwerp, ${ }^{3}$ Koninklijke Apothekersvereniging Antwerpen (KAVA), ${ }^{4}$ University of Antwerp, Antwerpen, Belgium, ${ }^{5}$ Department of Pharmacy, Radboud university medical center, Nijmegen, Netherlands

Background and Objective: Gout is the most prevalent arthritis that causes inflammation, pain and disability. Despite effective treatment options and international guidelines, its treatment remains suboptimal. This study aims to identify the lived experiences of patients with gout, their insights about their therapy and their relationships with caregivers.

Method: A qualitative study with individual semi-structured interviews was conducted between February 2021 and May 2021. Adult patients with confirmed gout were interviewed about their perception, knowledge and experiences about their diagnosis, the initiation and continuation of urine-lowering therapy (ULT), how they handle gout attacks and lastly, their relationship with their physicians and pharmacists. Interviews were transcribed verbatim and analysed thematically using NVivo.

Main outcome measures: Outcomes included the perception, knowledge and experiences of patients that are being treated for gout. Results: In total, 22 patients were interviewed ( $82 \%$ male, mean age 64 years). Although almost all patients stated that they are therapy compliant, their accounts revealed a remarkable lack of knowledge about the disease, treatment and treatment goals. All participants had a family physician, but participants mention that gout and its treatment is rarely discussed after first diagnosis and initiation of treatment. Occasionally, treatment was even hampered by a strained relationship with their primary care physician. Patients experience the same lack of interest and follow-up when visiting their pharmacist and rather feel that the illness is not prioritized by both their physician as well as their pharmacist. Most patients state they are compliant to therapy and reported that their urine-lowering therapy (ULT) remained unchanged since initiation. Only one participant remembered a step-up treatment and only one patient was prescribed prophylactic anti-inflammatory drugs during initiation. Patients also reported self-medication during a gout attack, but conceded errors were made in the process due to unclear or even incorrect instructions.

Conclusion: Most patients state they are compliant to therapy, but their understanding of gout treatment is poor and they experience a lack of interest from their caregivers. Errors in self-medication when using anti-inflammatory drugs occur due to unclear instructions and patients report that ULT has not been initiated like guidelines prescribe with recurring gout attacks as a consequence. Our study demonstrates the need for a higher sense of urgency about gout among first-line physicians and pharmacists. Furthermore, this study reinforces the need for patient education and interprofessional training and collaboration between caregivers.

\section{PP020}

Investigation of drug interactions and patient profile with asthma in a community pharmacy

Nurdan Yaban ${ }^{1}$, Songül Tezcan*1

${ }^{1}$ Marmara University Pharmacy Faculty, Istanbul, Turkey

Background and Objective: To investigate the drug interactions and patients' profile of the patients with asthma.

Method: This was a retrospective and descriptive study conducted in a community pharmacy in Turkey. The prescriptions of the patients were investigated between January-December 2020. The patient profile and drug list of the patients were recorded. Drug interactions were analyzed via Medscape drug interaction checker and Lexicomp database.

Main outcome measures: Determination of patient's sociodemographic characteristics and drug interactions.

Results: Of 54 patients $33(61 \%)$ were female and the mean age was 54. Most of the patients $(76 \%)$ had at least one comorbid disease (mostly vasomotor rhinitis, $\mathrm{n}=19$, and hypertension, $\mathrm{n}=17$ ). While $54 \%$ of the patients were prescribed Montelukast for asthma treatment, $36 \%$ of the patients were prescribed hydrochlorothiazide for hypertension treatment. While a total of 89 drug interactions were determined using via Medscape database, it was found that $87 \%$ of them were classified as "monitor closely". On the other hand, 128 drug interactions were determined in Lexicomp and $76 \%$ of them were in the " $C$ " category.

Conclusion: The number of comorbid diseases was found to be higher in the patients. Lexicomp database was found to give higher number of drug interactions compared to Medscape drug interaction checker. We think that clinical pharmacists have an important role in determining the patient profile and drug interactions in asthma patients in community pharmacy settings.

\section{PP021}

Investigation of drug interactions and patient profile with chronic obstructive pulmonary disease in a community pharmacy

\author{
Nurdan Yaban ${ }^{1}$, Songül Tezcan* 1 \\ ${ }^{1}$ Marmara University Pharmacy Faculty, Istanbul, Turkey
}

Background and Objective: To determine the drug interactions and patients' profile of the patients with chronic obstructive pulmonary disease (COPD). 
Method: This was a retrospective and descriptive study conducted in a community pharmacy in Turkey. The prescriptions of the patients were investigated between January and December 2020. The patient profile and drug list of the patients were recorded. Drug interactions were analyzed via Medscape drug interaction checker and Lexicomp database.

Main outcome measures: Determination of patient's sociodemographic characteristics and drug interactions.

Results: Of 42 patients $31(74 \%)$ were male and the mean age was 69 . Most of the patients $(83 \%)$ had at least one comorbid disease (hypertension, $\mathrm{n}=25$ ). While $55 \%$ of the patients were prescribed Fluticasone for COPD treatment, $95 \%$ of the patients were prescribed antihypertensive drugs. While a total of 199 drug interactions were determined using via Medscape database, it was found that $78 \%$ of them were classified as "monitor closely". Similarly, 208 drug interactions were determined in Lexicomp and $76 \%$ of them were in the "C" category.

Conclusion: The mean age of the patients was higher than 65 years and the number of comorbid diseases was found to be high. Additionally, the investigation of number of drug interactions was found to be similar in the two databases. We think that clinical pharmacists have an important role in determining the patient profile and drug interactions in COPD patients in community pharmacy settings.

\section{PP022}

Psychometric properties of the Turkish version of the family caregiver medication administration hassles scale

Zehra Betül Kıngır* ${ }^{*}$, Mesut Sancar ${ }^{2}$, Refik Demirtunç ${ }^{3}$, Çağatay Nuhoğlư $^{3}$, Cemile H. Mısırlı ${ }^{3}$, Betül Okuyan²

${ }^{1}$ Uskudar University, ${ }^{2}$ Marmara University, ${ }^{3}$ University of Health Science, İstanbul, Turkey

Background and Objective: The aim of this study was to evaluate the validity and reliability of Turkish version of The Family Caregiver Medication Administration Hassles Scale (TR-FCMAHS) (1).

Design: This methodological study has been conducted in community pharmacies $(n=3)$ in Istanbul, Turkey for five months in 2020. After appropriate "forward-backward" translation, content validity was assessed during pilot study $(\mathrm{n}=20)$.

Test-retest reliability was assessed within 2 weeks. Kaiser-MeyerOlkin Measure of Sampling Adequacy and the Bartlett's Test of Sphericity were assessed and varimax rotation was used for factor analysis. Internal consistency was assessed by using Cronbach's alpha. Criterion validation was evaluated by using Spearman correlation test between the scores of TR-FCMAHS and validated Turkish version of the Zarit Caregiver Burden Interview (TR-ZBI) (2).

Results: Among 314 family caregivers, the mean age [standard deviation] was 48.1[13.5] and 68.5 was female. The mean of age of the patients was 62.8[14.3]. The mean score of TR-ZBI was 30.2[9.2]. Most of them (94.9\%) had high caregiver burden based on the score of TR-ZBI. The mean score of TR-FCMAHS was 41.3[12.6]. The test-retest reliability of the scale $(\mathrm{n}=30)$ was high $(\mathrm{r}=0.868 ; p<0.001)$. Kaiser-Meyer-Olkin Measure of Sampling Adequacy yielded a value of 0.794 and the Bartlett's Test of Sphericity was significant $(p<0.001)$. Factor analysis determined six subscales which explained $62.0 \%$ of the total variance. The Cronbach's alpha was found 0.848 for total scale. Spearman's correlation coefficient was 0.472 between the score of TR-FCMAHS and TR-ZBI $(p<0.01)$.

Conclusion: TR-FCMAHS could be used to assess Turkish family caregiver medication administration hassles.

Keywords: Family Caregiver, Medication Administration Hassles, Clinical Pharmacist.

\section{References:}

1. Kao HFS. Medication administration hassles for Mexican American family caregivers of older adults. Nursing and Health Sciences 13(2):133-40.

2. Babacan Yıldız G, Yacı Ö, Akça Z, Kulaksızoğlu IB. Reliability and Validity of the Abbreviated Turkish Version of the Zarit Caregiver Burden Interview. Bezmialem Science 2016; 2: 60-4.

\section{PP023}

Patient knowledge, beliefs, experiences and preferences for complementary and alternative medicine (CAM) for selfmanagement of diabetes: a qualitative study using patient online forums

\section{Abdulaziz Alzahrani* $^{1}$, Sheila Greenfield ${ }^{2}$, Vibhu Paudyal ${ }^{1}$ \\ ${ }^{1}$ Institute of Clinical Sciences, ${ }^{2}$ Institute of Applied Health Research, University of Birmingham, Birmingham, United Kingdom}

Background and Objective: Global diabetes burden reached 463 million cases in 2019. Clinical pharmacists can have an important role in optimising treatment plans, goals and outcomes but need to be aware of prescribed, as well as non-prescribed treatments patients use for self-care. One way patients exchange health information and share experiences and provide advice to one another is through online forums and discussion boards. This study aimed to explore patients' beliefs, experiences and preferences in relation to their use of complementary and alternative medicine (CAM) for diabetes through the use of data from online patient forum discussions.

Method: This qualitative study was conducted by performing an internet search using Google search engine to identify relevant online forums focussing on CAM use in diabetes. Search terms were compiled from the results of our recent systematic review of global prevalence of CAM use amongst adults with diabetes. Only blogs in forums for which no membership was required for access were included in this study as data are publicly available in public domains Main outcome measures: Patient perspectives regarding CAM use in diabetes

Results: A total of 22 relevant online forums were identified, containing 77 threads and 1156 replies. Data analysis suggested that side effects of modern medicines, reduce insulin need, reduces stress hormones to allow the body to more readily absorb / use the insulin and the belief that CAM can reverse diabetes were key factors related to patient use of CAM. Further analysis is being undertaken and the final results will be available by the conference date.

Conclusion: Patients share important information about diabetes knowledge and management in on line blogs. Some beliefs and advice may adversely affect patient adherence, behaviour and disease outcomes. Clinical pharmacists are ideally placed to initiate conversations about these topics and work with patients to advise, support adherence and identify the risks of interactions and adverse effects when CAMs are used in conjunction with prescribed treatments.

\section{PP025}

Developing a competency educational training framework to support pharmacists managing long-term conditions

\section{Laura Moura $^{*}{ }^{1}$, Filipa A. da Costa ${ }^{1}$, Sotiris Antoniou ${ }^{2}$}

${ }^{1}$ Faculty of Pharmacy, University of Lisbon, Lisbon, Portugal, ${ }^{2}$ St. Bartholomew's Hospital, London, United Kingdom 
Background and Objective: The International Pharmaceutical Federation (FIP) identified competency-based practice as one of the key areas of development for the pharmacy profession.

Long-term conditions (LTC) affect a considerable proportion of the population. Therefore, preparing pharmacists working in primary care to better manage LTC is crucial.

In the UK, there have been an increasingly investment on pharmacists working in primary care. Although some education training exists, specific LTC management competency education is lacking.

The primary aim of this project is to develop a competency educational training framework to support pharmacists working in GP practices managing LTC. The secondary aim is to test this framework for Atrial Fibrillation (AF) as proof of concept.

Design: Miller's pyramid for assessing clinical competence was considered to identify the most suitable teaching and assessment methods for each level of competence development. A before and after assessment model was considered with multiple assessment moments to evaluate immediate impact and sustainability of training. The content of the training takes stock of Merrill's Instructional Design Principles. Bloom's Taxonomy was used to define learning outcomes.

For AF, the educational package also engages on three key elements of the AF pathway, identified by the Academic Health Science Networks: detection, protection, and perfection (DPP).

FIP Global Competency Framework for Pharmacists and Royal Pharmaceutical Society Foundation Pharmacy Framework have been considered and linked throughout development process, guaranteeing the alignment with both international and national orientations.

Results: An educational package was developed. Prior to the first training modules, initial assessment will be conducted to evaluate baseline knowledge (multiple-choice questionnaire, MCQs), selfconfidence and outcomes at patient level (e.g. proportion of patients eligible for treatment receiving first-line therapy). These assessments will be repeated at 2 months and at 9 months.

The training envisaged comprises five modules going through the DPP concept using a theory-based approach. This will be followed by practical training, which includes case resolution and discussion, simulation (evaluated through OSCEs), real-world clinical practice (evaluated through remote observation of clinical consultations) and reflective practice. Practice-based training will be continued during 6 months before the sustainability final assessment to allow for practices with lower patient inflow to gather enough experience in managing AF.

Conclusion: The framework is now being implemented as a pilot project, training approximately 25 pharmacists for managing AF in GP practices located in London. It is expected that the developed framework will contribute to improve primary care pharmacists' capacity to manage LTCs.

\section{PP026}

Designing a theory-driven, evidence-based and user-centred web application to simulate behaviour change support in persons with chronic disease

DUARTE Taranta ${ }^{1}$, RICARDO Santos ${ }^{1}$, ANA P. Cláudio ${ }^{2}$, MARIA B. Carmo ${ }^{2}$, GREGOR Stiglic ${ }^{3}$, LUÍS Correia ${ }^{2}$, NINO Fijacko $^{3}$, ISA B. Félix ${ }^{4}$, ANASTASIYA Barabash ${ }^{5}$, MARGARIDA Henriques ${ }^{5}$, MUSTAFA Abacioglu', MARA P. Guerreiro* $^{*}$

${ }^{1}$ Faculdade de Ciências da Universidade de Lisboa, Lisbon, Portugal, ${ }^{2}$ LASIGE, Faculdade de Ciências da Universidade de Lisboa, Lisbon, Portugal, ${ }^{3}$ University of Maribor, Maribor, Slovenia, ${ }^{4}$ CIDNUR,
${ }^{5}$ Escola Superior de Enfermagem de Lisboa, Lisbon, Portugal, ${ }^{6}$ Marmara University, Istanbul, Turkey

Background and Objective: Healthcare and other professionals are expected to support behaviour change for the self-management of chronic disease [1]. Students present a skills gap in behaviour change support, resulting from curricular insufficiencies and limited training opportunities. The Train4Health project addresses this challenge through an innovative educational package. This paper reports the design of a web-based application for training behaviour change support.

Design: Design was grounded in a literature search, co-creation with users and requirements prioritisation. Prior project work informing software design includes an interprofessional European competency framework to support behaviour change in chronic disease [1], a list of core behaviour change techniques (BCTTv.1) [1], a learning outcomes-based curriculum derived from the framework and case studies. The literature was searched to retrieve evidence on design elements and components. Twelve international focus groups were conducted with students and educators from pharmacy, nursing and sport sciences. Requirements were prioritised through the Moscow approach.

Results: The use of virtual patient simulation and gamification appear promising in health professionals' education [2,3]. The self-determination theory enables an understanding of how gamification may enhance engagement and motivation, whilst helping avoid pitfalls in implementation [4]. Requirements elicited from focus groups (e.g. gamification, feedback) and team discussion were prioritised into "must have", "should have" and "won't have". A proof-of-concept web-based application was developed, in which users will interact with 2D virtual humans (VHs) playing the role of persons living with chronic disease. Each of the four case studies offers training in brief and long interventions. VHs communicate with users through a synthetic voice and facial expressions; input consists of buttons depicting two options. The choice of the less correct option prompts immediate feedback within each session. Once the session is finished, users receive feedback plus a point system providing insight about performance and are directed to self-debriefing, via Gibbs' reflective cycle. Implemented gamification strategies include an acknowledgement system (e.g. badges).

Conclusion: The design of the web-based application includes simulation with $2 \mathrm{D}$ virtual humans and gamification, underpinned by the self-determination theory, plus components such as feedback and selfdebriefing. Development is on-going and the application is being tested iteratively with students and educators.

1.Guerreiro et al (2021) doi.org/10.1186/s12909-021-02720-w

2.Kononowicz et al (2019) doi: 10.2196/14676

3.Gentry et al (2019) Doi: 10.2196/12994

4.Rutledge et al (2018) doi: 10.1097/ACM.0000000000002183

\section{PP027}

Assessment of knowledge of community pharmacists on cancer and cancer screening methods

\author{
Ayşen Uygun ${ }^{1}$, Songül Tezcan* ${ }^{2}$, Nazlı D. Calıskan ${ }^{1}$ \\ ${ }^{1}$ Marmara University Institute of Health Sciences, ${ }^{2}$ Marmara \\ University Pharmacy Faculty, Istanbul, Turkey
}

Background and Objective: The aim of the study is to evaluate the level of knowledge of community pharmacists about cancer and screening methods.

Method: This study was a descriptive cross-sectional study and was conducted between January and June 2020 in Istanbul at Turkey. Profile record form and a structured questionnaire was applied to 
pharmacists as online. The questionnaire was prepared by the researchers and consisted of 49 questions; basic information about cancer (15 items), signs and symptoms of cancer (11 items), causes of cancer and risk factors (14 items), cancer screening methods ( 9 items).

Main outcome measures: Assessment of community pharmacists' knowledge about cancer and screening methods.

Results: Of 133 pharmacists 99 were female and $51 \%$ were served for $0-15$ years. It was determined that the majority $(>90 \%)$ of the pharmacists included in the study did not receive any education on cancer and/or cancer screening methods after graduation. The sufficient knowledge level of the pharmacists about cancer basics, signs and symptoms of cancer and risk factors of cancer were found to be as $80 \%, 77 \%$ and $67 \%$, respectively. Internal consistency level of the questionnaire was calculated (Cronbach's alpha $=0.814$ ). The item of the breast cancer screening methods "Every woman should perform breast self-examination and notify a health professional when there is a change in breast appearance or feeling," was answered correctly by $98 \%$ of the pharmacists. It was determined that $82 \%$ of the pharmacists gave the correct answer to the item of the cervical cancer screening methods "Women aged 21-30 years should have a PAP smear every 3 years".

Conclusion: According to the results of the study, while the rate of sufficient knowledge level on cancer was found to be low, knowledge on the cancer screening was found to be sufficient. We think that pharmacists play an important role in increasing the knowledge of both their colleagues and patients and it is important for the pharmacists should receive training on cancer and screening methods and to follow the guidelines closely and to internalize continuous education.

\section{PP028}

Exploring types of medication errors and contributing factors associated with direct-acting oral anticoagulants

\section{Abdulrhman Alrowily $^{*}$ 1, 2 , Zahraa Jalal ${ }^{2}$, Vibhu Paudyal ${ }^{2}$ \\ ${ }^{1}$ Pharmaceutical Department, King Fahad Military Medical Complex , Dhaharan, Saudi Arabia, ${ }^{2}$ School of Pharmacy, University of Birmingham, Birmingham, United Kingdom}

Background and Objective: Direct-acting oral anticoagulants (DOACs) have been in clinical practice since 2008 for treatment and prevention of recurrent thromboembolic events such as pulmonary embolism and deep venous thrombosis [1,2]. Previous reports indicated their increasing clinical use [3], but little is known about incidence of errors. This study aimed to explore the type of the reported medication errors and identify the most frequently contributing factors associated with DOACs medication errors.

Method: The study used databases of medication errors reported during the period from January 2008 to December 2020 from a national regulatory body and two tertiary care hospitals in Saudi Arabia. Statistical analyses were performed using IBM (SPSS) Statistics Version 24.0 software programs.

Main outcome measures: Types, Subtype errors and contributing factors associated with DOACs in Saudi Arabia.

Results: The database revealed a total of 199 medication error incidents related to DOAC from January 2008 to December 2020. The average patients age was 63.5, ranging from 2 to 96 years old with equal gender distribution. The average reported weight was 75 , ranging from 29 to $166 \mathrm{Kg}$. The average reported duration of treatment when incidence happened was 90 days with a very wide range from one day to one year. The majority of the sample $(94.5 \%)$ showed other comorbidities rather than the disease of the indicated DOAC drug. Apixaban was the most frequent drug associated with medication error with $134(67.3 \%)$ incidents, followed by rivaroxaban $(18.6 \%)$ and dabigatran $(14.1 \%)$. Prescribing errors were the most common representing $81.4 \%$, followed by dispensing, administration and monitoring errors $10.6 \%, 4.5 \%$, and $2.0 \%$, respectively. Polypharmacy, indication of treatment, duration of therapy, and weight were the most significant contributing factors associated with errors.

Conclusion: This observational study considered the first of its type investigating DOACs errors in Saudi Arabia. Developing risk preventive or reduction strategies at national level using the expertise of clinical pharmacists is very important in reducing occurrence of medication errors with DOACs that may lead to serious adverse events such as bleeding.

References:1. National Institute for Health and Care Excellence. Atrial Fibirlliation: management Available at: https://www.nice.org.uk/guidance/cg180. Accessed 10 Mar 2021

2. Henriksen J, Nielsen L, Hellebek A et al (2017). Medication errors involving anticoagulants: data from the Danish patient safety database. Pharmacol Res \& Perspect 5(3): p. e00307

3. Rahmanzade, R., Diaz, F. C., Zaugg, et al (2020). Therapeutic duplication of anticoagulants: a retrospective study of frequency and consequences in a tertiary referral hospital. Thrombosis Journal, 18(1), 1-9

\section{PP029}

To what extent are health care professionals in English hospitals aware of the World Health Organization's medication safety challenge?

\section{Aikaterini Triantafyllou* ${ }^{1}$, Sara Garfield ${ }^{1}$, Sakshi Shastri ${ }^{2}$, Bryony D. Franklin ${ }^{2}$, Bridget Coleman ${ }^{3}$}

${ }^{1}$ Imperial College Healthcare NHS Trust, ${ }^{2}$ UCL School of Pharmacy, ${ }^{3}$ Whittington Health NHS Hospital, London, United Kingdom

Background and Objective: This study aimed to evaluate healthcare professionals' awareness of the World Health Organization's (WHO) Third Global Patient Safety Challenge ${ }^{[1]}$ and their knowledge of its focus areas (main domains/priority areas), in a sample of London hospital organizations.

Method: A descriptive and cross-sectional study was carried out at two London hospital organizations in 2018/2019. The data were collected using a validated questionnaire developed by the researchers in order to evaluate awareness and knowledge about the challenge. A purposive sampling strategy was followed to recruit doctors, nurses and pharmacists. The data were analysed using descriptive statistics; chi-square and t-tests were used to test for associations between the different professions and their awareness/knowledge.

Main outcome measures: The goal of the study was to investigate the awareness and knowledge of the challenge within doctors, pharmacists, and nurses and to make comparisons among different groups of professionals and hospital organizations.

Results: Two hundred and twenty healthcare professionals participated. The majority of participants were not aware of the challenge. Awareness of the challenge was significantly higher at Hospital 2 than Hospital 1. However, participants at Hospital 1 had significantly more knowledge of the challenge's priority areas, main domains, goal, and five-point plan than Hospital 2. There was a non-significant trend of nurses and pharmacists having more knowledge than doctors.

Conclusion: This study showed that healthcare professionals' knowledge about the challenge was limited. There is a need to raise awareness. The creation of a new tool (checklist) may help promote the challenge. 
References: 1 . World Health Organization The third Global Patient Safety Challenge. [Online]. Available from: https:// www.who.int/patientsafety/medication-safety/en/[Accessed 11 June 2021].

\section{PP030}

Small datasets as a source of change: use of albumin in clinical context

\section{Ana Rita Lemos ${ }^{*}$, Pedro Cardoso ${ }^{1}$, Miriam Capoulas ${ }^{1}$, Cláudia Santos ${ }^{1}$ \\ ${ }^{1}$ Hospital da Luz Lisboa, Lisbon, Portugal}

Background and Objective: Albumin clinical usage implies a careful analysis due to its risks, since it is a human plasma-derived product, and its high cost for the patient. Additionally, blood and plasma-derived donations are decreasing, especially during SARSCoV-2 pandemic context, reinforcing the importance of these medicines rational use. Application of healthcare data has allowed to increase medicines rational use and to benefit the patients. Small datasets are progressively used in association with more advanced prediction models permitting the development of robust analysis and predictions. The study goals were to analyze the prescription and the outcomes of Human Albumin treatment, using a database built in the Pharmaceutical Services, to establish new internal guidelines based on the results found and, to prepare data for machine learning.

Design: Patients who received Human Albumin at the Hospital da Luz Lisboa between January of 2020 and February of 2021 were retrospectively analyzed regarding the clinical context based on the clinical process (diagnosis, analytical evolution).

Results: Thirty eight prescriptions were analyzed. The median duration treatment was 2 days and the most prescribed daily dose was $30 \mathrm{~g}$. The majority of the prescriptions were made in the context of hypoalbuminaemia, followed by paracentesis. Patients with albumin prescription due to hypoalbuminaemia, mean albuminaeamia was $1.63 \mathrm{~g} / \mathrm{dL}$. The response rate in these patients was $92 \%$, with an increase of average albumin of $0.58 \mathrm{~g} / \mathrm{dL}$, being a significant outcome $(\mathrm{p}<0.01)$. Low correlation between the number of treatment days and the increase in the Albumin value was observed.

Conclusion: All prescriptions were among recommended therapeutic indications. However, it seems there is no defined criterion regarding the Albumin level that establishes the need to be treated and the dose administrated for each indication. Although the effective outcome has been significant, it would be relevant to assess the treatment duration, indication and outcomes in future studies to develop methodologies optimizing Albumin use. This analysis thus establishes the basis to apply a machine learning model that allows to measure whether the prescribed dose will provide the best therapeutic outcome possible for the patient.

\section{PP031}

Clinical pharmacist in the emergency department during nighttime hours

Astrid Heeremans ${ }^{*}{ }^{1}$, Saskia Van Kemseke ${ }^{1,2,3}$, Ellen Oudaert ${ }^{1,2}$, 3 , Pieter-Jan Cortoos ${ }^{1}$, Ives Hubloue ${ }^{2,3}$

${ }^{1}$ Pharmacy Department, ${ }^{2}$ Emergency Department, UZ Brussel, ${ }^{3}$ Research Group on Emergency and Disaster Medicine, Vrije Universiteit Brussel, Brussels, Belgium
Background and Objective: The Emergency Department (ED) is a 24/7 high-risk setting for medication discrepancies (MDs) and drugrelated problems (DRPs). Presence of a clinical pharmacist (CP) in the ED can improve medication safety by performing structured medication reconciliation (MR) and review. The objective was to identify the importance of a $24 / 7 \mathrm{CP}$ in the ED by determining the number and type of MDs and DRPs after performing MR and review simulated at nighttime hours $v s$. $<24 \mathrm{~h}$ after ED admission.

Method: This prospective, interventional study was conducted between $01 / 2021$ and $02 / 2021$ in a tertiary care university hospital in Brussels, Belgium. Patients $\geq 60$ years with $\geq 4$ chronic medications admitted in the ED (5 p.m.-7 a.m., from Sunday night to Friday morning) and a signed informed consent were included. MR and review were simulated as if these were performed at nighttime hours and were completed $<24 \mathrm{~h}$ after ED admission by contacting the community pharmacist and general practitioner.

Main outcome measures: MDs, DRPs and DRP severity were recorded for the chronic therapy and prescribed medication. Physicians' acceptance rate for pharmaceutical recommendations (PIs) was assessed.

Results: During 30 days, 206 patients were included. A statistically significant difference in median number of chronic medications was discovered between MR by the $\mathrm{CP}$ and patient file, both at nighttime hours (9.0 [IQR 7.0-12.0] vs. 8.0 [IQR 6.0-11.0]; $\mathrm{P}<0.005$ ) and $<24 \mathrm{~h}$ after ED admission (10.0 [IQR 7.0-12.0] vs. 8.0 [IQR 6.0-11.0]; $\mathrm{P}<0.005$ ). At both time points, a median of $3 \mathrm{MDs}$ in chronic therapy was found (IQR 1.0-5.0 vs. IQR 2.0-5.0), mostly drug omissions (51.3\% vs. $55.9 \%)$. Inappropriate medications in chronic therapy $(30.8 \%$ vs. $36.3 \%)$ and untreated indications in prescribed medication (26.8\% vs. $33.2 \%)$ occurred as most frequent DRPs. Significant severe DRPs were common in chronic therapy (58.6\% vs. $59.6 \%)$ and prescribed medication $(72.7 \%$ vs. $69.1 \%)$. Two-thirds $(65.8 \%)$ of the PIs were fully accepted and implemented by the ward physician.

Conclusion: Presence of a 24/7 CP in the ED could have an added value in patient care and medication safety because of interception of MDs and DRPs after performing MR and reviewing prescribed medications both during nighttime hours as $<24 \mathrm{~h}$ after ED admission.

\section{PP032}

Results of the gamer trial: gaming for adherence to medication using e-health in rheumatoid arthritis

\author{
Bart Pouls ${ }^{*}$ 1, 2 , Charlotte Bekker ${ }^{1}$, Joke Vriezekolk ${ }^{2}$, Sandra van \\ Dulmen $^{3,4}$, Bart van den Bemt ${ }^{1,2}$ \\ ${ }^{1}$ Pharmacy, RadboudUMC, ${ }^{2}$ Research, Sint Maartenskliniek, \\ ${ }^{3}$ Primary and Community Care, RadboudUMC, ${ }^{4}$ Nivel (Netherlands \\ institute for health services research), Nijmegen, Netherlands
}

Background and Objective: Effectiveness of pharmacological therapy in rheumatoid arthritis (RA) is limited by inadequate medication adherence. Medication adherence can be influenced by implicit (unconscious) judgements concerns about adverse consequences. We targeted these implicit judgments using a serious puzzle game.

Aim To examine the effectiveness of a serious game to improve medication adherence in patients with rheumatoid arthritis treated with anti-rheumatic drugs.

Method: A multi-centre randomised clinical trial was performed with a 3 month follow-up period.*

Inclusion criteria were adulthood, rheumatoid arthritis, use of antirheumatic drugs and possession of a smartphone/tablet. Control patients received usual care whereas intervention patients were invited to install and play the serious puzzle game at will. 
Main outcome measures: Primary outcome was proportion of adherent patients at three months as scored by the Compliance Questionnaire in Rheumatology. Additionally, beliefs about medication, Rheumatoid Arthritis Disease Activity Index, app usage data and app usability were collected.

Results: In total 187 patients completed the study. Of the 104 intervention patients, 93 (89\%) played the intervention. Median playtime was 444 minutes (IQR 156 - 1269 minutes). Usability score was 65 out of 100 . Adherence and disease activity data is currently being analysed and will be presented during the conference.

Conclusion: A serious puzzle game that targets implicit medication judgments was frequently played during a randomised intervention study. We are looking forward to present the data on its effectiveness during the conference.

References: $*$ Netherlands Trial Register NL7217: https://www.trialregister.nl/trial/7217

\section{PP033}

Identifying determinants and consequences of drug-induced acute kidney injury (AKI)

\section{Catarina da Luz Oliveira* 1, 2, Filipa Duarte-Ramos ${ }^{3}$, Fernando Fernandez-Llimos ${ }^{4}$, Filipa Alves da Costa ${ }^{3}$}

${ }^{1}$ Hospital Pharmacist, Hospital Vila Franca de Xira, ${ }^{2} \mathrm{PhD}$ student, Faculty of Pharmacy, University of Lisbon, ${ }^{3}$ Assistant Professor, Faculty of Pharmacy, University of Lisbon, Lisboa, ${ }^{4}$ Assistant Professor with aggregation, Faculty of Pharmacy, University of Porto, Porto, Portugal

Background and Objective: Acute kidney injury (AKI) is a common syndrome in hospitalized patients and associated with short and longterm morbidity and mortality, often induced by exposure to AKIinducing drugs. A possible consequence of the use of nephrotoxic drugs is an increased risk of progression to chronic renal failure and adverse drug reactions (ADRs) are one of the most important modifiable factors in the context of AKI. The absence of specific treatment calls for the need for prompt clinical identification of creatinine changes.

To contribute to the development of a clinical pharmacist intervention program to minimize drug-induced AKI. The intermediate goals are: to evaluate the evolution of the glomerular filtration rate (GFR), as a marker of prognosis of kidney injury, in patients admitted to the Vila Franca de Xira Hospital (HVFX) and treated with drugs with nephrotoxic potential; and to estimate the prevalence of Acute Renal Insufficiency as ADRs reported to the Portuguese national pharmacovigilance database and describe drugs more frequently involved.

Design: To meet the first goal, a retrospective cohort study will follow patients admitted to the HVFX between January 1, 2019 and December 31, 2020 and treated with drugs with nephrotoxic potential and evaluate the evolution of the glomerular filtration rate (GFR), as a prognostic marker of kidney injury. The length of stay, sociodemographic and clinical characteristics of the patient will be explored as potential predictors of iatrogenic renal damage during hospital stay. The analysis will be performed using SPSS v.25 and RStudio v.1.2 software. The analysis will include univariate, bivariate and multivariate descriptive statistics. In the bivariate and multivariate analysis, a $95 \%$ confidence interval will be used. To achieve goal 2, a case-non case study will be established resorting to the Regulatory agency database for the period between 01/01/2009 and 12/31/2020. Cases will be defined based on the SMQ survey of Acute Renal Insufficiency that covers the MedDRA terms for this condition; all remaining reports are non-cases. Reporting Odds Ratios (ROR) will be used to describe the differential risk of drugs involved.
Results: No results are available yet; the aim of this presentation is to discuss the methodological approach taken.

Conclusion: The results of this work are expected to contribute to a deeper understanding of the AKI-inducing drugs and how other factors may change the odds of occurrence of AKI in real-world. This will thus lead to the development of clinical pharmacy evidencebased interventions to be implemented in future work.

\section{PP034}

Investigating the effect of a clinical medication review including pre-emptive pharmacogenotyping in patients with major depression—a study design

Céline K. Stäuble ${ }^{* 1, ~ 2, ~} 3$, Martin Hatzinger ${ }^{4}$, Kurt E. Hersberger ${ }^{2}$, Markus L. Lampert ${ }^{1,2}$, Henriette E. Meyer zu Schwabedissen ${ }^{3}$, Diana Spieker ${ }^{4}$, Thorsten Mikoteit ${ }^{4}$

${ }^{1}$ Institute of Hospital Pharmacy, Solothurner Spitäler, Olten,

${ }^{2}$ Pharmaceutical Care, Department of Pharmaceutical Sciences,

${ }^{3}$ Biopharmacy, Department of Pharmaceutical Sciences, University of Basel, Basel, ${ }^{4}$ Psychiatric Services, Solothurner Spitäler and

Department of Medicine, University of Basel, Solothurn, Switzerland

Background and Objective: Finding the right drug and dosage for the patient remains challenging in many cases and inter-individual differences in response are frequently observed. Especially, for antidepressants, it is known that about $50 \%$ of the patients do not respond to the prescribed first-line medication after four weeks of treatment, resulting in an immense burden for the patient. One reason for inter-individual differences in drug response is based on genetic predisposition. Today, a number of guidelines for pharmacogenetic (PGx) guided drug selection and dosing are available. This is also the case for antidepressant compounds namely for selective serotonin reuptake inhibitors and tricyclic antidepressants. Still, there is not yet enough data from prospective studies to favour PGx-guided treatment over standard care in psychiatric practice. We hypothesize that alongside with accepted factors like drug-drug interactions and disease modalities, there is enough evidence to beneficially incorporate PGx information to guide drug selection and dosing in the treatment of depression.

Design: With the aim to investigate the effect of pre-emptive pharmacogenotyping in combination with a clinical medication review in psychiatric practice, we designed a prospective, randomized, open label clinical trial. The study intervention is a consultation of the psychiatrist with a clinical pharmacist in order to support clinical decision making for antidepressant selection and dosing. This consultation includes genotyping and thereof evidence-based genotype interpretation in addition to a clinical medication review considering the individual patient history with previous therapy failures or ADEs, medical and laboratory data (incl. serum drug levels where available) as well as current co-medication. The included patients are randomized to either being treated without prior PGx assessment and without a pharmacist consultation (standard care), or to receive a drug treatment based on a pharmacist consultation including PGx information obtained from a pharmacogenetic panel testing.

Results: The primary study outcome is treatment response based on the Hamilton Depression Rating Scale (HAM-D) after four weeks of treatment. Further secondary outcomes include time to response, remission rate, overall change in HAM-D, duration of hospitalization, number of adverse events related to the antidepressant pharmacotherapy and patient self-evaluation of depression and side effects. Conclusion: The herein described study design was developed and implemented in close collaboration of clinical pharmacists with psychiatrists. We therefore expect valuable and relevant insight into 
pre-emptive pharmacogenotyping as a clinical pharmacy service in psychiatry practice and its potential benefits for major depression patients.

\section{PP035}

Clinical pharmacist-led medication reconciliation service in patients with infectious disease during COVID-19 pandemic

\section{Cüneyd Enver $^{*}{ }^{1}$, Buket Ertürk Şengel ${ }^{2}$, Mesut Sancar ${ }^{1}$, Volkan Korten $^{2}$, Betul Okuyan ${ }^{1}$}

${ }^{1}$ Department of Clinical Pharmacy, Marmara University Faculty of Pharmacy, ${ }^{2}$ Department of Infectious Disease and Clinical Microbiology, Marmara University School of Medicine, Istanbul, Turkey

Background and Objective: Clinical pharmacist-led medication reconciliation service could be decreased medication discrepancies which are resulted in medication errors and adverse drug events during transitions of care. The aim of the study is to develop the clinical pharmacist-led medication reconciliation service in patients with infectious disease during COVID-19 pandemic.

Method: In this prospective observational study, the clinical pharmacist-led medication reconciliation service (both at admission and discharge) has been provided in patients ( $>18$ years old) with infectious disease (including COVID-19). This study was conducted at the infectious diseases and internal medicine inpatient services of an education and research hospital between July 2020 and February 2021. Besides, the clinical pharmacist-led the patient education and counselling (by using teach-back technique, providing patient pill card including pictograms, and brochures) was provided the patients face to face and/or telephone (especially for patients with COVID-19) at discharge.

Main outcome measures: The number of medications, Charlson Comorbidity Index, the LACE Index, SCOREM (Score to Assess Risk of Medication Errors), medication discrepancy based on Med$\operatorname{Tax}^{1}$, thirty days hospital readmission and admission to emergency department after discharge, patient reported medication adherence.

Results: Among 154 patients (female/male: 83/71), the mean age of them was $61.3 \pm 15.1$. The median values for the number of medications at admission and discharge were 5.0 (IQR: 3.0-8.0) and 6.0 (IQR: 5.0-9.0); respectively. The median of length of stay was 10.0 (IQR: 6.0-15.0) days. The median score of Charlson Comorbidity Index was 3.0 (IQR: 2.0-4.2). The median score of the LACE Index was 12.0 (IQR: 10.0-14.0). The patients with COVID-19 had significantly less score of the LACE Index when compared with patients without COVID-19 $(p<0.05)$. The median score of SCOREM was 2.0 (IQR: 2.0-3.0). The numbers of medication discrepancies at admission and discharge were 1211 (intentional/unintentional=998/213) and 329 (intentional/unintentional=326/3); respectively. The most common recommendations of the clinical pharmacist were related to the addition of omitted medication. Of them, $12.3 \%$ had 30 days hospital admission and $15.8 \%$ admitted to emergency department. During a month follow-up call after discharge, twenty-three patients $(17.6 \%)$ stated they skipped at least one dose of their medications at last week.

Conclusion: Clinical pharmacist-led medication reconciliation service (including patient education and counselling [with telepharmacy support]) could be decreased the number of medication discrepancies both at admission and discharge in patient with infectious disease.

References: 1. Almanasreh E, Moles R, Chen TF. The medication discrepancy taxonomy (MedTax): The development and validation of a classification system for medication discrepancies identified through medication reconciliation. Res Social Adm Pharm. 2020

\section{PP036}

Ursodeoxycholic acid role in drug-induced liver injury: a critically ill patient case report

\section{Dina Mendes" ${ }^{*}$, Ana Sousa ${ }^{2}$, Helena Farinha ${ }^{1,3}$, Eduarda Carmo $^{2}$, Fátima Falcão ${ }^{3,4}$}

${ }^{1}$ Pharmacy, ${ }^{2}$ Polyvalent Intensive Care Unit, Hospital Egas Moniz, Centro Hospitalar Lisboa Ocidental, ${ }^{3}$ Faculty of Pharmacy, University of Lisbon, Lisbon, ${ }^{4}$ Pharmacy, Centro Hospitalar Lisboa Ocidental, Lisboa, Portugal

Background and Objective: Drug-induced hepatotoxicity caused by beta-lactam antibiotics or antifungals is well recognized and can result in acute liver failure with significant morbidity to patients, even death. Early recognition of this event and withdrawal of the causative drugs is critical to prevent further deterioration in liver function. Today, ursodeoxycholic acid (UDCA) is referred only as a possible option in clinical guidelines for treatment of drug induced liver injury (DILI) because of limited evidence regarding efficacy, given the absence of controlled and well documented studies. However, there has been some reports of benefit in DILI.

Design: We describe the case of a 66-year old male patient admitted in Intensive Care Unit (ICU) with respiratory insufficiency due to SARS-CoV-2 pneumonia, history of renal transplantation and chronic B hepatitis. At day 8 of admission, he presented an altered liver function (peak alanine aminotransferase (ALT) value of $193 \mathrm{U} / \mathrm{L}$, peak aspartate aminotransferase (AST) value of $146 \mathrm{U} / \mathrm{L}$, peak gamma-glutamyl transferase (GGT) value of $255 \mathrm{U} / \mathrm{L}$, peak lactate dehydrogenase level of $685 \mathrm{U} / \mathrm{L}$, peak total bilirubin $6.15 \mathrm{mg} / \mathrm{dL}$ ), jaundice and acholia. After excluding differential diagnosis, including flare of subjacent hepatic disease, we performed a literature review and assumed a mixed DILI, hepatocellular and cholestatic, probably due to piperacillin/tazobactam, voriconazol and isavuconazol. These drugs were stopped but liver function continued declining. Two days after, we started treatment with UDCA $250 \mathrm{mg}$ three times a day by nasogastric tube.

Results: After initiating UDCA, liver function tests began to improve and in 4 days the patient became less icteric and acholia resolved. In a week, ALT was $74 \mathrm{U} / \mathrm{L}$, AST $53 \mathrm{U} / \mathrm{L}, \mathrm{LDH} 368 \mathrm{U} / \mathrm{L}$ and total bilirrubine $1.53 \mathrm{mg} / \mathrm{dL}$. There was no side effects.

Conclusion: Administration of UDCA to critically ill patients presenting DILI may attenuate hepatotoxicity, preventing serious liver disease and avoiding the restriction in utilization of other drugs with possible hepatic adverse effects. Further studies are needed to document the true potential of UDCA in DILI with cholestatic features. ICU pharmacists have a central role in critically ill patient drug management and optimal pharmaceutical care within the health team.

\section{PP037}

Exploring pharmacogenomics: re-using available mendeliome data and determining patient perspectives

\section{Eline Coene* $^{1}$, Catharina Olsen ${ }^{2}$, Mathijs Swaak ${ }^{1}$, Freya Vaeyens $^{2}$, Frederik Hes ${ }^{2}$, Stephane Steurbaut ${ }^{1}$, Sonia Van Dooren $^{2}$, Pieter-Jan Cortoos ${ }^{1}$}

${ }^{1}$ Pharmacy, ${ }^{2}$ Centrum for Medical Genetics, UZ Brussel, Brussels, Belgium

Background and Objective: The Centre for Medical Genetics (CMG) of UZ Brussel, a 729-bed tertiary hospital in Brussels (Belgium), has been performing next generation sequencing of mendeliomes for diagnostic purposes since 2016. Pharmacogenomic 
data is thus already available, but currently not used. We took the opportunity to explore the possibility of reporting pharmacogenomic information as 'secondary findings' and to investigate the interest in and required features of a future pharmacogenomic project.

Method: We retrospectively reviewed available genomic data for patients that had a mendeliome analysis between 01/03/2016 and $30 / 06 / 2020$. Fourteen pharmacogenes comprising 626 loci were selected for review. To enable haplotype assignment, we developed a basic tool that displayed all possible haplotypes with a corresponding 'matching score' and 'completeness score'. Where possible, phasing was done based on pedigree information. The resulting phenotypes were further compared with medication histories, abstracted from the electronic medical record. Additionally, a 24-question survey was conducted between 21/12/2020 and 09/05/2021 among patients at the CMG of UZ Brussel exploring general interests and attitudes and beliefs about sharing and using pharmacogenomic tests.

Main outcome measures: We aimed to explore (1) the prevalence of actionable pharmacogenes and gene drug interactions (GDIs) and (2) attitudes regarding pharmacogenomic testing amongst CMG patients. Results: Pharmacogenomic data could be used for 536 individual patients, revealing that at least $76.9 \%$ had one or more actionable phenotype and 60 GDIs with varying relevance were found. CYP2C9 had the most actionable phenotypes (174/536) and was involved with 42 GDIs (e.g. 4 GDIs with phenytoin). However, not all phenotypes were detectable for CYP2C19 and CYP2D6. The survey was completed by 60 individuals showing considerable interest in pharmacogenetic tests, partially dependent on reimbursement $(56 / 60$ when fully reimbursed). Respondents expressed interest in obtaining additional information about their results through a website (47/57) or healthcare provider (52/60), and would feel comfortable sharing their data through an electronic platform $(48 / 60)$ or a pharmacogenomic passport $(53 / 60)$ with all or a selection of healthcare providers (54/ $60)$, mainly physicians (58/60). Nevertheless, concerns remained regarding privacy (35/60) and possible misuse of this data (25/60).

Conclusion: Reusing genomic data has great potential. This project may provide an impetus for a future pharmacogenomic initiative at UZ Brussel and other hospitals. More research and debate among patients, healthcare providers and other actors will be necessary to meet best practices.

\section{PP039}

Does interruptive medication alerting in CDSS increase alert acceptance?

Janina A. Bittmann ${ }^{* 1,2}$, Michael Metzner ${ }^{2}$, Walter E. Haefeli ${ }^{1,2}$, Hanna M. Seidling ${ }^{1,} 2$

${ }^{1}$ Cooperation Unit Clinical Pharmacy, ${ }^{2}$ Department of Clinical Pharmacology and Pharmacoepidemiology, Heidelberg University Hospital, Heidelberg, Germany

Background and Objective: Clinical Decision Support Systems (CDSS) generate medication alerts during inpatient care when critical constellations of patient and medication parameters are identified. Override rates may reach almost $100 \%$ [1-3], however, might be mitigated by considering patient characteristics [4], alert tailoring [1], display of severe alerts, interruptivity, or a combination of all $[5,6]$. Depending on the alert type, acceptance rates in literature could be improved up to $67 \%[4,5]$. Therefore, we prospectively collected and retrospectively analyzed the acceptance rate of interruptive alerts in prescription constellations with a particularly high risk of adverse drug events.

Method: Medication and alert data collected at Heidelberg University Hospital between 03/2016 and 08/2020 were considered. In total, 31 distinct alerts for contraindicated drug-drug interactions (DDI) with simvastatin, potentially inappropriate medication for patients over 65 years (PIM, $\mathrm{N}=14$ drugs and 36 drug combinations), or contraindicated drugs in the presence of hyperkalemia $(\mathrm{N}=5)$ could be accepted or overridden giving a reason in free-text form, which was also taken into account when assessing the acceptance. Differences in parameters influencing alert acceptance were calculated using the chisquare test considering a two-tailed $\mathrm{p}$-value of $<0.05$ as significant. Main outcome measures: Acceptance rate of interruptive medication alerts measured as change in medication orders.

Results: In total, $57.5 \%$ (269/468 alerts) of the evaluable alerts were accepted while $42.5 \%$ were overridden continuing the prescription despite interruptive alerting. Acceptance rates differed significantly according to the alert type $(\mathrm{p}<0.0001)$. Most $(85.7 \%)$ DDI alerts were accepted (mostly simvastatin-clarithromycin) by discontinuation of at least one interacting drug. Contraindications in the presence of hyperkalemia (mostly caused by spironolactone) were accepted in $65.3 \%$ whereas the acceptance rate of PIM alerts was $25.1 \%$ (mostly immediate-released nifedipine).

Conclusion: More than half of the interruptive drug alerts were accepted in our setting, with DDI alerts being the most commonly accepted. However, only about one in four PIM alerts was accepted even though they were interruptive. In contrast to the DDI warnings and contraindication alerts for hyperkalemia, PIM alerts only warned of high-risk situations, which were not a contraindication, possibly explaining why the alert was less often considered by the user.

References:1. van der Sijs H, Aarts J, Vulto A, et al., Overriding of drug safety alerts in computerized physician order entry, J Am Med Inform Assoc, 2006;13(2):138-47.

2. Seidling HM, Klein U, Schaier M, et al., What, if all alerts were specific - estimating the potential impact on drug interaction alert burden, Int J Med Inform, 2014;83(4):285-91.

3. Ancker JS, Edwards A, Nosal S, et al., Effects of workload, work complexity, and repeated alerts on alert fatigue in a clinical decision support system, BMC Med Inform Decis Mak, 2017;17(1):36.

4. Muylle KM, Gentens K, Dupont AG, et al., Evaluation of context-specific alerts for potassium-increasing drug-drug interactions: A pre-post study, Int J Med Inform, 2019;133:104013.

5. Cornu P, Steurbaut S, Gentens K, et al., Pilot evaluation of an optimized context-specific drug-drug interaction alerting system: A controlled pre-post study, Int J Med Inform, 2015;84(9):617-29.

6. Ramirez M, Maranon R, Fu J, et al., Primary care provider adherence to an alert for intensification of diabetes blood pressure medications before and after the addition of a "chart closure" hard stop, J Am Med Inform Assoc, 2018;25(9):1167-1174.

\section{PP040}

Effectiveness and safety of alglucosidase alfa, by the purpose of a case

Javier Garcia Marin ${ }^{*} 1$, Javier Romero Puerto ${ }^{1}$, Sol Cortés de Miguel $^{1}$, Alberto Soria Martin ${ }^{1}$, Adriana Moreno Herrera ${ }^{1}$

\section{${ }^{1}$ HOSPITAL PUNTA DE EUROPA, ALGECIRAS, Spain}

Background and Objective: Pompe disease or type II glycogenosis is a rare hereditary disease of an autosomal recessive nature. It is caused by a deficiency of the enzyme alpha 1-4 glucosyl transferase. This deficit produces an accumulation of glycogen in tissues, causing respiratory, cardiac and motor failure. The objective is to analyze the efficacy and safety of treatment with alglucosidase alfa in a patient diagnosed with late-onset Pompe disease. 
Design: The data were obtained by analyzing the patient's clinical history in Jara ${ }^{\circledR}$. The efficacy variables used to assess the evolution were: motor function (using timed tests: 6-minute test, going up and down the steps and time to walk 10 meters), muscle enzymes (creatine phosphokinase), subjective perception of quality of life (WHOQOL questionnaire), forced vital capacity and anti-alglucosidase alfa antibodies. The study covers from October 2019, the date on which treatment began, until April 2020. The dose of alglucosidase alfa used was $20 \mathrm{mg} / \mathrm{kg}$ intravenous weight every 2 weeks. At the date of the study, the patient had received a total of 41 administrations.

Results: During the study period, the patient worsened his subjective feeling of quality of life due to greater intensity of myalgia, fatigue and the onset of myopathic gait. The timed tests were kept constant from the start of treatment. Creatine phosphokinase values decreased from $479 \mathrm{IU} / \mathrm{L}$ at the start of treatment to $214 \mathrm{IU} / \mathrm{L}$ at 12 months (absolute reduction of $255 \mathrm{UI} / \mathrm{L}$ ). The anti-alglucosidase antibody titers were $1 / 1600$ at 3 months after onset and $1 / 800$ at 12 months. Regarding safety, during the study period there were no adverse reactions attributable to the drug, except for syncope produced in the first infusion, which could not be related to the drug. Spirometries performed at the beginning and at month 12 showed stability of the disease in terms of forced vital capacity.

Conclusion: During the study period, there has been a decrease in creatine phosphokinase concentrations and the timed test values have remained constant. However, the patient's sense of quality of life has diminished. Anti-alglucosidase alfa antibody titers, related to infusion reactions, are decreasing. Therefore, we consider that in our case the drug has not shown a relevant benefit in relation to its high economic impact. On the other hand, alglucosidase alfa has been shown to be a safe drug.

\section{PP041}

Detection of interactions with capecitabin as part of the hospital pharmaceutical care

Marina Fages Perez ${ }^{1}$, Adriana Moreno Herrera ${ }^{1}$, Javier Romero Puerto $^{2}$, Javier Garcia Marin* 1, Alberto Soria Martin ${ }^{1}$

${ }^{1}$ FARMACIA, HOSPITAL PUNTA DE EUROPA, ALGECIRAS, ${ }^{2}$ HOSPITAL NAVALMORAL DE LA MATA, CACERES, Spain

Background and Objective: The objective of this research is the detection of the most important pharmacological interactions that occur between the patient's home medication and capecitabine, to know the amount in which they are given, to report the interactions detected in case patients maintained active interaction during the research, and to quantify the acceptance by the clinicians of the pharmaceutical intervention.

Design: Retrospective observational study, in a specialty hospital, from June 2019 to February 2020, of cancer patients who received capecitabine. Data were obtained from the PRISMA-APD outpatient care program, and by reviewing home medication in the Digital Unique History (Diraya). The interactions reviewed for their high importance were capecitabine with allopurinol, acenocoumarol, warfarin, phenytoin, or proton pump inhibitors (PPIs). Clinician acceptance was assessed with the number of treatment modifications after our recommendation. Other variables collected were: age, sex and type of cancer.

Results: 61 patients were included in the study, 36 men and 25 women, with a mean age of 66.5 years, all of them on capecitabine treatment. 54\% had colon cancer, $23 \%$ rectal cancer, $13 \%$ breast cancer and $10 \%$ other digestive cancers. Interactions were detected in 32 of the analyzed patients, all of them due to the association of
Capecitabine with PPI and without detecting any other interaction considered relevant. Of these 32 patients, 25 kept active the treatment with capecitabine during the research cutoff (February 2020), maintaining a PPI as part of their home medication in 15 of them, and prescribers were informed of this interaction. In 8 of the 15 patients, the clinicians accepted the recommendation, and their treatment with PPI was temporarily suspended, or it was changed to another antacid without interaction with Capecitabine.

Conclusion: In a large number of cancer patients who are receiving capecitabine, interaction occurs between capecitabine and a PPI, prescribed as home medication. The role of the pharmacist is key to detecting this type of interactions, since half of the prescriptions in this research were modified by clinicians when they were informed of this interaction. Pharmaceutical intervention helps that the efficacy of Capecitabine is not affected by a decrease in the bioavailability of the drug at its target.

\section{PP042}

Pharmaceutical blended-care for cancer outpatients: needs and expectations

Joana R. Ribeiro ${ }^{*}{ }^{1,}$, Ilyse Kenis ${ }^{3}$, Veerle Foulon ${ }^{3}$, Hélder D. Mota-Filipe $^{2}$, Filipa A. da Costa ${ }^{2}$

${ }^{1}$ Hospital Pharmacy, Portuguese Institute of Oncology, ${ }^{2}$ Research Institute for Medicines (iMed.ULisboa), Faculty of Pharmacy, University of Lisbon, Lisbon, Portugal, ${ }^{3}$ Department of

Pharmaceutical and Pharmacological Sciences, KU Leuven, Leuven, Belgium

Background and Objective: The COVID-19 pandemic situation has been adding further challenges to the functioning of the National Health System. To improve access to health care, telehealth was encouraged and hospital medicines proximity access measures were adopted. Pharmaceutical Care (PC) is defined as the responsible provision of drug therapy. Thus, there is a perceived need to study how PC could be improved to effectively monitor, remotely, oral therapy for cancer. The purpose of this study is to assess needs and expectations of cancer outpatients and healthcare professionals about the provision of PC.

Design: A qualitative study will be conducted to evaluate: patients' needs for support in medication use, views of healthcare professionals on interdisciplinary collaboration, and pharmacists' expectations regarding supportive tools. Grounded Theory method will be applied to sampling, data collection and analysis. Participants will be purposively selected to gain insight in the challenges and opportunities for improvement in oncology PC in Portugal. Participants will be allocated in focus groups according to their position in the patient care pathway: (1) cancer outpatients; (2) doctors and nurses; (3) pharmacists. Two focus groups for each position will be conducted. Data obtained will be coded and inductively analysed using thematic analysis.

Results: This study has been set up for the purpose of designing a patient-centred Pharmaceutical Blended-Care model that could also take into account the needs of healthcare professionals intervening in the care of cancer patients. The results of this study will allow the creation of that model.

Conclusion: In the new innovative PC model oncology PC will be adapted to the current reality, as well as to the expectations and needs of cancer patients and healthcare professionals. Facing the adversities of confinement caused by the COVID-19 pandemic, it is expected that this model improves PC in oncology. The creation of a theoretical model, based on face-to-face and remote PC, is part of a larger study which also intends to test this model. 


\section{PP043}

Direct oral anticoagulants (DOACs) use in patients with cardiac implantable devices and non-valvular atrial fibrillation: impact of off-label dose use in safety and efficacy outcomes

\section{Joana Santos ${ }^{*}$, Natália António ${ }^{2,3,4}$, Lino Gonçalves 2, 3, 4 , Marília J. Rocha ${ }^{5}$, Ana Fortuna ${ }^{1,6}$}

${ }^{1}$ Laboratory of Pharmacology, Faculty of Pharmacy, University of Coimbra, ${ }^{2}$ Coimbra Institute for Clinical and Biomedical Research (iCBR), ${ }^{3}$ Cardiology Department, Coimbra Hospital and Universitary Centre, ${ }^{4}$ Faculty of Medicine, University of Coimbra,

${ }^{5}$ Pharmaceutical Service, Coimbra Hospital and Universitary Centre, ${ }^{6}$ CIBIT/ICNAS - Coimbra Institute for Biomedical Imaging and Translational Research, University of Coimbra, Coimbra, Portugal

Background and Objective: Direct oral anticoagulants (DOACs) are the first line therapy for stroke prevention in non-valvular Atrial Fibrillation (NVAF). However, observational studies are evidencing more negative outcomes than expected in the clinical trials [1]. Moreover, the use of off-label doses is related to an increased risk of stroke, bleeding and/or adverse effects [2,3]. This real-world population study aimed to evaluate the DOACs use in safety and efficacy outcomes in patients with cardiac implantable electronic devices (CIEDs).

Method: A single-centre observational retrospective study was performed enrolling all patients implanted with a CIED between January 2011 and January 2015 in Cardiology Department of Coimbra Hospital and Universitary Centre (Portugal). Therefore, 468 patients with NVAF and taking DOACs during the study time were included. This study was approved by FMUC Ethics Committee.

Main outcome measures: Baseline demographic and clinical characteristics were evaluated, and the dose adjustments were assessed according with European Medicines Agency (EMA) guidelines. All adverse bleeding and thromboembolic events reported during followup were considered as endpoints.

Results: In this cohort, mean age was $82.4 \pm 7.7$ years and $58.1 \%$ of the patients were male. During the follow-up period, 105 patients experienced a total of 139 adverse events. In this group, the mean time between DOAC initiation and the occurrence of any adverse bleeding or thromboembolic event was $21.2 \pm 20.7$ months. The highest percentage of the events observed were hemorrhagic $(57.6 \%)$, but most of them were considered minor bleedings. More than a half of the events were observed with dabigatran $(53.2 \%)$, followed by rivaroxaban an apixaban, with the same percentage of events registered $(23.0 \%)$. Regarding doses, $70.5 \%$ of the overall population were taking reduced doses and $7.2 \%$ were taking non-approved DOAC doses for NVAF. Concerning dose adequacy, it was demonstrated that $29.5 \%$ of events were reported in patients administered with off-label doses, according to EMA guidelines: $21.6 \%$ of them received underdosed off-label doses while $7.9 \%$ were overdosed.

Conclusion: A significant proportion of the adverse events identified in this population of NVAF patients with CIEDs under DOAC therapy occurred in patients taking off-label DOAC doses, highlighting the real-world problematic of underdosing. The use of off-label doses can jeopardize the efficacy and safety of DOACs, in real-world practice, and all clinical data must be considered to improve DOACs dose adjustments.

References: [1] Noseworthy PA et al. Long-term stroke and bleeding risk in patients with atrial fibrillation treated with oral anticoagulants in contemporary practice: Providing evidence for shared decisionmaking. Int J Cardiol. 2017; 245:174-177. https://doi.org/10.1016/ j.ijcard.2017.07.043

[2] Cho MS et al. Pattern and Impact of Off-label Underdosing of Non-Vitamin K Antagonist Oral Anticoagulants in Patients With
Atrial Fibrillation Who are Indicated for Standard Dosing. Am J Cardiol 2020;125:1332-1338. doi: 10.1016/j.amjcard.2020.01.044

[3] Santos J et al. Impact of direct oral anticoagulant off-label doses on clinical outcomes of atrial fibrillation patients: A systematic review. Br J Clin Pharmacol. 2020;86(3):533-547. doi: 10.1111/ bcp.14127.

\section{PP044}

Opioid withdrawal guidelines may help to improve the initiation of opioid substitution therapy (OST) in acute hospitals

\section{Kevin Cahill' ${ }^{1}$, Sana $\mathrm{Ali}^{1}$, Gideon Mlawa ${ }^{1}$, Hassan Rehamni ${ }^{1}$,} Musarat Hussain ${ }^{1}$

${ }^{1}$ Barking Havering and Redbridge University Trust, Romford, United Kingdom

Background and Objective: In 2018 there were 7,545 drug related hospital admissions in England \& Wales. 1 in 12 (8.5\%) of adults aged 16-59 years have used illicit drugs in the last 12 months. Patients who misuse drugs, substances and alcohol may attend emergency departments or be admitted to acute hospitals for conditions either directly related to or coincidental to their substance abuse. Although there are nationally established guidelines for the management of alcohol withdrawal, the same cannot be applied to opioid dependence. Local Trust guidelines developed and implemented in 2019 to manage opioid dependent adult patients. This audit was completed to evaluate adherence to the prescribing guidelines for opioid substitution therapy (OST) to local guidelines for opioid dependent adult patients at Barking, Havering and Redbridge University Hospitals Trust (BHRUT)

Design:

- Data collected from November 2019 to October 2020 at one hospital site.

- 10 patients identified retrospectively using pharmacy issue data.

- Clinical notes requested and audited by junior doctor against local standards.

- Results assessed against standards.

- Pregnant patients and those under 18 excluded.

Standards

1. $100 \%$ of all methadone issues to inpatient areas should be allocated to a named patient.

2. $100 \%$ of patients who are initiated on methadone after admission to BHRUT should be monitored using a Clinical Opioid Withdrawal Score (CWS).

3. $100 \%$ of patients who are established on methadone as OST and are admitted to hospital should have their usage confirmed by pharmacy lead medicines reconciliation before an inpatient prescription is initiated.

4. $100 \%$ of methadone OST prescriptions should be prescribed as per the Trust's protocol.

\section{Results:}

- Standard 1-0\% (0/172) standard NOT met

- Standard 2-100\% (2/2) standard met

- Standard 3-38\% (3/8) standard NOT met

- Standard 4-30\% (3/10) standard NOT met

Conclusion: There are robust guidelines nationally which are widely followed for the management of patients admitted directly because of or coincidentally with alcohol dependence, yet there are none for those with opioid dependence. Managing these patients well and 
ensuring they have the correct medication means they are less likely to abscond, and all round have better care. BHRUT developed its own guidelines in November 2019, with a very clear algorithm to follow on how to manage these patients. However, on the whole, this audit shows that these guidelines are not being implemented consistently. Recomendations

1. OST should be issued to inpatients on a named patient basis

2. OST guidelines should be included as part of clinical induction for all prescribers.

3. Pharmacy lead medicines reconciliation must be completed before the continuation of community OST as an inpatient.

4. Feedback the need for pan-regional or national guidelines for opioid dependent patients who are admitted to hospital.

\section{PP045}

Drug-related problems in a medicinal intensive care unit detected by clinical pharmacists on bedside rounding teams

Khanh H. P. Nguyen* ${ }^{1}$, Lan H. Vo ${ }^{2}$, Dung T. Nguyen ${ }^{2}$, Hai N. Truong ${ }^{3}$, Hoai T. T. Vu ${ }^{3}$, Thang T. Khong ${ }^{3}$

${ }^{1}$ Pharmacy, Vinmec Central Park hospital, ${ }^{2}$ Department of Clinical Pharmacy, School of Pharmacy, University of Medicine and Pharmacy at Ho Chi Minh City, ${ }^{3}$ Intensive Care Unit, Vinmec Central Park hospital, Ho Chi Minh, Viet Nam

Background and Objective: Intensive care unit (ICU) patients often require complex drug regimens, which may increase the risk of drugrelated problems (DRPs). Based on studies carried out worldwide, implementing clinical pharmacy services in critical care teams can reduce DRPs and improve healthcare quality. However, in Vietnam, the team-based care model in ICU with clinical pharmacists' participation has not yet been well-established. Additionally, there is no published interventional study on DRPs in Vietnam. Thus, the aims of this study were to identify DRPs in ICU patients and the clinical impact of interventions made by pharmacists during patient rounds. Method: A descriptive study from May 2018 to July 2020 was performed in a medical ICU in Ho Chi Minh City, Vietnam.Since January 2019, a clinical pharmacist was integrated in the interprofessional daily rounding team to detect and prevent DRPs. Detected DRPs during the study time were categorized by a modified PCNE classification system. A comparison of DRPs before and after clinical pharmacy inclusion in ICU rounds was done.

\section{Main outcome measures: Type of DRPs in ICU patients}

Clinical impact of interventions made by pharmacists during patient rounds.

Results: 56 and 152 patients were included before and after pharmacist's participation, respectively. 36\% (20/56) and 86\% (131/152) of them experienced 43 and 523 DRPs, respectively. The rate of detected problems per patient increased from 0.8 to 2.9 then to 4.7 in 3 consecutive years. Identified DRPs are often associated with anti-infective use but its proportion dwindled over time (from $65 \%$ in 2018 to $34 \%$ in 2020). Furthermore, the intervention rate before drug administration was around $64-72 \%$. Among interventions after drug administration, optimizing anti-infective dosage accounted for the highest percentage $(29 \%)$. The most frequent interventions were dosage adjustment (28\%) and instituting a new drug (25\%). Over $96 \%$ of the detected problems were resolved. Finally, the healthcare team also detected or prevented 136 adverse drug reactions and 19 drug interactions.

Conclusion: On-ward participation of a specialized clinical pharmacist in ICU healthcare team enhanced therapy optimizationand medication safety thanks to early DRPs detection and active surveillance.

\section{PP046}

A national survey of hospital pharmacists' views on pharmacist prescribing in the Irish hospital setting

\author{
Clíona Collins ${ }^{1}$, Kieran Dalton* 1 \\ ${ }^{1}$ Pharmaceutical Care Research Group, School of Pharmacy, \\ University College Cork, Cork, Ireland
}

Background and Objective: Pharmacist prescribers are established in hospitals in many countries worldwide, and have been shown to be as efficacious and less error-prone compared to other prescribers. In countries like Ireland where pharmacist prescribing is not commonplace, there is a clear need to assess stakeholders' views to inform its potential development. Therefore, the primary objective of this study was to capture the views of hospital pharmacists' in Ireland on pharmacist prescribing in the hospital setting.

Method: A cross-sectional online survey was distributed via email in October 2020 to all Pharmaceutical Society of Ireland pharmacist registrants with 'hospital' as their practice area $(n=689)$. Closedended questions were analysed using Microsoft ${ }^{\circledR}$ Excel and IBM ${ }^{\circledR}$ SPSS Statistics 26. Open-ended questions underwent thematic analysis, facilitated by NVivo ${ }^{\circledR} 12$. Ethics approval was obtained prior to study commencement.

Main outcome measures: Views on pharmacist prescribing authority, its implementation, and the scope of practice.

Results: In total, 284 eligible responses were received. Most had no prescribing experience $(82.2 \%) ; 5.7 \%$ were actively prescribing, and $12.1 \%$ had prescribed previously but not at the time of the survey. Of pharmacists who had never prescribed, $71.5 \%$ felt competent to undertake some prescribing activities. Most non-prescribing pharmacists would like a prescribing role and felt underutilised by not having prescribing authority $(83.6 \%)$, with almost half feeling frustrated $(45.7 \%)$ and undervalued $(45.5 \%)$ without prescribing. At least $86 \%$ of respondents believed that pharmacist prescribing allowed for increased workflow efficiency, faster error correction, along with increased professional autonomy and job satisfaction. Furthermore, at least $88 \%$ perceived that its routine introduction in Irish hospitals would reduce errors, increase patient safety and prescribing appropriateness, reduce other prescribers' workload, and improve transitions of care. Nearly all respondents wanted specific legislation to give pharmacists prescribing authority $(91.9 \%)$. This absence of legislation, along with the lack of a prescribing training course in Ireland and resource issues (such as staffing) were the greatest perceived barriers to implementation.

Conclusion: This survey has shown widespread eagerness amongst pharmacists regarding the expansion of pharmacist prescribing in Irish hospitals, with expected benefits to the profession and patient care. Its success in other regions emphasises the need for prompt stakeholder engagement to implement specific legislation and training for pharmacist prescribing in Ireland. 


\section{PP047}

Drug-related problems experienced by patients using antirheumatic drugs: a longitudinal observational study

\section{Lex Haegens ${ }^{*}{ }^{1}$, Victor Huiskes ${ }^{2}$, Lisa-Marie Smale ${ }^{3}$, Charlotte Bekker $^{3}$, Bart van den Bemt $^{2,3}$}

${ }^{1}$ Department of Rheumatology Research, ${ }^{2}$ Department of Pharmacy, Sint Maartenskliniek, ${ }^{3}$ Department of Pharmacy, Radboud University Medical Center, Nijmegen, Netherlands

Background and Objective: Patients with rheumatic diseases using disease-modifying antirheumatic drugs (DMARDs) often experience drug-related problems (DRPs). Although DRPs can lead to a decreased quality of life and preventable hospital admissions, not much is known about the number and types of DRPs this population experiences over time. Therefore this study aims to longitudinally identify drug-related problems experienced by patients with rheumatic diseases using a disease-modifying antirheumatic drug.

Method: A prospective observational study was conducted in the outpatient pharmacy of the Sint Maartenskliniek between December 2019 and April 2020. Adult patients with a rheumatic disease using a DMARD were questioned about experienced DRPs by phone four times with two-week intervals using a structured interview-guide. DRPs were categorized using a seven-class patient-oriented classification system composed for this study, scored on uniqueness within patients, and analyzed descriptively.

Main outcome measures: Number and types of unique DRPs raised per participant.

Results: In total, 52 participants (age range $27-82$ years, $52 \%$ male) completed 192 interviews with $45(87 \%)$ participants completing all four interviews. $65 \%$ of participants were diagnosed with rheumatoid arthritis. Median (interquartile-range) number of unique DRPs raised per interview were 4 (2-6.75), $1(0.25-2), 1(0-3)$ and $0.5(0-2)$, for interview 1 to 4 respectively. In total, 426 unique DRPs were raised. 16 participants $(31 \%)$ reported at least one unique DRP in every interview completed, two participants $(4 \%)$ reported no DRPs. Reported unique DRPs related to drug side effects (24\%), good drug management $(23 \%)$, drug concerns $(22 \%)$, drug effectiveness $(20 \%)$, information needs $(8 \%)$, contra-indications $(2 \%)$ and logistics $(2 \%)$. Conclusion: Patients with rheumatic diseases using a DMARD experience multiple DRPs over time when actively questioned. This longitudinal observation of unique DRPs indicates that this population can benefit from continuous support regarding medication use.

\section{PP048}

Early detection from acute prerenal kidney damage in hospitalized patients through multidisciplinary action

Margarita Beltrán-García* 1, Alicia Aguado-Paredes 1 , Laura Moñino-Dominguez ${ }^{1}$, Santiago Sandoval-Fernández del Castillo ${ }^{1}$, Mercedes Salgueira-Lazo ${ }^{2}$, Antonio León-Justel ${ }^{3}$, Miguel Ängel Calleja-Hernández ${ }^{1}$, Wenceslao Aguilera-Morales ${ }^{2}$

\section{${ }^{1}$ FARMACIA, ${ }^{2}$ NEFROLOGÍA, ${ }^{3}$ BIOQUÍMICA, HOSPITAL} UNIVERSITARIO VIRGEN MACARENA, SEVILLA, Spain

Background and Objective: Among the risk factors associated with acute kidney injury (AKI) from prerenal origin, are the use of drugs that exacerbate renal hypoperfusion - nephrotoxic by hemodynamic mechanism - such as NSAIDs and COX-2, ACEI and ARA-II, Cyclosporine and Tacrolimus.

Our objective was to describe the risk factors of patients with AKI from prerenal origin detected through this system, and to analyze the recommendations made by the pharmacist, as well as the evolution of these patients.

Design: Retrospective, single-center study lasting 10 months -from July 2020 to April 2021-, for the selection of patients with AKI in 5 clinical units of our hospital.

A multidisciplinary protocol was established between the Clinical Units of Biochemistry, Nephrology and Pharmacy, using an automated electronic tool for the detection and early action from prerenal AKI (FEUrea $<35 \%$ ), with hospital and Primary Care follow-up.

Upon detecting a case, the pharmacist generated an alert in the electronic prescription system and in the electronic medical record in order to recommend actions on prescribed nephrotoxic drugs, dose adjustments and hydration measures.

Results: 49 cases of AKI from prerenal origin were detected. The distribution by clinical units was: 16 Cardiology, 10 General Surgery, 12 Internal Medicine, 7 Traumatology and 4 Digestive. All patients (49) had extracellular volume depletion as a risk factor, in 36 cases (73\%) a nephrotoxic drug was prescribed: 31 with ACEI / ARB II and 5 with NSAIDs, and in 23 cases (47\%) previous chronic renal failure (GFR $<60 \mathrm{ml} / \mathrm{min}$ ) was founded.

The recommendations made were in relation to the provision of fluids in 47 cases (96\%), suspension of nephrotoxic drugs in 36 cases $(73 \%)$, and dose adjustment in 21 cases $(43 \%)$.

In general, the degree of acceptance of the recommendations was really high, $46(94 \%)$.

Regarding the evolution, most of the cases, 47 patients $(96 \%)$ recovered their renal function in this episode, although in 11 of the 13 cases they died in that episode

Conclusion: There is a high percentage of patients who suffer from prerenal AKI and who are under treatment with this type of nephrotoxic drugs. This suggests that education and empowerment of the patient in relation to this type of medication should be improved to prevent these cases.

We consider that multidisciplinary collaborative work is crucial to improve early action from prerenal AKI in hospitalized patients, in order to optimize the quality of healthcare in these patients.

\section{PP049}

Bioethics considerations, involving mental health patients and pharmacists, in an institution with 330 people

\section{Maria Helena H. Santos ${ }^{*}$, Ana Paula Martins ${ }^{2}$ \\ ${ }^{1}$ Faculdade de Direito de Lisboa, ${ }^{2}$ FFUL, Lisbon, Portugal}

Background and Objective: Mental health disorders and pshychiatric disturbances have a profound impact in people experiencing them, in their families and professional relationships, and in society at large. Even though Mental Health has been acknowledged since 2008 as a Global priority, in many countries, the infrastructures created since them remain suboptimal, in many aspects, such as: number of beds available in pshychiatric wards, specific services available, and number of healthcare professionals specialized in mental health. The contribution of Bioethics is determinant as it emphasises the need to respect patients' autonomy and their vulnerability. The objective of this work is to describe the development of a pharmaceutical care service directed at institutionalised people, living with mental illness. Method: The service was set to serve 330 people and included the identification of areas needing improvement to ensure medicines optimisation. As these areas were developed, a pharmacy team was progressively established, and although the work was strongly anchored in clinical pharmacy, the need to reinforce their education and training in behavioural aspects became obvious (eg. sexuality, stigma, drug use disorders, violence). 
Main outcome measures: n.a.

Results: Some of the areas developed include medicines distribution, creation of medication records, medicines optimisation (including DRP prevention and solution), medication reconciliation, medicines information, collaborative practice, respect for patient confidentiality and privacy, deprescribing, cost minimisation, and patient education. Establishment of links to social care was also necessary for people become de-institutionalized, where the pharmacist ensured onward referral and medicines reconciliation in transitions of care. The pharmacist was prepared to take on its relevant role in clinical pharmacy, being guided by principles such as vulnerability, competence, benevolence, non-malavolence, justice and autonomy.

Conclusion: The ability to provide comprehensive mental health support goes beyond clinical pharmacy. There is a need to understand the bio-psycho-social model in mental health and to deal with concepts of respect for autonomy in people that may be unable to freely make choices. Pharmacists working collaboratively must attain professional accomplishment on the principles and values of Bioethics. There is much work remaining for full attainment of Bioethics in mental health provision.

\section{PP050}

Remdesivir experience in patients with severe SARS-COV-2 infection

Jesus Cotrina Luque ${ }^{1}$, Catia Pereira ${ }^{1}$, Maria José Rei* ${ }^{1}$, Miriam Capoulas $^{1}$, Claudia Santos ${ }^{1}$

${ }^{1}$ Hospital Da Luz Lisboa, Lisboa, Portugal

Background and Objective: Remdesivir is an antiviral and, currently, the only drug approved by the European Medicines Agency for the treatment of SARS-COV-2 in adults and adolescents with severe pneumonia requiring additional oxygen administration.

Our aim was to describe the experience of using remdesivir in a private hospital and evaluate its clinical response as antiviral in patients severally infected with SARS-COV-2.

Design: observational, retrospective study which included patients admitted with SARS-COV-2 infection who received remdesivir between October 2020 and December 2020. Follow up was done until March 2021. All data were obtained by consulting the clinical history and electronic prescription software (SOARIAN ${ }^{\circledR}$ ).

Analyzed variables were demographics (age, sex, days of treatment with remdesivir), efficacy (oxygen saturation at the beginning and at the end of treatment, day of symptoms since beginning of SARSCOV-2 disease, time until recovery defined by either discharge from the hospital or hospitalization for infection-control purposes only, and mortality rate) and safety (reported adverse effects).

Results: 6 patients were included, all male, with a median age of 63 years (Inter Quartile Range, IQR 56-70). 4 of them (67\%) received 5 days of therapy with remdesivir. Median oxygen saturation at the beginning and at the end of the treatment was $90 \%$ and $95 \%$, respectively.

The median time from onset of SARS-COV-2 symptoms to remdesivir administration was

5 days (IQR 4-6). Median time until recovery was 20 days.

Mortality rate at the end of follow-up (median follow-up of 90 days) was $12.5 \%$ (1 patient died).

High levels of alanine aminotransferase (ALT) and pancreatic lipase were reported in 1 patient, resulting in early stopping remdesivir.

Conclusion: Administration of remdesivir itself cannot be associated with clinical improvement of patients with severe SARS-COV-2 infection as median time until recovery was higher than results published on literature (10 days in Adaptive Covid-19 Treatment Trial) but seems to provide a safe choice for treating them.

These results are in line with World Health Organization, which has recently issued a conditional recommendation against the use of remdesivir in hospitalized patients, regardless of disease severity, as there is currently no evidence that this drug improves survival and other outcomes.

\section{PP051}

Use of gene therapy for spinal muscular atrophy: acquired experience in a hospital pharmacy

\section{Maria M. Ferreira ${ }^{*}{ }^{1}$, Miguel Dias ${ }^{1}$, Margarida Queiros ${ }^{1}$, Joana} Ribeiro $^{2}$, Lisete Lemos ${ }^{1}$, Jose Feio ${ }^{1}$

${ }^{1}$ Pharmacy Department, ${ }^{2}$ Child Development Centre, Paediatric Hospital, Coimbra Hospital and Universitary Centre, Coimbra, Portugal

Background and Objective: Describe the circuit of approval, acquisition, preparation and administration of an innovative therapy, based on a clinical case, in a specialized hospital centre.

Design: Chronology of the procedures developed and adopted by the Hospital Pharmacy Department. Process review, based in the use of gene therapy with onasemnogene abeparvovec, in a patient diagnosed with type I spinal muscular atrophy, at the Paediatric Hospital of Coimbra. The evolution of the motor skills was assessed using different medical scales - The Children's Hospital of Philadelphia Infant Test of Neuromuscular Disorders (CHOP-Intend), Hammersmith Neurological Examination part 2 (HINE) and Hammersmith Functional Motor scale expanded (HFMS-E).

Results: In the case presented, all steps were successfully completed both by health professionals and administrative procedures. The clinical status of the patient, nine months after administration of onasemnogene abeparvovec, had a positive response in terms of motor, bulbar and respiratory function, in the acquisition of motor and nutrition skills, as well as in the reduction of the number of respiratory infections. Side effects included transient increase in liver function tests.

Conclusion: Gene therapy is a high-cost therapy under strict preparation, administration and safety measures. The present work discloses the experience and procedures implemented in a Hospital Pharmacy Department. With the involvement of all specialized professionals, it is suggested the elaboration of a consensus document, of the different stages, leading to the use of innovative medicines available for the treatment of spinal muscular atrophy and other orphan diseases.

\section{PP052}

\section{Management of febrile neutropenia in BEP protocol}

Maria N. Rosado* ${ }^{1}$, Ana P. Gomes ${ }^{1}$, Ana Rita Lemos ${ }^{1}$, Andreia Colaço', Miguel Pimenta $^{1}$, Miriam Capoulas ${ }^{1}$, Cláudia Santos ${ }^{1}$

${ }^{1}$ Hospital da Luz Lisboa, Lisbon, Portugal

Background and Objective: Germ cell tumor (GCT) is the most common cancer among young male adults.

Depending on the prognosis and stage of the disease, patients can be treated with 3 or 4 cycles of Bleomycin, Etoposide, Platinum (cisplatin) (BEP)

Febrile neutropenia (FN) is a severe adverse effect of chemotherapy, which frequently occurs in patients under treatment 
with BEP. The aim of this retrospective study is to analyze whether patients doing BEP chemotherapy concomitantly with Granulocyte colony-stimulating factor (G-CSF) (filgrastim or PEG-filgastrim) as primary prophylaxis, developed $\mathrm{FN}$, which risk factors could be involved and compare the efficacy of PEG-filgastrim and filgrastim. Design: This retrospective study was conducted from medical records databases (Soarian ${ }^{\circledR}$ and Farmis Oncofarm ${ }^{\circledR}$ ) of GCT patients treated with BEP from May 2018 to February 2021 at a private hospital in Lisbon. Nine (9) patients had received BEP treatment, however one of them did not receive G-CSF as primary prophylaxis and was excluded. PEG-filgrastim was administered subcutaneously (SC) on day 6 and filgrastim was administered SC from day 8 to day 14. Adverse events, such as leukopenia, neutropenia, FN and others, were analyzed.

Results: Out of 8 patients, 2 patients (25\%) received G-CSF prophylaxis with filgrastim and 6 patients $(75 \%)$ received G-CSF prophylaxis with PEG-filgrastim. During the treatment study, 3 patients $(37.5 \%)$ developed FN. Out of these 3 patients, 2 patients (67\%) did prophylaxis with filgrastim and 1 patient $(33 \%)$ did prophylaxis with PEG-filgrastim. These 3 patients were analyzed concerning CSF administration time (filgastrim vs PEG-filgrastim), the moment they developed FN and the period of time needed to recover standard neutrophils values. Unfortunately, the results were inconclusive. Furthermore, we could identify possible risk factors for $\mathrm{FN}$, such as poor IGCCCG risk classification. There was no additional pulmonary toxicity with concomitantly use of PEG-filgrastim and bleomycin.

Conclusion: PEG-filgrastim administration during BEP treatment can be a potential way to reduce the grade of neutropenia and/or FN with minimal toxicity, improving, at the same time, quality of life. Furthermore, PEG-filgrastim may increase patient adherence and comfort and, consequently a more effective outcome, since it is only necessary one administration of G-CSF instead of various per cycle.

\section{PP053}

COVID-19 and clinical pharmacist interventions-is there an impact?

\author{
Maria Rei" ${ }^{1}$, Ana P. Gomes ${ }^{1}$, Ana R. Silva ${ }^{1}$, Cátia Pereira ${ }^{1}$, Jesús \\ C. Luque ${ }^{1}$, Laura Fernandes ${ }^{1}$, Rita Lemos ${ }^{1}$, Rui Rodrigues ${ }^{1}$, \\ Nazaré Rosado ${ }^{1}$, Miguel Pimenta ${ }^{1}$, Miriam Capoulas ${ }^{1}$, Cláudia \\ Santos ${ }^{1}$ \\ ${ }^{1}$ Pharmacy, Hospital da luz Lisboa, Lisboa, Portugal
}

Background and Objective: COVID-19 affected the day to day work practice of every clinical pharmacist. In this private general hospital, clinical pharmacist interventions (PI) are registered, classified and contribute to several activity indicators, including Performance Quality, Antibiotic Stewardship (ABS) and Patient Safety. We wanted to find out whether last year changes impacted those indicators and PI profile.

Design: A retrospective analysis is conducted considering two 11 month periods, before and during COVID-19 pandemia. We descriptively analyzed the PI in both groups and compared them regarding acceptance rate, communication channels, cause and type of intervention. We also searched the data base for specific COVID 19 interventions involving dexamethasone or remdesivir.

Results: We identified 2434 PI in the pre-COVID period (1 Apr 2019 to 29Feb 2020) and 2996 in the COVID period (1Apr 2020 to $28 \mathrm{Feb}$ 2021). Global acceptance rate was 76 vs $79 \%$ respectively.

The number of PI regarding antibiotics was similar in the two groups as well as their acceptance rate 90 vs $89 \%$ (Pre-Covid vs Covid). The Pharmacist ABS interventions profile also remained stable focusing on pharmacokinetics (PK) and renal dose adjustment interventions in both groups.

Both PI number (532 vs 679) and acceptance rate (66 vs $76 \%$ ) reported to the Risk Management Department increased considerably. In this category we highlight the $40 \%$ increase in the number of PI regarding prescription selection errors or typos.

As anticipated one of the main differences identified regarded the communication channel: $12 \%$ PI done on the wards in the pre-Covid Group vs $0,76 \%$ in the COVID group.

The main causes for PI did not vary depending on being done in a non-COVID or COVID ward. They were: drug dose adjustment based on PK monitoring; alert for prescription duplication and drug dose adjustment for renal impairment.

PI specifically regarding dexamethasone in COVID patients had to do with timing for initiating, excessive treatment duration and oral conversion suggestions. We didn't identify any PI on remdesivir.

Conclusion: Although there were other changes during this period, including the clinical pharmacists team members, that imply caution when looking at this results, the team was able to maintain it's performance as seen by the number of PI and acceptance rate, besides the changes in the context and communication channels that are crucial for PI. The exploration of this analysis also might add to the construction of a checklist for COVID-patient therapeutics validation.

\section{PP054}

Investigation of pharmaceutical care need of COVID-19 patients in hospital settings

\section{Muhammed Yunus Bektay* 1, 2, Mesut Sancar², Fatmanur Okyaltirik $^{3}$, Bulent Durdu ${ }^{4}$, Fikret Vehbi Izzettin ${ }^{1}$}

${ }^{1}$ Clinical Pharmacy, Bezmialem Vakif University, Faculty of Pharmacy, ${ }^{2}$ Clinical Pharmacy, Marmara University Faculty of Pharmacy, ${ }^{3}$ Department of Chest Diseases, ${ }^{4}$ Department of Infectious Diseases and Clinical Microbiology, Bezmialem Vakif University, Faculty of Medicine, Istanbul, Turkey

Background and Objective: Clinical prognosis of COVID-19 may be severe and unexpected. Patients may quickly progress to respiratory failure, infections, multiple organ dysfunction, and sepsis. All these finding revealed the need for pharmaceutical care services for COVID-19 patients. Hence International Pharmaceutical Federation (FIP) suggested pharmacist to join collaborative medical team and provide pharmaceutical services. The main objective of this study is to investigate the pharmaceutical care need of COVID-19 patients.

Method: A descriptive prospective observational was study conducted on COVID-19 patients who admitted to pulmonology service of Bezmialem Vakif University Hospital Istanbul, Turkey. Patient demographics, medications during hospital stay, and history were evaluated. Drug Related Problems (DRP) were identified by a clinical pharmacist. UpToDate ${ }^{\circledR}$ recommendations and guidelines used for classifications, assessment, and identification of DRPs.

Main outcome measures: In this study number of potential drug related problems was main outcomes measures.

Results: Total number of patients were 50, the mean age of patients was $65.6 \pm 15.90$ and $58 \%$ of them were male. Mean score of Body Mass Index (BMI) were 26.9 \pm 4.5 . The median number of comorbidities (min-max) were 3 (1-6) per patient. Majority of patient had at least one comorbidity (94\%) other than COVID-19 and the most frequent comorbidities were hypertension, diabetes, cancer, and COPD recorded in $22,15,10$, and 8 patients, respectively. The mean number of medicine prescribed was $13.2 \pm 6.46$. The most frequently prescribed medicines were favipravir, enoxaparin, pantoprazole, paracetamol, and dexamethasone. The total number of DRP recorded 
as 78 and at least one DRP seen in 36 out of 50 patients. The mean number of DRPs were $1.56 \pm 1.51$ patient. The most frequent DRP was lack of statin use where needed according to STOPP/START criteria and bleeding risk due to concomitant enoxaparin and glucocorticoid use.

Conclusion: In this study many patients had comorbidities and use multiple medications. Besides medication need for COVID-19, medication use for comorbidities such as hypertension and diabetes were required. Considering unknown features of the infection and multiple medication use, DRP are likely to occur. The pharmaceutical care needs of COVID-19 patients increased with comorbidities and multiple medications. Clinical pharmacist should be part of collaborative medical team and provide pharmaceutical care services.

References: 1. World Health Organization (WHO). WHO Coronavirus (COVID-19) Dashboard. Published 2021. https://covid19.who.int/

2. World Health Organization (WHO). Clinical management Clinical management Living guidance COVID-19. World Heal Organ. 2021;(January).

3. International Pharmaceutical Federation F. CORONAVIRUS SARS CoV-2/COVID-19 PANDEMIC: Information and Interim Guidelines for Pharmacists and the Pharmacy Workforce.; 2020. https://www.fip.org/files/content/priority-areas/coronavirus/ Coronavirus-guidance-update-ENGLISH.pdf

4. Cheng F, Li Q, Han Y, et al. Analysis of influencing factors and pharmaceutical care of patients with COVID-19 in Fangcang Shelter Hospital. Infect Drug Resist. 2020;13:3443-3450. doi:10.2147/IDR.S263961

\section{PP055}

Investigation of potential drug-drug interaction in chronic kidney disease patients in nephrology clinic

Aysun Buker ${ }^{1}$, Damla Sosyal ${ }^{2}$, Ozge Ozmen ${ }^{2}$, Muhammed Yunus Bektay $^{* 2,3}$, Meltem Gursu ${ }^{4}$, Rumeyza Kazancioglu ${ }^{4}$, Fikret Vehbi Izzettin ${ }^{2}$

${ }^{1}$ Bezmialem Vakif University, Faculty of Pharmacy, Istanbul, Turkey, ${ }^{2}$ Clinical Pharmacy, Bezmialem Vakif University, Faculty of Pharmacy, ${ }^{3}$ Clinical Pharmacy, Marmara University, Faculty of Pharmacy, ${ }^{4}$ Nephrology, Bezmialem Vakif University, Faculty of Medicine, Istanbul, Turkey

Background and Objective: Chronic kidney disease (CKD) is defined as loss of function or structural damage to the kidney for 3 months or more, irrespective of cause. Due to higher incidence of $\mathrm{CKD}$ and comorbidities in elderly patients, they have more complex treatment regimens. Drug interactions accounts for $20-30 \%$ of all adverse drug reactions. In order to increase treatment success, minimizing drug interactions in patients with CKD is significant. The main objective of this study to evaluate the role of the pharmacist, ensure the adoption of the treatment and the correct use of the drugs by patients, to detect drug interactions.

Method: A cross sectional observational study was conducted in Bezmialem Vakif University Hospital. Etichal approval given by noninvasive Clinical Research Ethics Committee of Bezmialem Vakif University with 21/286 decision number. Patient characteristics, sociodemographics, comorbidites, medicines used and drug-drug interactions were recorded. In this study, we evaluated the drug-drug interactions of patients with chronic kidney disease (GFR $<60 \mathrm{ml} / \mathrm{min} /$ $1.73 \mathrm{~m}^{2}$ ) who applied to nephrology outpatinet clinic. DDIs are evaluated using Lexicomp and Medscape databases.

Main outcome measures: Evaluation of the rate and feature of drugdrug interactions in patients with chronic kidney disease.
Results: 137 patients were included in the study and the mean age of patients was $64.80 \pm 14.59$ years. $41.60 \%$ of these patients were male and $58.40 \%$ female. Patients had CKD for an average of 6.48 years. The mean of number comorbidities was 2.28. The most common of these comorbidities are hypertension, diabetes, and coronary artery disease. 1121 medication usd by patients were evaluated. According to Medscape, 679 drug-drug interactions have been identified. One of them was contraindicated $(0.14 \%), 28(4.12 \%)$ were dangerous and $650(9.72 \%)$ were interventions that require monitor therapy. According to Lexicomp, there were 604 drug-drug interactions. Of these interactions, $9(1.49 \%)$ were in X category, 60 (9.93\%) were in D category and $535(88.57 \%)$ were in C category.

Conclusion: Detection and prevention of drug-drug interactions is important to reduce drug-related problems in patients with CKD. A systematic approach to drug-related problems is recommended, which includes detection, suggestions and interventions of the clinical pharmacist as well as the other healthcare professionals.

References: [1] C. Ponticelli, G. Sala, and R. J. Glassock, "Drug management in the elderly adult with chronic kidney disease: A review for the primary care physician," Mayo Clinic 77 Proceedings. 2015.

[2] "KDIGO 2017 Clinical Practice Guideline Update for the Diagnosis, Evaluation, Prevention, and Treatment of Chronic Kidney Disease-Mineral and Bone Disorder (CKD-MBD)," Kidney Int. Suppl., 2017.

[3] M. Rama, G. Viswanathan, L. D. Acharya, R. P. Attur, P. N. Reddy, and S. V Raghavan, "Assessment of Drug-Drug Interactions among Renal Failure Patients of Nephrology Ward in a South Indian Tertiary Care Hospital.," Indian journal of pharmaceutical sciences. 2012.

\section{PP056}

Pharmacists' perceptions regarding the implementation of automated pharmacy dispensing systems in the United Arab Emirates' hospitals: a qualitative study

Nortan Hashad ${ }^{*}$ 1, 2 , Lamia Al Hajri ${ }^{1}$, Heba Mohamed ${ }^{1}$, Saeed Hussein $^{3,4}$, Waeil Al Naeem ${ }^{5}$, Shamma Alzarooni ${ }^{1}$, Ayesha Almarri $^{1}$, Ayah Ibrahim ${ }^{1}$, Fatma Albanna ${ }^{1}$, Maryam Alattar ${ }^{1}$

${ }^{1}$ Pharmacy, Higher colleges of technology, Dubai, United Arab Emirates, ${ }^{2}$ School of pharmacy and life sciences, Robert Gordon University, Aberdeen, United Kingdom, ${ }^{3}$ Hamdan Bin Mohammed Smart University (HBMSU), Dubai, ${ }^{4}$ Zayed Military Hospital (ZMH), ${ }^{5}$ Sheikh Khalifa Medical City (SKMC), Abu Dhabi, United Arab Emirates

Background and Objective: Automated dispensing systems (ADS) are one of the strategies used in pharmacy to enhance medication management effectiveness and efficiency, hence curb the consequences of medication errors ${ }^{1}$. In the United Arab Emirates (UAE), ADS has been widely implemented, yet pharmacists' perceptions and views regarding ADS and the barriers and facilitators concerning its implementation are not clearly reported in the literature.

The aim of this study is three-folds: (1) explore hospital outpatient pharmacists' perception regarding ADS (2) explore the readiness of pharmacists to embrace the change 3 ) explore the facilitators and barriers towards the implementation of ADS.

Method: A phenomenological qualitative approach was adopted using audio-recorded semi-structured interviews. Participants were recruited purposefully, and snowball sampling was utilized as well. To be eligible, the interviewee should be an outpatient pharmacist in an acute care hospital and have witnessed ADS's pre and post-implementation. The topic guide which was piloted was underpinned by 
the merits of the Consolidated Framework for Implementation Research (CFIR) and surveyed literature. Interviews were transcribed and analyzed independently by two researchers using CFIR ${ }^{2}$.

Main outcome measures: Outcomes included the perspectives and views of participants in relation to ADS implementation in outpatient setting of acute care hospitals in UAE.

Results: Eight interviews (approximately 15-20 minutes) were conducted with outpatient pharmacists from two governmental and two private sector hospitals where the point of saturation was achieved. Several benefits were reported such as; avoiding medication errors, less workload, better time effectiveness and inventory management. It was also found to enhance patient satisfaction by decreasing waiting time and allowing more time for the pharmacist to counsel the patient. Yet, ADS implementation was found to be challenging in terms of financial requirements, structural and infrastructure changes, training and continuous technical support. Facilitators reported are; financial support, leadership and pharmacy staff engagement. Barriers are fear of job loss, lack of pharmacy space to install ADS and no observed benefits in pharmacies with low dispensing rate.

Conclusion: ADS were found to be beneficial especially in facilities with high dispensing rates. Engaging staff and leadership is expected to facilitate implementation allowing more time for the pharmacist to focus on pharmaceutical care and patient safety.

References: 1. Ahtiainen HK, Kallio MM, Airaksinen M, Holmström A. Safety, time and cost evaluation of automated and semi-automated drug distribution systems in hospitals: a systematic review. European journal of hospital pharmacy: science and practice. 2020; 27(5):253262.

2. Damschroder LJ, Aron DC, Keith RE, Kirsh SR, Alexander JA, Lowery JC. Fostering implementation of health services research findings into practice: a consolidated framework for advancing implementation science. Implementation Science: IS. 2009; 4:50-50.

\section{PP057}

\section{COVID-19 - pharmaceutical interventions benefits in adversity times}

\section{Patrícia Santos $^{*}{ }^{1}$, Humberto Melo ${ }^{1}$, Catarina Caçote ${ }^{1}$, Andreia} Loba $^{1}$, Miriam Capoulas', Cláudia Santos ${ }^{1}$

${ }^{1}$ Hospital Pharmacy Services, Hospital Beatriz Ângelo, Lisboa, Portugal

Background and Objective: Our Hospital serves a population of 278000 habitants. Drug dispensing to outpatients is one of the most direct contacts between the pharmacist and the population, it counts with, approximately, 1500 visits/month. With the pandemic's emergency, many patients had difficulties monitoring their disease and accessing their therapy. We were able to increase the patient's adherence to the therapy and their quality of life, through a personalized service and the analysis of our pharmaceutical interventions (PI).

Method: Retrospective analysis of PI data in the context of outpatient drug dispensation, from January 2020 to February 2021.

Main outcome measures: Characterization of the PI performed in the various specialties on an outpatient basis, during the COVID-19 pandemic.

Results: From January 2020 to February 2021, 2261 PI were registered (1055 patients), in various specialties. Ophthalmology had a large increase on dispenses in this period, which is why it is responsible for the biggest increase in the number of PIs (32\%), followed by Infectious Diseases (29\%), Oncology (12\%), Internal Medicine (6\%), Nephrology and Biological Drugs (5\%), Neurology and Gastroenterology (4\%), Pediatrics and Pneumology (2\%). Of all the PI, $71 \%$ were about compliance, followed by $15 \%$ regarding appointments and lab analysis, 3\% to suspend medication, start medication and for changing doses, $2 \%$ to drug interaction, and $1 \%$ due notification of adverse drug reaction, therapeutic duplication, and referral to the emergency department. Several pharmaceutical consultations are held (Infectious Diseases, Oncology, Neurology, Nephrology, Pediatrics, Pneumology and Biological Drugs), and it should be noted that many of these PIs were performed in this context. From all PI, 99\% were Accepted with modification (AwM-prescription/behavior modified as suggested) and $1 \%$ were Accepted without Modification (AoM-Intervention accepted but with justified prescription/behavior maintenance) or were Not Accepted.

Conclusion: In this Pandemic, the patient is faced with some constraints in the follow-up of his disease (difficulty in making appointments, carrying out lab analysis/examinations, the restriction to go to the hospital). However, the pharmacist can play a crucial role in the adherence to the therapy, meeting the needs of patients, proposing solutions, with the ultimate goal of increasing the compliance to the therapy, its efficiency and safety. With this mindset, it was possible to intervene in the various specialties with the common objective to increase the rational drugs use. From all PI, 99\% were AwM, which highlights the importance and relevance of the pharmacist in multidisciplinary teams. This involvement allows hospitalization reduction, prevention of patient readmission and possible adverse effects, thus increasing therapy Compliance, Outcomes and Quality of Life.

\section{PP058}

Intravenous iron formulations: clinical drug appraisal using the SOJA matrix

Roel Fijn* ${ }^{\text {, }}$ Hans C. Ablij ${ }^{2}$, Ad van Bodegraven ${ }^{3}$, Rob Janknegt ${ }^{4}$

${ }^{1}$ Clinical Pharmacy and Toxicology, ${ }^{2}$ Internal Medicine -

Nephrology, Alrijne Healthcare Group, Leiden-Leiderdorp-Alphen aan den Rijn, ${ }^{3}$ Gastroenterology - Hepatology, ${ }^{4}$ Hospital Pharmacy, Zuyderland Medical Centre, Sittard-Geleen, Netherlands

Background and Objective: Iron supplementation is relevant for anaemic patients. When oral administration is ineffective, ill tolerated or otherwise restricted, parenteral administration is indicated. Older intravenous iron formulations involved dextran binding to prevent uncontrolled systemic iron release. However, these were associated with adverse effects. Several alternative safer intravenous formulations have been introduced. However, comprehensive research comparing these formulations in daily clinical practice is unavailable. Our aim was to clinically appraise all available parenteral iron formulations using the System of Objectified Judgement Analysis (SOJA).

Method: SOJA is a structured matrix rationalizing drug selection. Selection criteria are judged by experts. Based on consensus, criteria are given a relative weight. More relevance implies higher relative weighing. For every criterion, ideal properties are agreed on. Each drug formulation is then scored as a percentage of the ideal properties. The highest overall score indicates which drug is most favourable in the actual clinical setting and for formulary inclusion. Analysis included ferric carboxymaltose (Ferinject $\left.{ }^{\circledR}\right)$, ferric dextran complex (CosmoFer $\left.{ }^{\circledR}\right)$, ferric derisomaltose (Monofer ${ }^{\circledR}$, Diafer $\left.{ }^{\circledR}\right)$ and (generic) iron sucrose (Venofer ${ }^{\circledR}$ Ferracin $\left.{ }^{\circledR}\right)$. Ferumoxytol (Rienso $\left.{ }^{\circledR}\right)$ and ferric gluconate (Ferrlicit $\left.{ }^{\circledR}\right)$, presently unavailable, were excluded.

Main outcome measures: Selection criteria were weighed adding up to 1000: (1) licensed indications (60), (2) contraindications (40), (3) warnings and precautions (20), (4) number of dosage presentations 
(60), (5) drug interactions (20), (6) clinical efficacy (300), (7) adverse effects (200), (8) ease of administration (200) and (9) documentation (100).

Results: SOJA scores differed slightly between formulations. Licensed indications, clinical efficacy, drug interactions, and adverse effects scored equally $(60 / 60,210 / 300,18 / 20$ and 160/200). For ease of administration and number of dosage presentations ferric derisomaltose (Monofer ${ }^{\circledR}$, Diafer $\left.{ }^{\circledR}\right)$ scored highest (54/60 and 190/200). Iron sucrose (Venofer ${ }^{\circledR}$ Ferracin $\left.{ }^{\circledR}\right)$ scored lowest $(12 / 60$ and $90 / 200$ ). For contraindications and documentation ferric carboxymaltose (Ferinject $\left.{ }^{\circledR}\right)$ scored highest (32/40 and 100/100). Ferric dextran complex (CosmoFer $\left.{ }^{\circledR}\right)$ scored lowest (20/40 and 83/100). Besides ferric carboxymaltose (Ferinject ${ }^{\circledR}$ ) with a score of $8 / 20$, all other formulations scored $10 / 20$ for warnings and precautions. Overall, ferric derisomaltose (Monofer ${ }^{\circledR}$, Diafer ${ }^{\circledR}$ ) outranked all other formulations with a final score of 830/1000.

Conclusion: Findings indicate that ferric derisomaltose (Monofer ${ }^{\circledR}$, Diafer $\left.{ }^{\circledR}\right)$ favours over other intravenous iron formulations in our actual clinical setting. Using SOJA has provided a transparent and objective matrix for clinical drug appraisal in formulary decisions.

References: 1) Janknegt R, Steenhoek A. The System of Objectified Judgement Analysis. A tool in rational drug selection for formulary purposes. Drugs 1997;53:550-62. 2) Derman R, Roman E, Modiano MR et al. A randomized trial of iron isomaltoside versus iron sucrose in patients with iron deficiency anemia. Am $\mathrm{J}$ Hematol 2017;92:286-291. 3) Onken JE, Bregman DB, Harrington RA et al. A multicenter, randomized, active-controlled study to investigate the efficacy and safety of intravenous ferric carboxymaltose in patients with iron deficiency anemia. Transfusion 2014;54:306-15.

\section{PP060}

Interactions between prescribed medication and chemotherapy

\section{Salvador Ruiz Fuentes* ${ }^{\text {1, Salud Caparrós Romero }}{ }^{1}$, Eva Valverde Alcalá ${ }^{1}$}

${ }^{1}$ Pharmacy, Hospital de la Axarquía, Vélez-Málaga, Spain

Background and Objective: Cancer patients are at great risk of drugdrug interactions which are often not recognized as such as they are masked by symptoms of the pathology itself or assumed to be toxicity inherent to chemotherapeutic agents.Our aim is determining interactions frequency and severity between prescribed medication and chemotherapy drugs prepared in a cytostatic unit.

Method: Retrospective observational study carried out between September and October 2020 in a first level Hospital. All patients who underwent chemotherapy in the cytostatic unit of a first level hospital were included. The variables measured were: age, sex, chemotherapeutic agent, number of prescribed medicines, number and type of interaction. The information sources used were: electronic clinical history and electonic prescription program. It was made a prescribed medication list for each patient and the Lexi-Interact ${ }^{\circledR}$ database was used to confirm the existence of interactions and their severity.

Main outcome measures: It was measured the number of interactions found per patient. They were classified by severity and type of molecule involved.

Results: 84 patients were included (mean age 60.4 years; $53.6 \%$ men, $46.4 \%$ women). $50 \%$ had at least one interaction between chemotherapy and prescribed medication.756 drugs were reviewed, finding an average of 1.4 interactions/patient. Regarding severity, $23.3 \%$ were category B (no action needed), $35.8 \%$ were category C (monitor treatment), $17.5 \%$ were category D (modify regimen) and $23.3 \%$ category $\mathrm{X}$ (avoid combination). The most important ones in terms of frequency and severity (category $\mathrm{X}$ ) were: pyrimidinemetamizole analogues $(32.1 \%)$, platinum-metamizole compounds $(14.3 \%)$, bevacizumab-metamizole $(10.7 \%)$, irinotecan- metamizole (7.1\%), mitomycin-metamizole $(7.1 \%)$ and taxanes-metamizole $(7.1 \%)$.

Conclusion: Half of the patients who are administered chemotherapy have some interaction between them and their usual medication. Implementing detection and prevention programs for these interactions can contribute to improving patient safety.

References: Lexi-Interact ${ }^{\circledR}$ database: https://online.lexi.com

\section{PP061}

Evaluation of a medication reconciliation program at hospital admission in the urology service

\section{Salvador Ruiz Fuentes ${ }^{*}$, , Salud Caparrós Romero ${ }^{1}$, Eva Valverde Alcalá ${ }^{1}$}

\section{${ }^{1}$ Pharmacy, Hospital de la Axarquía, Vélez-Málaga, Spain}

Background and Objective: The continuity of chronic pharmacological treatment during admission to surgical services is a responsibility that has not been fully assumed by any of the professional groups involved in patient care. Medication reconciliation intends to ensure patients receive the necessary medication that they previously took, at the correct dose, route and frequency, appropriate to the current situation and to the new prescription made at hospital. Our aim is determining the incidence and type of reconciliation error (RE), the pharmacotherapeutic groups involved and the degree of acceptance of the interventions carried out by the pharmacist.

Method: Prospective observational study carried out between July and December 2020 at a third level hospital. Polymedicated patients ( 5 drugs or more) admitted to urology were included. The sources of information used were: electronic history, e-prescription program and patient/caregiver interview. Upon admission, a pharmacotherapeutic history was prepared, including a complete list of chronic medication that was compared with that prescribed on admission. Those discrepancies that required clarification were discussed with the urologist.

Main outcome measures: The variables measured were: age, sex, pharmacotherapeutic group (ATC-4), number and type of RE, and acceptance of the pharmaceutical intervention.

Results: 57 patients were included (mean age 73 years; $69 \%$ men, $31 \%$ women). The average number of drugs prescribed per patient was 6.8 and that of RE was 3.4.

$84.2 \%$ of the patients presented at least one RE (97\% omission, $3 \%$ incomplete prescription). $93.5 \%$ of the pharmaceutical interventions were accepted.

The most prescribed ATC-4 groups were proton pump inhibitors (9.3\%), platelet aggregation inhibitors $(6.2 \%)$ and alpha-adrenergic antagonists $(5.3 \%)$. Those most implicated in RE were: ACE-monodrug inhibitors (5.6\%), sulfonamides (4.2\%), and angiotensin-II antagonists and diuretics (3.7\%).

Conclusion: More than $80 \%$ of patients admitted to urology service presented at least one RE, the most frequent being omission. Reconciliation in these patients can help optimize drug therapy during hospitalization. 


\section{PP062}

Medication errors at hospital admission to orthopedic surgery and traumatology service

\section{Salvador Ruiz Fuentes ${ }^{*}$, Salud Caparrós Romero ${ }^{1}$, Eva Valverde Alcalá ${ }^{1}$}

\author{
${ }^{1}$ Pharmacy, Hospital de la Axarquía, Vélez-Málaga, Spain
}

Background and Objective: The design and performance of strategies to improve the quality of health care and patient safety is a priority objective for health authorities. These strategies include medication reconciliation to detect and correct potential medication errors.

The objective of this study is determining the prevalence and type of reconciliation errors (RE) and the pharmacotherapeutic groups involved.

Method: Prospective observational study carried out between January and December 2020 in a first level hospital. Polymedicated patients (5 drugs or more) who were admitted to an orthopedic surgery and trauma service were included.. The sources of information used were: electronic clinical history, electronic prescription program and interview with the patient /caregiver. Upon admission, a pharmacotherapeutic history was prepared, including a complete list of chronic prescribed medication that was compared with that prescribed on admission. Those discrepancies that required clarification were discussed with the responsible physician. To consider a discrepancy as a medication error, the prescriber had to accept it as such. Main outcome measures: The variables measured were: age, sex, prescribed drug, pharmacotherapeutic group (ATC-4), number and type of RE

Results: 421 patients were included (mean age 75.4 years; $47.6 \%$ men, $52.4 \%$ women). The average number of prescribed drugs per patient was 6.7 and the reconciliation errors per patient were 2.96. $84.3 \%$ of the patients presented at least one RE, the most frequent being omission $(90.5 \%)$, different dose-regimen-route of administration $(3.7 \%)$ and unjustified prescription $(1.9 \%)$. The most prescribed ATC-4 groups were HMG-CoA reductase inhibitors (6.5\%), benzodiazepine derivatives $(6.2 \%)$ and mono-drug sulfonamides $(4.2 \%)$. The main groups involved in RE were: benzodiazepine derivatives (7.5\%), HMG-CoA reductase inhibitors $(6.1 \%)$ and adrenergic with corticosteroids $(5.3 \%)$.

Conclusion: The percentage of patients with at least one RE at admission is quite high. The implementation of strategies such as conciliation can help detecting and correcting these potential errors, improving patient safety.

\section{PP063}

Anticholinergic burden in patients treated with acetylcholinesterase inhibitors

\section{Salvador Ruiz Fuentes* ${ }^{1}$, Salud Caparrós Romero', Eva Valverde Alcalá ${ }^{1}$}

${ }^{1}$ Pharmacy, Hospital de la Axarquía, Vélez-Málaga, Spain

Background and Objective: The use of anticholinergic drugs is frequent in older people, even in those with cognitive impairment being treated with acetylcholinesterase inhibitors (AchEIs) for Alzheimer's disease. These drugs produce their pharmacological effect by an opposite mechanism of action resulting in the use of anticholinergics counteracts the effectiveness of AchEIs.

The purpose of this study is determining the anticholinergic burden in patients treated with acetylcholinesterase inhibitors.
Design: Descriptive observational study carried out during 2015 and 2017 in a first level hospital. Patients aged 65 years or older who were admitted to a surgical service and who were being treated with AchEIs for Alzheimer's disease (donepezil, rivastigmine and galantamine) were included. The variables measured were: age, sex, number of drugs prescribed, anticholinergic load according to the score obtained by applying the Drug Burden Index-DBI score, drugs involved. The sources of information used were: electronic clinical history, electronic prescription program and DBI score. On admission, a complete prescribed medication list was obtained and the anticholinergic burden and risk were estimated using the DBI score. Results: 35 patients were included (mean age 82 years; $48 \%$ men, $52 \%$ women) with a mean of 6.2 drugs prescribed per patient. $28.6 \%$ of the patients did not present an anticholinergic burden, $31.4 \%$ presented a low risk, $25.7 \%$ a medium risk and the remaining $14.3 \%$ presented a high risk. The drugs most frequently associated with these adverse effects were: alpha-adrenergic receptor antagonists $(27.1 \%)$, selective serotonin reuptake inhibitors (14.3\%), and benzodiazepine derivatives $(14.3 \%)$.

Conclusion: The anticholinergic burden is moderate or high in a significant percentage of patients treated with AchEIs for Alzheimer's disease. Knowing the prevalence of concomitant prescription of IACE and anticholinergics and identifying affected patients can contribute improving the effectiveness and safety of these treatments.

\section{PP064}

Role of diatrizoate meglumine and diatrizoate sodium solution in the resolution of intestinal obstrution: experience versus authorized indications

\section{Salvador Ruiz Fuentes ${ }^{*}$, Victoria Santana Pareja ${ }^{1}$, Salud Caparrós Romero ${ }^{1}$, Eva Valverde Alcalá ${ }^{1}$}

\section{${ }^{1}$ Pharmacy, Hospital de la Axarquía, Vélez-Málaga, Spain}

Background and Objective: Adhesions are the most important cause of intestinal obstruction, a mechanical bowel condition which prevents the normal movement of the products of digestion. It constitutes about $15 \%$ of hospital admissions for abdominal pain, being a significant cause of hospital mortality with a significant economic cost.Past publications state that Diatrizoate Meglumine and Diatrizoate Sodium Solution (DMDSS), a water-soluble and nephrotropical gastro-intestinal hyperosmolar contrast used only for diagnostic purposes, speeds up the resolution of this situation. However, there is no objective data to support this therapeutic effect.The aim of this study is verifying that intestinal obstruction protocol using DMDSS is safe and allows reducing hospital stay and surgeries due to failure of conservative treatment.

Method: Prospective observational study, following a pre-established protocol.Once the condition is diagnosed, DMDSS is given and conservative treatment is started. If contrast passes to colon at 8,12 or 24 hours post-dose, obstruction is considered partial, oral diet is started and hospital discharge is assessed. If the contrast does not pass at 24 hours, surgery is indicated.They were searched cases from our Hospital treated with $100 \mathrm{ml}$ of DMDSS administered via nasogastric tube or oral through dispensing records from November 2020 to May 2021. We analyzed the evolution of patients decribed at electronic clinal history.

Main outcome measures: The following variables were reviewed: obstruction resolution, need for surgery, complications, andhospital stay.

Results: From November 2020 to May 2021, 17 episodes were treated (15 patients) DMDSS reached the colon in 13 episodes (10 patients), 1 of the patients died after resolving the obstuctive 
condition due to kidney failure and subsequent heart failure. 4 patients were operated, due to poor response to DMDSS protocol and failure of conservative treatment. One of them died as a result of the advanced stage of peritoneal cancer detected during the intervention and the other one due to septic shock, probably secondary to bacterial translocation caused by abdominal distension. The average hospital stay for patients who responded to conservative treatment was 5.7 days whereas it was 11 days for the two patients who were eventually operated and survived. There were no complications related to the administration of DMDSS.

Conclusion: The application of a protocol that includes the administration of DMDSS in the intestinal obstruction caused by adhesions is safe and allows to make therapeutic decisions quicker and therefore, to decrease the hospital stay and the possible complications arising from surgery. Once more, clinical practice and observation proves the is a benefit beyond the authorized indications, however, further studies and close monitoring of the patient are needed.

\section{PP065}

\section{No patient left behind}

Sebastiao Ferreira Da Silva* ${ }^{1}$, Manuela Rodrigues ${ }^{1}$, Rosângela Garcia $^{1}$, Ana P. Dinis ${ }^{1}$, Clara Sequeira ${ }^{1}$, Francisco Machado', José Feio ${ }^{1}$

\section{${ }^{1}$ CHUC, Coimbra, Portugal}

Background and Objective: In 2014, our hospital implemented a drug delivery program to nearby pharmacies (PemProx) chosen by patients, with the objective of increasing the compliance to therapy, improve accessibility to the drugs, increase interaction between pharmacists to increase patient safety and to comply with the NHS goals.

The inclusion of the patients in this program is the pharmcist's responsibility.

The criteria for patient inclusion in PemProx are: Treatment compliance; The impossibility of traveling to the hospital; Therapeutic and disease stability.

As of March 13 2020, 250 patients were included and we had the collaboration of 140 pharmacies in the national territory.

With the declaration of a state of emergency, due to the COVID19 pandemic, in March 2020, PemProx was quickly scaled to respond to the therapeutic needs of patients who were unable to come to our hospital. On March 19 2020, a text message was sent to all our patients, with the following text "COVID 19. Do you need your hospital's medication? Contact one of the numbers 966252479, 966252509. Call and tell us which pharmacy in your area you choose and their information to send the medication"”

A team dedicated to this program was quickly set up to include patients in PemProx by collecting the following data: Patient number; Name; Telephone number; The amount of medication patients still had; Oral consent to participate in PemProx; Pharmacy name; Location; Chief pharmacist; Telephone number.

Then the pharmacies were contacted, through the National Pharmacies Association, via "Operação Luz Verde", to ensure that the shipment was preceded by a patient contact and acceptance.

\section{Design:}

Provide a quantitative description of the impact of this program and the characterization of patients included.

To carry out this analysis we collected to an Excel spreadsheet the data from the contacts we received from the patients to be included in PemProx. We also retrieved the data from prescription filling for the
PemProx patients. The data collected refers to the period from March 13, 2020 to December 31, 2020.

This data was analyzed and arranged by dates, pharmacy (including district) and patient.

Results: From the day the text was sent, on March 20 2020, patients started calling. By September 2020, 5066 different patients had contacted our hospital. Phone calls peaked in the second week after the text was sent.

As of December 31 2020, there were 5083 patients included in PemProxi (53.6\% female) with a mean age of 55.4 years.

During 2020 we sent medicines to 1143 pharmacies throughout the country. The vast majority (445) had only one patient. The average number of patients per pharmacy was 4.4 , with one having 32 patients.

During 2020, 47.775 deliveries were made for patients included in this program.

Conclusion: TThe number of patients who contacted us, who were later included in the program, demonstrates that we have met a population's need. The fact that we have a medication delivery program, still going, through a pharmaceutical circuit guarantees sustainability while complying with good practices.

\section{PP066}

Determination of drug interactions and patient profile in a bone marrow transplantation unit

\section{Gozde Seray Ozturk ${ }^{1}$, Songül Tezcan ${ }^{*}$, Siret Ratip $^{3}$}

${ }^{1}$ Marmara University Institute of Health Sciences, ${ }^{2}$ Marmara University Pharmacy Faculty, ${ }^{3}$ Internal Medicine, Acibadem Hospital, Istanbul, Turkey

Background and Objective: To determine the patient profile and drug interactions in an adult bone marrow transplantation unit (BMT) in a hospital at Turkey.

Method: This was a prospective and descriptive study conducted in an adult bone BMT unit in a private hospital at Turkey between January-June 2021. Patient profile and drug list of the patients were recorded. Drug interactions were analyzed via Medscape drug interaction checker.

Main outcome measures: Determination of patient's sociodemographic characteristics and drug interactions.

Results: Of 23 patients $12(52 \%)$ were female and the mean age was 48. Most of the patients diagnosed with multiple myeloma and $65 \%$ of the patients had at least one comorbid disease (mostly hypertension with $35 \%$ ). While $87 \%$ of the patients were hospitalized for pretransplantation, $83 \%$ of the patients were treated with myeloablative treatment. High dose melphalan regimen was found to be the most administrated regimen (30\%). At least one drug interaction was determined for each patient. Of 152 drug interactions, 98 (64\%) were found as monitored closely and only 1 drug interaction was found to be contraindicated. The mean of the drug interactions was found to be $7.4 \pm 1.6$ in patients with comorbidities. All drug interactions were managed by a multidisciplinary team including the hospital pharmacist.

Conclusion: The number of comorbid diseases was found to be higher in this population. Additionally, drug interactions requiring rationale management and follow-up of the patients were found to be high. We think that clinical pharmacists have an important role in patient monitoring and management of drug interactions in BMT units. 


\section{PP067}

Appropriateness of antiplatelet therapy in combination with anticoagulants in hospitalized patients

\section{Souad Moudallel* $^{1}$, Ferah Ates ${ }^{1}$, Stephane Steurbaut ${ }^{1}$}

${ }^{1}$ Vrije Universiteit Brussel- Universitair Ziekenhuis Brussel, Brussels, Belgium

Background and Objective: Inappropriate concomitant therapy of antiplatelet agents (APAs) and anticoagulants (ACs) entails an increased bleeding risk. The aim was to investigate the inappropriateness rate of the concomitant use of APAs with ACs in the UZ Brussel, a tertiary 729 bed university hospital in Brussels, Belgium. Moreover, pharmaceutical interventions were carried out towards the physicians with documentation of the acceptance and implementation rates of the pharmacists' advices.

Method: A cross-sectional study in hospitalized patients between the 7 th of September and the 17th of November 2020. Descriptive analyses were carried out with SPSS Statistics version 26.

Main outcome measures: Individual chart review was performed to assess the indication of the antiplatelet therapy in patients on anticoagulants according to the summaries of product characteristics and the published guidelines concerning antithrombotic therapy. The second outcome was to assess the acceptance and implementation rates of the pharmacists' advices.

Results: In total, 161 patients were included with a median age of 73 years. The most common encountered antithrombotic combination was acetylsalicylic acid combined with a low molecular weight heparin $(70.8 \% ; \mathrm{n}=114)$ followed by acetylsalicylic acid with a direct oral anticoagulant $(23.6 \% ; \mathrm{n}=38)$. A lack of an indication for the APA use was found in 38 patients $(23.6 \%)$. The physician was contacted by telephone at 31 occasions. There were six patients (3.7\%) for whom the prescribing physician could not be contacted despite several attempts. One patient $(0.6 \%)$ with a suspected inappropriate therapy passed away. The proposal to discontinue the APA use was accepted in $38.7 \%(n=12)$ patients, of which $22.6 \%(n=7)$ were actually implemented.

Conclusion: Approximately 1 in 5 patients received an APA in combination with an AC without any clear indication for the APA. Almost half of the pharmacists' advices to cease the APA were accepted and also implemented for the majority of patients. This study highlights the importance of medication review in patients with concomitant antithrombotic therapy.

\section{PP068}

ASP-intervention by clinical pharmacy in an acute care hospital

\author{
Thomas Arzt ${ }^{*} 1$ \\ ${ }^{1}$ Brothers of Saint John of God Eisenstadt, Eisenstadt, Austria
}

Background and Objective: There is a global trend of growing antibiotic resistance.(1) Infections with resistant bacteria lead to increased patient mortality, patient morbidity, length of hospital stay and pose a great economic burden.(1) Antibiotic overuse is the main trigger for antibiotic resistance. $(2,3)$ Because of high rates of ciprofloxacin und levofloxacin consumption in our hospital, the local multidisciplinary antibiotic stewardship (ASP) team urged for a way of restricting the use of these quinolones. Furthermore the use of orally administered quinolones was underrepresented. Another potential aim was a reduction in the usage of ceftriaxone to tackle the burden of gram-negative resistance. This was proposed, because of the special pharmacokinetics of biliary elimination of ceftriaxone that favour ESBL-induction.(4,5) The pharmacy members of the ASPteam suggested to do a proof of concept study with the aim of reducing quinolone consumption, enforce oral administration whenever quinolone-treatment is necessary and reduce ceftriaxone consumption via recommendations of a clinical pharmacist.

Method: In a time period of three months each treatment with ciprofloxacin, levofloxacin and ceftriaxone on the departments of internal medicine, surgery, traumatology, ENT and neurology was tracked and reviewed by the author of this abstract using an electronic patient record and fever chart. After reviewing the included patient case the clinical pharmacist contacted the physician in charge and discussed possible treatment alternatives. Documentation comprised patient related antibiotic treatment data, the recommendation by the pharmacist and the outcome of the intervention.

Main outcome measures: The main outcome was to reduce consumption of the included antibiotics ciprofloxacin, levofloxacin and ceftriaxone. This was measured in recommended daily doses (RDDs) for each included department. The antibiotic consumption in RDDs of the three month's time period of the study was compared with the exact same time period of the previous year. The second outcome was to measure the rate of acceptance of the pharmacist's recommendations. Therefore each antibiotic treatment recommendation was documented as being accepted, partly accepted or not accepted.

Results: Ciprofloxacin consumption could be reduced by $56 \%$ (505 RDDs in 2019 vs. 220 RDDs in 2020), Levofloxacin by 55\% (500 RDDs in 2019 vs. 226 RDDs in 2020) and Ceftriaxon by $43 \%$ (760 RDDs in 2019 vs. 430 RDDs in 2020). The rate of orally administered Ciprofloxacin stayed the same with $59 \%$. But the rate of orally administered Levofloxacin could be increased from 14\% (2019) to $51 \%$ (2020). In $77 \%$ of the included cases (67 of 87 cases) the clinical pharmacist contacted a physician in charge for an antibiotic treatment recommendation. In 75\% (50 cases) the pharmacist's recommendation was fully accepted, in $10 \%$ ( 7 cases) the recommendation was partly accepted and in 15\% (10 cases) the recommendation was not accepted by a physician.

Conclusion: Clinical pharmacy recommendations are an eligible tool for interventions in the antibiotic treatment setting and can contribute to tackle the global threat of antibiotic resistance on a local scale.

References: (1) World Health Organisation. Sixty-eighth world health assembly. Antimicrobial resistance: Draft global action plan on antimicrobial resistance. Geneva: World Health Organization;2015. (2) Goossens H. Antibiotic consumption and link to resistance. Clinical microbiology and infection: the official publication of the European Society of Clinical Microbiology and Infectious Diseases 2009;15 Suppl 3:12-5. (3) Austin DJ, Kristinsson KG, Anderson RM. The relationship between the volume of antimicrobial consumption in human communities and the frequency of resistance. Proceedings of the National Academy of Sciences of the United States of America 1999;96(3):1152-6. (4) Muller A, Lopez-Lozano JM, Bertrand X, Talon D. Relationship between ceftriaxone use and resistance to thirdgeneration cephalosporins among clinical strains of Enterobacter cloacae. Journal of Antimicrobial Chemotherapy 2004;54:173177. (5) Gray KJ, Wilson LK, Phiri A, Corkill J E, French N et al. Identification and characterization of ceftriaxone resistance and extended-spectrum b-lactamases in Malawian bacteraemic Enterobacteriaceae. 2006;57: 661-665 


\section{PP069}

Evaluation of clinical pharmacy services in pediatric nephrology service

Zeynep Ulku Gun ${ }^{*}$, Nilay Aksoy ${ }^{2}$, Yılmaz Tabel ${ }^{3}$, Mesut SANCAR $^{4}$

${ }^{1}$ Clinical Pharmacy, Inonu University, Malatya, ${ }^{2}$ Clinical Pharmacy, Altinbas University, İstanbul, ${ }^{3}$ Pediatric Nephrology, Inonu University, Malatya, ${ }^{4}$ Clinical Pharmacy, Marmara University, İstanbul, Turkey

Background and Objective: Although there have been some studies on drug-related problems in the general pediatric population, there has been no specialized research on drug-related problems in pediatric nephrology patients. The aim of this study is to provide clinical pharmacy services to hospitalized patients in the pediatric nephrology service, to classify the detected drug-related problems (DRPs) and to determine the clinical pharmacist's contribution.

Method: The study was conducted between April 1st and October 31st, 2019 at a university hospital's pediatric nephrology service. In this process, 66 patients who were hospitalized in this service and using at least 1 drug were included in the study group and DRPs in their treatment protocol was prospectively evaluated according to the European Pharmaceutical Care Network (PCNE v.9). Patients were included in the control group, and their profiles were analyzed retrospectively using data obtained from medical reports for the first 66 patients collected immediately before April 1st. Both of the study group and control group were compared in terms of duration of hospital stay.

Main outcome measures: Accepted intervention percentage and duration of hospital stay were the main outcame measures of our study.

Results: The median age and interquartile range (IQR) values of the 132 patients (66 control group, 66 intervention group) were calculated as 60 months (36-108). In terms of overall demographic and clinical features, both groups were found to be similar $(p>0,05)$. The most commonly used drugs in the treatment of patients in the intervention group are antibacterials $(84,4 \%)$, proton pump inhibitors $(51,5 \%)$ and diuretics $(39,4 \%)$, respectively. A total of 134 DRPs were reported belonging to the intervention group where clinical pharmacy services were given. The most common causes of DRPs were "patient-related" $(36,2 \%)$ in the intervention group. The most common drugrelated problems related to the patient were those under the heading of "inappropriate timing or dosing intervals" $(18,9 \%)$. 96,3\% of the clinical pharmacist's interventions were accepted. It has been demonstrated that presence of polypharmacy and chronic kidney disease both are risk factors for drug-related problems. The intervention group median duration of stay was $7(5-12,5)$ days, while the control group was 9,5 days (7-13) days $(p<0,05)$.

Conclusion: This study demonstrates that clinical pharmacists play an important role in the diagnosis and resolution of DRPs in patients admitted to the pediatric nephrology service, and that clinical pharmacy services can help reduce length of stay.

References: Ibrahim N, Wong IC, Patey S, Tomlin S, Sinha MD, Jani Y. Drug-related problem in children with chronic kidney disease. Pediatr Nephrol. 2013;28(1):25-31

Al Azmi A, Ahmed O, Alhamdan H, AlGarni H, Elzain RM, AlThubaiti RS, Aseeri M, Al Shaikh A. Epidemiology of preventable drug-related problems (DRPs) among hospitalized children at KAMC-Jeddah: a single-institution observation study. Drug Healthc Patient Saf. 2019;11:95-103
PP070

Clinical-epidemiological characteristics of a sprout of klebsiella pneumoniae oxa 48 in a medical-surgical ICU. Analysis of 37 cases

Félix Gómez-De Rueda* 1 , Bárbara C. Díez ${ }^{2}$, Vicente M. Bohorquez $^{3}$, Miguel Angel C. Hernandez ${ }^{3}$

${ }^{1}$ Hospital Pharmacy Unit, Virgen Macarena Universitary Hospital, Seville, ${ }^{2}$ Pharmacy Unit, San Agustin Hospital, Linares (Jaen),

${ }^{3}$ Pharmacy Unit, Virgen Macarena Universitary Hospital, Seville, Spain

Background and Objective: $15 \%$ of hospitalized patients are susceptible to a healthcare-related infection (HRI), especially multiresistant bacteria (MRB) with higher morbidity and mortality, such as Klebsiella pneumoniae Oxa48, responsible for $70 \%$ of infections.

To analyze the clinical and epidemiological characteristics of patients during an outbreak of Klebsiella pneumoniae Oxa48 in the ICU (Intensive Care Unit) of a tertiary hospital in southern Spain.

Design: Retrospective study of 37 patients infected with Klebsiella pneumoniae Oxa48 during September'18-April'21, where histories of infection or colonization by $K$. pneumoniae Oxa 48 were reviewed. Suspicion of colonization was confirmed by rectal swab and infections using scale qSOFA (quick Sepsis-Related Organ Failure Assessment). The variables analyzed were creatinine, leukocytes, lactic acid, respiratory rate, mean arterial pressure (mAP), GGT, GOT, GPT and hemoglobin.

Results: $61 \%$ were men with a mean age of 71 years (63-76) and infection, three were classified as sepsis and one as septic shock (need for vasoactive drugs to maintain $\mathrm{mAP}>65 \mathrm{mmHg}$ ). The average stay was 68.82 days (95\% CI 40.36 to 97.28 ), reaching a maximum value of 183 . The mean values of the main biomarkers were: $\mathrm{Cr}: 1.35 \mathrm{mg} / \mathrm{dl}$ (95\% CI 0.93 to 1.54$)$, leukocytes: $18.6 \times 109 / \mathrm{L}, \mathrm{Hb}: 9.42 \mathrm{~g} / \mathrm{dL}(95 \%$ CI 7.42 to 11.3 ), lactic acid: $4.16 \mathrm{mmol} / \mathrm{L}$ (95\% CI 1.41 to 6,80$)$, GGT: 845.1 U/L (95\% CI 554.6 to 1090.7), GOT: 244.2 U/L (95\% CI 119.08 to 360,7$)$ and GPT: $133.6 \mathrm{U} / \mathrm{L}$ (95\% CI 41.65 to 204.34). Both colonized/infected patients tested positive for Klebsiella pneumoniae Oxa48, with an average value of days from admission to positive cultures of 21,9 days (95\% CI 13.21 to 35,6 ). Twenty two patients were sensitive to Ceftazidime/Avibactam $(2 \mathrm{~g} / 0.5 \mathrm{~g} / 8 \mathrm{~h}) .67 \%$ had to adjust doses due to renal function. $78 \%$ were exitus. Fourteen patients were positive for CoVid'19, although only five had an asymptomatic situation.

These data correspond to the extension of a previous analysis prior to CoVid'19, so data related to this infection are not included.

Conclusion: The MRB severely condition the clinical situation of patients, prolong hospital admissions and are associated with high morbidity and mortality.

It is essential to comply with hygienic-preventive measures by both professionals and family members, to prevent the spread of germs and colonization of new patients.

\section{PP071}

Manipulation of parenteral nutrition bags with marketing authorization in pediatrics: clinical audit of practices Marie GUEDON ${ }^{*}{ }^{1}$, Catherine MENNESSON ${ }^{1}$, Coralie
BOULANGER $^{1}$, Dominique HETTLER

${ }^{1}$ CHU de Reims, REIMS, France 
Background and Objective: The handling of parenteral nutrition bags is a risky step in the management of patients in neonatology and pediatric intensive care. This risk has been evaluated and is the subject of new recommendations established by the Inspection Générale des Affaires Sociales (2015), the Haute Autorité de Santé (2018), the Société Française d'Hygiène Hospitalière (2020) and the Agence Nationale de Sécurité du Médicament et des Produits de Santé (2020). This study is part of a quality approach and aims to evaluate the application of these recommendations within the care units and to estimate the training needs of the nursing staff.

Design: A prospective observational study of professional practices in pediatric care services (neonatal and pediatric intensive care units, pediatric surgery, pediatric medicine, and pediatric oncology hospitalization) was carried out. With the support of studies carried out in other centers, an audit grid was drawn up, covering various recommendations made. Three main areas are covered: bag reconstitution, supplementation conditions and bag administration.

Results: Thirteen assessments were performed over a six-week period by the health executives and nurses. No observations could be made in the Onco-Pediatric Hospitalization Unit (no prescription during the period). This audit showed that the reconstitution stage was compliant ( $100 \%$ compliance, $n=13)$ in all the departments audited. Regarding supplementation, no supplementation was performed in newborns. The supplements in infants mainly concern vitamins $(n=6)$ and oligoelements $(n=2)$ (added in the bag) and lipids $(n=3)$ (added in Y). In one department, two selenium supplementations were carried out as well as an injection of iron in Y (with rinsing of the tubing before and after the drug was administered). Finally, concerning the administration of the bag, there was no photoprotection ( $0 \%$ compliance) or terminal anti-particulate filter (33\% compliance, $\mathrm{n}=4)$. The traceability steps (patient file and labels) were satisfactory $(92 \%$ compliance, $\mathrm{n}=11$ ).

Conclusion: This study provided an overview of the practices between the different pediatric services of the hospital. If certain handling rules, such as reconstitution of bags, are fully understood by the nursing staff, there are still points to improve. These non-compliances according to the guidelines mainly concern the administration of the bags. The results allow us to implement focused training on the latest recommendations and particularly on the conditions of supplementation and bag administration. E-learning enables the continuous training of nurses, within the care service, and is therefore easily flexible depending on the evolution of the recommendations. This training is completed by an institutional procedure to harmonize practices within the various pediatric services.

\section{PP072}

Assessing the effects of a psychiatric PharmD rotation on mental health knowledge and attitudes toward the provision of pharmaceutical care to people with mental illness

\section{Monica Zolezzi* ${ }^{1}$, Rawan Ghanem ${ }^{1}$, Maryam Swillam ${ }^{1}$, Bridget Javed $^{1}$}

\section{${ }^{1}$ College of Pharmacy, QU Health, Qatar University, Doha, Qatar}

Background and Objective: Pharmacists can have a positive impact when caring for people with mental illness. However, multiple survey studies have exposed that pharmacists are less willing to provide pharmaceutical care to patients with mental illnesses than to those with medical illnesses. Experiential education helps to address these stigmatizing attitudes. Given the apparent importance of patient contact in changing pharmacy students' attitudes toward mental illness, this study aims not only at assessing the effectiveness of the psychiatric pharmacy PharmD rotation on the students' knowledge, but also in regards to how this clinical exposure impacts their attitudes and beliefs surrounding mental health care provision in practice.

Design: This is a two-phase study, consisting of an initial retrospective review of pre/post-rotation questionnaires administered to the PharmD graduates during their psychiatric rotations, followed by semi-structured interviews that explored their views about the psychiatric rotation. In the second phase, all PharmD graduates, regardless if they had a psychiatric rotation during their PharmD studies, were surveyed to evaluate their attitudes and beliefs about the provision of pharmaceutical care to people with mentally illness.

Results: The analysis of the pre and post questionnaires showed that there was a significant improvement in the students' overall knowledge of mental illnesses and on the pharmacotherapy of these conditions. Additionally, PharmD students were more comfortable in performing clinical services to mentally ill patients after they had undergone a psychiatric rotation. Thematic analysis of the interviews resulted in 5 major themes: familiarity with mental illness, expectations of the mental health rotation, understanding of mental illness stigma, barriers to the psychiatric rotation experience, and learning opportunities of mental health rotation. A total of 23 PharmD graduates responded to the survey ( $27 \%$ response rate) of which close to $50 \%$ completed a psychiatric rotation during their PharmD program. Overall, no significant difference was observed in the attitudes and beliefs of PharmD graduates exposed or not exposed to a psychiatric rotation in regards to stigma towards mental illness or towards the provision of pharmaceutical care to patients with a psychiatric condition.

Conclusion: The psychiatric PharmD rotation improves future pharmacists' overall knowledge and beliefs regarding mental illnesses and appear to positively influence their attitudes towards the provision of pharmaceutical care to people with mental illness.

\section{PP073}

The role of the clinical pharmacist in detecting drug interactions and drug-related problems in patients with lung and urogenital system cancer

Selma Sezer* ${ }^{1}$, Miray Akbaş ${ }^{1}$, Begüm Sanat Dayı ${ }^{1}$, Zeliha Pala Kara $^{2}$, Meltem Ekenel ${ }^{3}$, Birsel Sönmez Uydeş Doğan ${ }^{2}$

${ }^{1}$ Clinical Pharmacy, ${ }^{2}$ Pharmacology, ${ }^{3}$ Medical oncology, Istanbul University, İstanbul, Turkey

Background and Objective: Complex prescription regimens and comorbidities may cause drug-related problems (DRPs) and significant drug-drug interactions in cancer patients.

To verify possible drug-drug interactions and DRPs in patients with genitourinary system or lung cancer.

Method: This study was carried out retrospectively in between 08.02.2020 and 02.04.2020 at Istanbul University Institute of Oncology. Drug interactions were identified by using IBM Micromedex Drug Interaction programme and were classified according to their severity as either contraindicated, major, moderate, and minor interactions. The identifed problems were recorded according to Pharmaceutical Care Network Europe V9.00 (PCNE) classification. Main outcome measures: A total of 142 patients, 74 (52.1\%) with genitourinary system and $68(47.9 \%)$ lung cancer, were included in the study. Median age was 59.5 years-old and female to male ratio was $69 / 73$. The median number of drugs per patient was 13.4. A total of 739 drug interactions were detected in $122(85.9 \%)$ patients. Of 
those interations, $0.1 \%$ were contraindicated, $61.4 \%$ major, $33.7 \%$ moderate and $4.7 \%$ minor.

Results: Concomitant use of Dexamethasone-Diclofenac detected in 26 patients was the most common interaction. Total 163 DRPs were detected in $83(58.5 \%)$ patients. According to PCNE classification, the most common problems were adverse drug event (possibly) occurring $(n=141)$ and effect of pharmacotherapy $(n=22)$. Main cause of the problems (83\% of total) was inappropriate drug combinations. Conclusion: Cancer patients are involved in risky group of patients in terms of drug interactions and DRPs. Identifying these problems and raising awareness by clinical pharmacists will provide positive results for both patients and healthcare professionals.

\section{PP074}

Clinical impact of factors that modify infliximab pharmacokinetics

\section{Adriana Moreno Herrera ${ }^{1}$, Alberto Soria Martin ${ }^{1}$, Sol P. Cortes de Miguel $^{1}$, Javier Garcia Marin* ${ }^{*}$, Marina Fages Perez ${ }^{1}$ \\ ${ }^{1}$ FARMACIA, HOSPITAL PUNTA DE EUROPA, ALGECIRAS, Spain}

Background and Objective: To determine the impact of factors related to the pharmacokinetics of infliximab in patients with Inflammatory Bowel Disease (IBD)

Design: Retrospective descriptive study, which included patients with IBD who were asked for infliximab plasma levels between September 2019 and March 2021, both included. The data were obtained from the review of medical and laboratory records in Diraya. The interval established as therapeutic was $3-7 \mathrm{mcg} / \mathrm{ml}$. The analytical technique used was ELISA. Anti-infliximab antibody values $>10 \mathrm{U} / \mathrm{ml}$ were considered positive. The values that determined active disease were: Harvey Bradshaw Index $>6$ in Crohn's Disease (CD), and Mayo Clinic Activity Index $>9$ in Ulcerative Colitis (UC). Factors that affect the pharmacokinetics of infliximab were considered: male sex, type of disease (being higher in UC than in CD), low levels of albumin, high levels of C-reactive protein (CRP), presence of antibodies and non-use of immunomodulatory drugs (corticosteroids, mesalazine and azathioprine) concomitantly

Results: 23 patients were included, 14 men and 9 women, with a mean age of 42 years, 22 with CD and 1 with UC. No patient had active disease at the time of the study. 11 patients presented infliximab infratherapeutic levels, of which 7 were men and 4 women; 1 had UC and 10 had CD. Regarding albumin, 5 showed low levels, and of those, 4 had increased clearance. Only 11 patients had plasma CRP levels determined. Of those 11, 2 showed elevated CRP levels and one of them had infliximab infratherapeutic levels. 18 patients were tested for antibody levels, 2 had positive levels, and clearance was increased in both. Finally, of the 19 patients who were receiving concomitant immunomodulator therapy, 10 had infliximab infratherapeutic levels

Conclusion: In our sample, the factors that seem to influence an increase in infliximab plasma clearance are: male sex, low albumin concentrations, and the presence of anti-infliximab antibodies. However, we can not establish the influence of high CRP levels due to lack of analytical determinations. We also do not have a clear correlation with the concomitant use of immunomodulators and an infratherapeutic level of infliximab

\section{PP075}

Clinical impact of factors that modify adalimumab pharmacokinetics

Adriana Moreno Herrera ${ }^{1}$, Alberto Soria Martin ${ }^{1}$, Marina Fages Perez $^{1}$, Javier Garcia Marin ${ }^{*}$, Javier Romero Puerto ${ }^{2}$

${ }^{1}$ FARMACIA, HOSPITAL PUNTA DE EUROPA, ALGECIRAS, ${ }^{2}$ FARMACIA, HOSPITAL NAVALMORAL DE LA MATA, CACERES, Spain

Background and Objective: To determine the impact of factors related to the pharmacokinetics of adalimumab in patients with Inflammatory Bowel Disease (IBD)

Design: Retrospective descriptive study, which included patients with IBD who were asked for adalimumab plasma levels between September 2019 and March 2021, both included. The data were obtained from the review of medical and laboratory records in Diraya. The interval established as therapeutic were $7.5-12 \mathrm{mg} / 1$ for Crohn's Disease (CD) and 3-10 mg/l in Ulcerative Colitis (UC). In both cases, the analytical technique used was ELISA. Anti-adalimumab antibody values $>10 \mathrm{U} / \mathrm{ml}$ were considered positive. The values that determined active disease were: Harvey Bradshaw Index $>6$ in $\mathrm{CD}$ and Mayo Clinic Activity Index $>9$ in UC. Factors that affect the pharmacokinetics of adalimumab were considered: male sex, type of disease (being higher in UC than in CD), low levels of albumin, high levels of C-reactive protein (CRP), presence of antibodies and nonuse of immunomodulatory drugs (corticosteroids, mesalazine and azathioprine) concomitantly.

Results: 32 patients were included, 16 men and 6 women, with a mean age of 38,6 years, 26 with $\mathrm{CD}$ and 6 with UC. 11 patients had active disease at the time of the study. 10 patients presented adalimumab infratherapeutic levels, of which 3 were men and 7 women; 1 had UC and 9 had CD. Regarding albumin, 3 showed low levels, and of those, 1 had increased clearance. Only 19 patients had plasma CRP levels determined. Of those 19, 8 showed elevated CRP levels and 6 of them had increased clearance. 12 patients were tested for antibody levels, 1 had positive levels and this one had increased clearance.

Finally, of the 23 patients who were receiving concomitant immunomodulator therapy, 5 had adalimumab infratherapeutic levels. Conclusion: In our sample, the factors that seem to influence an increase in adalimumab plasma clearance are: high CRP levels, the presence of anti-infliximab antibodies and the concomitant use of immunomodulators. However, we cannot establish a clear correlation between sex, type of disease, and low albumin concentrations with an infratherapeutic level of adalimumab

\section{PP076}

Transdermal to oral selegiline monitored switch in a 62 year old woman with resistant depression

Matthieu Lebrat ${ }^{*}$, Jonathan Boisrame ${ }^{1}$, Filipe Galvao², Hugo Turbe $^{2}$, Antony Citterio-Quentin ${ }^{3}$, Bertrand Clerc ${ }^{1}$

${ }^{1}$ Department of Pharmacy, ${ }^{2}$ Department of Psychiatry, Le Vinatier Hospital, Psychiatric Hospital, Bron, ${ }^{3}$ Department of Biology, Hospices Civils de Lyon, Lyon, France

Background and Objective: To present and document a drug monitored off-label galenic switch of selegiline in a resistant depression where the transdermal patch could not be used anymore due to skin reaction.

Design: Case report of the selegiline patch to oral switch, monitored with blood concentration of the selegiline and its metabolites with 
dose adjustment. Concomitant clinical monitoring and close collaboration with the psychiatrist, clinical pharmacists and biologist.

Results: A literature review was first carried out on the use of oral selegiline in the depression treatment. We then developed a dosage method specially for our patient's switch. An initial reference concentration of selegiline and its metabolites under the transdermal selegiline form $(12 \mathrm{mg} / 24 \mathrm{~h})$ was assessed by 2 blood samples analysis. Then a switch has been undertaken with oral selegiline regarding the benefit/risk ratio in favor of this switch in this resistant depression that solely responded to selegiline. The initial concentrations under the oral form at $10 \mathrm{mg}$ daily didn't reach the transdermal ones so a concentration monitored posology increase has been done until $20 \mathrm{mg}$ daily. The selegiline concentration then reached $0.90 \mu \mathrm{g} / \mathrm{L}$ vs. 5.32$5.44 \mu \mathrm{g} / \mathrm{L}$ with the patch whereas the metabolites concentrations of L-methamphetamine and L-amphetamine were twice that of the patch. A fourth posology increase has nonetheless not been undertaken due to high levels of amphetamine metabolites raising safety concerns and the treatment has therefore been stopped after 9 weeks. Conclusion: This monitoring with 5 blood titrations confirmed the different pharmacokinetics of the 2 forms and rose the question of L-amphetamine psychoactivity due to its significant concentration even though this isomer is less active. The difficult therapeutic management of treatment-resistant depression and the occurrence of an adverse reaction with the transdermal form has been in favor of oral selegiline use in our case. The drug monitoring that we have set up allowed a controlled galenic switch that didn't reach the expected therapeutical effects for our patient despite a posology increase with good tolerability.

\section{PP077}

Clozapine therapeutic monitoring: promoting effectiveness and safety in psychiatric patients

\section{PEDRO Cardoso ${ }^{1}$, EUGÉNIA Marques ${ }^{1}$, INÊS Rosário ${ }^{2}$, INÊS Pereira $^{2}$, MIRIAM Capoulas ${ }^{1}$, HELENA Cordeiro ${ }^{1}$, CLÁUDIA Santos ${ }^{1}$}

${ }^{1}$ Hospital Beatriz Ângelo, Loures, ${ }^{2}$ Faculty of Pharmacy Lisbon, Lisboa, Portugal

Background and Objective: Therapeutic Drug Monitoring (TDM) in psychiatry is critical due to a significant impact of interindividual variability on the treatment effectiveness and to control drug related problems (DRP). Clozapine (CZP) is a widely used atypical antipsychotic, especially in the treatment of refractory schizophrenia and psychosis. CZP concentrations in blood are predictive for relapses and TDM may reduce the risk of relapse or recurrence. According to European consensus guidelines of TDM in Neuropsychopharmacology, CZP range in blood is $350-600 \mathrm{ng} / \mathrm{ml}$. This study evaluates CZP blood levels in our patients, the occurrence of DRPs and hospital staying duration.

Design: This is a retrospective observational study of psychiatric inpatients and outpatients in our centre, between 2012 and 2021. Data was collected from the Electronic Health Records. Features collected include sex, age, diagnosis, date of dosing, daily dosage and length of hospital stay for inpatients. Statistical analysis was performed using Python 3.8.8 and Microsoft Excel 2019.

Results: The study included 181 patients, the majority being males $(56 \%)$. The median age of the sample was 45 years (17-84) and 55\% were outpatients. Schizophrenia was the most prevalent diagnosis (51\%), followed by Non Otherwise Specified Psychosis (NOSP) and Bipolar Disorder (11\%). The analysis included 581 CZP measurements. There was no significant correlation between daily dosage and blood level (pearson $=0.26$ ). Only $20 \%$ of TDMs were between the adequate range (median $250 \mathrm{ng} / \mathrm{ml}, 3-2511$ ), $61 \%$ of patients had subtherapeutic levels of CZP and $19 \%$ presented supratherapeutic levels. The length of hospital stay was bigger for patients with levels higher than recommended, average of 169 days vs 84 days $(\mathrm{p}<0.05)$. Schizophrenic and patients with NOSP had lower levels of CPZ comparing to other diagnosis $(\mathrm{p}<0.01)$. Drug Related Problems were present in 23 patients, mainly sialorrhea (52\%), constipation (17\%) and extrapyramidal effects (13\%).

Conclusion: Despite the importance of maintaining adequate levels of CZP, the majority of psychiatric patients still present blood levels outside the recommended range. Pharmacists should recommend and evaluate TDM of antipsychotics to promote safety and increase effectiveness of treatment.

\section{PP078}

The impact of monitoring FVIII levels in clinical practice for hemophilia A

Raquel Pinho* 1 , Marília J. Rocha ${ }^{2}$, Francisco Machado², Ramon Salvado $^{3}$, Ana Fortuna ${ }^{1,4}$

${ }^{1}$ Laboratory of Pharmacology, Faculty of Pharmacy of University of Coimbra, ${ }^{2}$ Pharmaceutical Service, ${ }^{3}$ Congenital Coagulopathies Center, University Hospital Centre of Coimbra, ${ }^{4}$ CIBIT/ICNAS, Coimbra Institute for Biomedical Imaging and Translational Research, Coimbra, Portugal

Background and Objective: Monitoring FVIII levels is crucial for a safer and effective treatment in Hemophilia A (HA $)^{[1]}$. Replacement treatment with clotting factor concentrates (CFCs) may be assessed through a minimum of three samples ${ }^{[2]}$ using the trough levels, at least, above the limit $\left(\mathrm{C}_{\text {trough }}>1 \mathrm{IU} / \mathrm{dL}\right)$ as the main outcome to prevent clinical complications and reverse the severe state into a milder one ${ }^{[3]}$. The main aim was evaluating the monitoring of HA patients followed at the Immuno-Hemotherapy of the University Hospital Centre of Coimbra (CHUC)

Method: This was a retrospective study in HA patients $(>17$ years old) followed at the Immuno-Hemotherapy of the University Hospital Centre of Coimbra (CHUC; EPE) between January 2018 to June 2020, with at least least one CFCs prescription. The study enrolled 46 patients with all of the demographic, clinical and treatment data collected in Excel and analysed alongside with SPSS program for statistics.

Main outcome measures: The main outcome was the $\mathrm{C}_{\text {trough }}$ values related to the blood sampling. Alongside them, it was taking into account the severity of the patients, the regimen (prophylaxis /ondemand) and the clinical outcomes seen with that monitioring.

Results: This study enrolled 46 patients with mean of age 43.7 years $( \pm 2.2$ ) with at least one CFCs prescription. Overall, most of the patients $(87 \%)$ had their levels monitored. However, $45.7 \%$ of them had only one blood sampling to be analysed to determine the FVIII levels. Among them, 21.7\% $(n=10)$ were severe HA patients within prophylactic regimen (group1) and $15.2 \%(n=7)$ were severe patients but on-demand (group 2). Twelve patients had a $\mathrm{C}_{\text {trough }}<1 \mathrm{IU} / \mathrm{dL}$ ( $n=4$ of the group $1 ; n=7$ in group 2 ) which explains the hemorrhages, knees replacement surgery or, worse, the development of ankle hemarthroses, verified in regardless the regimen.

Conclusion: Even though several studies and guidelines recommend monitoring HA treatment for efficacy and safety, these results are the reflection that this field needs an established protocol in clinical practice to give the patients a better clinical outcome.

References:1. DELAVENNE, Xavier; DARGAUD, Yesim - Pharmacokinetics for haemophilia treaters: Meaning of PK parameters, interpretation pitfalls, and use in the clinic. Thrombosis Research. ISSN 18792472. 192 : May (2020) 52-60. doi: 10.1016/ j.thromres.2020.05.005. 
2. BJÖRKMAN, S.; COLLINS, P. - Measurement of factor VIII pharmacokinetics in routine clinical practice. Journal of Thrombosis and Haemostasis. ISSN 15387933. 11:1 (2013) 180-182. doi: 10.1111/jth.12055.

3. COLLINS, P. W. et al. - Implications of coagulation factor VIII and IX pharmacokinetics in the prophylactic treatment of haemophilia. Haemophilia. ISSN 13518216. 17:1 (2011) 2-10. doi: 10.1111/j.1365-2516.2010.02370.x.

\section{PP079}

Is the pharmacokinetics of perampanel influenced by gender and comedication in refractory epileptic patients?

RUI Silva $^{*}$ 1, 2, LUÍS Fonseca ${ }^{1,2}$, ANABELA Almeida ${ }^{1,3}$, JOANA Bicker $^{1,}$, , FRANCISCO Sales ${ }^{4}$, AMÍLCAR Falcão ${ }^{1,}$, ANA Fortuna ${ }^{1,2}$

${ }^{1}$ CIBIT - Coimbra Institute for Biomedical Imaging and Tanslational Research, ${ }^{2}$ Faculty of Pharmacy, University of Coimbra, ${ }^{3} \mathrm{CIVG}$ Centro de Investigação Vasco da Gama, Escola Universitária Vasco da Gama, ${ }^{4}$ Refractory Epilepsy Reference Centre, Centro Hospitalar e Universitário de Coimbra, EPE, Coimbra, Portugal

Background and Objective: Perampanel (PER) is a third-generation antiepileptic drug approved for the adjunctive treatment of partialonset seizures and primary generalized tonic-clonic seizures in patients with idiopathic generalized epilepsy. It is the only antiepileptic drug that decreases the excitatory transmission by selectively inhibiting the postsynaptic $\alpha$-amino-3-hydroxy-5-methyl4-isoxazolepropionic acid (AMPA) receptor. Moreover, PER is completely absorbed and have a long half-life which allows oncedaily administration. However, it binds to plasma proteins in an extent of $96 \%$ and is metabolized by hepatic CYP450 enzymes, increasing the potential to develop drug-drug interactions. This study aimed at characterizing the pharmacokinetics of PER in adult Portuguese refractory epileptic patients.

Method: A retrospective study was performed including 22 epileptic patients (12 males and 10 females, age mean: $37.9 \pm 14.6$ years) admitted to the Centro de Referência de Epilepsia Refratária of Centro Hospitalar e Universitário de Coimbra and treated with PER. Blood samples were collected before drug administration at bedtime and on the morning of the following day to quantify PER. Demographic, analytical, and co-administered drugs were collected. A total of 44 plasma concentrations were used to estimate the volume of distribution (Vd) and the clearance (CL) of PER for each patient, resorting to a one-compartment model with first-order absorption and elimination and applying the Abbottbase PKS software.

Main outcome measures: Estimation of the pharmacokinetic parameters of PER and evaluation of demographic variables and comedication effect, including the influence of CYP-inducing antiepileptic drugs.

Results: The most frequently administrated doses were $8 \mathrm{mg}(8$ patients, 36.4\%) and $6 \mathrm{mg}$ (7 patients, 31.8\%). The median and $25 \%$ and $75 \%$ quartile values of the pharmacokinetic parameters of PER observed in the study population were: $0.376(0.281-0.526) \mathrm{L} / \mathrm{kg}$ for $\mathrm{Vd}$; and $0.0085(0.0058-0.0130) \mathrm{L} / \mathrm{h} / \mathrm{kg}$ for CL. The half-life found was 31.48 (19.89-50.05) h. Statistically significant differences were observed between the CL of patients taking carbamazepine $[0.0135$ $(0.0105-0.1930] \mathrm{L} / \mathrm{h} / \mathrm{kg}]$ with that observed in patients not submitted to this CYP-inducing drug [0.0060 $(0.0050-0.0098) \mathrm{L} / \mathrm{h} / \mathrm{kg}]$. The observed half-life was $55,8 \%$ lower in patients taking carbamazepine.
Conclusion: Co-administration of carbamazepine enhances the clearance of PER and consequently reduces its half-life, suggesting that posology of perampanel may need dose optimization, in order to achieve the optimal therapeutic effect.

Acknowledges: This work was financed by FEDER funds through Portugal 2020 in the scope of the Operational Programme for Competitiveness and Internationalization, and Fundação para a Ciência e Tecnologia (FCT), Portuguese Agency for Scientific Research, within the scope of the research project POCI-01-0145-FEDER-030478

\section{PP080}

LC-MS/MS application for therapeutic drug monitoring for both TKIS and tri-azole antifungals in Chinese leukemia patients

\section{Wen Ting Zhang ${ }^{*}$, Yang Zhang ${ }^{1}$, Dong Liu ${ }^{1}$}

${ }^{1}$ Department of Pharmacy, Tongji Hospital of Tongji Medical College, Huazhong University of Science \& Technology, Wuhan, China

Background and Objective: Due to the high risks of IFIs, leukemia patients on chronic TKIs therapy are commonly co-prescribed with antifungal agents during induction, consolidation, hematopoietic stem cell transplantation, and maintenance therapy. But there is a poor correlation between the oral voriconazole dose and plasma concentrations, and many patients achieve levels that are considered to be subtherapeutic. Moreover, tri-azole antifungals are strong CYP3A4 inhibitors that may result in significantly increased plasma levels of TKIs and thereby increased risks of toxicities from TKIs. Therefore, routine therapeutic drug monitoring (TDM) and appropriate doseadjustment for both tri-azole antifungals and TKIs are critical for antileukemia and antifungal therapeutics in these patients. Herein, an rapid LC-MS/MS method for simultaneous determination of three TKIs and two tri-azole antifungal agents in one blood draw was successfully applied to routine TDM for leukemia patients. Method: Leukemia patients on concurrent TKI and tri-azole antifungal therapies were asked to take the TKI and antifungal agent at the same time of the day. With informed consent, plasma samples were collected just before (within $0.5 \mathrm{~h}$ ) the next dose at the steadystate (at least 7 days into both TKI and antifungal regimen) and analyzed within established stability time frame. Patients' medical records were retrospectively reviewed to collect clinical information. Main outcome measures: The ratio values of $\mathrm{C}_{\min }(\mathrm{ng} / \mathrm{mL})$ and Daily dosage $(\mathrm{mg})$ represented blood concentration of imatinib per unit dose. Comparison of the ratio between two groups (imatinib with or without voriconazole/ itraconazole) were evaluated by the unpaired Student's t test. Data were expressed as means \pm SD. Differences were considered significant at $P<0.05$.

Results: Compared with the 27 leukemia patients (41 plasma samples) on imatinib without voriconazole/ itraconazole, the 12 leukemia patients (16 plasma samples) with combination use of voriconazole/ itraconazole presented higher blood concentration of imatinib per unit dose $($ mean $=5.24)$ and greater inter- and intra-individual variation $(\mathrm{SD}=4.27)$.

Conclusion: Due to greater individual variation, close monitoring for toxicity and dosage adjustments of TKIs for leukemia patients who are concomitantly administered with triazole antifungals should be considered. TDM using the method, combined with careful clinical evaluations, can be helpful to improve treatment success, decrease adverse drug reactions, and save drug costs for these patients. 


\section{PP081}

Development and validation of a rapid LC-MS/MS method for simultaneous determination of three TKIS and two tri-azole antifungal agents in human plasma

\author{
Wen Ting Zhang ${ }^{*}$, Yang Zhang', Dong Liu ${ }^{1}$ \\ ${ }^{1}$ Department of Pharmacy, Tongji Hospital of Tongji Medical \\ College, Huazhong University of Science \& Technology, Wuhan, \\ China
}

Background and Objective: Therapeutic drug monitoring (TDM) and appropriate dose-adjustment for both tri-azole antifungals and tyrosine kinase inhibitors (TKIs) are critical for improving treatment success and safety in patients with hematological malignancies. LCMS/MS has been a standard analytical technique for TDM in clinical laboratories. Various methods have been established for simultaneous analysis of antifungal agents or simultaneous determination of TKIs. However, only one class of drug monitoring (tri-azole antifungals or TKIs) is not enough for describing the degree of these DDIs and their clinical influence on patient outcomes. Alternatively, two blood draw may be needed for comprehensive TDM if available. Therefore, an easy, highly sensitive, selective, and rapid LC-MS/MS method that was developed and fully validated for simultaneous determination of three TKIs and two tri-azole antifungal agents in one blood draw.

Method: Plasma samples were collected at the steady-state of drug therapy. Separation is achieved on an Agilent Venusil ASB C18 column using a gradient elution of $10 \mathrm{mM}$ ammonium formate containing $0.2 \%$ formic acid (A) and a mixture of methanol, acetonitrile and isopropanol $(70 / 15 / 15, \mathrm{v} / \mathrm{v} / \mathrm{v})$ containing $0.2 \%$ formic acid at a flow rate of $0.8 \mathrm{~mL} / \mathrm{min}$. A 4000 Triple Quadruple mass spectrometer was operated in positive ionization mode with MRM for highperformance liquid chromatography-tandem mass spectrometry (LCMS/MS) analysis.

Main outcome measures: The robustness of the method with respect to linearity, sensitivity, selectivity, precision, accuracy, matrix effect, extraction recovery, carryover, stability, and hemolysis effect were evaluated based on pre-defined acceptance criteria.

Results: The method was linear over the concentration range of 20.0 to $4000 \mathrm{ng} / \mathrm{mL}$ for imatinib and nilotinib, 50.0 to $10000 \mathrm{ng} / \mathrm{mL}$ for voriconazole and itraconazole, and 2.00 to $400 \mathrm{ng} / \mathrm{mL}$ for dasatinib. It showed satisfactory results in terms of sensitivity, specificity, precision, accuracy, recovery, and stability.

Conclusion: Compared to published LC-MS/MS-based analytical methods, this method merits a high throughput (quick run time of $3.5 \mathrm{~min})$, a small sample volume requirement $(50.0 \mu \mathrm{L})$, a low cost (sample extraction by protein precipitation) and satisfactory lower limits of quantitation. It is especially adaptable to hematological patients undergoing induction, consolidation, stem cell transplantation, and maintenance therapy of TKIs who are concomitantly administered with triazole antifungals.

\section{PP082}

Risk assessment of central nervous system medications use in pregnancy

\author{
Anna Olearova* 1, 2 \\ ${ }^{1}$ Institute of Pharmacology, Faculty of Medicine, Slovak Medical \\ University, ${ }^{2}$ Department of Clinical Pharmacology, University \\ Hospital Bratislava - Ruzinov, Bratislava, Slovakia
}

Background and Objective: Using of pharmacotherapy in pregnancy must be considered very carefully. Due to ethics issues, pregnant women usually don't participate in clinical trials. Safety information on medication use during pregnancy are therefore limited. Risk assessment of the medication use during pregnancy is an important area in clinical pharmacy practice.

Design: The retrospective, one-centre analysis of risk assessments counselling on central nervous system (CNS) medication use in pregnancy was realised. The data were obtained from the Department of Clinical Pharmacology, University Hospital Bratislava, Slovakia. Department provides consultations and risk assessment of drug use during pregnancy. Patients are usually referred at the beginning of pregnancy to assess the risk of drugs used during unknown pregnancy; or before pregnancy to recommend the most safety options. Medical records from counselling settings provided between 2014 and 2020 were included. The age of patient, pregnancy period, specialisation of the referring physician, drugs and indications, recommendations on pharmacotherapy during pregnancy and health status of newborn were recorded. The analysis was focused on CNS drugs used in therapy of epilepsy, schizophrenia, bipolar disorder, depression, anxiety, and sleeping disorders.

Results: General: There were provided 542 consultations on 1435 drugs (2,6 drugs per consultation). The average age of patients was 31,5 years ( $15-44$ years). Consulted drugs were most often used in the $1^{\text {st }}$ trimester $(\mathrm{n}=972)$ and were from the ATC group $\mathrm{N}$ - central nervous system $(\mathrm{n}=418)$ and $\mathrm{J}$ - anti-infectives for systemic use $(\mathrm{n}=362)$. Selected CNS drugs and conditions: There were provided 142 consultations on 246 drugs (1,7 drugs per consultation). Patients were reffered by psychiatrist $(n=64)$ and gynaecologist $(n=54)$ mostly. The most often consulted drugs in treatment of epilepsy were levetiracetam $(n=6)$, lamotrigine $(n=4)$ and valproate $(n=3)$; in treatment of schizophrenia quetiapine $(n=5)$, olanzapine $(n=4)$ and aripiprazole $(n=3)$; in treatment of bipolar disorder quetiapine $(n=5)$ and sertraline $(n=3)$; in treatment of depression escitalopram $(n=11)$, citalopram $(n=9)$; for anxiety alprazolam $(n=15)$; and in treatment of sleeping disorders zolpidem $(n=4)$ and zopiclone $(n=4)$. The most often recommendations were to continue in pharmacotherapy with rigorous prenatal and newborn screening $(n=171)$; to change of therapy was recommended in 21 cases. The health status of newborn was obtain in 8 cases only $(5,6 \%), 7$ children born on time and healthy, one patient underwent abortion.

Conclusion: Analysis showed that it is very important to assess the risk of medication use during pregnancy. It is not necessary to discontinue the medication use during pregnancy in every case. Clinical pharmacist could recommend safety medication options, because the risk of untreated condition could be higher than using of medication. The risk assessment could help physicians to monitor mother and the fetus development, and to focus on possible risk. The role of clinical pharmacist in interdisciplinary approach is very important.

\section{PP083}

Experience with defibrotide to treat hepatic veno-occlusive disease in hematologic patients in a tertiary care teaching hospital

Rubén Gonzalez-García* ${ }^{1}$, Esther Carcelero-San Martin ${ }^{1}$, Gisela Riu-Viladoms ${ }^{1}$, Ines Monge-Escartín ${ }^{1}$, Dolors Soy-Muner ${ }^{1}$

${ }^{1}$ Hospital Clínic de Barcelona, Barcelona, Spain

Background and Objective: Hepatic veno-occlusive disease/sinusoidal obstruction syndrome (VOD/SOS) is a potentially lifethreatening complication during hematopoietic stem cell transplantation. Defibrotide has been approved by EMA for the treatment of serious hepatic VOD/SOS.

Design: We reviewed the experience in a tertiary teaching hospital of patients submitted to hematopoietic stem cell transplantation and 
treated with defibrotide because of confirmed or suspected hepatic VOD/SOS. Clinical courses, chemotherapy and defibrotide electronic prescriptions were retrospectively reviewed in hospital prescription from 2010 until 2019.

Results: During the period reviewed 523 allogeneic stem cell transplantations were done, and 11 of these patients received defibrotide for severe hepatic veno-occlusive disease ( 6 men, 5 women). Causes of allogeneic transplantation: acute myeloid leukemia (AML) $(n=3)$, T-cell lymphoma $(n=2)$, diffuse large B-cell lymphoma $(n=2)$, dendritic cell leukemia $(n=1)$, polycythemia vera $(n=1)$, high-risk myelodysplastic syndrome $(\mathrm{n}=1)$, myelofibrosis $(\mathrm{n}=1)$.

Median age was 52 years (IQR 45-54). All patients had risk factors for VOD: 5 received melphalan, 3 were treated with busulphan and one patient received cyclophosphamide in conditioning regimens; 2 patients were on her second transplantation (one of them had received busulphan on the first one and the second patient had received melphalan); one patient was treated with ifosfamide before transplantation as part of lymphoma treatment. One patient that received melphalan in conditioning regimen had received gemtuzumab for treatment of her refractory AML.

Four patients had received prophylaxis with ursodeoxycholic acid. VOD/SOS symptoms appeared at a median of 9 days after transplantation (range 4-112), hepatic biopsy was performed in 8 patients. Median defibrotide dose received was $25 \mathrm{mg} / \mathrm{kg} / 6 \mathrm{~h} \mathrm{(19-31)}$ and the median days of treatment were 8 (IQR 5-15). At day +100 of transplantation, VOD/SOS was resolved in 4 patients out of $11(36 \%)$ and 5 patients were alive $(45 \%)$. Two patients died as a direct consequence of VOD/SOS.

The most common adverse events (AEs) were hemorrhagic events $(n=2)$, hypotension $(n=2)$ and diarrhea $(n=1)$.

Conclusion: In our cohort, the resolution of the hepatic VOD/SOS at day +100 of transplantation was achieved in $36 \%$ of patients who received defibrotide. The type of the observed adverse events is in line with the previously reported data.

\section{PP084}

Isotretinoin and psychiatric adverse events: a review of the evidence

\section{Thodoris Tsagkaris $^{*}$, MARIOS STAVROPOULOS ${ }^{2}$ \\ ${ }^{1}$ Frederick, FREDERICK UNIVERSITY, NICOSIA, Cyprus, ${ }^{2}$ FREDERICK UNNIVERSITY, PYRGOS HLIAS, Greece}

Background and Objective: Isotretinoin is a widely used therapeutic for the treatment of acne vulgaris and various other skin disorders. However, since its approval, many side effects and contraindications has been described, particularly important, such as teratogenicity as well as liver disease and dermal deterioration. In a very important allegation, isotretinoin has been linked with psychiatric symptoms like depression, suicidal ideation, schizophrenia and hypervitaminosis A syndrome characteristics. These adverse effects have raised significant concerns regarding the safety of isotretinoin. Numerous studies and research have associated isotretinoin with side effects on the mental health of patients and have proposed plausible mechanisms regarding this suspected causative relationship. However, the evidence is still contradicting, and the data disperse making their validity less valuable. Thus, in the present study we aim to analyze further the available literature and present a complete analysis on the side effects of isotretinoin, with particular emphasis on the effects it may have on the mental health of patients.

Design: The review is based on international articles from broad scientific electronic databases like PubMed and Scopus.
Results: This review concludes that although many studies have associated isotretinoin with mental effects like depression, bipolar disorder, schizophrenia, and suicidal ideation the data are still insufficient and often contradictory. In fact, additional studies with accurate data and larger double-blinded samples and more analytic systematic reviews are required. It is especially important to monitor the dose and the intervals that isotretinoin have to be administered in order to potentially cause mental health problems, as well as the duration of treatment and the role that the patient's medical and pharmaceutical history may play.

Conclusion: This review concludes that although many studies have associated isotretinoin with mental effects like depression, bipolar disorder, schizophrenia, and suicidal ideation the data are still insufficient and often contradictory. In fact, additional studies with accurate data and larger double-blinded samples and more analytic systematic reviews are required. It is especially important to monitor the dose and the intervals that isotretinoin have to be administered in order to potentially cause mental health problems, as well as the duration of treatment and the role that the patient's medical and pharmaceutical history may play.

\section{PP086}

Characteristics of drug-drug interactions in patients admitted to the hospital via the emergency department: preliminary findings from a cross-sectional study

Zuzana Očovská* $^{1}$, Martina Maříková ${ }^{1,2}$, Kateřina Kukrálová ${ }^{1}$, Jiří Vlček ${ }^{1,2}$

${ }^{1}$ Department of Social and Clinical Pharmacy, Faculty of Pharmacy in Hradec Králové, Charles University, ${ }^{2}$ Department of Clinical

Pharmacy, Hospital Pharmacy, University Hospital Hradec Králové, Hradec Králové, Czech Republic

Background and Objective: Drug-drug interaction (DDIs) are common in daily practice and could represent a contributory factor of drug-related hospital admissions. The objectives of this poster are a) to characterize potential DDIs identified in the medication history of patients admitted to the hospital via the emergency department (frequency of potential DDIs, Anatomical Therapeutic Chemical classification of medication classes involved in potential DDIs, their mechanism and potential consequences) and b) to characterize manifest DDIs which contributed to the hospitalization of these patients (frequency of manifest DDIs, Anatomical Therapeutic Chemical classification of medication classes involved in manifest DDIs, classification of clinical manifestations according to Medical Dictionary for Regulatory Activities).

Method: The design of this study is cross-sectional. The data for this study were obtained retrospectively from electronic medical records within a broader project focused on identifying drug-related hospital admissions. The medication history of the patients admitted to University Hospital Hradec Králové via the emergency department in August-November 2018 has been screened for the presence of potential DDIs. The identification of potential DDIs has been performed using Micromedex, Lexicomp (via UpToDate) and DrugAgency a.s. database of DDIs. Potential DDI was defined as a DDI with at least moderate severity category in at least one drug interaction screening database. The causality assessment was performed using Drug Interaction Probability Scale (DIPS). Manifest DDI was defined as a DDI with clinical manifestation related to the main or contributory reason of hospital admission with a DIPS score of at least 2 points.

Main outcome measures: Frequency and nature of potential and manifest DDIs. 
Results: A sample of 375 hospital admissions has been analyzed so far. 2273 potential DDIs were identified in 258 hospital admissions and 27 manifest DDIs were identified in 17 hospital admissions. 866 different DDIs were involved in potential DDIs and 22 different DDIs were involved in manifest DDIs which contributed to hospital admission. The most common medication classes involved in potential DDIs included diuretics (16.9\%), antithrombotic agents (13.7\%) and drugs used in diabetes $(10.7 \%)$. Potential DDIs were mostly pharmacodynamic $(79 \%)$ and the most common potential consequences included an increased risk of hypotension (12.4\%), an increased risk of hypoglycemia (11.8\%), an increased risk of bleeding $(9.9 \%)$ and a reduced antihypertensive effect $(7.5 \%)$. Concerning manifest DDIs, the most common clinical manifestation was intestinal hemorrhage and antithrombotic agents (B01A) was the medication class most frequently involved in manifest DDIs.

Conclusion: The frequency and nature of DDIs can provide useful information for clinical pharmacists.

\section{PP087}

Prevalence of pain and rationality of use of analgesics in community-residing and acutely hospitalized seniors in the Czech Republic: results from the EUROAGEISM H2020 ESR7 and the INOMED projects

\section{Adriana Slana ${ }^{*}{ }^{1}$, Adriana Magátová ${ }^{1}$, Gabriela Vaculová ${ }^{1}$, Jindra Reissigová ${ }^{1,2}$, Jovana Brkic ${ }^{1}$, Daniela Fialová ${ }^{1,3}$}

${ }^{1}$ Department of Social and Clinical Pharmacy, Faculty of Pharmacy in Hradec Králové, Charles University, Hradec Králové, ${ }^{2}$ Department of Statistical Modelling, Academy of Science, ${ }^{3}$ Department of Geriatrics and Gerontology, 1st Faculty of Medicine, Charles University, Prague, Czech Republic

Background and Objective: The proper diagnosis of pain and its appropriate management are crucial, particularly in older patients (in order toprevent somatic and neuropsychoatric complications, chronification of pain and worsening of some commorbidities, eg. geriatric dementia and depression). The aim of our study was to identify and compare the prevalence of pain, its main characteristics andthe rationality of analgesic drug use in community-residing and acutely hospitalized older patients in the Czech Republic.

Method: Data of 1159 Czech seniors $65+(\mathrm{N}=589$ acute care, $\mathrm{N}=$ 563 ambulatory care, selected from 4 cities of 4 different regions) were prospectively collected in the EuroAgeism H2020 ESR7 international project in 2018-2019 using protocols of comprehensive geriatric assessment (including over 300 socio-demographic, clinical, functional and medication-related geriatric characteristics). Descriptive statistics was applied using R-software (version 4.0.3).

Main outcome measures: The prevalence of pain (including asessment of all comprehensive pain characteristics, as well as pain intensity before and after medication treatment using VAS- visual analogue scale), appropriateness of selection of analgesics (with a special focus on opioids and weak opiods) and comparisons of rationality of prescribing between acute and ambulatory care in the Czech Republic.

Results: In the total sample, more than half of seniors $(58.1 \%)$ suffered from pain $(41.7 \%$ seniors reported chronic pain, $18.9 \%$ acute pain and $4.7 \%$ breakthrough pain). The highest prevalence was documented for pain experienced 2-3 times a week (22.3\%) and several times a day (21.2\%). $10.2 \%$ patients in acute care and $5.0 \%$ in ambulatory care took opiods $(\mathrm{p}<0.001)$. Of those, half $(50 \%)$ were treated by weak opioids, $5 \%$ by combination of weak opioids with other analgesics, $3.2 \%$ by strong opioids and $1.3 \%$ by combinations of more opioids. During assessment of pain intensity reduction (according to VAS) strong opioids were the most efficient in ambulatory care (in average reduction of VAS from 8 to 2) whereas weak opioids combined with NSAIDs were the most used and efficient in acute care (reduction VAS from 6 to 1 ).

Conclusion: Our pilot findings confirmed significantly higher prevalence of opioids prescription in acute than ambulotory care and overal high prescription of weak opioids (in over $50 \%$ of patients treated by analgesics). In terms of efficacy of the analgesics, strong opioids had the higher efficacy in reduction of pain intensity in ambulatory practices, while weak opioids combined with NSAIDs were more prescribed and enough effective in acute care.

Grants: EuroAgeism Horizon 2020 MSCF-ITN-764632, Inomed NO.CZ.02.1.01/0.0/0.0/18_069/0010046, Progress Q42- Faculty of Pharmacy, Charles University (KSKF- group 2), START/MED/093 CZ.02.2.69/0.0/0.0/19_073/0016935, SVV260 551 and I-CARE4OLD H2020 -965341

\section{PP088}

Potentially inappropriate medication use identified using U.SForta (fit for the aged) list in older patients attending chronic care clinics in Ethiopia

\section{Akshaya S. Bhagavathula* 1 , Mohammed A. Seid ${ }^{2}$, Aynishet Adane $^{3}$, Eyob A. Gabreyohannes ${ }^{4}$, Daniela Fialova ${ }^{1,5}$}

${ }^{1}$ Social and Clinical Pharmacy, Faculty of Pharmacy at Hradec Kralove, Charles University, Hradec Kralove, Czech Republic, ${ }^{2}$ Clinical Pharmacy, School of Pharmacy, College of Medicine and Health Sciences, University of Gondar, ${ }^{3}$ Internal medicine, School of Medicine, College of Medicine and Health Sciences, University of Gondar, Gondar, Ethiopia, ${ }^{4}$ Division of Pharmacy, School of Allied Health - University of Western Australia, Perth, Australia, ${ }^{5}$ Geriatrics and Gerontology, 1st Faculty of Medicine, Charles University, Prague, Czech Republic

Background and Objective: Potentially inappropriate medication (PIM) use represents a significant problem in older patients. Therefore, identifying PIMs and improving the medication scheme expert consensus (ex: FORTA list) could be potentially valuable. To determine the extent of PIM use in older outpatients using the U.S.FORTA (Fit fOR The Aged) List in Ethiopia.

Design: A cross-sectional study was conducted in older patients attending chronic care clinics at a tertiary care teaching hospital from March to August 2020. A comprehensive medication review was performed, and identified medications were labeled according to the U.S FORTA List, namely A (indispensable), B (beneficial), C (questionable), to D (to avoid) after investigating the age-appropriateness of medication use.

Results: A total of 320 older patients $(65+$ years $)$ were included in the study. The mean age of the study population was $71.9 \pm 6.0$ years. Overall, $59.1 \%$ of the older patients had at least two chronic comorbidities, and the mean number of medications prescribed per patient was $3.4 \pm 1.7$. A total of 190 medications listed in the U.S FORTA were identified, and the extent of PIM use (at least one PIM according to the U.S FORTA list) was $24.7 \%$. The identified medications were labeled A (61.5\%), B (12.1\%), C (11\%), and D (15.2\%), according to the U.S FORTA list. Among administered drugs to avoid use in older adults were amitriptyline $(9.2 \%)$, glimepiride $(4.7 \%)$, and phenytoin $(1.3 \%)$. Drugs to use with caution included spironolactone in hypertensive patients $(8.5 \%)$ and verapamil in patients with atrial fibrillation $(2.5 \%)$.

Conclusion: This is the first study to investigate the appropriateness of medication use using the U.S FORTA list in Ethiopia that confirmed a quarter $(24.7 \%)$ of medications were prescribed 
inappropriately to older patients. This study underlines the importance of awareness, education, and preventive interventions to increase the rationality of geriatric prescribing in Ethiopia.

Acknowledgement: EuroAgeism H2020 MSCF-ITN-764632, Inomed NO.CZ.02.1.01/0.0/0.0/18_069/0010046, Progress Q42Faculty of Pharmacy, Charles University, START/MED/ 093CZ.02.2.69/0.0/0.0/19_073/0016935, SVV 260551 and ICARE4OLD H2020 -965341.

\section{PP089}

\section{Assessment of the use of intravenous albumin in a tertiary} hospital

\section{Bárbara Cancela Díez ${ }^{*}$, Felix Gómez-De Rueda ${ }^{2}$, Nuria Martinez-Casanova $^{1}$, Isabel Moya-Carmona ${ }^{1}$}

${ }^{1}$ Pharmacy, Hospital Virgen de la Victoria, Málaga, ${ }^{2}$ Pharmacy, Hospital Virgen Macarena, Sevilla, Spain

Background and Objective: Human albumin is a physiological plasma-expander, but its use is controversial because there is not enough clinical evidence in some (clinical) situations. Furthermore, its limited availability make it essential to define recommendations for its appropriate use.

To evaluate the indications and use of intravenous albumin in a tertiary hospital and to estimate the related cost of inappropriate use of albumin.

Design: A prospective observational study was carried out between May and July 2019. Information of all patients treated with albumin in all units of the hospital except the Intensive Care Unit was collected. The variable chosen were: Age, sex, diagnosis, medical indication of the use of albumin, prescriber service, treatment duration and levels of total protein, albumin and prealbumin. To evaluate de appropriate indications of use albumin we took as reference the Andalusian health service (AHS) common agreement of use albumin. Results: 55 patients were treated with albumin (33 males, 22 females), average age was $66,0+-11.59 .47,27 \%$ was prescribed by digestive unit, $18,18 \%$ by internal medicine unit, $9,09 \%$ by respiratory medicine unit, $7,27 \%$ by onco-hematology unit, urology, cardiology and digestive surgery unit: $3,63 \%$ each one. The units with less albumin prescriptions were traumatology, vascular surgery and gynecology $(1,81 \%$ each one). Average duration of treatment was $7,5 \pm 7,7$ days. Analysis revealed a mean total protein (unknow in 18 patients) of $6,0 \mathrm{~g} / \mathrm{dl}$, mean albumin $2,3 \mathrm{~g} / \mathrm{dl}$ (unknow in 5 patient). Prealbumin levels were lower than $2,4 \mathrm{mg} / \mathrm{dl}$ in 11 patients $(20 \%)$ (levels were not available in 27 patients). The main reason for albumin use was ascites non responsive to diuretics (29.0\%), followed by ascites/paracentesis (25,4\%), edema responsive to diuretics $(14,5 \%)$, spontaneous bacterial peritonitis $(9.0 \%)$, nephrotic syndrome $(5,4 \%)$, non-haemorrhagic shock $(5,4 \%)$, hypoalbuminaemia in the absence of oedema and acute hypotension $(3,6 \%)$, haemorrhagic shock $(1,8 \%)$ and hepatorenal syndrome $(1,8 \%)$. The albumin indication was not available in 2 patients. The use of albumin was appropriate in $69 \%$. In 17 patients the use of albumin was not indicated according to the common agreement of use albumin of AHS and the total cost of albumin in these patients amounts to $11.110,6 €$.

Conclusion: Even though the use of albumin is mainly as recommended, in some clinical situations its use can be controversial. In these situations to revise routinely its application instructions could prevent the complications associated to its use, according to bibliography, and can also make the cost cheaper but further studies are necessary to confirm these facts.

\section{PP090}

Dexamethasone positioning in therapeutic management of COVID-19

Cécilia N'Guessan ${ }^{*}{ }^{1}$, Raul Diaz Salmeron ${ }^{1}$, Jean-Jacques Houri ${ }^{1}$, Valentin Pouilleau ${ }^{1}$, Claude Bernard ${ }^{1}$, Marie-Pierre Berleur ${ }^{1}$

${ }^{1}$ AGEPS, AP-HP, Paris, France

Background and Objective: In the midst of the global health COVID-19 crisis, a new hospital preparation: DEXAMETHASONE AP-HP $6 \mathrm{mg}$ (vial of 50 tablets) was made available thanks to the «Etablissement Pharmaceutique » of « Assistance Publique-Hôpitaux de Paris » (EP of AP-HP). Therapeutic use declared to French Health Authority (ANSM) is treatment of coronavirus SARS-CoV-2 (COVID-19) infection in adults and adolescent over age 12 or weighing more than $40 \mathrm{~kg}$, who need oxygen therapy. This new hospital preparation is a more convenient pharmaceutical form at the dose of 1 tablet per day versus the current drug on the French market which requires more than ten tablets per day. Objective of our work: Positioning DEXAMETHASONE AP-HP $6 \mathrm{mg}$ in therapeutic management of COVID-19.

Design:

- Analysis of RECOVERY clinical trial.

- Recommandations of expert committee.

- Analysis of internal reports: 'Evaluation de l'aptitude de performance du procédé de la préparation hospitalière DEXAMETHASONE AP-HP 6 mg, comprimé' and 'Evaluation de la cinétique de dissolution in vitro de la préparation hospitalière DEXAMETHASONE AP-HP $6 \mathrm{mg}$, comprimé'.

- Preparation of the information materials and communication strategy.

Results: This hospital preparation was made available in COVID-19 background thanks to two key factors (i) clinically, results of RECOVERY clinical trial about dexamethasone use demonstrated a benefit on mortality in patients with oxygen therapy $(\geq 3 \mathrm{~L} / \mathrm{min})$ and allowed to add dexamethasone in the pipeline. This clinical trial compared dexamethasone (6 mg per day orally for 10 days) versus standard care in population of 6425 hospitalized patients with a SARS-CoV-2 infection. Mortality rate on the 28th day has been statistically reduced in dexamethasone group in comparison with control group. (ii) From the pharmaceutical perspective, EP of AP-HP has expertise on dexamethasone tablets manufacturing. This new hospital preparation has a dissolution profile which complies with European pharmacopeia requirements and an effective manufacturing process. The packaging of this hospital preparation, which is not optimal (blister would be better) is now being discussed. This hospital preparation offers a 20 days shelf life after the vial first opening. The communication plan included a letter, an information note and a Question \& Answer model, which has enabled to answer to hospital pharmacists.

Conclusion: This new hospital preparation has been distributed over the whole French territory, and hospitals of different sizes and locations have been interested in its use. In the long run, this hospital 
preparation could be envisaged in other serious viral diseases responsible for respiratory distress syndrome.

\section{PP091}

Rantes, leptin, IL-1B and TNF- $\alpha$ evolution in refractory epileptic patients

\author{
Daniela Farias $^{*}{ }^{1}$, Filipa Gouveia ${ }^{1}$, Andreia Carona ${ }^{1}$, Joana \\ Bicker $^{1,2}$, Francisco Sales ${ }^{3}$, Amílcar Falcão ${ }^{1,2}$, Ana Fortuna ${ }^{1,}$ \\ ${ }^{1}$ FACULTY OF PHARMACY OF UNIVERSITY OF COIMBRA, \\ ${ }^{2}$ CIBIT - Coimbra Institute for Biomedical Imaging and Tanslational \\ Research, University of Coimbra, ${ }^{3}$ Refractory Epilepsy Reference \\ Centre, Centro Hospitalar e Universitário de Coimbra, Coimbra, \\ Portugal
}

Background and Objective: Numerous antiepileptic drugs (AEDs) are currently available in clinical practice, however approximately $1 / 3$ of the patients remain untreated. One of the underlying causes that has been hypothesized is the neuroinflammatory state of epileptogenesis, characterized by high-production of proinflammatory cytokines.

This study aimed at correlating the serum levels of RANTES, leptin, Interleukin (IL)- 1 and Tissue Necrosis Factor- $\alpha$ (TNF- $\alpha$ ) in individuals with refractory epilepsy that are being treated with levetiracetam or/and other(s) antiepileptic(s).

Method: The study included 48 patients with refractory epilepsy that were followed at the Refractory Epilepsy Centre of Centro Hospitalar e Universitário de Coimbra between December 2019 and October 2020. Four of them were excluded once they were admitted to the Intensive Care Unit. RANTES $(n=40)$, leptin $(n=44)$, IL-1 $\beta(n=44)$ and TNF- $\alpha(n=36)$ levels were determined by ELISA, resorting to manufacturer protocols. Four groups were created: patients treated with levetiracetam (Group 1), levetiracetam plus one AED (Group 2), levetiracetam plus two AEDs (Group 3) and other AED without levetiracetam (Group 4). Results were compared with the Control group that included 18 healthy subjects. Age, gender, body mass index, daily dose and plasma concentrations of levetiracetam were collected and compiled. All statistical tests were performed in GraphPad Prism ${ }^{\circledR} 7$ software.

Main outcome measures: Plasma concentrations of the four studied cytokines were compared between the four test groups of study and with the control group.

Results: Comparatively with control group, group 1 revealed higher values of leptin $(\mathrm{p}=0.0004)$ but lower values of TNF- $(\mathrm{p}=0.0208)$. Group 2 also presented higher RANTES $(p=0.0462)$ and leptin concentrations $(\mathrm{p}=0.0069)$, while groups 3 and 4 exhibited no differences with control group. IL-1 remained comparable in all groups even though with a tendency to decreased values, when compared to control.

Conclusion: The pro-inflammatory cytokines RANTES and leptin are enhanced in treated refractory epileptic patients, suggesting that they can be biomarkers of this pathology. In opposition, TNF- $\alpha$ decreased, particularly in levetiracetam-treated patients, corroborating levetiracetam anti-inflammatory effect.

Acknowledges: This work was financed by FEDER funds through Portugal 2020 in the scope of the Operational Programme for Competitiveness and Internationalization, and Fundação para a Ciência e Tecnologia (FCT), Portuguese Agency for Scientific Research, within the scope of the research project POCI-01-0145-FEDER-030478
PP092

Efficacy and safety of single-agent panitumumab in unresectable cutaneous squamous cell carcinoma: a retrospective analysis

Gwladys Claustre ${ }^{*}{ }^{1}$, Coralie Boulanger1, Chloé Gossery ${ }^{1}$, Antonin Maréchal ${ }^{1}$, Florian Slimano ${ }^{1}$

${ }^{1}$ Pharmacy, Reims University Hospital, Reims, France

Background and Objective: Cutaneous Squamous Cell Carcinoma (cSCC) is associated with population aging and poor prognosis. Cetuximab, an EGFRi (Epidermal Growth Factors Receptor inhibitor) is used in unresectable cSCC, alone or combined with platin/fluorouracil. Panitumumab, another EGFRi, is commonly used in our center based on one study ${ }^{1}$. The aim of this study was to describe the efficacy and the safety of single-agent panitumumab in the treatment of patients with unresectable cSCC.

Design: This retrospective study included the data from all patients who received panitumumab in one University Hospital for the treatment of unresectable cSCC from January 1st, 2016 to July 31th, 2019. Demographic, clinical and biological data were extracted from electronic health records. Overall Survival (OS) and Progression-Free Survival (PFS) were estimate according to the Kaplan-Meier method. Results: $\mathrm{N}=29$ patients were included (median age $83 \pm 11$ years; sex ratio male/female of 2.2$)$. The majority $(76 \%, n=22)$ of patients had loco-regionally advanced disease without metastasis and was treatment naive $(48 \%, \mathrm{n}=14)$. At the end of the follow-up, 10 patients (34\%) were alive. The median OS and PFS were 10.0 and 5.0 months, respectively. Panitumumab was mainly discontinued because of progression $(45 \%, n=13)$, toxicity $(28 \%, n=8)$ and death $(17 \%, n=5)$. Ninety-seven percent of the patients $(n=28)$ experienced adverse effects (none of grade 4). Most of them were cutaneous toxicity (76\%, $\mathrm{n}=22)$, fatigue $(59 \%, \mathrm{n}=17)$ and ocular toxicity $(34 \%, \mathrm{n}=10)$.

Conclusion: The literature reports one study with median OS and PFS of 11.0 and 8.0 months, respectively ${ }^{1}$. The younger median age (68 years in the study) could explain the difference with our results. Panitumumab appears as a safe alternative to cetuximab for the treatment of patients with unresectable cSCC. Further direct comparative studies are needed to assess the alternative in terms of efficacy and tolerance. Economic impact should be also considered and must take into account the advent of biosimilars.

${ }^{1}$ Foote MC, et al. Phase II study of single-agent panitunumab in patients with incurable cutaneous squamous cell carcinoma. Ann Oncol 2014; 25: 2047-2052

\section{PP093}

Application of criteria screening tool of older person's potentially inappropriate prescriptions as a tool for detecting inappropriate prescription in a nursing home

Javier Garcia Marin* ${ }^{1}$, Marina Fages Perez ${ }^{1}$, Javier Romero Puerto $^{2}$, Sol P. Cortes de Miguel ${ }^{1}$, Alberto Soria Martin ${ }^{1}$

${ }^{1}$ FARMACIA, HOSPITAL PUNTA DE EUROPA, ALGECIRAS, ${ }^{2}$ HOSPITAL NAVALMORAL DE LA MATA, CACERES, Spain

Background and Objective: A series of tools have been developed that allow us to detect inappropriate prescriptions in elderly polymedicated patients. It stands out a group of criteria for the detection of inappropriate medication in these population, the STOPP/START 
criteria (Screening Tool of Older Person's potentially inappropriate Prescriptions/Screening Tool to Alert doctors to the Right, i.e. appropriate, indicated Treatment)

The objectiv is to detect inappropriate prescription of medications in pacients of a nursing home by applying the STOPP criteria

Design: The inclusion criteria were patients with age over 65 years and more than 5 prescription drugs. The STOPP criteria selected were those applied to the patients to detect inappropriate prescriptions. ATHOS-Prisma ${ }^{\circledR}$ was used to identify prescription drugs in each patient, while medical records and analytical were reviewed by Diraya.

Results: 38 patients were evaluated obtaining a total of 48 inappropriate prescriptions: related to the central nervous system (28), 22 were due to the use of benzodiazepines for more than four weeks; the concomitant use of antimuscarinic drugs (5); the concomitant prescription of two drugs of the same class (3); the prescription of benzodiazepines in patients with respiratory failure (3); loop diuretics as first-line treatment of hypertension or in patients with urinary incontinence (3); use of acetylsalicylic acid in chronic treatment at dose higher than $160 \mathrm{mg} /$ day (2); use of non-steroidal anti-inflammatory drugs (NSAIDs) in patients with severe hypertension (2); and with the use of opiates without concurrent use of laxatives (2)

Conclusion: The STOPP criteria allow to detect inappropriate prescription in order to reduce pharmacological interactions and adverse reactions in polymedicated and pluripathological patients. Some criteria have not been applied because of the lack of recents blood tests. Therefore, it would be necessary monitoring the nursing home patients with more frequency

\section{PP094}

Descriptive study of the COVID19 adult patient and its pharmacological treatment in a hospital of specialties

\section{Marina Fages Perez ${ }^{1}$, Javier Garcia Marin* ${ }^{*}$, Alberto Soria Martin $^{1}$, Javier Romero Puerto ${ }^{2}$, Adriana Moreno Herrera ${ }^{1}$ \\ ${ }^{1}$ FARMACIA, HOSPITAL PUNTA DE EUROPA, ALGECIRAS, ${ }^{2}$ HOSPITAL NAVALMORAL DE LA MATA, CACERES, Spain}

Background and Objective: To characterize the profile of the patient admitted for COVID-19 in a specialty hospital.

Design: Descriptive study of patients who required hospital admission with positive PCR confirmation for COVID-19 during the period March-June 2020, both inclusive. The following variables were collected: age, sex, number of days of hospital admission, number of days from the onset of symptoms to initiation of treatment, days and type of treatment, type and number of comorbidities, and death (yes / no). The data were obtained from the Unique Digital History (Diraya $\left.{ }^{\circledR}\right)$. Statistical analysis was performed using the SPSS v.15 program.

Results: A total of 79 patients were included. The distribution by age and sex was: 55 men $(69.5 \%)$ and 24 women $(30.4 \%)$, with a mean age of 68 years, being $57 \%$ older than 65 years. The mean days of hospital admission was 16 (1-68) and days from the onset of symptoms to the initiation of treatment was $6.5( \pm 4.8)$, while the mean duration of treatment was 9.5 days. 59 patients $(75 \%)$ presented one or more comorbidity, the most frequent being arterial hypertension $(48.1 \%)$, diabetes $(30.4 \%)$, dyslipidemia $(20.3 \%)$, ischemic heart disease (20.3\%), active neoplasia (10.3\%) and COPD (10.1\%). 24 patients died (15 men and 9 women, 21> 65 years and 22 with some associated comorbidity). Regarding the type of treatment, all patients received lopinavir / ritonavir plus hydroxychloroquine, except for 4 patients who received hydroxychloroquine + interferon beta, 2 patients who received hydroxychloroquine + azithromycin, 1 patient who received remdesevir as monotherapy, and 2 cases that were asymptomatic they did not require treatment. 20\% (16 patients), in addition to receiving any of the previous treatments, received a single dose of 400-600 mg depending on the weight of intravenous tocilizumab, with the exception of two patients who required a second dose. Of the 16 patients who received tocilizumab, 8 died. Finally, only one patient received ruxolitinib at a dose of $5 \mathrm{mg} / 12 \mathrm{~h}$, who finally died.

Conclusion: The profile of the patient admitted for COVID-19 in our setting is male, over 65 years of age, with one or more comorbidities, the most frequent being hypertension and diabetes. Regarding treatment, the combination lopinavir/ritonavir plus hydroxychloroquine with or without azithromycin was the most used

\section{PP095}

Alert prescribing of clozapine: a comparison of five drug drug interaction sources

Jeroen Govaerts $^{*}{ }^{1}$, Annelies Verluyten ${ }^{2}$, Filip Bouckaert ${ }^{3}$, Marc De Hert ${ }^{3}$, Franciska Desplenter ${ }^{2}$

${ }^{1}$ UPC KULeuven, Leuven, Belgium, ${ }^{2}$ Department of Pharmaceutical and Pharmacological Sciences, ${ }^{3}$ Department of Neurosciences, UPC KULeuven, Leuven, Belgium

Background and Objective: Clozapine, an antipsychotic used in the management of treatment resistant schizophrenia, is known for its serious side effects. In order to promote patient safety, drug drug interactions should be taken into account when prescribing clozapine. Drug drug interaction databases are often used by clinicians or incorporated in hospital software. It is hypothesized that discrepancies exist in risk taxation between different sources on drug drug interactions. Thus relying on a single source could result in over- or underestimation of the interaction risk. In this study five sources on drug drug interactions with clozapine were examined in order to determine the degree of consensus on clozapine interaction severity. Furthermore an evaluation on the severity was made for each included potential drug drug interaction.

Method: The summary of product characteristics of clozapine, Delphicare interaction database, Stockley's interaction checker, the Lexicomp interaction database and the interaction database of Clinical Pharmacology were included. The original categories of each source on the included interactions were divided into three categories; contraindicated, caution needed and safe. By comparing the assigned catergories, consensus between sources was determined. Furthermore, based on the combined information from all included sources, an evaluation on the severity of each potential interaction was made.

Main outcome measures: The degree of consensus between the included sources on all potential drug drug interactions with clozapine.

Results: 183 potential drug drug interactions with clozapine are retrieved from the five included sources. A consensus between sources is found in $47.5 \%(n=87)$ of drug drug interactions, with only $27.3 \%(\mathrm{n}=50)$ of included DDI's reported by all sources. After evaluation of all databases, $11.5 \%(n=21)$ of all included DDIs are evaluated as 'contraindicated' and $88.0 \% \quad(n=161)$ as 'caution needed'.

Conclusion: This study shows major discrepancies between five different sources on drug drug interactions with clozapine. It is hypothesized that reasons for these discrepancies lie in the lack of solid evidence on DDI with clozapine. The potential impact of the use of one specific database on patient safety and prescribing behavior could prove to be problematic. Although primary literature on DDIs with clozapine is much needed, our comparison of different sources 
on DDI may help clinicians in evaluating the safety of specific drug combinations and managing further monitoring of a potential DDI with clozapine.

\section{PP096}

Pharmacogenetic interventions to improve outcomes in patients with multimorbidity or prescribed polypharmacy: a systematic review

\section{Joseph O'Shea* 1 , Mark Ledwidge ${ }^{2}$, Joseph Gallagher ${ }^{2}$, Catherine Keenan ${ }^{1}$, Cristín Ryan ${ }^{1}$ \\ ${ }^{1}$ Trinity College Dublin, ${ }^{2}$ University College Dublin, Dublin, Ireland}

Background and Objective: The delivery of healthcare is primarily built around singular diseases, yet ageing populations and increased prevalence of chronic diseases globally means greater combined burden of multimorbidity and polypharmacy. Traditional interventions are not widely effective; a more holistic and integrated approach to healthcare delivery is required for these patients. Pharmacogenetic or pharmacogenomic analysis has potential as a component of medicines optimisation. However, the adoption of pharmacogenetics in this respect may require more evidence. Our objective was to investigate the effect of pharmacogenetic interventions on outcomes in adults with multimorbidity or prescribed polypharmacy in all healthcare settings.

Method: PubMed, Embase, CENTRAL, CINAHL, AMED, PsycInfo and several clinical trials registers were searched for studies involving multi-medicine pharmacogenetics in adults with multimorbidity or polypharmacy. Risk of bias assessment was performed per Cochrane guidelines. Narrative synthesis was undertaken to summarise the data; meta-analysis was inappropriate due to the heterogeneity of included studies.

Main outcome measures: Studies were included without restrictions on methodological design if they reported on at least two outcomes derived from consensus-based core outcome sets for multimorbidity and polypharmacy.

Results: The search yielded 10,725 citations, of which fifteen studies of diverse design and variable quality met the inclusion criteria. Six non-comparative studies, three observational studies, three randomised controlled studies, and three ongoing studies in primary care, mostly involving pharmacist-led medication management, were included. The studies reported effects on health service utilisation, estimated improvements in healthcare costs, enhanced drug interaction identification, and reinforced clinical decision-making. One small randomised study demonstrated encouraging impacts of pharmacogenetics on hospitalisation rates using a multi-gene, multi-drug, multi-disease, pharmacist-led medicines optimisation intervention.

Conclusion: The incorporation of pharmacogenetic screening into medication optimisation for adults with multimorbidity and polypharmacy could have significant benefits for patients and health systems. However, due to study design heterogeneity and the quality of the included studies, it is difficult to draw generalisable conclusions. Further pragmatic, robust studies looking at pharmacogenetics in diverse, real-world patient populations, are required to establish the benefit of multi-medicine pharmacogenetic screening on patient outcomes.

\section{PP097}

Analysis of effectiveness of ceftolozane/tazobactam in the treatment of infections by multiresistant microorganisms in a third-level hospital

\section{Margarita Beltrán-García* ${ }^{1}$, Marta Valera-Rubio ${ }^{1}$, Marisa Moya-Martinez ${ }^{1}$, Santiago Sandoval-Fernández del Castillo ${ }^{1}$, Miguel Ángel Calleja-Hernández ${ }^{1}$ \\ ${ }^{1}$ FARMACIA, HOSPITAL UNIVERSITARIO VIRGEN MACARENA, SEVILLA, Spain}

Background and Objective: Ceftolozane/Tazobactam $(\mathrm{C} / \mathrm{T})$ is a new combination of 2 nd generation cephalosporin with a beta-lactamase inhibitor. It is especially useful for multi-resistant Pseudomonas aeruginosa due to its stability in the presence of AmpC-type betalactamases and because it is not affected by loss of external membrane porins $(\mathrm{OprD})$ or by the presence of active expulsion pumps. Our objective was to analyze the pattern of utilization and efficacy of $\mathrm{C} / \mathrm{T}$ in clinical practice from its commercialization to the present in a third-level hospital.

Design: Retrospective, single-center and descriptive study of 5 years duration (May 2016-May 2021), which included all patients who received antibiotic treatment with $\mathrm{C} / \mathrm{T}$.

The following data was collected; demographic variables -sex and age-, clinical variables: type of infection, indication -empirical or directed-, isolated microorganism, duration of treatment, dose administered, clinical unit and antibiotics tested in the antibiogram. Effectiveness was assessed by clinical and microbiological improvement after 30 days of treatment, specifically the crude mortality rate and the readmission rate at 30 days.

Results: 23 patients were selected, 15 of them were men (65\%), with a mean age of $61.7 \pm 12$ years. The clinical units involved were: ICU (8), infectious diseases unit (6), General Surgery (4), Internal Medicine (4) and Hematology (1). Treatment was directed in 16 cases (70\%). The indications for empirical treatment were 4 nosocomial pneumonia and 3 skin and soft tissue infection (SSTIs), with a median duration of 4 days (2-21). In the cases of directed treatment there were: 8 pneumonias -4 of them associated with mechanical ventilation, 2 nosocomial and 2 bronchiectasis-, 6 SSTIs, 1 intra-abdominal infection and 1 bacteremia, with a median duration of 14 days (3-38). Multiresistant Pseudomonas aeruginosa was isolated in all cases and only sensitive to aminoglycosides and Colistin, in addition to $\mathrm{C} / \mathrm{T}$. Resolution of the infection occurred in 12 patients with targeted therapy (75\%) and in 3 patients with empirical therapy (43\%). In the rest it was death: directed 4 and empirical 4 . Only 2 patients had continuous readmissions due to COPD gold IV with bronchiectasis and colonization by Pseudomonas aeruginosa.

Conclusion: $\mathrm{C} / \mathrm{T}$ has been used in accordance with the indications referred to by the Therapeutic Positioning Report of the Ministry of Health of our country, which advised its use in patients with a high probability of isolation of Pseudomonas aeruginosa, focusing mainly on the most severe cases complicated by patient comorbidity.

\section{PP098}

Ruxolitinib in corticosteroid refractory graft versus host disease: experience in our center

Maria Margalida Mestre Ribot ${ }^{*}{ }^{1}$, Inés Monge ${ }^{1}$, Gisela Riu ${ }^{1}$, Esther Carcelero ${ }^{1}$, Marta Prat ${ }^{1}$, C Martínez ${ }^{1}$, M Suárez-Lledó ${ }^{1}$, Dolors Soy ${ }^{1}$

${ }^{1}$ Hospital Clínic de Barcelona, Barcelona, Spain 
Background and Objective: Graft versus host disease (GVHD) is a major complication after allogeneic stem cell transplantation. Despite second line treatment is not yet well established, ruxolitinib seems to be a promising option in view of results of a clinical trial and several case reports.

The aim of this study is to describe the experience with ruxolitinib in patients with acute or chronic refractory GVHD treated in a tertiary care teaching hospital.

Design: Clinical courses, biochemistries and dispensations of ruxolitinib were retrospectively reviewed. Hematologists specialized on stem cell transplantation graded the severity of GVHD and evaluated the response according to National Institute of Health 2014 criteria. Adverse effects were classified following National Cancer Institute Common Terminology Criteria.

Results: Twelve patients received ruxolitinib (median age 52 years [range 19-66]). Six patients had a sibling donor and other six had a HLA-matched unrelated donor. Eight received a myeloablative conditioning regimen, whether four received intensity reduced ones. In all cases, stem cell source was peripheral blood.

Four patients received ruxolitinib for acute GVHD (aGVHD). Median onset was 30.5 days [14-54]. They had received between three and four lines of previous treatment. Three of these patients did not show response and died due to complications derived from GVHD. One patient with cutaneous aGVHD grade III responded and was 211 days on treatment.

Eight patients received ruxolitinib for cGVHD. The median onset was 290 days [140-978]. They had received between one and four lines of previous treatment. By now, median time of ruxolitinib treatment is 19.4 months [3.3-38.9]. Five patients are still ongoing treatment. Three patients did not respond, three showed a partial response and two had a complete response.

Conclusion: Ruxolitinib was useful in one case of aGVHD out of four, and in five patients with cGVHD out of eight. Our experience shows less response in aGVHD compared to the recently published clinical trial, where ruxolitinib was used as preferred second line treatment. Our results in cGVHD are in line with previous experience published.

\section{PP099}

Theoretical exploration of development and implementation of antimicrobial stewardship programs in hospitals in the United Arab Emirates: a qualitative study of the perspectives of key stakeholders and health professionals

\section{Nortan Hashad* 1, 2 , Derek Stewart ${ }^{3}$, Dhayaneethie Perumal ${ }^{4}$,} Najiba Abdul Razzaq ${ }^{5}$, Antonella Pia Tonna ${ }^{1}$

${ }^{1}$ School of pharmacy and life sciences, Robert Gordon University, Aberdeen, United Kingdom, ${ }^{2}$ Pharmacy, Higher colleges of technology, Dubai, United Arab Emirates, ${ }^{3}$ College of Pharmacy, Qatar University, Doha, Qatar, ${ }^{4}$ Commission of Academic Accreditation, Ministry of Education, Abu Dhabi, ${ }^{5}$ Ministry of health and prevention, Dubai, United Arab Emirates

Background and Objective: Antimicrobial Resistance (AMR) has been declared as a public health emergency and has led to the establishment of Antimicrobial Stewardship Programs (ASP) to enhance prudent use of antimicrobials ${ }^{1}$. In the United Arab Emirates (UAE), ASP has been mandated by some health authorities; yet few studies report ASP implementation in UAE.

The aim of this study is to explore ASP development and implementation in hospitals in UAE and to enable characterization of key facilitators, barriers and solutions at the level of both healthcare providers and key stakeholders.
Method: A phenomenological qualitative approach has been adopted using semi-structured interviews with three groups of participants; Local health authority representatives, ASP members and practitioners in hospitals. An interview schedule was developed and piloted based on available literature and grounded in the Consolidated Framework for Implementation Research (CFIR). Participants were recruited via purposeful and snowball sampling. Interviews were conducted, audio recorded, transcribed and independently analyzed by two researchers based on CFIR framework ${ }^{2}$.

Main outcome measures: Outcomes included perspective and views of participants from different groups in relation to ASP development and implementation in UAE. Views will be compared to provide recommendations for optimising ASP implementation and development.

Results: Thirty-five interviews (approximately 45-60 min) were conducted with authority representatives $(n=4)$, ASP members $(n=$ 23) and hospital practitioners $(n=8)$ with data saturation achieved. ASP development in UAE was driven by various internal and external factors such as international accreditation and AMR data. ASP structure was adapted from international guidelines and influenced by local hospitals with experience in ASP implementation. Adaptations varied depending on local available resources. Facilitators included; leadership support, availability of continuous education and effective communication across the hospital. Barriers included; lack of financial support, lack of human resource mainly infectious disease physicians, clinical pharmacists and microbiologists, and inadequate information technology. The Covid-19 pandemic was considered a significant set-back for ASP activities.

Conclusion: ASP implementation in UAE is progressing with potential for more streamlined and future expansion. There is a need to secure financial support, leadership commitment and human resources to accelerate the implementation process.

References: (1) World Health Organization. Antimicrobial Resistance Global Report on Surveillance. Switzerland: World Health Organization; 2014.

(2) Damschroder LJ, Aron DC, Keith RE, Kirsh SR, Alexander JA, Lowery JC. Fostering implementation of health services research findings into practice: a consolidated framework for advancing implementation science. Implementation Science: IS. 2009; 4:50-50.

\section{PP102}

Pharmacists' ${ }^{\prime}$ attitudes to anticoagulants with focus on patients ${ }^{\prime}$ education

Simona Dvorackova* ${ }^{1}$, Eliska Kolmanova ${ }^{1}$, Katerina MalaLadova $^{1}$, Tereza Mertova ${ }^{1}$, Jozef Kolar ${ }^{1}$, Josef Maly ${ }^{1}$

${ }^{1}$ Department of Social and Clinical Pharmacy, Charles University, Faculty of Pharmacy, Hradec Králové, Czech Republic

Background and Objective: The objective was to analyze aspects of dispensing, opinions, and attitudes of pharmacists towards direct oral anticoagulants (DOACs) focusing on the perceived benefits and risks of these medicines as well as the level of education provided to patients.

Method: An online questionnaire survey was conducted between March and May 2021 addressing pharmacists from 3 Czech regions located around big cities. Questionnaire comprised 32 items especially focused on frequency of DOACs' dispensing, pharmacist selfconfidence about DOACs, information provided to patients during dispensing of DOACs, and perceptions of differences compared with warfarin. Combination of open, closed and Likert scale answers were employed and analyzed by descriptive statistics. 
Main outcome measures: Confidence, opinions, and attitudes of pharmacists towards DOACs regarding education of anticoagulated patients.

Results: The total of 162 pharmacists (mean age 37.5 years; $91 \%$ women) participated, of whom 139 had an experience with dispensing DOACs in previous year. About $30 \%$ pharmacists dispensed DOACs several times daily and mainly focused on correct dosing and handling the DOACs when giving an information to patients. Only $21.6 \%$ respondents felt insecure during dispensation, but the majority (97.8\%) would appreciate further education regarding DOACs. Pharmacists considered DOACs as safer and equally effective as warfarin in $78.4 \%$ and $41.7 \%$ responds, respectively. More than half respondents $(61.7 \%)$ reported that patients refilling their DOACs in a pharmacy were educated or rather educated from their physician about the therapeutic principles. Pharmacists felt the same responsibility for the patient education as physicians, but they reported limits mostly in lack of time and patients' interest.

Conclusion: Pharmacists are aware of their position in patients' education having quite strong opinion and experience with DOACs, still more effort need to be placed in awareness of patients with these medicines.

\section{PP103}

Development of pharmacy decision rules for cefazolin and ceftriaxone to support clinical prescription validation

\section{Magelien Van den Bulck ${ }^{1}$, Stephanie Wuyts ${ }^{*}$ 1, 2 , Pieter-Jan Cortoos $^{1}$, Pieter Cornu ${ }^{2,3}$}

${ }^{1}$ Pharmacy, University Hospital of Brussels, ${ }^{2}$ Research group Clinical Pharmacology and Clinical Pharmacy, Centre for Pharmaceutical Research, Faculty of Medicine and Pharmacy, Vrije Universiteit Brussel, ${ }^{3}$ Department of Information and Communication Technologies, University Hospital of Brussels, Brussels, Belgium

Background and Objective: Verification of prescription accuracy is a critical step in the medication process as a clinical pharmacist can identify prescribing errors and reduce potential harm to the patient. Approximately $30-50 \%$ of antibiotic prescriptions in hospitals are incorrect or even unnecessary making antibiotics an important group of drugs to target during clinical prescription validation. However, manual prescription review is labor-intensive. We aimed to develop and evaluate decision rules for antibiotics to support prescription validation and standardize pharmacy practice.

Method: Two antibiotics were identified (cefazolin and ceftriaxone), based on a risk assessment, for which decision rules were developed in the form of classification trees. Every end node of the tree was either an 'OK' statement, which suggested appropriate prescribing, or a 'STOP' message, indicating a need for pharmacist review. A random selection was made of all 2019 prescriptions, submitted into the computerized order entry system of the University Hospital of Brussels (UZ Brussel), a Belgian 721-bed tertiary care hospital. These prescriptions were used to validate the decision rules retrospectively by comparing the outcome of the decision rules to a clinical pharmacist's expert opinion. Four performance measures were determined.

Main outcome measures: Specificity, sensitivity, positive and negative predictive value for each decision rule

Results: Both decision trees consisted of six main components: duplicate medication, indication and dosage, dose adjustment to kidney function, pregnancy, breastfeeding and allergy. A total of 585 prescriptions $(50 \%)$ for cefazolin and 170 prescriptions (20\%) for ceftriaxone were validated. The calculated specificity of the decision rules was $99.8 \%$ for cefazolin and $96.9 \%$ for ceftriaxone. Sensitivity was similar, $81.1 \%$ for cefazolin and $81.9 \%$ for ceftriaxone. The positive predictive value for cefazolin and ceftriaxone was $97.3 \%$ and $55.4 \%$, respectively. The negative predictive value was $98.4 \%$ for cefazolin and $99.1 \%$ for ceftriaxone.

Conclusion: The development of pharmacy decision rules for cefazolin and ceftriaxone was considered successful as high performance metrics were calculated. Although these rules could be an additional value in prescription validation, a prospective validation of the decision rules remains required. Additionally, efforts should be made to increase the availability of coded and structured clinical data in the electronic patient record. This would facilitate the integration of the developed content into the hospital information system.

\section{PP104}

Prevalence and risk factors of potentially inappropriate medication use in community-residing older adults: preliminary results from the EUROAGEISM H2020 project

Jovana Brkic $^{*} 1$, Jindra Reissigova ${ }^{2}$, Sofija Sesto ${ }^{3}$, Oznur Altiparmak ${ }^{4}$, Margita Drzaic ${ }^{5}$, Ingrid Kummer ${ }^{1}$, Adriana Magatova $^{1}$, Veera Bobrova ${ }^{6}$, Konstantin Tachkov ${ }^{7}$, Andreas Capiau $^{8,}$, , Akshaya S. Bhagavathula ${ }^{1}$, Deepak K. Bandari ${ }^{1}$, Maria Kamusheva ${ }^{7}$, Daniela Fialova ${ }^{1,10}$

${ }^{1}$ Department of Social and Clinical Pharmacy, Faculty of Pharmacy in Hradec Kralove, Charles University, Hradec Kralove, ${ }^{2}$ Department of Statistical Modelling, Institute of Computer Science, Czech Academy of Sciences, Prague, Czech Republic, ${ }^{3}$ Department of Social Pharmacy and Pharmaceutical Legislation, Faculty of Pharmacy, University of Belgrade, Belgrade, Serbia, ${ }^{4}$ Clinical Pharmacy Department, Faculty of Pharmacy, Marmara University, Istanbul, Turkey, ${ }^{5}$ Faculty of Pharmacy and Biochemistry, University of Zagreb, Zagreb, Croatia, ${ }^{6}$ Institute of Pharmacy, Faculty of Medicine, University of Tartu, Tartu, Estonia, ${ }^{7}$ Department of Social Pharmacy and Pharmacoeconomic, Faculty of Pharmacy, Medical University of Sofia, Sofia, Bulgaria, ${ }^{8}$ Pharmaceutical Care Unit, Faculty of Pharmaceutical Sciences, Ghent University, ${ }^{9}$ Department of Pharmacy, Ghent University Hospital, Ghent, Belgium, ${ }^{10}$ Department of Geriatrics and Gerontology, 1st Faculty of Medicine, Charles University, Prague, Czech Republic

Background and Objective: Detailed knowledge of potentially inappropriate medications (PIMs) and efforts to reduce their unnecessary prescribing are important factors helping to minimise medication-related risks in older adults. In some European countries where clinical pharmacy services are not yet broadly available, PIM use in older patients is expected to be high because of the lack of regular medication reviews. Therefore, the aim of our study was to assess the prevalence and risk factors of PIM use in several European countries.

Method: We conducted a multicenter cross-sectional study in 8 countries in older adults aged $65+$ years visiting community pharmacies (3511 in total). This abstract summarises only preliminary findings on the prevalence and risk factors of PIM use in 5 European countries (Croatia, Czechia, Serbia, Spain and Turkey). Older patients were prospectively assessed using a structured protocol based on the comprehensive geriatric assessment. The protocol included over 300 items on sociodemographic, clinical, functional and medication-related characteristics. Interviews with patients and healthcare professionals, medical records, and clinical assessments were used as the source of data. The prevalence of PIM use was assessed using the EU(7)-PIM list and American Geriatrics Society 2019 Beers Criteria, and stepwise multiple logistic regression was applied to identify potential risk and protective factors. 
Main outcome measures: The prevalence and risk/protective factors of PIM use.

Results: Our preliminary analysis included 2011 older adults; most of them were females $(59.1 \%)$. The prevalence of polypharmacy $(5+$ medications) and polymorbidity (4+ disorders) were $51.4 \%$ and 50.8 $\%$, respectively. The prevalence of PIM use according to both Beers 2019 criteria and EU(7)-PIM list was $61.4 \%$, and it ranged from 37.1 $\%$ in Czechia to $74.4 \%$ in Croatia. The logistic regression model showed the following factors to be significantly associated with PIM use $(\mathrm{p}<0.05)$ : being female 1.30 (1.05-1.61); taking 5+ medications 6.12 (4.94-7.58); being diagnosed with depression 2.37 (1.56-3.61); residing in Czechia $0.30(0.21-0.41)$ and Turkey $0.45(0.33-0.63)$ compared to Croatia.

Conclusion: These preliminary findings confirm that PIM use in geriatric patients is highly prevalent in some European countries, particularly in Spain, Serbia and Croatia (with the prevalence above $60 \%$ ). Furthermore, polypharmacy and depression were significantly correlated with PIM prescribing in older adults. Therefore, optimisation of drug therapy by clinical pharmacists should primarily focus on these cohorts of older adults.

References: Grants: EuroAgeism H2020 MSCF-ITN-764632, Inomed NO.CZ.02.1.01/0.0/0.0/18_069/0010046, Progress Q42Faculty of Pharmacy, Charles University, START/MED/ 093CZ.02.2.69/0.0/0.0/19_073/0016935, SVV 260551 and ICARE4OLD H2020 -965341.

\section{PP105}

The impact of COVID-19 on smoking behaviours and support for smoke-free zones in Saudi Arabia

\section{Mansour Tobaiqy $^{*}{ }^{1}$, Andrew MacLure ${ }^{2}$, Dennis Thomas ${ }^{3}$, Katie MacLure $^{4}$}

${ }^{1}$ Pharmacology, College of Medicine, University of Jeddah, Jeddah, Saudi Arabia, ${ }^{2}$ Independent Researcher, Aberdeen, United Kingdom, ${ }^{3}$ University of Newcastle, NSW, Australia, ${ }^{4}$ Independent Research Consultant, Aberdeen, United Kingdom

Background and Objective: Globally, the World Health Organization (WHO) engages with countries to adopt policies and strategies which strive to encourage and support people to quit smoking. However, to date, only 23 countries were on track before the global Covid-19 pandemic to meet the target. Undoubtedly, cigarette smoking is associated with increased risk for the development of cancer and cardiovascular diseases, both of which were the leading causes of death in recent decades. This research focuses on the impact of Covid-19 on smoking and smoking cessation behaviours and support for smoke-free zones in Jeddah, Saudi Arabia.

Design: A pre-tested structured survey was distributed by email in October-November 2020 to students and staff at the University of Jeddah. Responses were analysed using descriptive statistics with summative content analysis of open text.

Results: Participants providing open text comments $(n=374 / 666$; $56.4 \%)$ were non-smokers $(n=293 ; 78.3 \%)$, former smokers $(n=26$; $7.0 \%)$ and current smokers $(\mathrm{n}=55 ; 14.7 \%)$. Some had household members $(n=220 ; 58.8 \%)$ and friends who smoke $(n=198 ; 52.9 \%)$ plus daily exposure to secondhand smoke at home $(n=125 ; 33.4 \%)$. There was an awareness during Covid-19 of: smoking inside cafes/ restaurants and other indoor and outdoor public places; exposure to warnings in the media both against and promoting smoking; widespread support for smoke-free zones. Smokers plans for accessing smoking cessation support are inconsistent with retrospective reports. Many express positivity highlighting reductions in smoking but there were also negative reports of increased smoking.
Conclusion: The Covid-19 pandemic has affected every aspect of society worldwide. People have been at home more with restricted freedom of movement and limitations on social liberty. These individual accounts can help to focus evidence-based smoking prevention and cessation programmes during and post-Covid-19.

\section{PP106}

Establish a pharmaceutical emergency management system in China: lessons learned from the COVID-19

Mimi Tang* ${ }^{1}$, Ting Liu ${ }^{1}$, Tingyu Zhao", Shao Liu', Shusen Sun ${ }^{2}$, Zhicheng Gong ${ }^{1}$

${ }^{1}$ Department of Pharmacy, Xiangya Hospital, Central South University, Changsha, China, ${ }^{2}$ Department of Pharmacy Practice, College of Pharmacy and Health Sciences, Western New England University, Springfield, United States

Background and Objective: Emergency management is an important embodiment of a country's comprehensive strength, and a safety guarantee for building a harmonious society. However little research has been focused on developing pharmacy emergency management. Pharmacists are indispensable in emergency pharmaceutical treatment. The COVID-19 that rapidly spreads globally exposed the shortcomings of emergency pharmaceutical management system in China. Therefore, it is significant to build the guarantee platform of information, capacity and funding in emergencies to optimize the pharmacy emergency management system, and to further ensure the effectiveness of pharmacists in emergency control, which will consequently improve the quality and efficiency of medical rescue.

Design: Future efforts should be focus on the following issues.

1) Evaluation indicator construction: Preliminarily formulate the framework of emergency rescue ability evaluation indicator system of pharmacists by literature analysis and free interview. One component of the indicator system is basic skills such as management, dispensing, quality control of medicines, pharmaceutical service, information arrangement and communication ability; while the other component is emergency capacity, referring to personal protection, the ability to adapt to new position and environment, and psychological rescue abilities, such as identification, prevention and intervention of psychological problems. Evaluation indicators will be further determined after expert consultation and analytical hierarchy process, and the weight values of indicators will be determined before the emergency rescue ability evaluation indicator system of pharmacists could be constructed.

2) Course design for the training of emergency pharmaceutical administration rescue ability: learning objectives and course contents of the emergency administration of pharmacy courses should be set up. Teaching/learning strategies, as well as feedback/evaluation strategies targeting learning objectives should be selected by means of course development technology. Furthermore, the emergency administration of pharmacy courses elements, including laws and regulations, emergency pharmaceutical administration system, counter-plan system according to the classification of disaster, and monitoring and forecasting system, should be integrated, so as to form a core course proposal of emergency pharmaceutical rescue training. Results: Established pharmaceutical emergency management courses include medicine management and storage, use and management of disinfector, selection of medicine, drug clinical trial management. Pharmacists can enhance their ability to deal with public health events, and a contingency team made up of clinical pharmacists with great professional competence can be built.

Conclusion: It's important to assess the emergency ability of pharmacists, and construct systematic and specific emergency 
pharmaceutical management courses to train pharmacists for public emergency events, and improve the efficiency and quality of the pharmacists in rescue team.

\section{PP107}

What should be the role of the pharmacist in the treatment of depression?

\section{Zekiye K. Yılmaz ${ }^{*}$, İrem T. Aslanbay ${ }^{1}$ \\ ${ }^{1}$ Clinical Pharmacy, Acıbadem Mehmet Ali Aydınlar University, Faculty of Pharmacy, Istanbul, Turkey}

Background and Objective: Depression, is the most common psychiatric disorders which affects more than 300 million people worldwide (1). Untreated depression can negatively affect the general health of patients and cause unexpected early deaths. It is important to know the depressive symptoms and antidepressant use behaviors of university students, as they constitute an important part of the general depressive population. Comprehensive screening tools for diagnosing depression can facilitate early diagnosis (2). As the most reachable healthcare professionals, pharmacists can act a prominent role in helping identify individuals at risk (3). The aim of this study is to determine the prevalence of depression, use of antidepressants and the frequency of untreated depression in university students.

Method: A prospective pilot observational study was carried out on university students all around the Turkey. It was a short-course design from 4 to 11 June 2021. Patient health questionnaire-9 and additional questions was applied to participants via Google forms by sharing the survey link.

Main outcome measures: Sociodemographic characteristics of students. Whether or not the student have been diagnosed with depression, used antidepressant or had patient education about medicine usage. Assessment of patient health questionnaire-9.

Results: A total of 73 university students were participated in this study with a mean age of $21.99 \pm 1.94$ years constituting $54(74 \%)$ females. $26(35.6 \%)$ of the students study at a public university. 54 (74\%) of the participants were living with their families. $15(20.5 \%)$ of the participants has diagnosed with depression at least once in a lifetime and $20(27.4 \%)$ has used antidepressants at least once in a lifetime. $15(20.5 \%)$ of the participants were using medicine(s) regularly and only $10(13.7 \%)$ of the participants were being given patient education by the pharmacist. It was detected that $47(64.39 \%)$ of the university students have moderate to severe depressive symptoms and $36(49.32 \%)$ of these students were female. It was detected that 35 $(47.95 \%)$ of the students who have moderate to severe depressive symptoms have never used antidepressant in their life. The most commonly used antidepressants by the participants were paroxetine 9 $(12.3 \%)$, sertraline $5(6.8 \%)$ and escitalopram $3(4.1 \%) .10(13.7 \%)$ of the participants stated that they suffered from the side effects of the medicine they used.

Conclusion: The results of this study has shown that 35 (47.95\%) of the students who have moderate to severe depressive symptoms were not having treatment. As untreated depression can cause unexpected early deaths and negatively affect the general health, it is critical for these group of individuals to be referred to the physician for further assessment. As the most accessible healthcare professionals, pharmacists can play an important role in helping identify individuals at risk. In addition, they should take a more active role in increasing the compliance of patients under drug therapy and providing patient education.
References:1. Cacheda F, Fernandez D, et al.Early detection of depression: Social network analysis and random forest techniques. J Med Internet Res. 2019;21(6):e12554.

2. O'Reilly CL, Wong E, Chen TF. A feasibility study of community pharmacists performing depression screening services. Res Social Adm Pharm. 2015;11(3):364-81.

3. Kondova A, Todorova A, et al. Screening and risk assessment for depression in community pharmacy-pilot study. J of IMAB. 2018;24(1):1928-1931.

\section{PP108}

A scoping review of the methods and processes used by regulatory bodies to determine pharmacists' readiness to practice

\section{Eimear Ni Sheachnasaigh $^{*}$, Cristin Ryan ${ }^{1}$, Cathal Cadogan ${ }^{1}$ \\ ${ }^{1}$ Practice of Pharmacy, Trinity College Dublin, The University of Dublin, Dublin, Ireland}

Background and Objective: There is an expectation from government, regulatory bodies, patients, the public, and other healthcare professions that pharmacists are competent professionals who can practice independently. Regulation of the profession requires pharmacy graduates to register with a recognised regulatory body before being considered 'ready to practice' independently. This scoping review examined the methods and processes used by national regulatory bodies to determine pharmacists' readiness to practice.

Method: This scoping review followed the Joanna Briggs Institute guidelines ${ }^{1}$. Searches were conducted using three electronic databases (EMBASE, CINAHL and Scopus). Websites for national pharmacy regulators identified during the searches were also reviewed for grey literature. Data were selected based on inclusion and exclusion criteria pre-specified in the protocol. Sources were screened by three reviewers, independently. Any disagreements were resolved by consensus discussion. Authors of primary sources and regulatory bodies were contacted for further information, where necessary. A narrative synthesis was conducted.

Main outcome measures: Overview of methods and processes used by national regulatory bodies to determine pharmacists' readiness to practice.

Results: 1,959 articles were screened and 15 studies included across 11 countries for which national regulatory body websites were reviewed for grey literature. None of the identified sources provided a definition of the term 'ready to practice'. Ten countries were identified as holding a registration examination with varying formats and curricula. Identified assessment methods included written and oral knowledge-based exams, written competency-based assessments, Objective Structured Clinical Examinations (OSCEs) and combination of these, with written format being the most common $(n=8)$. In all but one country (Ireland), the regulator was responsible for delivery of the exam. In the majority of countries $(n=7)$, the exam was mapped to a pre-defined set of competencies with only four countries explaining how these competencies were developed.

Conclusion: There is a paucity of research and publicly available information on the methods and processes used by national regulators to determine pharmacists' readiness to practice. There is no pharmacy definition of being 'ready to practice'. Assessment methods vary widely and currently, no gold standard is apparent.

References: Peters et al. JBI Evid Synth. 2020;18(10):2119-26 


\section{PP109}

GPS' views/experiences of pharmacist services in general practice: a qualitative evidence synthesis

Eoin Hurley $^{*}{ }^{1}$, Laura Gleeson ${ }^{2}$, Stephen Byrne ${ }^{1}$, Elaine Walsh ${ }^{3}$, Tony Foley ${ }^{3}$, Kieran Dalton ${ }^{1}$

${ }^{1}$ School of Pharmacy, University College Cork, Cork , ${ }^{2}$ School of Pharmacy and Biomolecular Sciences, Royal College of Surgeons in Ireland, Dublin, ${ }^{3}$ Department of General Practice, University College Cork, Cork, Ireland

Background and Objective: Pharmacist services in general practice are expanding worldwide, with evidence to show that pharmacists in this setting reduce the number of patients' appointments with their general practitioner (GP), improve medication management, and reduce medication costs. Yet, little is known globally about GPs' views of the role of the pharmacist in this context.

Therefore, the primary objective of this study was to synthesise and appraise the qualitative research evidence on GPs' views and experiences of pharmacist services in general practice, with an assessment of confidence in the review findings.

Method: Eight electronic databases were systematically searched from inception to April $9^{\text {th }} 2021$ for qualitative studies that reported the views/experiences of GPs regarding pharmacist services in general practice. The quality of included studies was appraised using the Critical Appraisal Skills Programme (CASP) checklist for qualitative studies. Data from included studies were analysed using thematic synthesis. The GRADE Confidence in the Evidence from Reviews of Qualitative research (CERQual) approach was used to assess confidence in the review findings. The review protocol was registered on the International Prospective Register of Systematic Reviews (PROSPERO) database as CRD42021224508.

Main outcome measures: Views and experiences of GPs regarding pharmacist services in general practice.

Results: Nineteen studies were included, which captured the views of 159 GPs across eight countries: Australia $(n=6)$, Canada $(n=3)$, England $(\mathrm{n}=3)$, the United States $(\mathrm{n}=3)$, Iceland $(\mathrm{n}=1)$, Ireland $(\mathrm{n}=$ $1)$, Malaysia $(n=1)$, and Scotland/England $(n=1)$. A conceptual model was created that describes the factors to be considered in the development or optimisation of pharmacist services in general practice based on the views and experiences of GPs. This model consists of four overarching analytical themes, each encompassing a number of descriptive themes, which were generated from the coded data: (1) optimal environmental/contextual conditions to host a pharmacist (including pre-implementation planning, logistics, and financial considerations), (2) the ideal pharmacist characteristics (including activities/role, desirable skills, and desirable/undesirable qualities), (3) complex stakeholder relationships with the pharmacist (including encroachment), and (4) benefits of an effective pharmacist (including those to the GP, the practice, and to the patient). Over $60 \%$ of findings were graded as moderate or high confidence using the CERQual approach.

Conclusion: The conceptual model and themes generated in this review may form a useful framework for GPs, pharmacists, researchers, and policymakers not only in designing future interventions and qualitative research studies, but also in optimising the process of integrating a pharmacist into general practice and current pharmacist services in general practice.

\section{PP110}

Evaluation of pharmaceutical care services in the Middle East countries: a review of studies of 2013-2020

\section{HEBAH M. A. SALLOM* ${ }^{*}$, ABDIKARIM ABDI², BILGEN BAŞGUST $^{3}$}

${ }^{1}$ Department of Clinical Pharmacy, Faculty of Pharmacy, Near East University, Nicosia, North Cyprus, ${ }^{2}$ Department of Clinical Pharmacy, Faculty of Pharmacy, Yeditepe University, Istanbul, ${ }^{3}$ Department of Pharmacology, Faculty of Pharmacy, Baskent University, Ankara, Turkey

Background and Objective: Over the past decades pharmaceutical care services (PCs) continued to evolve globally with increasing emphasis on patients and proven effectiveness in variety of settings. Evidence supporting this practice is mostly from the US, yet the evaluation and adoption of PCs varies across different regions of the world.

Objective: To evaluate and determine the effectiveness of pharmaceutical care services in various pharmaceutical aspects within 17 countries from the Middle- East countries.

Design: Arkesy and O'Malley framework was used to conduct a scoping review. This review was undertaken in 6 databases: PubMed/ Medline, Scopus, Cochrane Library, Springer Link, Clinical Trail and Web of Science. The quality of studies was evaluated using Van Tulder Scale for Randomized studies and Down and Black checklists for Non-Randomized studies. General descriptive and numerical analysis regarding selected studies and thematic analysis for included studies which created 4 main themes: Scope of applicable PCs, Pharmaceutical implementers, studies outcomes and quality.

Results: This study identified 431,753 citations, and after a review of 271 full-text papers, there were 132 eligible articles which were involved in the study. Study design varied, with 43 (32.6\%) RCTs and $89(67.4 \%)$ n-RCTs. Thirty-five $(26.5 \%)$ of included studies were published in 2020. Majority of the studies had been conducted in Jordan, KSA followed by Turkey, respectively (25.8\%, $16.7 \%$, $11.4 \%)$. Thirty-seven (19.7\%) studies were aimed at resolving DRPs, $27(14.4 \%)$ in improving QoL and 23(12.2\%) for increasing medication adherence. Also, the results emphasized that mean scores of the activities provided to patients each year increased significantly.

Conclusion: Studies in the Middle East continue to provide evidence supporting the positive impact of pharmaceutical care services on both hard and soft outcomes measured in most studies. Yet, rigorous evaluation of economic impact of introduced services and their sustainability is must.

\section{PP111}

Assessing the completeness and exhaustiveness of the national cancer registry in a cohort of advanced breast cancer women

Inês Teodoro $^{*}$ 1, Fábio C. Borges², Ana C. Miranda ${ }^{2}$, Filipa A. Costa $^{1,2}$

${ }^{1}$ Centro de Investigação Interdisciplinar Egas Moniz (CiiEM), Instituto Universitário Egas Moniz (IUEM), Almada, ${ }^{2}$ Registo Oncológico Nacional (RON), Instituto Português de Oncologia de Lisboa Francisco Gentil (IPOLFG), Lisboa, Portugal

Background and Objective: Advanced breast cancer (ABC) is the leading cause of mortality by cancer among women, therefore it is important to understand the outcome of the approved therapies in real-world (1). The National Cancer Registry database (NCRDB) is a relevant source for carrying out these studies, however, it is necessary 
to ensure the quality and exhaustiveness of the recorded information (2). This retrospective cohort study, submitted and approved to the ethic commission of Portuguese Oncology Institute of Lisbon (IPOL), aimed to explore the exhaustiveness of demographic and clinical variables in a cohort of females $>18$ years, with histologically confirmed diagnosis of ABC (stages IIIC and IV) that underwent therapy with fulvestrant between May 2017 and March 2019, at the IPOL.

Method: Using patients, included in hospital pharmacy database, who started therapy with fulvestrant in this period, anonymized data were exported from NCRDB, taking all other criteria into account, and subsequently analysed using Microsoft Excel, version 16.45. In a sample including 109 cases, a descriptive analysis of the exhaustiveness of demographic and clinical variables was performed, considering three levels for exhaustiveness: high (missing values $<$ $1 \%$ ), medium (missing values $1-15 \%$ ) and reduced (missing values $>15 \%$ ) (3).

Main outcome measures: Data will be updated until the exhaustiveness of every variable is ensured.

Results: A high level of exhaustiveness was observed in age, residence, date of diagnosis, topography, morphology and treatment. With a medium level there were cancer differentiation and oestrogen receptors, and reduced levels were observed in progesterone receptors, HER2, Ki67 and focality.

Conclusion: This analysis allowed the identification of variables with lower levels of exhaustiveness, which will require more rigorous update at IPOL. The same analysis, in a similar cohort (aromatase inhibitors instead of fulvestrant) will be performed and data updated until exhaustiveness in both cohorts is ensured. The final goal is to compare the effectiveness of these therapies in real world with trial efficacy outcomes, for example in overall and progression free survival.

References: 1. Cardoso, F., Paluch-Shimon, S., Senkus, E., Curigliano, G.,Aapro, M. S., Andre, F., ... Winer, E. P. (2020). 5th ESOESMO international consensus guidelines for advanced breast cancer (ABC 5) †. Annals of Oncology, 0(0). https://doi.org/10.1016/ j.annonc.2020.09.010

2. Donnelly, C., Cairnduff, V., Chen, J. J., Kearney, T., Fitzpatrick, D., Fox, C., \& Gavin, A. (2017). The completeness and timeliness of cancer registration and the implications for measuring cancer burden. Cancer Epidemiology, 49, 101-107. https://doi.org/10.1016/ j.canep.2017.05.007

3. Verdasca, J., Costa, F. A., Ramos, C., Murteira, R., \& Miranda, A. (2019). The South Region Cancer Registry: an evaluation of its exhaustiveness in a cohort of lung cancer patients. Thoracic Cancer, 10(2), 330-334. https://doi.org/10.1111/1759-7714.12915

Publisher's Note Springer Nature remains neutral with regard to jurisdictional claims in published maps and institutional affiliations. 